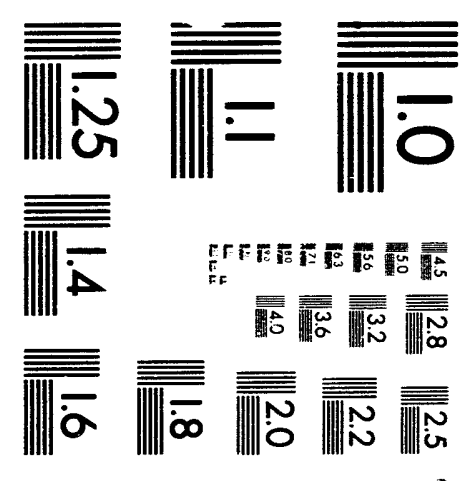



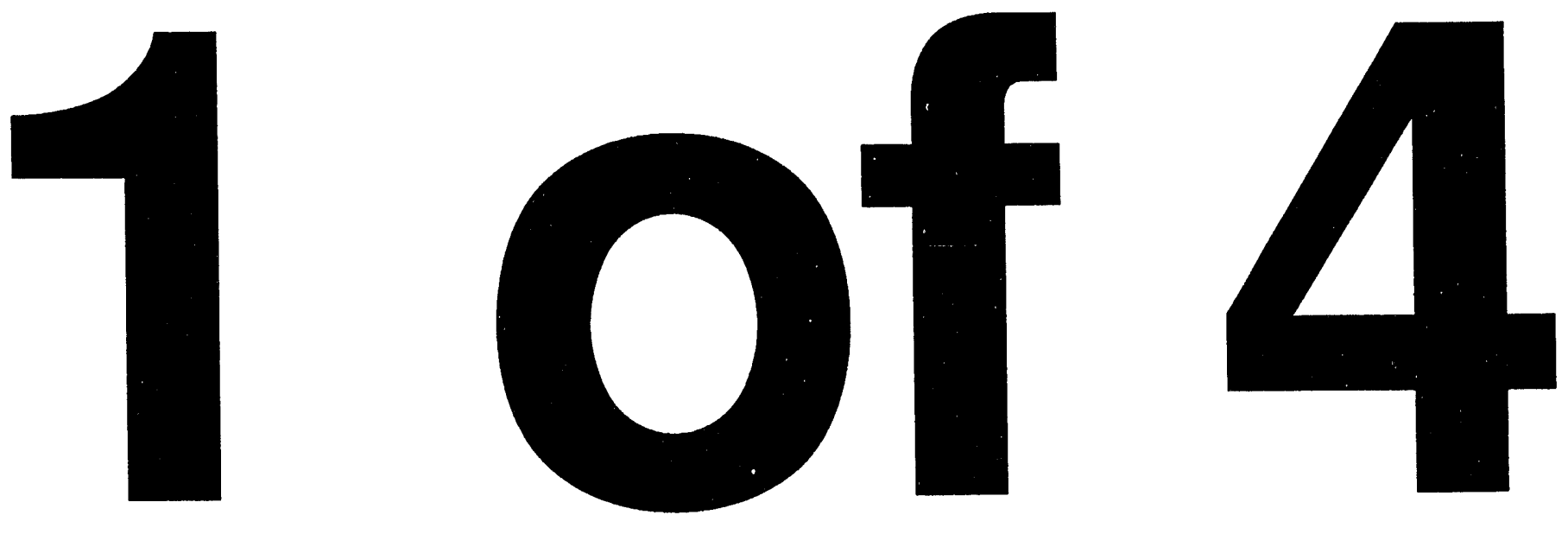


\title{
STUDIES OF HEAVY-ION REACTIONS AND TRANSURANIC NUCLEI
}

\author{
Progress Report \\ September 1, 1992 - August 31, 1993
}

W. U. Schröder

University of Rochester

Rochester, New York 14627

${ }^{209} \mathrm{Bi}+{ }^{136} \mathrm{Xe}$ at $\mathrm{E} / \mathrm{A}=28 \mathrm{MeV}$

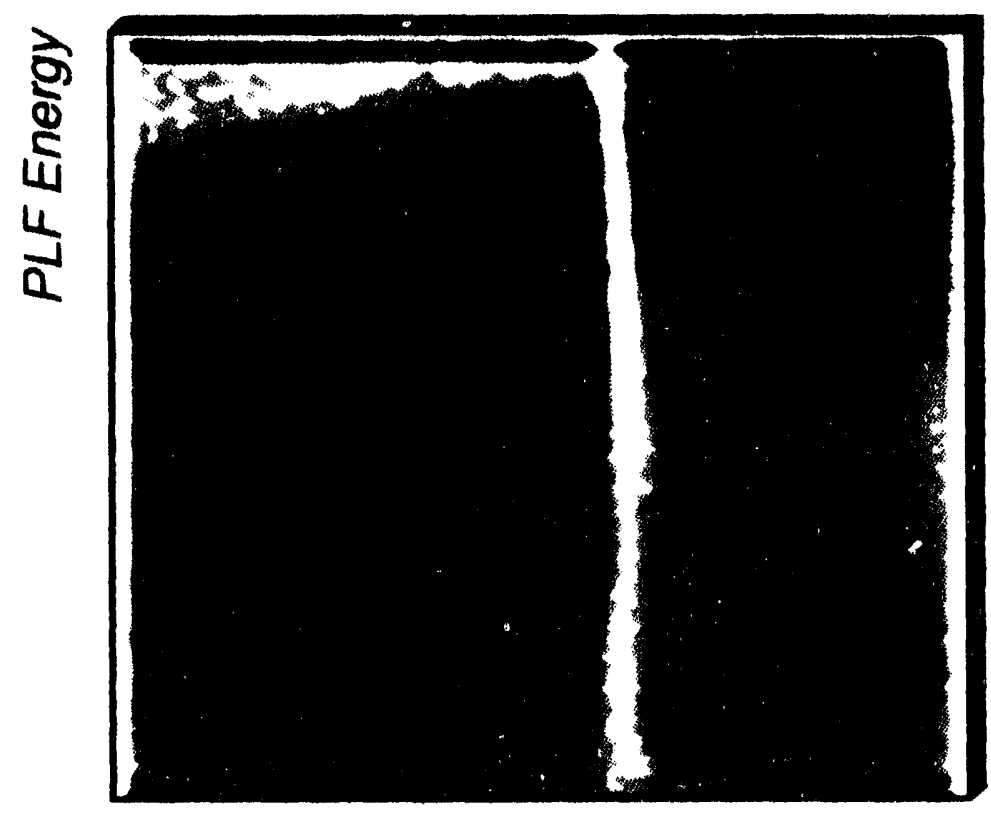

PLF Deflection Angle

August 1993

Prepared for

THE U. S. DEPARTMENT OF ENERGY AGREEMENT NO. DE-FG02-88ER40414 


\title{
STUDIES OF HEAVY-ION REACTIONS AND TRANSURANIC NUCLEI
}

\author{
Progress Report for the Period \\ September 1, 1992 - August 31, 1993 \\ University of Rochester, Department of Chemistry \\ Rochester, New York 14627
}

\author{
August 1993 \\ Prepared for \\ THE UNITED STATES DEPARTMENT OF ENERGY \\ UNDER GRANT NO. DE-FG02-88ER40414
}

\section{NOTICE}

This report was prepared as an account of work sponsored by the United States governinent. Netther the United States nor the United States Department of Energy, nor any of their employees, nor any of their contractors, subcontractors, or their employees, makes any warranty, express or implied, or assumes any legal liability or responsibility for the accuracy, completeness, or usefulness of any information, apparatus, product, or process disclosed or represents that its use would not infringe privately owned rights.

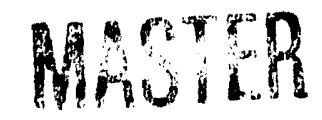




\section{TABLE OF CONTENTS}

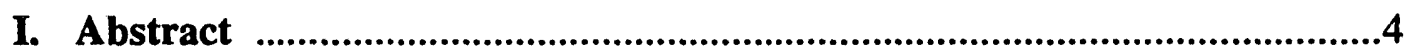

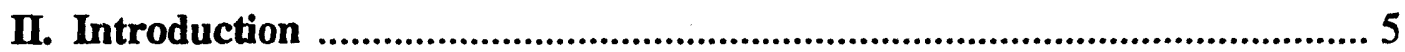

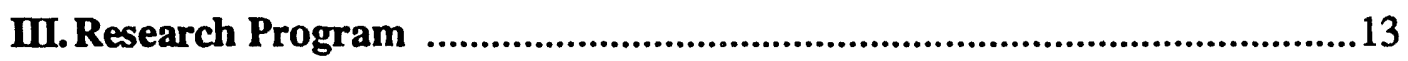

A. The Cold-Fusion Saga ……...................................................................14

B. Decay Patterns of Dysprosium Nuclei Produced in ${ }^{32} \mathrm{~S}+118,124 \mathrm{Sn}$ Fusion Reactions .20

C. Unexpected Features of Reactions Between Very Heavy Ions at Intermediate Bombarding Energies

D. Correlations Between Neutrons and Charged Products from the Dissipative Reaction ${ }^{197} \mathrm{Au}+{ }^{208} \mathrm{~Pb}$ at E/A $=29 \mathrm{MeV}$ .51

E. Dissipative Dynamics of Projectile-Like Fragment Production in the Reaction ${ }^{209} \mathrm{Bi}+{ }^{136} \mathrm{Xe}$ at $\mathrm{E} / \mathrm{A}=28.2 \mathrm{MeV}$

F. Dynamical Production of Intermediate-Mass Fragments in Peripheral ${ }^{209} \mathrm{Bi}+{ }^{136} \mathrm{Xe}$ Collisions at $\mathrm{E}_{\mathrm{lab}} / \mathrm{A}=28.2 \mathrm{MeV}$

G. The Rochester 960-Liter Neutron Multiplicity Meter 141

H. A Simple Pulse Processing Concept for a Low-Cost Pulse-Shape-Based Particle Identification

I. A One-Transistor Preamplifier for PMT Anode Signals 185

J. A Five-Channel Multistop TDC/Event Handler for the SuperBall Neutron Multiplicity Meter

K. Construction of the SuperBall - a 16,000-Liter Neutron Detector for Calorimetric Studies of Intermediate-Energy Heavy-Ion Reactions 201

L. A Computer Code for Light Detection Efficiency Calculations for Photomultipliers of a Neutron Detector

M. Evaluation of Gd-Loaded Liquid Scintillators for the SuperBall Neutron Calorimeter

N. Measurement of the Interaction of Cosmic-Ray $\mu^{-}$with a Muon Telescope 


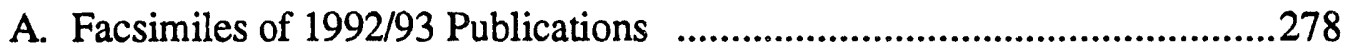

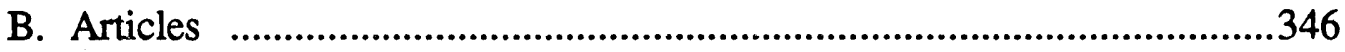

C. Invited Lectures

D. Contributed Papers at Professional Meetings ..........................................350

E. Presentations before NSF and NSAC Committees …..............................351

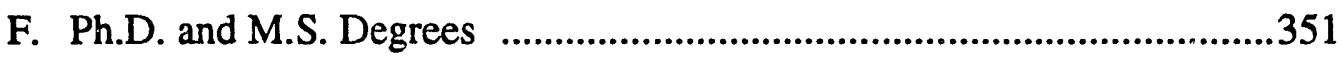

G. Professional Activities ..........................................................................352

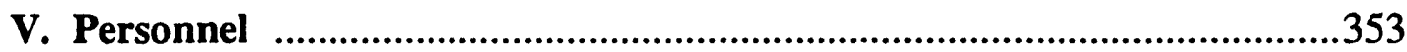




\section{Abstract}

New developments around the "cold fusion" phenomenon are reviewed critically. The thermodynamics of the decay of Dy composite nuclei was studied in measurements of evaporated neutrons and light particles, confirming earlier conclusions with respect to the absence of significant entrance-channel effects in compound-nucleus reactions. A survey is given over the most significant findings made in exclusive measurements for the veryheavy-ion reactions ${ }^{197} \mathrm{Au}+{ }^{208} \mathrm{~Pb}$ and ${ }^{209} \mathrm{Bi}+{ }^{136} \mathrm{Xe}$ at $\mathrm{E} / \mathrm{A} \approx 30 \mathrm{MeV}$, including the first experiment ever to measure neutrons and charged particles with full angular coverage. The exclusive measurement of neutrons from the reaction ${ }^{197} \mathrm{Au}+{ }^{208} \mathrm{~Pb}$ revealed, essentially for all impact parameters, the prevalence of a dominantly binary reaction mechanism, followed by the decay of the primary fragments. Relatively small multiplicities were observed for non-equilibrium neutron emission. Correlations between observables characteristic of hinary dissipative collisions are recaptured at the present intermediate bombarding energies. However, the reaction mechanism appears to be less efficient than at lower bombarding energies in dissipating the available kinetic energy. For the ${ }^{209} \mathrm{Bi}+{ }^{136} \mathrm{Xe}$ reaction, it was possible to reconstruct the primary fragment atomic number distributions, seen to agree with the predictions of a one-body transport mechanism. For this reaction, the projectile-like fragments, exhausting the total reaction cross section, show a double-branched angle-energy correlation reminiscent of the orbiting or rainbow-scattering picture. Surprisingly, intermediate-mass fragments are found to be emitted in peripheral reactions with angle-energy patterns consistent with multiple rupture of the intermediate dinuclear system. For more central collisions, such fragments are observed to be emitted sequentially from the excited primary fragments.

Significant progress is reported in technological developments. The performance of the present Rochester neutron multiplicity meter is described. Several electronics modules have been developed in anticipation of future experiments, including a very low-cost particle identifier, a very effective one-transistor preamplifier, and a complex multichannel time-to-digital converter/event handler for the new SuperBall neutron calorimeter. A status report is given on the construction of this detector. A computer code for the simulation of the light collection process in the SuperBall tank elements is described. Test measurements of various liquid scintillators for the calorimeter are discussed. 


\section{Introduction}

This report describes the research activities of the University of Rochester Nuclear Chemistry group over the period from September 1992 to August 1993. The research program is supported by the United States Department of Energy under Grant DE-FG0288ER40414. Experimental work was performed by the group at several national and foreign laboratories, including the University of Rochester Nuclear Structure Research Laboratory, the National Superconducting Cyclotron Laboratory at Michigan State University, and the French heavy-ion laboratory GANIL. Projects have been pursued jointly with groups of nuclear scientists from Washington University at St. Louis, from Michigan State University at East Lansing, from Ball State University at Muncie, from Indiana University at Bloomington, from the French Laboratories GANIL and LPC at Caen, from the CRN Strasbourg, from the IPN Orsay, from the Polish Institute of Experimental Physics and the Heavy-Ion Laboratory in Warsaw, as well as from the German Hahn-Meitner-Institut Berlin.

The work described below includes experimental measurements of heavy-ion-induced reactions both, at energies close to the interaction barrier and at the lower boundary of the Fermi energy regime. It also includes theoretical modeling and simulation calculations. In addition, the group has developed several new electronic devices and performed design and pilot studies for the SuperBall, an advanced segmented $4 \pi$ neutron calorimeter for heavy-ion reaction studies, whose mechanical construction is now almost completed. During this past grant year, much effort went into the design of the SuperBall and the modeling of the detector response. Coordination of construction and acquisition of its components and accessories proved also quite time consuming. The very valuable assistance received in this endeavor from the MSU/NSCL technical staff is greatly appreciated. Most of the discussion in this scientific report is devoted to the new experimental findings made for the two very-heavy-ion reactions ${ }^{197} \mathrm{Au}+208 \mathrm{~Pb}$ and ${ }^{209} \mathrm{Bi}+{ }^{136} \mathrm{Xe}$, belonging to the heaviest systems studied thus far at intermediate bombarding energies $(E / A=30 \mathrm{MeV})$. It now appears very likely that studies of reactions induced by very heavy ions provide a new access to the mechanisms operating at intermediate bombarding energies, the possibly non-adiabatic dynamics of nuclear collective motion, and the prospects of creating nuclear matter at critical temperatures.

Although our group has never been actively involved in studies of "cold fusion", claimed to have been induced in electro-chemical experiments, one of us (JRH) has 
played a definitive role in exarining and discussing such claims critically. In spite of almost unanimous reservation by the nuclear science community, claims that "cold fusion" in a bench-top electro-chemical cell is a nuclear process producing excess power of the order of Watts are still being upheld. Excerpts of a new critical publication (by JRH) reporting on the status of experimental and theoretical cold-fusion studies are included in this report.

The fusion-evaporation process, as a model of the formation and the decay of equilibrated nuclear systems, is one of the pillars of nuclear reaction theory and of immense practical significance. A series of novel experiments was continued, investigating disturbing anomalies reported in the literature for the behavior of composite systems in the rare-earth region. These experiments included a characterization of the thermodynamic state of an excited composite nucleus by an exclusive measurement of several moments of the associated-neutron multiplicity distribution, employing the Rochester 9600- $l$ neutron multiplicity meter (NMM). Following the discovery that differences in the level densities of Gd composite nuclei, rather than a superdeformation of their shapes during the fusion process, is responsible for the reported anomalies, it was necessary to confirm the trends observed thus far only with one set of isotopes. The new measurements of the decay patterns of Dy nuclei, produced in ${ }^{32} \mathrm{~S}+{ }^{118,124} \mathrm{Sn}$ fusion reactions, is reported in the present report. In support of earlier conclusions, it is found that a memory of entrance-channel conditions does not survive the formation stage of Dy compound systems.

Exclusive measurements of neutrons, light charged particles, intermediate-mass fragments and massive reaction products have been performed for the two heavy-ion systems ${ }^{197} \mathrm{Au}+{ }^{208} \mathrm{~Pb}$ and ${ }^{209} \mathrm{Bi}+{ }^{136} \mathrm{Xe}$, both at the lower boundary of the Fermi energy domain. The experiments provided unexpected, but compelling evidence for very simple reaction dynamics dominated by collective degrees of freedom. In these experiments on very heavy, neutron-rich systems, the neutron multiplicity was found to provide a sensitive selection of reaction phenomenology. For future investigations of intermediate-energy heavy-ion reaction mechanisms, this multiplicity observable could play a role as significant and elucidating as that of the total kinetic-energy loss in studies of low-energy fission and heavy-ion collisions. In a survey of the most important findings of this study, attention is called to the fact that one may observe new, nonadiabatic effects in energetic nuclear collisions, which could cast serious doubt on the 
validity of adiabaticity or mean-field assumptions made usually in heavy-ion reaction theory.

The analysis of correlations between charged products and neutrons produced in the reaction ${ }^{197} \mathrm{Au}+{ }^{208} \mathrm{~Pb}$ at $\mathrm{E} / \mathrm{A}=29 \mathrm{MeV}$ was finalized, yielding a consistent picture of the underlying reaction mechanism. In this elaborate experiment, the time of flight was measured for neutrons in coincidence with individual or pairs of correlated charged products emitted at forward angles. A kinematical-coincidence measurement provided a calibration for the neutron-multiplicity observable in terms of dissipated energy. Consistently for all types of charged-product event triggers, the invariant velocity distributions of coincident neutrons were found to be essentially bimodal, indicative of a binary primary collision, followed by the sequential decay of the hot reaction primaries. A weak non-equilibrium neutron component was also observed, which, at the present energy, does not play an important role in the balance of reaction energy or collision kinematics. Projectile-like fragments, measured at forward angles, show distributions consistent with predictions of the one-body multi-nucleon transport mechanism. However, the competition between fission and particle evaporation alters the primary distributions significantly and in a characteristic, almost deceptive fashion. Another important observation pertains to the inability of the reaction to dissipate more than about $75 \%$ of the total energy available in the entrance channel, for any class of reaction events selected in this work.

In the second extensive experiment, studying the reaction ${ }^{209} \mathrm{Bi}+{ }^{136} \mathrm{Xe}$ at $\mathrm{E} / \mathrm{A}=28$ $\mathrm{MeV}$, for the first time a simultaneous $4 \pi$ measurement of neutrons and charged products was achieved. In this experiment, cross sections for the production of massive fragments were measured relative to elastic scattering. It was found that essentially the entire cross section ( $\pm 10 \%$ uncertainty) is associated with binary dissipative collisions, leading to massive projectile-like (PLF) and target-like (TLF) fragments. However, the primary fragments can undergo significant alterations through sequential decay, for the most dissipative collisions. The efficient detector setup allowed a reconstruction of the PLF atomic-number distribution. Correlations between the neutron multiplicity, measured over $4 \pi$, and PLF kinetic energy, deflection angle, and atomic-number distributions reveal features very similar to those found for various systems at lower bombarding energies. In particular, the spectacularly clear correlation between fragment energy and detection angle (Wilczyñski Diagram) demonstrates a collective phenomenon much like the orbiting process known for asymmetric interaction systems at lower 
bombarding energies. Many of the observed correlations between experimental observables are qualitatively as expected from transport processes described by the wellknown multi-nucleon exchange model.

Of particular importance for an understanding of the reaction mechanism operating in intermediate-energy heavy-ion reactions is the observation of new nuclear decay modes, such as the emission of intermediate-mass fragments (IMF). In the reaction ${ }^{209} \mathrm{Bi}+{ }^{136} \mathrm{Xe}$ under study, the average IMF multiplicity $\left\langle\mathrm{m}_{\mathrm{IMF}}\right\rangle$ is relatively small, not exceeding values of 1-2 per event. However, this average multiplicity is seen to increase with increasing energy dissipation, as measured either by neutron multiplicity or the atomic number of the PLF remnant. For events with the highest neutron multiplicity, up to 14 IMF's have been observed in the ${ }^{209} \mathrm{Bi}+{ }^{136} \mathrm{Xe}$ reaction, with emission patterns suggesting dominantly sequential evaporation from the primary massive fragments. Such IMF's are frequently observed in relatively central intermediate-energy heavy-ion reactions, although the production mechanisms are rarely as evident as in the present study. Another and perhaps even more interesting IMF emission mode was observed for peripheral ${ }^{209} \mathrm{Bi}+{ }^{136} \mathrm{Xe}$ collisions, with IMF emission patterns that are clearly inconsistent with sequential evaporation. These IMF's must be produced to a significant extent in non-equilibrium or dynamical processes and in geometrical locations at the intermediate system that are characterized by low gradients of the Coulomb potential. A simple scenario, in which IMF's are remnants of multiple rupture of a neck formed transiently between the reaction partners, appears to be compatible with the present data. Although analogous processes are known from collisions between classical water droplets, the discovery of this aspect of the dynamics of IMF emission in fast, peripheral and, presumably, non-adiabatic interactions of two heavy ions presents an unexpected challenge of the current understanding of intermediate-energy heavy-ion reaction mechanisms.

The above $4 \pi$ measurements employed the Rochester 960- $l$, liquid-scintillator neutron multiplicity meter (NMM), a relatively small detector, designed and built for heavy-ion reaction studies in a wide range of bombarding energies, from fusion reactions near the barrier to intermediate-energy collisions. The detector design and performance are discussed in some detail, along with methods of its energy and efficiency calibration. Methods of processing signal streams generated by the NMM in response to multi-neutron reaction events are presented. The detector is designed to have a high sensitivity to neutrons emitted from slow-moving reaction products, such as 
TLF's, while most of the neutrons emitted from fast-moving sources are allowed to escape undetected. In addition to the neutron multiplicity information, several experiments have exploited the prompt-response signal of the NMM, which is a measure of the total kinetic energy of all neutrons entering the detector in a reaction event. These observables relate to the total amount of kinetic energy of relative motion dissipated in a reaction, which is a function and, therefore, a measure of the collision impact parameter. A preliminary calibration of the prompt-response energy signal has been achieved using $\gamma$-sources and $\gamma$-rays produced in the capture of thermal neutrons by Gd nuclei immersed in the scintillator liquid. The detector has been used successfully both in low-energy fusion reactions and in the $\mathrm{E} / \mathrm{A}=28 \mathrm{MeV}{ }^{209} \mathrm{Bi}+{ }^{136} \mathrm{Xe}$ reaction discussed above.

In preparation of multi-detector experiments using a liquid-scintillator neutron spectrometer and/or CsI(Tl) detector arrays, planned for the future, the simple concept of a very low-cost, but high-quality pulse-shape discrimination circuitry has been developed. This circuitry is used in conjunction with two commonly gated currentintegrating analog- -digital converters. The timing of the gate signal is uncritical and, hence, the pulse-shape information is obtained in a highly reproducible fashion. The pulse shape information has been employed successfully for the identification of the species of particles entering different types of detectors. Data are presented for application in $n-\gamma$ discrimination with NE213 detectors and for the identification of light charged particles $(p, d, t, \alpha)$ with CsI(T) scintillation counters.

A simple, one-transistor preamplifier stage has been developed and tested as enhancement of commercially available photo-multiplier (PM) divider chains. It produces fast PM anode signals, to be used in the generation of logical signals, of a pulse height making further amplification unnecessary. The circuit is built into the PM base housing and is operated in series with the divider chain. It has already been tested successfully in several applications and has been implemented in the SuperBall PM bases.

Previous measurements of associated neutrons in heavy-ion reactions have demonstrated the usefulness of a measurement of the capture time of each of possibly a large number of neutrons emitted in a reaction event, in addition to the mere multiplicity of neutrons and their prompt total kinetic-energy signal. Such a measurement is to be done with any of the 5 segments of the SuperBall. In order to minimize the complexity 
of the electronics setup required for this type of measurement and the time needed to adjust it, a five-channel CAMAC event handler was designed, constructed and tested successfully. This CAMAC module combines the functions of a multi-stop time-to digital converter (TDC) with those of a bank of fast multiplicity counters, supervised by an internal controller. It is able to digitize information in the signal streams generated by the five individual segments of the SuperBall neutron detector. The module, built to a large extent in programmable-array logic technology, is capable of recording, event by event, with 250-ns precision and only $25 \mathrm{~ns}$ dead time, the times of up to 512 signals for each of the SuperBall segments. Independently, the module counts the multiplicities of signals arriving at any of five inputs and the multiplicities of logical OR signals for five selected patterns of input signals. Its implementation reduces significantly the number and cost of electronic modules required for the measurement.

An account is given of the status of the SuperBall project, in which a $16,000-l 4 \pi$ neutron detector is being built for calorimetric studies of intermediate-energy heavy-ion reactions. The design of this large detector allows it to accommodate complex setups of charged-particle arrays, such as DWARF or MINIBALL $4 \pi$ arrays, in its internal scattering chamber. Such detector combinations allow one to measure with full coverage all particles emitted in a complex heavy-ion reaction, as is necessary for a reconstruction of event history. The construction of the SuperBall basic mechanical structure nears completion. Work is currently concentrating on other mechanical elements supplementing this structure, such as scattering chamber, scintillator fluid handling system, and vacuum system. In parallel, work is progressing on completing Integrated Logic modules for signal processing described above.

In order to arrive at efficient and practical solutions for some constructional details of the SuperBall, a computer code was written for simulating the propagation and collection by photo-multipliers (PM) of the light generated in the detector segments in reaction events. The code can handle a wide variety of detector shapes. One of the important questions answered by the calculations, made possible with the code, pertained to the trade-off between light collection efficiency and sensitivity of the PM arrays to the positions of neutron interaction sites in the various SuperBall segments. The solution adopted for the actual detector combines a high collection efficiency with good position resolution. It consisted in a cladding of the interior surfaces of the SuperBall segments with diffusely reflecting sheet Teflon. 
In order to choose an effective, yet economical liquid scintillator for the SuperBall neutron calorimeter, samples of Gd-loaded scintillator supplied by several different manufacturers were subjected to a number of test measurements. Measurements carried out with relatively small test cells of liquid, addressed the total light output of each fluid, the light attenuation length, and the achievable energy resolution. Further considerations included the reflectance of the types of coating of the interior walls of the SuperBall tanks, the chemical compatibility of the scintillators with the materials used in the construction of the tank segments, and the compatibility of its light output with different types of PM's and associated electronics. As a by-product, these measurements also confirmed that, over a period of decades, a liquid scintillator liquid suffers only a small deterioration in total light output and essentially no decrease in attenuation length, if proper procedures for storage and iransfer of the liquid are followed.

Finally, as part of a Rochester high school Senior Study Project, a feasibility study was carried out of the suitability of the negative- and positive-muon components of the cosmic radiation for purposes of a calibration of the SuperBall high-energy light output signals. The study showed that it is possible to stop negative muons in a macroscopic target. However, due to the hard muonic energy spectrum, the muon stop rate is relatively low. Further tests will be needed to explore whether the $\mu^{+}$decay positrons can be used for a calibration of large-volume scintillator detectors.

During the Academic Year 1992-1993, the principal investigator devoted approximately $50 \%$ of his time to research supported by this grant, increasing to $100 \%$ during summer of 1993. The Senior Faculty Consultant (J.R.H.) spent approximately $15 \%$ of his time on research supported by this grant. The Senior Research Associate (J.T.), the research associates and scienuific visitors spent $100 \%$ of their efforts on such research projects. Mr. Bernd Quednau, senior graduate student, worked full time on grant research until end of the fall term of 1992, when he submitted his Ph.D. thesis. He has since accepted a research fellowship and began work at the French heavy-ion research center GANIL. The chemistry and physics graduate students Shawn Baldwin and Xiaofei Zhao worked full time on grant research during the entire past grant period. During the fall of 1992, Sharon Masserant spent $100 \%$ of her time on grant research, but only 50\% of her time during the spring term of 1993, when she was supported by a teaching assistantship. After having received her M.S. degree, she will continue her education in chemical engineering at Rutgers University. During the summe: months of 1993, all students worked full time on grant research projects. In addition, our group 
has hosted Ms. Karen Ho, a Rochester high school student, during her senior research study, and Ms. Eva Katta, another Rochester high school student and recipient of a SEED fellowship sponsored by the American Chemical Society.

To the best of our knowledge, we have complied with all grant requirements. It is a pleasure to acknowledge the direct support of our research program by the United States Department of Energy. In addition, we are grateful to the National Science Foundation for having provided indireci support through the use of the facilities of the Nuclear Structure Research Laboratory of the University of Rochester. 
III. Research Program 
John R. Huizenga

\section{Department of Chemistry and Nuclear Structure Research Laboratory University of Rochester, Rochester, New York 14627, USA}

Some may be curious to hear what has transpired, if anything, in the cold fusion saga since July 1,1991 , the date when the material in my book on cold fusion went to press. As most of you probably have anticipated, claims of successful cold fusion experiments have continued to appear in media stories, preprints, conference proceedings and peer-reviewed journals. In fact, some of the recent events associated with cold fusion could even be classified as downright sensational. Take, for example, the several new reports claiming enormous amounts of excess heat with ordinary light water -- the early workers in the field of cold fusion had used light water in control cells and stated categorically that light water cells gave zero excess heat. Now all at once a wave of claims are made that the energy gain and probability of success are both much enhanced with light water. One light-water group rejected the notion that cold fusion is due to nuclear reactions; proposing instead a new chemical theory with a squeezed ground state of hydrogen as a replacement for the time-tested quantum mechanics. Some have violated the conservation of energy in an attempt to rationalize cold fusion. Others have postulated element transmutation in the chemistry laboratory, including even the preposterous claim of making gold, fulfilling the alchemist's dream.

Equally striking was the stock market response to a positive cold fusion claim made in a press conference (during the Third International Conference on Cold Fusion held in Nagoya, Japan) by E. Yamaguchi, a scientist at the Nippon Telegraph and Telephone (NTT) Corporation. On the basis of Yamaguchi's unverified experiment, NTT's stock rose $11 \%$ amounting to a paper profit of approximately eight billion dollars. New companies, venture capital, profits, the stock market, competitors, proprietary research, trade secrets, etc. are now all part of the cold fusion saga. The addition of money and forprofit motives to the already complicated mixture of extraordinary experimental claims

\footnotetext{
* Excerpts from a new paperback edition of my book on cold fusion to be published by Oxford University Press in the fall of 1993.
} 
and proliferation of far-out theories to explain these claims complicates immeasurably the verification process.

Essentially all mainstream scientists had abandoned cold fusion research by the time my original manuscript went to press. However, a hard core of enthusiasts remained. Two examples of their recent claims will serve to characterize the present state of the cold fusion episode.

\section{Light Water Revolution}

Deuterium, the magical fuel in cold fusion experiments, is now obsolete according to some avid believers in cold fusion. Replace heavy water $\left(\mathrm{D}_{2} \mathrm{O}\right)$ solutions and palladium cathodes with light water $\left(\mathrm{H}_{2} \mathrm{O}\right)$ solutions and nickel cathodes and the energy gain and probability of success with cold fusion cells are greatly enhanced. This is the new gospel preached by a subset of cold fusion advocates. One couldn't wish for a more common and abundant fuel than $\mathrm{H}_{2} \mathrm{O}$. After all, deuterium is a very minor isotope $(0.015 \%)$ of the element hydrogen and, hence, is present in only trace amounts in water. Now, so it is claimed, one can utilize the major isotope (99.985\%) of the element hydrogen, and cold fusion is, therefore, a potentially much more spectacular energy source. The successful light water 'cold fusion' experiments were highly acclaimed by Business Week (March 2 and October 26, 1992) and the Wall Street Journal (October 19, 1992), news media often used by proponents to publicize their claims.

But don't rush out to spend your savings on light-water-stocks just yet. The above doctrine is heresy to another subset of the cold fusion community. Fleischmann and Pons and many other of their supporters have stated repeatedly in their publications that excess heat is obtained only with heavy hydrogen or deuterium. Their proof that $D+D$ nuclear fusion is occurring in their cells is based on their experimental evidence that no excess heat and nuclear products are observed when deuterium is replaced by ordinary light hydrogen. Hence, it is hard for this group to swallow the claim that their light water control cells are superior heat producers. No wonder then that this segment of the cold fusion community has, at least privately, written off the light water results as quackery. At the most recent (October 21-25, 1992) international meeting on cold fusion, however, there was no public criticism of the light water claims, leaving the impression that water was the critical 
ingredient, independent of whether it was light or heavy water. Although Mills and Kneizys ${ }^{*}$ were not the first to claim excess heat with light water cells, it was their paper [Fusion Technology 20, 65 (1991)] that triggered a wave of claims of successful cold fusion experiments with light water or ordinary hydrogen gas. This paper claims fantastic success by electrolysis of a solution of potassium carbonate in light water with a nickel cathode. Thirteen experiments, with the calibration heater off, gave an average excess power of $240 \%$, and sixteen experiments, with the calibration heater on, gave an average excess power of $1150 \%$. The energy gain was a factor of 5 superior with the calibration heater on! This result should have served as a cautionary signal. More important, none of the data in their Table I includes errors, and without these, most scientists would question the validity of such data.

The major portion of the Mills-Kneizys paper is devoted to theory, and unusual theory it is. First of all, they reject the dogma accepted by essentially all cold fusion proponents that cold fusion is powered by room temperature nuclear reactions. Instead, Mills believes that quantum mechanics is incorrect and he offers his theory as a replacement. The excess energy released during electrolysis of potassium carbonate solutions $\left(\mathrm{H}_{2} \mathrm{O}\right)$ arises in this model from the relaxation of hydrogen to a new shrunken orbit.

\section{Cold Fusion for Profit}

Cold fusion advocates have by now fostered several new companies. Here I will describe the formation of one such company. On August 10, 1992, Ronald A. Brightsen, Chief Executive officer and Russell George, President, announced the formation of Clustron Science Corporation (CSC), headquartered in Vienna, Virginia. CSC promised to introduce innovative commercial applications in the field of cold fusion. The press release stated that the corporation has made a breakthrough in nuclear science and it will capitalize on the business opportunities in the emerging field of new nuclear science. The essence of the new science was a nucleon cluster model (NCM) which was claimed to provide "a comprehensive explanation for both nuclear fission and cold fusion." Two of CSC's officers, Eugene F. Mallove, Vice President of Research, and Jed Rothwell, Vice

\footnotetext{
* Randell L. Mills is president of HydroCatalysis Power Corporation in Lancaster, PA; Steven P. Kneizys is in the Academic Computing Department of Ursinus College, Collegeville, PA.
} 
President, are co-founders of Cold Fusion Research Advocates. The Virginia company's claims, although reminiscent of those of the Cold Fusion Research Advocates, are even more exotic.

CSC received start-up capital from a venture capital firm and a group of investors (Washington Post, August 11, 1992). The addition of a for-profit motive to cold fusion has introduced a new dynamic to the; equation that shifts the equilibrium away from verification toward capital, technology and related issues. The press release contained a list of sixteen supporters (along with short resumes) of the nucleon cluster model (NCM), the foundation of the new Clustron Science Corporation. Within days it became known that the names of the so-called supporters had been used without their knowledge. Upon being contacted, eleven of these scientists repudiated the model. Of the remaining five, two could not be contacted and two are non-scientists [Nature, 358, 626 (1992)].

Those following the cold fusion saga will be interested to learn about the "breakthrough in nuclear science" on which Clustron Science Corporation is founded. Two of the corporation's founders and officers (Ronald A. Brightsen and Eugene F. Mallove) described in a manuscript submitted to Fusion Technology, August 9, 1992, how the nucleon cluster model (NCM) "explains" cold fusion. The abstract of this manuscript states:

The three most significant features of the NCM cold fusion model are: (1) Its ability to provide a unified explanation for excess heat of nuclear origin in both dominantly $\mathrm{D}_{2} \mathrm{O}$ and dominantly $\mathrm{H}_{2} \mathrm{O}$ electrochemical cells; (2) Its ability to explain reaction products: neutrons, tritium, and helium; (3) Its ability to explain why nuclear reaction end products commensurate with observed excess heat have not been found inside cold fusion cells. The explanation lies in the unsuspected structure of the proton, which is required by the NCM of the nucleus as the bridge between matter and antimatter.

The authors postulated two possible heat producing reactions and I'll include here only the first which they consider to be the more likely.

$$
\begin{aligned}
{[\mathrm{NP}+\overline{\mathrm{N}} \overline{\mathrm{P}}]+\mathrm{NP} } & \rightarrow \mathrm{PNP}+3.72 \mathrm{GeV}^{*} \\
\mathrm{p}+\mathrm{D} & \rightarrow{ }^{3} \mathrm{He}+5.49 \mathrm{MeV}
\end{aligned}
$$

\footnotetext{
* This value was calculated incorrectly by multiplying $931 \mathrm{MeV}$ by 4 . However, this error is relatively small and is not important for the discussion here.
} 
The NCM of Brightsen and Mallove requires that the well-studied $\mathrm{p}+\mathrm{D} \rightarrow{ }^{3} \mathrm{He}+\gamma$ reaction, as given by (B), he rewritten as (A) due to the complexity introduced by the unexpected structure of the proton $[\mathrm{PNP}+\overline{\mathrm{N}} \overline{\mathrm{P}}]$, where $(\overline{\mathrm{N}} \overline{\mathrm{P}})$ is an antimatter cluster of the normal (NP) cluster.

The authors state why this particular proton structure was postulated in rather categorical terms, namely:

It is because this structure most clearly explains the proton in the framework of the NCM, i.e., $[\mathrm{PNP}+\overline{\mathrm{NP}}]$, where mathematically, $3+(-2)=1$. The NCM forces us to postulate such a structure.

With my calculator in hand, I agree at least with the equation, 3-2 = 1! However, the assumption that the proton [PNP $+\overline{\mathrm{N}} \overline{\mathrm{P}}]$, with a mass of $940 \mathrm{MeV}$, has components $\mathrm{P}, \mathrm{N}$, $\overline{\mathrm{N}}$ and $\overline{\mathrm{P}}$ which each contribute some $940 \mathrm{MeV}$ in the laboratory violates the conservation of mass and energy. Once Mallove's fictitious proton [PNP $+\overline{\mathrm{N}} \overline{\mathrm{P}}]$ is created, how is it used to "explain" all existing cold fusion experiments? First, why does cold fusion occur in both heavy and light water cells? Brightsen and Mallove solve this problem by postulating heat producing reactions of the type shown above. Since both hydrogen and deuterium are reaction participants, the success with electrolysis of heavy water depends on small amounts of light water in $\mathrm{D}_{2} \mathrm{O}$, and the success with light water depends on trace amounts of deuterium in ordinary $\mathrm{H}_{2} \mathrm{O}$. Hence, the conclusion that the "dominant heat reaction in cold fusion is actually the reaction of a proton and a deuteron" is based on nothing more than an a priori postulate.

Secondly, what does the nucleon cluster model predict about the energy release and the fusion products? The GeVs of energy release associated with a proton reacting with a deuteron (reaction $\mathrm{A}$ ) is due to a matter-antimatter annihilation reaction because of the "hidden antimatter cluster $(\overline{\mathrm{N}} \overline{\mathrm{P}})$ in the structure of the proton." The authors state that the resulting very energetic ${ }^{3} \mathrm{He}$ nuclei will mostly leave the cell. This they say explains why the intensity of fusion products are not commensurate with the observed heat! Brightsen and Mallove don't realize that even if their far-out postulate were correct, the conservation of momentum requires recoil nuclei with large energies, e.g., palladium nuclei with well over $100 \mathrm{MeV}$ of energy. Such energetic recoil nuclei would produce copious intensities of secondary products, e.g., n, p, $\mathrm{x}$-rays and g-rays, which are easily measurable. So the 
"hidden fusion product" postulate is seriously flawed. Those trained in science will also notice that the postulated energy of reaction (A) exceeds the summed masses of the reactants, hydrogen and deuterium, thereby violating the conservation of mass and energy according to the Einstein relation, $\mathrm{E}=\mathrm{Mc}^{2}$.

With release of their cold fusion manuscript based on the NCM, Brightsen and Mallove included sixteen self-serving questions and answers. Examples are the following: "Q: What will happen to the hot fusion program? A: It won't be needed. Q: We have heard rumors about transmutation of mercury to gold. What can you tell us? A: The NCM model is completely consistent with transmutation. Q: What will happen to the oil industry? A: We believe that there will be a gradual change to cold fusion from petroleum-based fuels over a period of many years."

\section{Conclusion}

After nearly four years and the expenditure of many tens of millions of dollars worldwide, we still only have unsubstantiated and fragmentary claims of watts of excess heat generated from light and heavy water (and $\mathrm{H}_{2}$ and $\mathrm{D}_{2}$ gases) by some unknown mechanism reported to have a nuclear origin. To this date, not a single, well-controlled and reproducible experiment has been reported where corresponding amounts of excess heat and nuclear reactions products are reliably measured. This is the current status of cold fusion. Hence, I conclude now, as I did in July, 1991, that the claim that cold fusion is a nuclear process producing watts of excess power, without commensurate nuclear reaction products, is a chimera and qualifies as pathological science. At best, the cold fusion fiasco may lead to new information on materials and energy storage, but even this has not heen established at this time. Furthermore, there is still no persuasive evidence that any nuclear reaction products have been positively identified in cold fusion experiments in very low-level background environments. Regrettably, the idea of producing useful energy from room-temperature nuclear reactions is an aberration. 
J. L. Wile, D. L. Coffing, E. T Bauer, A. L. Michael, and M. A. Doerner Department of Chemistry Ball State University, Muncie, IN 47306

\author{
S. P. Baldwin, B. M. Szabo, B. Lott, B. M. Quednau, J. Töke, \\ and W. U. Schröder \\ Department of Chemistry and Nuclear Structure Research Laboratory \\ University of Rochester, Rochester, NY 14627, USA \\ R. T. de Souza \\ Department of Chemistry and Cyclotron Facility \\ Indiana University, Bloomington, IN 47408
}

\begin{abstract}
Multiplicity distributions of neutrons and energy spectra of light charged particles have been measured in coincidence with evaporation residues from the ${ }^{32} \mathrm{~S}+{ }^{118,124} \mathrm{Sn}$ fusion reactions at $E_{l a b}=130-180 \mathrm{MeV}$. The experimental data are found to be in agreement with the results of statistical-model calculations, provided $a=A / 12 \mathrm{MeV}^{-1}$ is assumed for the effective level density parameter for all nuclides in the decay path. Simulation calculations have further shown that the "anomalously" low value of the effective level density parameter cannot be explained by the population of superdeformed bands lowering effectively the thermal excitation energy of the systems involved.
\end{abstract}




\section{INTRODUCTION}

In recent studies [1] of the decay of the ${ }^{146,152} \mathrm{Gd}$ compound systems formed in the ${ }^{28} \mathrm{Si}+{ }^{118,124} \mathrm{Sn}$ fusion reactions, marked differences were found between the decay patterns of the two isotopes, in agreement with the results of several earlier studies $[2,3]$. While the earlier results had been interpreted tentatively $[2,3]$, as arising from the effects of superdeformation, a phenomenon known to realize in the region of nuclides involved [4-9], the more complete set of experimental evidence reported in [1] appeared to be incompatible with such an interpretation. Instead, it has been shown [1] that the differences in the decay patterns of the two systems can consistently be explained by assuming different level density parameters $a$ for the two decay chains. While a "conservative" value of $a=A / 8 \mathrm{MeV}^{-1}$ allows one to explain, within the framework of the statistical model, the gross features of the experimental data on the decay of the lighter system ${ }^{146} \mathrm{Gd}$, a somewhat "anomalous" value of $a=A / 12$ $\mathrm{MeV}^{-1}$ was found neccessary in order to explain simultaneously the "anomalously" low average neutron multiplicity, the elevated variance of the neutron multiplicity distribution, and the "anomalously" hard energy spectrum of neutrons evaporated from the heavier ${ }^{152} \mathrm{Gd}$ system.

In order to extend the systematics of particle decay patterns of compound systems in the region of nuclides known to exhibit superdeformation, a study similar to that reported in Ref. [1] was carried out for the fusion reactions ${ }^{32} \mathrm{~S}+{ }^{118,124} \mathrm{Sn}$ at $\mathrm{E}_{\text {lab }}=130$ - $180 \mathrm{MeV}$. The presence of superdeformed bands has been established $[10,11]$ for the ${ }^{151,152} \mathrm{Dy}$ nuclides populated in the decay of the ${ }^{156} \mathrm{Dy}$ compound system formed in the second reaction. Therefore, a comparison of the decay patterns of the two systems studied can shed additional light on the possible role of the superdeformation in altering the balance of phase space available to various particle decay modes.

The experimental procedure is described in Section II, while experimental results and discussion are presented in Section III. Section IV summarizes the conclusions of 
this work.

\section{EXPERIMENTAL TECHNIQUE}

The experimental setup is shown in Fig. 1. The experiment was performed at the University of Rochester Nuclear Structure Research Laboratory. Beams of 130,155 , and $180 \mathrm{MeV}^{32} \mathrm{~S}$ ions from the MP Tandem Van de Graaff accelerator bombarded targets of $150 \mu \mathrm{g} / \mathrm{cm}^{2}$, isotopically enriched ${ }^{118,124} \mathrm{Sn}$ on $10 \mu \mathrm{g} / \mathrm{cm}^{2}$ carbon backings, placed in the center of the Rochester Neutron Multiplicity Meter (NMM) [12]. Massive reaction products, including evaporation residues (ER) and projectilelike-fragments (PLF), were detected in two 300- $\mu \mathrm{m}$ thick multistrip silicon detectors placed at small angles, $60 \mathrm{~cm}$ downstream, on either side of the beam. Combined, the multistrip detectors spanned an angular range from $2^{\circ}$ to $8^{\circ}$. Light charged products were measured using seven $1.4-\mathrm{cm}$ thick $\mathrm{CsI}(\mathrm{Tl})$ scintillation detectors, placed at various angles, and at a distance of approximately $9 \mathrm{~cm}$ from the target. Three of these detectors were placed in the common "recoil" plane with the multistrip detectors, at angles of $\Theta=20^{\circ},-40^{\circ}$, and $-20^{\circ}$, while the remaining four $\operatorname{CsI}(\mathrm{Tl})$ detectors were placed "out-of-plane", at angles $\left(\Theta=-20^{\circ}, \phi=33.5^{\circ}\right),\left(\Theta=20^{\circ}, \phi=33.5^{\circ}\right),\left(\Theta=-20^{\circ}\right.$, $\left.\phi=12.3^{\circ}\right)$, and $\left(\Theta=20^{\circ}, \phi=12.3^{\circ}\right)$. Additionally, two silicon-detector telescopes were placed at angles of $\Theta=40^{\circ}$ and $\Theta=-70^{\circ}$, also to measure light charged products. These telescopes were comprised of three elements each: a 50- $\mu \mathrm{m}(\Delta \mathrm{E} 1)$ and a $300-\mu \mathrm{m}$ $(\Delta E 2)$ transmission detector, as well as a $10-\mathrm{mm}$ stop detector (E). They covered a significantly smaller solid angle than each of the CsI(Tl) detectors and were mainly used as a device to cross-check the critical energy calibration of the CsI(Tl) detectors.

Neutrons were detected using the 960-liter, highly-efficient liquid-scintillator "multiplicity meter" NMM [12]. The detection efficiency of this detector for neutrons depends on neutron energy but was on average approximately equal to $76 \%$. This efficiency was calculated using a modified version of the computer code DENISW 
[13] and was verified experimentally using the well-known standard of a ${ }^{252} \mathrm{Cf}$ fission neutron multiplicity distribution.

\section{RESULTS AND DISCUSSION}

In Figs. 2-5 results of the experiment are compared to various statistical-model calculations. Fig. 2 shows the first $(\bar{m})$ and second $\left(\sigma_{m}^{2}\right)$ moments of the multiplicity

distributions of neutrons from the ${ }^{32} \mathrm{~S}+{ }^{118} \mathrm{Sn}$ reaction as functions of bombarding energy. In this figure, the experimental data are represented by circles with error bars, while the results of statistical-model calculations, performed with the computer code PACE [14] under three different sets of assumptions, are shown as continuous curves. The dashed and solid curves illustrate the results of calculations in which all relevant statistical-model parameters, with the exception of the scaling factor $\mathrm{A} / a$ of the effective level density parameter $a$ for a nuclide of mass number $A$, were taken from systematics incorporated as the code's default values. As seen from Fig. 2, the calculations assuming a systematic value of $a=A / 8 \mathrm{MeV}^{-1}$ for the level density parameter (dashed lines) reproduce correctly the average multiplicities of neutrons, however, they systematically underpredict the widths (variances) of the multiplicity distributions. At the same time, significantly reduced, "anomalous" values of the level density parameters of $a=A / 12 \mathrm{MeV}^{-1}$ are seen to result in a reasonably good agreement (solid lines) between model calculations and both, the first and second moments of the experimental neutron multiplicity distributions. The dash-dotted lines represent results of PACE calculations in which a smooth-cut-off distribution of angular momenta, with a diffuseness of $\Delta l=15 \hbar$, was assumed for the fused system, in addition to a level density parameter of $a=A / 12 \mathrm{MeV}^{-1}$. The assumption of a smooth cut-off, seen in Fig. 2 to improve the agreement between the measured and calculated variances at low bombarding energies, finds a theoretical justification in the well-known effect of fusion barrier fluctuations [15]. Quantitatively, however, the 
assumed diffuseness of $\Delta l=15 \hbar$ is somewhat larger than expected [15] from the $\Delta l$ systematics based on barrier fluctuations.

A similarly large reduction in the value of the level density parameter $a$ and a similarly large diffuseness in the angular momentum cut-off appear to be necessary to explain the averages and widths of the neutron multiplicity distributions measured for the heavier system ${ }^{32} \mathrm{~S}+{ }^{124} \mathrm{Sn}$. As seen from Fig. 3, showing experimental and theoretical results for this heavier system, the reduced value of the level density parameter of $a=A / 12 \mathrm{MeV}^{-1}$ (solid line) provides for a significantly better account of both first and second moments of the neutron multiplicity distributions than the "standard" value of $a=A / 8 \mathrm{MeV}^{-1}$ (dashed line), suggested by systematics. Like in the case of the lighter system, introduction of a smooth cut-off angular momentum distribution with a diffuseness of $\Delta l=15 \hbar$ into the model calculations improves the agreement between the results of these calculations (dash-dotted line) and the experimental data, at low energies.

In order to investigate whether collective effects, such as, e.g., those associated with superdeformation could provide an alternative explanation of the experimental data, not requiring an "anomalous" lowering of the value of the level density parameter $a$, an yrast line raised above the iquid-drop model yrast line was introduced into the PACE code, representing an increased collective energy of system. This yrast line was parameterized in the computer code in terms of two parameters, the critical spin $\left(\mathrm{J}_{c}\right)$ and the "excess" collective yrast energy $\left(\Delta E_{s d}\right)$, and whenever a (daughter) nucleus in the decay chain had a spin greater than $\mathrm{J}_{c}$, its liquid-drop yrast energy was increased by $\Delta E_{s d}$. These two parameters were then varied in a series of calculations, assuming always $a=A / 8 \mathrm{MeV}^{-1}$, to obtain agreement between the calculated and experimental first moments of the neutron multiplicity distribution. However, no common set of values of the two parameters $\mathrm{J}_{c}$ and $\mathrm{E}_{s d}$ was found suitable simultaneously for all bombarding energies. The "best fit" values of these parameters for the three bombarding energies of $\mathrm{E}=130,155$, and $180 \mathrm{MeV}$ were $\mathrm{J}_{c}=10,25$, and 10 , 
and $F_{\text {sdd }}=10,8$, and $10 \mathrm{MeV}$, respectively.

The results of these calculations, simulating possible effects of superdeformation, are illustrated by the dotted curves in Fig. 3. As can be seen from this figure, an assumption that the system decay chains are occasionally trapped in bands with unusuelly large collective energy allows one to obtain an agreement brtween the experimental values of average neutron multiplicities and their "model" values calculated using the systematic level density parameter of $a=A / 8 \mathrm{MeV}^{-1}$. At the same time, however, the widths of the neutron multiplicity distribution are seen to be even more severely underpredicted than they are in "standard" statistical-model calculations using the code PACE [14] and the same scaling, $a=A / 8 \mathrm{MeV}^{-1}$, of the level density parameter. Such an adverse effect of an increased collective energy (as simulated in the present study by the modified yrast line) on the calculated widths of the neutron multiplicity distributions is easily understood. An elevated yrast line reduces the thermal, intrinsic excitation energy of the decaying nucleus, and hence, fewer decay paths become available to the system. As a result, both the average neutron multiplicity and the width of the multiplicity distribution decrease, improving the agreement of the former, while deteriorating the agreement of the latter, with experimental first and second moments of the neutron multiplicity distributions, respectively.

An independent check of the validity of statistical-model parameters, found to be best suited for the present systems, is possible through a comparison of the model predictions for the ligt charged-particle spectra to the measured spectra. In particular, the slope parameters of these spectra are sensitive to the temperatures of the decaying systems, and can, hence, be used as a measure of the thermal enetgy and the level density parameter $a$ involved. Such comparisons between the results of calculations and experimental spectra are shown in Figs. 4 and 5. As seen from these figures, for both protons (Fig. 4) and $\alpha$-particles (Fig. 5), calculations in which $a=A / 12 \mathrm{MeV}^{-1}$ is assumed, provide the best account of the experimental data, similarly to what was observed in the case of the neutron data. Furthermore, an attempt to simulate the 
effects of superdeformation by an elevated yrast line results in an inferior overall agreement with the experiment, also for the charged-particle data.

\section{CONCLUSIONS}

The present analysis has shown that both the neutron and the light chargedparticle data measured in the fusion reactions ${ }^{32} \mathrm{~S}+{ }^{118,124} \mathrm{Sn}$ can consistently and well be accounted for by statistical-model calculations when a reduced value of $a=A / 12$ $\mathrm{MeV}^{-1}$ is assumed for the level density parameter, for all nuclides along the decay paths of the fused systems. This type of scaling of the level density parameter has in an earlier study been found compatible with the energy spectra and multiplicity distributions of neutrons from the ${ }^{28} \mathrm{Si}+{ }^{124} \mathrm{Sn}$ fusion reaction, but not with neutrons emitted in the reaction ${ }^{28} \mathrm{Si}+{ }^{118} \mathrm{Sn}[1]$. A simple simulation of the possible collective effects, such as those that could perhaps be associated with superdeformation phenomena, has shown that such effects do not find support in the "anomalous" gross features of neutron multiplicity distributions and of the shapes of the light chargedparticle spectra. These findings do, however, not rule out a common origin of the effects of superdeformation and of unusual particle decay patterns in the microscopic structure of the nuclides involved. A definite answer to these questions must await further theoretical analysis. 


\section{References}

[1] J. L. Wile, S. S. Datta, W. U. Schröder, J. Töke, D. Pade, S. P. Baldwin, J. R.

Huizenga, B. M. Quednau, R. T. de Souza, and B. M. Szabo, Phys. Rev. C47 2135 (1993).

[2] P. M. Stwertka, T. M. Cormier, M. Herman, and N. G. Nicolis, Phys. Lett. B150, 91 (1985).

[3] P. M. Stwertka, T. M. Cormier, N. G. Nicolis, and M. Herman, Phys. Lett. B161, 70 (1985).

[4] C. Cabot, H. Gauvin, H. Sergolle, P. Aguer, G. Bastin, J. P. Thibaud, H. Delagrange, and Y. Patin, Z. Phys. A322, 393 (1985).

[5] A. Ruckelshausen, R. D. Fisher, W. Kühn, V. Metag, R. Mühlhaus, R. Novotny,

T. L. Khoo, R. V. F. Janssens, H Grger, D. Habs, H. W. Heyng, R. Repnow, D. Schwalm, D. Duchne, R. M. Freeman, B. Haas, F. Haas, S. Hlavac, and R. S. Simon, Phys. Rev. Lett. 56, 2356 (1986).

[6] R. V. F. Janssens, R, Holtzmann, W. Henning, T. L. Khoo, K. T. Lesko, G. S. F. Stephans, P. C. Radford, A. M. van Denberg, W. Kühn, and R. M. Ronningen, Phys. Lett. B181, 16 (1986).

[7] D. J. Love, P. J. Bishop, A. Kirwan, P. J. Nolan, D. J. Thornley, A. H. Nelson, and P. J. Twin, Phys. Rev. Lett. 68, 158 (1986).

[8] S. Henss, A. Ruckelhausen, R. D. Fischer, W. Kühn, V. Metag, R. Novotny, R. V. F. Janssens, T. L. Khoo, D. Habs, D. Schwalm, D. Freeman, G. Duchne, B. Haas, F. Haas, S. Hlavac, and R. S. Simon, Phys. Rev. Lett. 60, 11 (1988).

[9] G. Smith, B. Haas, A. Alderson, I. Ali, C. W. Beausang, M. A. Bently, P. Dagnall, P. Fallon, G. de France, P. D. Forsyth, U. Huttmeier, P. Romain, D. Santos, P. J. Twin, and J. P. Vivien, Phys. Rev. Lett. 68, 158 (1992).

[10] P. J. Twin, et al., Phys. Rev. Lett., 57, 811 (1986).

[11] G. E. Rathke, R. V. F. Janssens, M. W. Drigert, I. Ahmad, K. Beard, R. R. Chasman, U. Garg, M. Hass, T. L. Khoo, H. J. Körner, W. C. Ma, S. Pilotte, P. 
Taras, and F. L. H. Wolfs, Phys. Lett. B209, 177 (1988).

[12] D. Pade, W. U. Schröder, J. Töke, J. L. Wile, R. T. de Souza, Phys. Rev. C43, 1288 (1991).

[13] J. Poitou and C. Signarbieux, Nucl. Instr. Meth. 114, 113 (1974).

[14] A. Gavron, Phys. Rev. C21, 230 (1980).

[15] R. Vandenbosch, Annu.Rev.Nucl.Part.Sci. 42, 447 (1992). 


\section{Figure Captions}

Fig. 1. The experimental setup featuring the Rochester NMM, seven CsI(Tl) light charged-particle detectors and two multistrip massive-fragment detectors.

Fig. 2. The first (upper) and second (lower) moments of the neutron multiplicity distribution for the ${ }^{32} \mathrm{~S}+{ }^{118} \mathrm{Sn}$ fusion reaction. The curves represent various statistical-model calculations discussed in the text.

Fig. 3. Same as Fig. 1 for the ${ }^{32} \mathrm{~S}+{ }^{124} \mathrm{Sn}$ fusion reaction. The dotted curve represents a crude simulation of effects of superdeformation in the deexcitation cascade.

Fig. 4. Energy spectra for protons emitted in the ${ }^{32} \mathrm{~S}+{ }^{118} \mathrm{Sn}$ reaction (left hand side) and the ${ }^{32} \mathrm{~S}+{ }^{124} \mathrm{Sn}$ reaction (right hand side) at the two highest bombarding energies.

Fig. 5. Same as Fig. 3, except for $\alpha$-particles. 


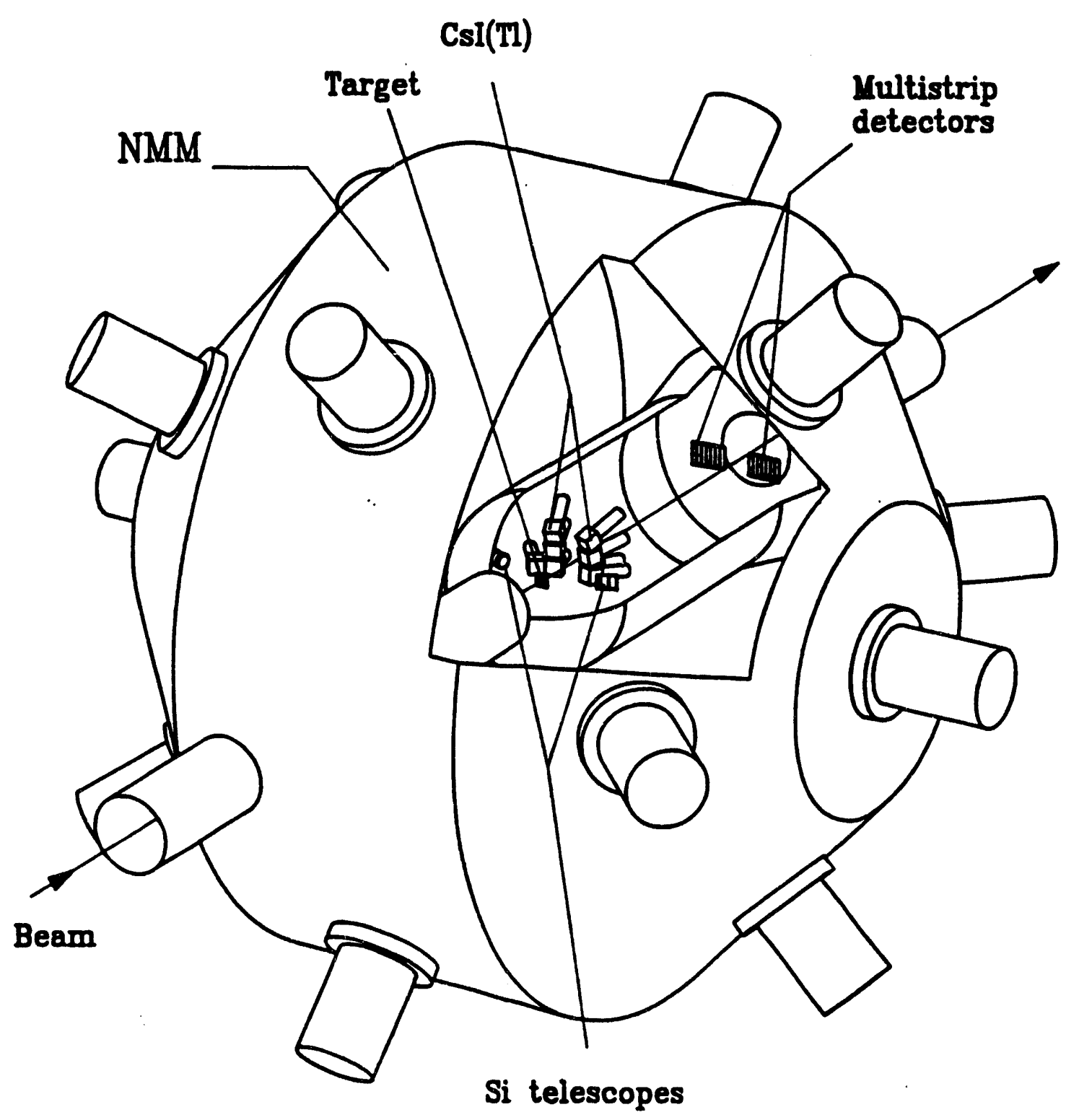

Fig. 1. 


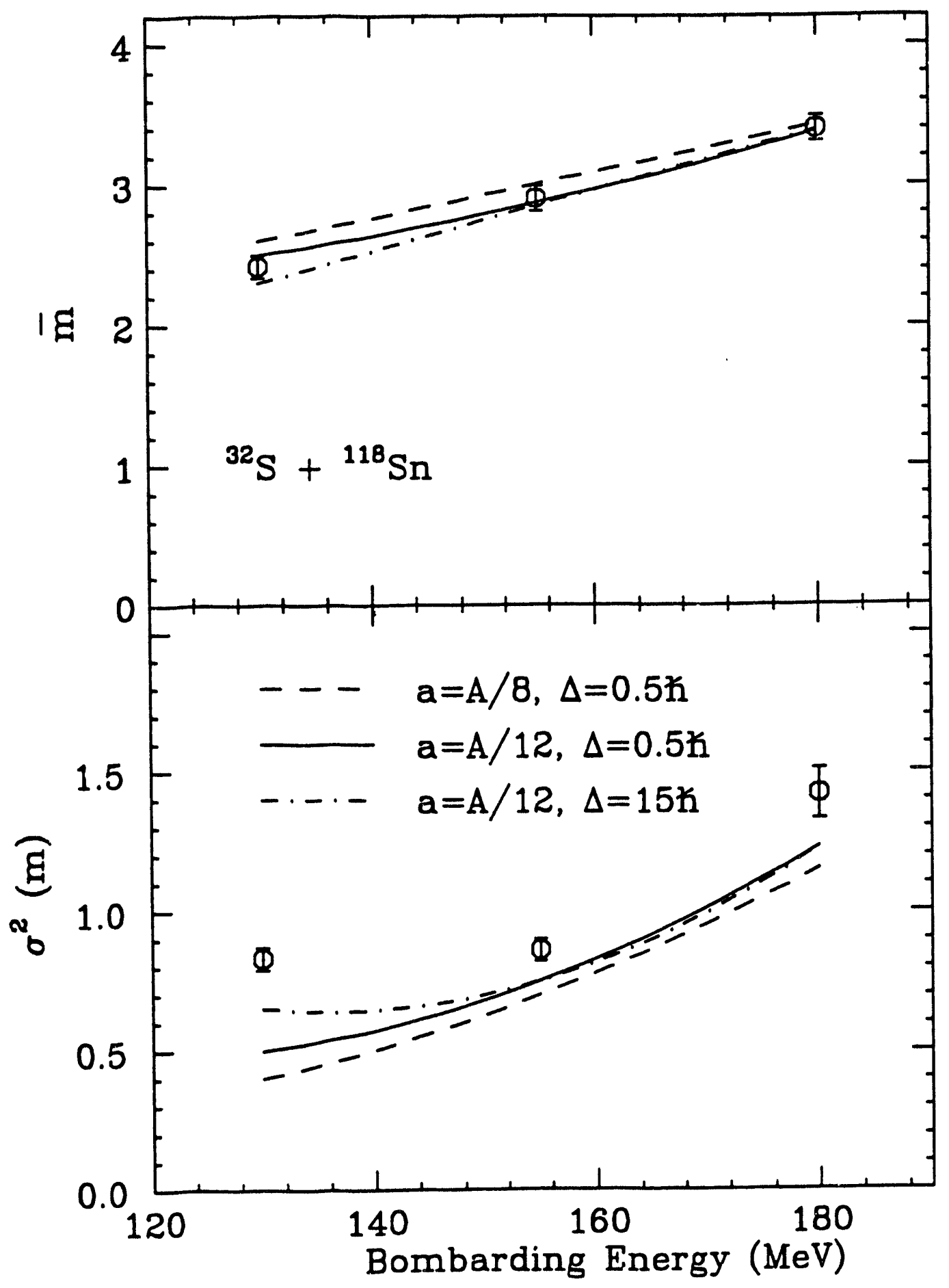

Fig. 2. 


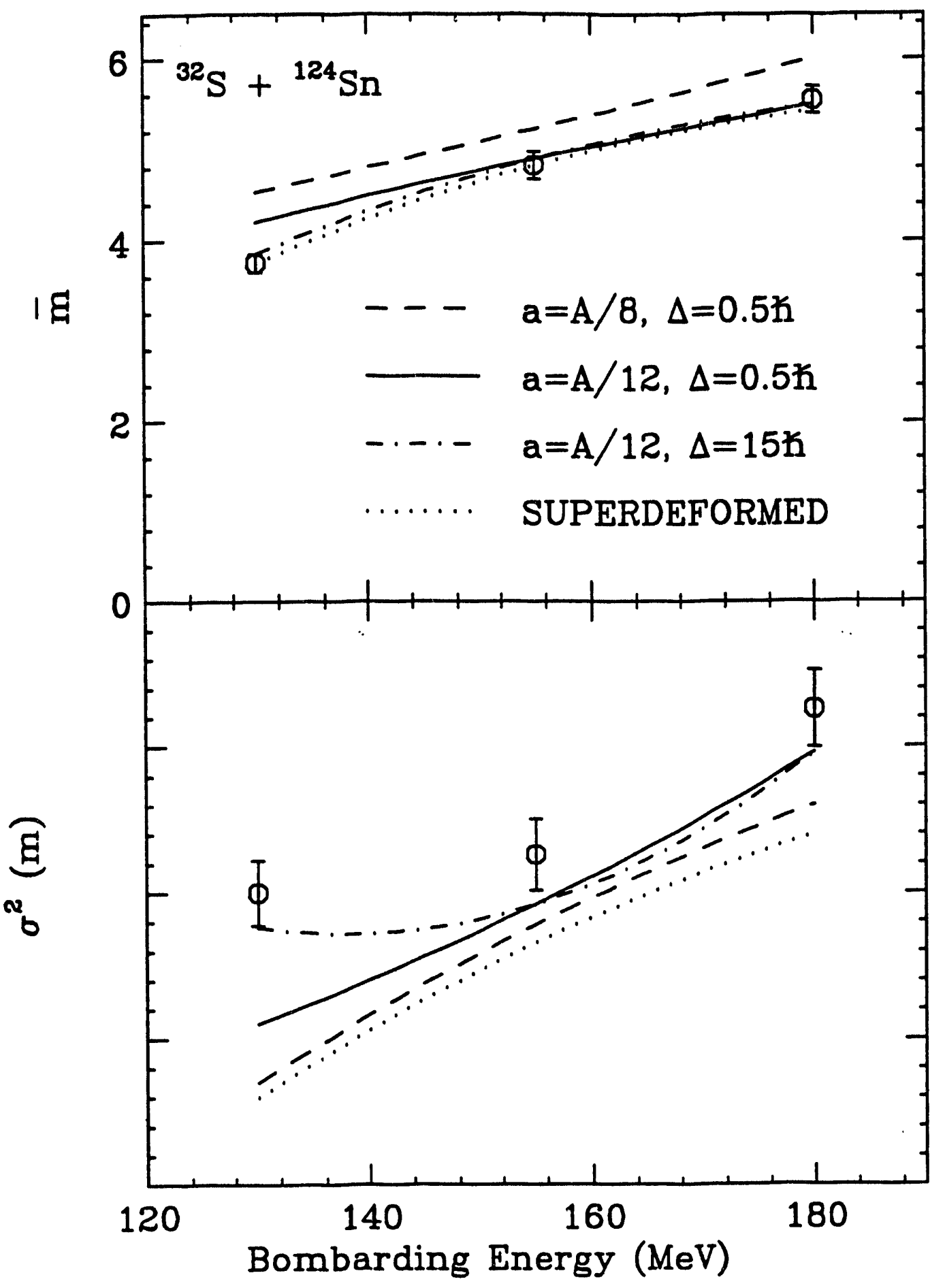

Fig. 3. 


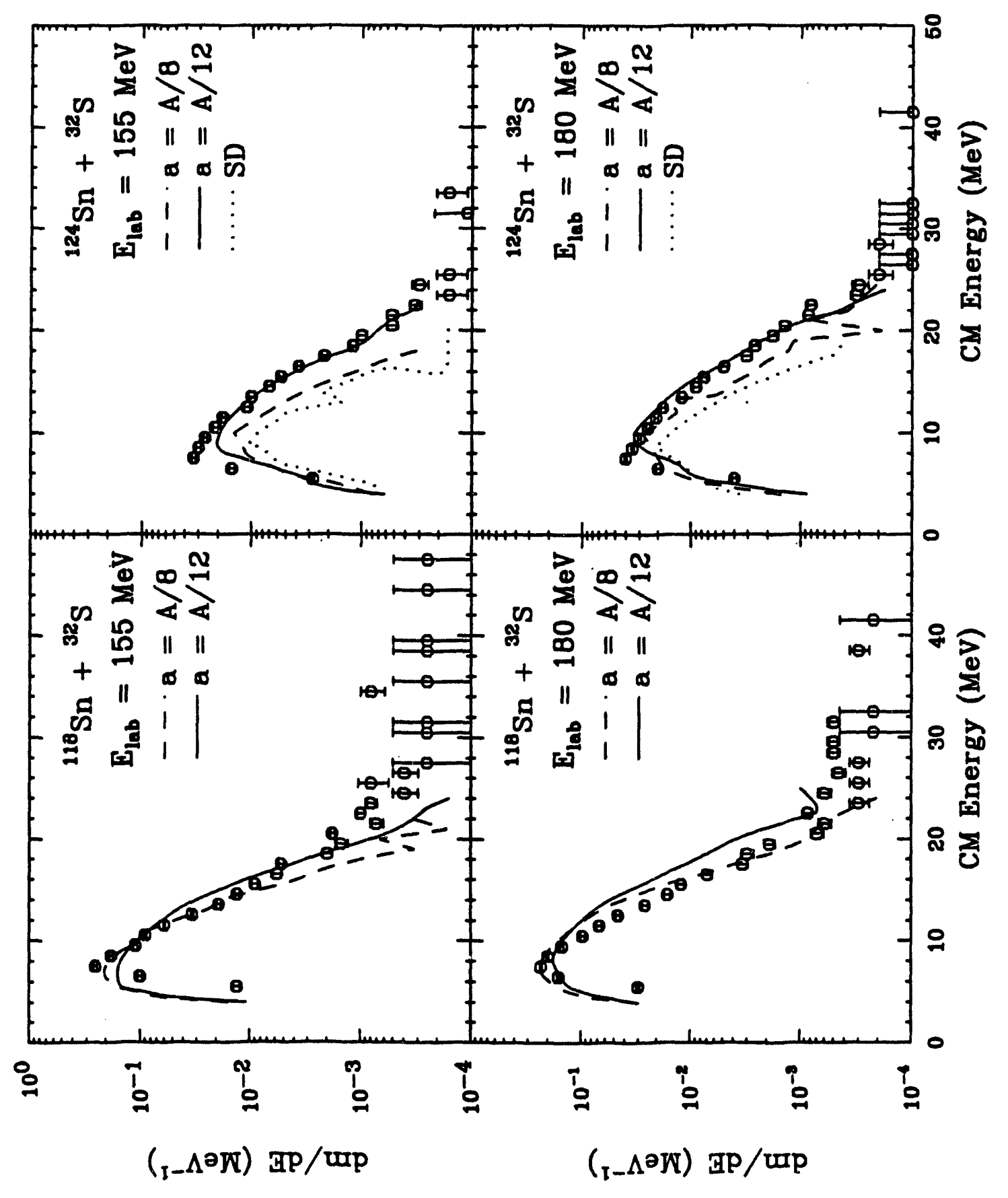

Fig. 4. 


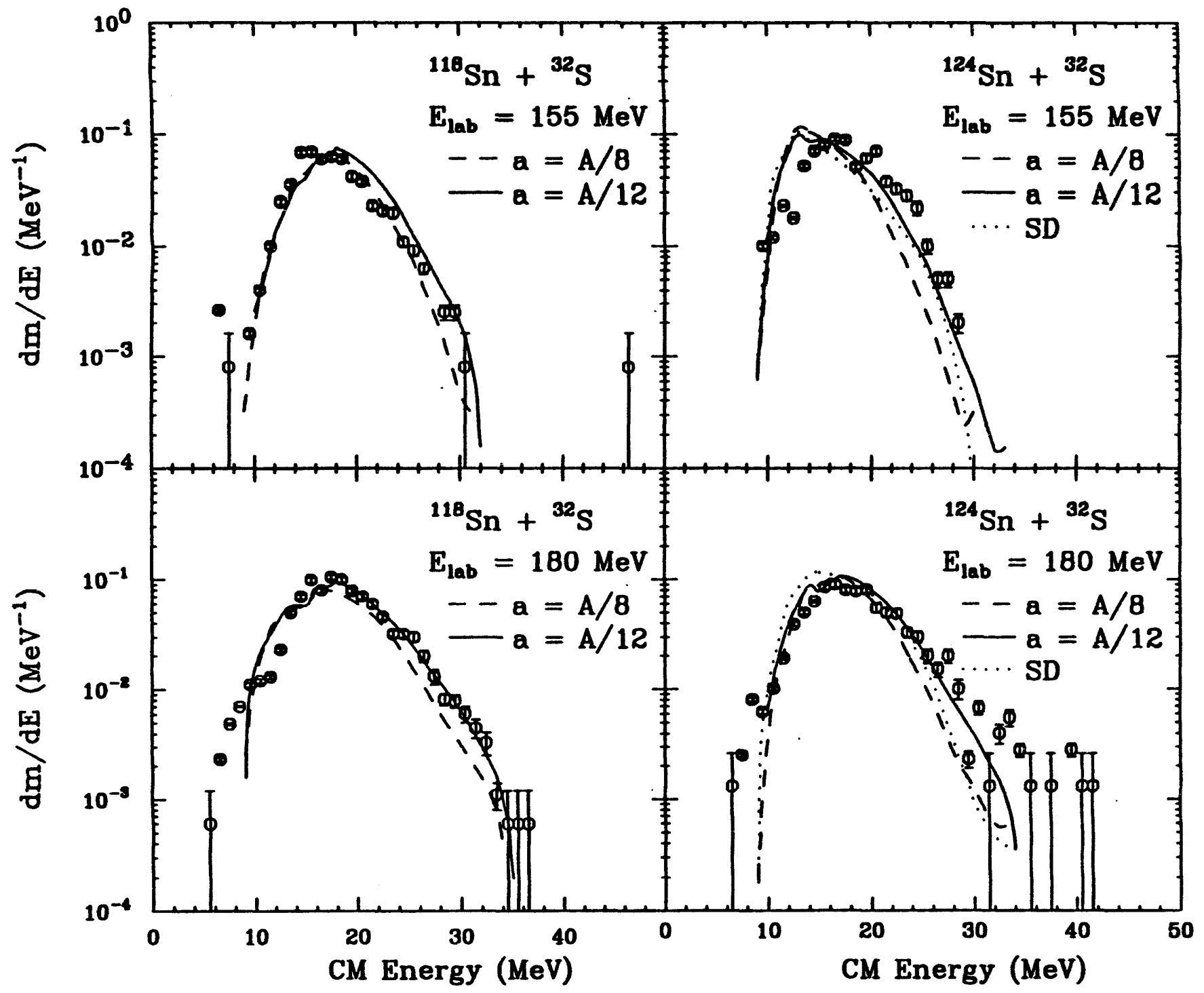




\title{
Unexpected Features of Reactions Between Very Heavy Ions at Intermediate Bombarding Energies
}

\author{
W.Udo Schröder \\ Department of Chemistry and Nuclear Structure Research Laboratory \\ University of Rochester, Rochester, New York 14627, USA
}

\begin{abstract}
Exclusive measurements of massive projectile-like fragments (PLF), intermediate-mass fragments (IMF), light charged particles (LCP), and neutrons have been performed for the reactions ${ }^{209} \mathrm{Bi}+{ }^{136} \mathrm{Xe}$ and ${ }^{197} \mathrm{Au}+{ }^{208} \mathrm{~Pb}$ at the lower boundary of the Fermi energy regime. The former experiment involved $4 \pi$ detectors for both neutrons and LCP's. The measurements provide compelling evidence for very simple reaction dynamics dominated by collective degrees of freedom. Invariant emission patterns for neutrons and LCP's indicate a binary, dissipative first reaction stage, followed by the decay of the hot reaction products, for essentially the entire range of impact parameters. The multiplicity of evaporated neutrons is found to be a good measure of the dissipated kinetic energy and can be employed as a sensitive indicator of the collision impact parameter. Non-evaporative light particles and IMFs are also observed in the two reactions, but with small multiplicities and most distinctly in peripheral collisions. IMFs detected in peripheral ${ }^{209} \mathrm{Bi} \gamma-136 \mathrm{Xe}$ collisions are possibly due to non-adiabatic neck rupture or squeeze-out. Experimental correlations between particle multiplicity and massive-fragment energy, deflection angle, and Z-distribution suggest the presence of transport phenomena induced by multi-nucleon exchange processes. In both reactions, the initially available kinetic energy is observed to be damped only partially.
\end{abstract}

\section{Introduction}

The study of reactions between complex nuclei at bombarding energies per nucleon of several tens of $\mathrm{MeV}$, the Fermi energy regime, has attracted considerable experimental and theoretical interest over the past decade. This activity reflects the exciting prospects of producing nuclear systems at high temperatures [Lev87, Sur87, 89] and of observing reaction dynamics significantly different from interaction processes known from either much lower or much higher bombarding energies. In this transitional energy regime, one expects an evolution of the reaction mechanism from dynamics dominated by mean-field phenomena to processes characteristic of a growing importance of two-body nucleonnucleon collisions [Gre86,Ayi87,Ber88]. In addition, at nucleus-nucleus approach speeds comparable to the nucleonic Fermi velocities, non-adiabatic effects in the effective conservative and dissipative nuclear interactions may be encountered [Sch84, Nör86]. Qualitatively new reaction phenomena predicted [Ber88,Bon85,Bar90,Gro86,87,93, Fri88,90,Lop89,90] for the Fermi energy domain include an instantaneous multi-fragmentation or vaporization of a hot, expanding system created in an energetic collision of two heavy nuclei. The occurrence of such phenomena that have been associated with a nuclear liquid-gas phase transition is expected to depend dramatically on the magnitude of the nuclear temperatures generated in heavy-ion collisions. 
These prospects have motivated [Gue89,Gal89] an intense search for methods to produce hot nuclei with temperatures near the stability limit and to observe the multi-fragmentation of such nuclei. In fact, observations of copious production of IMF's in some of the reactions have already been interpreted tentatively as a signature of the formation of a composite nuclear system undergoing a critical disassembly process [Bow91]. Yet, inspite of considerable efforts, a consistent understanding of the intermediate-energy heavy-ion reaction mechani sm is still lacking. This could partially be due to the limited range of projectiles that have been used in previous experimental studies [Gre86,Gel87,Bor90], which had to rely mostly on projectiles not heavier than ${ }^{40} \mathrm{Ar}$. Reactions induced by relatively light projectiles exhibit a rich phenomenology depending drastically on the impact parameter, already at low bombarding energies. Their complexity could even increase at higher energies, due to the appearance of fragmentation-type [Gol74, Fri83] or participant-spectator reactions [Day86, Bon87, Gra88]. Recently, however, beams of very heavy ions have become available, enabling one to study very heavy systems for which the reaction mechanism should develop smoothly with impact parameter. For such systems, albeit at lower energies, it is experimentally possible [Sch84] to reconstruct the time evolution or impact-parameter dependence of transfer-induced transport processes leading to a gradual heating of the reaction partners. Here, the reaction phenomena correlate strongly with the amount of kinetic energy dissipated in collision. There is mounting evidence [Gue89, Gal89,Sch92] for a similar correlation at higher energies, when the dissipated energy is measured in terms of the multiplicity of emitted light particles, notably of neutrons.

The above considerations have motivated exclusive experiments on the very heavy systems ${ }^{209} \mathrm{Bi}+{ }^{136} \mathrm{Xe}$ and ${ }^{197} \mathrm{Au}+{ }^{208} \mathrm{~Pb}$ representing attempts to gain a new access to the reaction mechanism at intermediate energies and to establish some of its salient features at the lower boundary of the Fermi energy regime. Specifically, the experiments set out to search for correlations between reaction phenomena and the amount of dissipated kinetic energy or related experimental observables, which had been found very instructive at lower bombarding energies. In the following, the experiments will be described briefly and a survey will be given over the most interesting results.

\section{Experimental Methods}

Experiments were performed for the reactions ${ }^{209} \mathrm{Bi}+{ }^{136} \mathrm{Xe}$ and ${ }^{197} \mathrm{Au}+{ }^{208} \mathrm{~Pb}$ at the MSU/NSCL and GANIL accelerator facilities, delivering ${ }^{136} \mathrm{Xe}$ and ${ }^{208} \mathrm{~Pb}$ beams of 28.2 and $29 \mathrm{MeV}$ per nucleon, respectively. In both cases, charged products ranging from light charged particles to massive, projectile-like fragments were measured in coincidence with neutrons. In the ${ }^{209} \mathrm{Bi}+{ }^{136} \mathrm{Xe}$ experiment, the first of its kind, all particles were measured with essentially $4 \pi$ coverage. The corresponding setup is schematically depicted in Fig. 1 showing the Rochester $4 \pi$ neutron multiplicity meter (NMM) [Bal93] with its tank and internal scattering chamber partially cut open to expose the St. Louis $4 \pi$ DWARF Ball/Wall phoswich multi-detector array [Sar88,Nor87] placed inside the chamber. The detectors, meant to measure mainly LCP's and IMF's were shielded from electrons and elastically scattered projectiles by metallic foils inducing lower detection thresholds of 
$1 \mathrm{MeV}$ and 2-3 MeV per nucleon, for Wall and Ball detectors, respectively. In the present experiment, one of the five segments of the Wall was removed to provide space for three large-area, solid-state detector telescopes covering the forward angles in the range from $|\theta| \approx 3^{\circ}$ to $26^{\circ}$. The $\mathrm{NMM}$ is filled with $\approx 900 l$ of Gd-loaded liquid scintillator with a dual function as neutron moderator and generator of scintillation light following the delayed neutron capture by the Gd component. The NMM provides both a prompt-

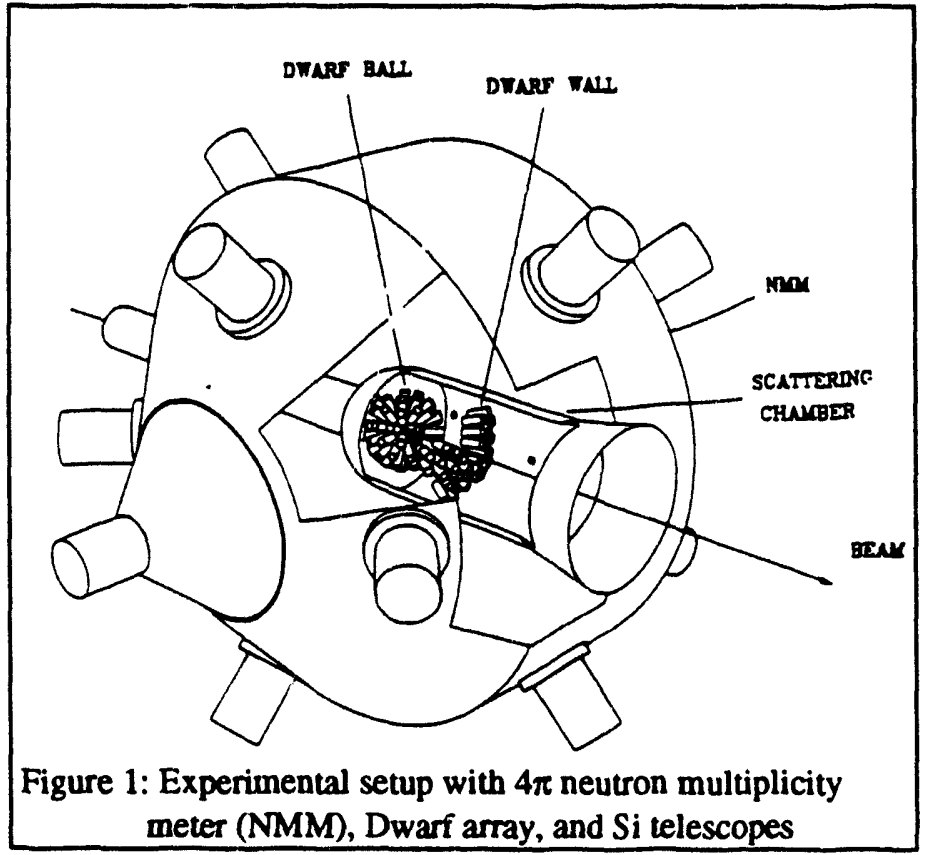

simulation code DENIS [Que89]. response signal, a measure of the total kinetic energy of all neutrons from a reaction event, and a sequence of delayed signals, one for every neutron capture. Hence, total neutron kinetic energy and multiplicity are measurable event by event. The NMM is mainly sensitive (typical efficiency $\varepsilon=0.6-0.8$ ) to neutrons emitted from slowmoving emitters such as the heavy, target-like (TLF) fragments, while the detection efficiency for neutrons for fastmoving PLF's is significantly smaller, as calculated with a modified version of the

The ${ }^{197} \mathrm{Au}+{ }^{208} \mathrm{~Pb}$ experiment [Que93], employed a setup with five solid-state detector telescopes for the measurement of charged products in the angular range from $|\theta|=6.5^{\circ}$ to $141.5^{\circ}$, three large-area Si-strip detectors $\left(|\theta|=28^{\circ}-81^{\circ}\right)$ for measuring kinematical PLF-TLF coincidences, and 21 single NE213 (BC502) liquid-scintillator, neutron timeof-flight (TOF) counters. Thin fast-plastic scintillation counters were placed in front of the forward neutron detectors, in order to identify and veto fast light charged particles having penetrated the thin walls of the scattering chamber. The start signal for the TOF measurement was derived from the (GANIL) accelerator RF signal, and the discrimination between $\gamma$-rays and neutrons was accomplished by an analysis of the experimental pulse shapes and of correlations between TOF and scintillator light output.

\section{Dissipative Dynamics of ${ }^{209} \mathrm{Bi}+{ }^{136} \mathrm{Xe}$ and ${ }^{197} \mathrm{Au}+{ }^{208} \mathrm{~Pb}$ Collisions}

\subsection{Massive-Fragment Distributions}

Very detailed data on massive, projectile-like fragments were obtained for both the reactions ${ }^{209} \mathrm{Bi}+{ }^{136} \mathrm{Xe}$ and ${ }^{197} \mathrm{Au}+208 \mathrm{~Pb}$. In the former case, it was a position-sensitive silicon detector telescope that enabled the measurement of a partial elastic-scattering angular distribution in the range $3^{\circ} \leq \theta \leq 5.6^{\circ}$, just missing the grazing angle of $7.2^{\circ}$, but providing a 
calibration of the measured yield by normalization to the Rutherford cross section. The experimental average yield obtained with this telescope is plotted in Fig. 2 vs fragment laboratory energy $E$ and atomic number $Z$. A direct integration of the fragment yield for $Z Z 20$ results in a cross section of approximately $\sigma=5.7 \mathrm{~b}$, which exhausts the total reaction cross section of $\sigma=5.9 \mathrm{~b}$ predicted by systematics. For such a comparison to be meaningful, double-counting of events leading to massive fragments has to be avoided. This requirement is trivially fulfilled for fragments with $\mathrm{Z}$ significantly larger than half that of the projectile, and is approximately met for $Z \geq 20$. Therefore, the distribution of relatively massive products seen in Fig. 2 is representative of the overall reaction and not just of rather peripheral collisions, as might have been suspected. The fragment Z-E correla-

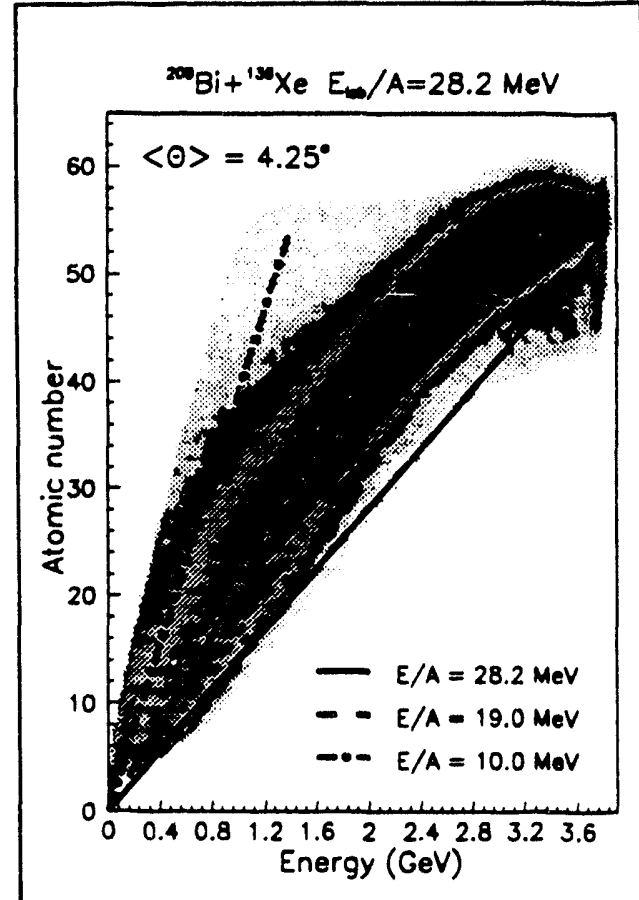

Figure 2: Experimental charged-product distribution for the reaction ${ }^{209} \mathrm{Bi}+{ }^{136} \mathrm{Xe}$ at $\langle\theta\rangle=4.3^{\circ}$, plotted vs. $Z$ and energy. tion shown in Fig. 2 has a shape typical also for the ${ }^{197} \mathrm{Au}+{ }^{208} \mathrm{~Pb}$ system and for other heavy-ion reactions in this energy regime. A well-defined ridge of cross section is seen to extend from the elastic-scattering peak $(Z=54, E=3.8 \mathrm{GeV})$. For $Z \geq 35$, the intensity along the ridge first decreases with decreasing $Z$ and $E$, while for $Z \leq 35$, it remains relatively constant, down to very small $Z$ values. For both reactions, an almost linear character is observed for the correlation between $\mathrm{Z}$ and $E$ such as shown in Fig.2, suggesting that the fragments all have velocities of the order of the beam velocity and are, hence, remnants of the projectile. Similar distributions for other systems [Luc87, Gra88] in this energy regime have in the past been interpreted in terms of participantspectator [Gol74,Gra88] or incomplete-fusion models [Gue89]. Such models predict cold PLF's to be emitted at forward angles essentially with beam velocities. For orientation, straight lines are included in Fig. 2 corresponding to three different constant values of $\mathrm{E} / \mathrm{A}$ or fragment velocity. Obviously, the average PLF energy per nu-

cleon is approximately equal to $19 \mathrm{MeV}$ (dashed line), the average velocity is significantly lower than the beam velocity (solid line), a fact indicating considerable energy dissipation. The degree of energy dissipation, or energy loss $\left(E_{\text {loss }}\right)$, is, hence, reflected in both final fragment laboratory energy and atomic number. It is also remarkable that this average velocity is higher than that (dot-dashed line) corresponding to a full damping of the initial kinetic energy and that there are actually very few events that could be associated with such low, fully damped energies.

For the ${ }^{197} \mathrm{Au}+{ }^{208} \mathrm{~Pb}$ reaction, a Z-E correlation qualitatively very similar to that of Fig. 2 is measured. However, while the distribution of fragments from the ${ }^{209} \mathrm{Bi}+{ }^{136} \mathrm{Xe}$ reaction shows no evidence of sequential PLF fission, such events are evident in the corresponding distribution for the reaction ${ }^{197} \mathrm{Au}+{ }^{208} \mathrm{~Pb}$. In the $\mathrm{Pb}$-induced reaction, the somewhat 
steeper slope of the Z-E correlation in the quasi-elastic region is understood to be due to the fact that a relatively high fissility of the majority of the heavier PLF's leads to a depletion of the fragment $Z$ distribution, leaving only few PLF's in the low- $Z$ wings intact which, like the Xe-like fragments, do not undergo sequential fission. Simulation calculations based on the nucleon-exchange model (NEM)[Ran82, Døs85] discussed further below show that the characteristic Z-E correlations for both the ${ }^{209} \mathrm{Bi}+{ }^{136} \mathrm{Xe}$ and ${ }^{197} \mathrm{Au}+{ }^{208} \mathrm{~Pb}$ reactions can be understood quantitatively in terms of the decay of the primary PLF's that have been produced in binary dissipative reactions with a broad distribution in $\mathrm{Z}$ and $\mathrm{A}$. In the case of the latter reaction, a kinematical coincidence measurement of PLF-TLF pairs was possible which proved the validity of the assumed dissipative reaction scenario, at least for total kinetic energy losses up to $\mathrm{E}_{\text {loss }}=350 \mathrm{MeV}$. This scenario is also consistent with the emission patterns of light particles discussed below.

The study of correlations between emission angle of reaction products, notably of PLF's, and dissipated energy or energy loss $\left(E_{\text {loss }}\right)$ has played an important role in the study of collision dynamics at lower bombarding energies [Sch84], where it has lead to the introduction of the notion of classical friction forces and of other transport phenomena. Since the production cross section of massive fragments in the heavy-ion reactions under study appears to be very large, an inspection of such correlations is important. This is possible for the reaction ${ }^{209} \mathrm{Bi}+{ }^{136} \mathrm{Xe}$, for which a position-sensitive Si-detector telescope, centered at the angle of $\theta_{\mathrm{lab}}=4.25^{\circ}$, was available. The experimental correlation

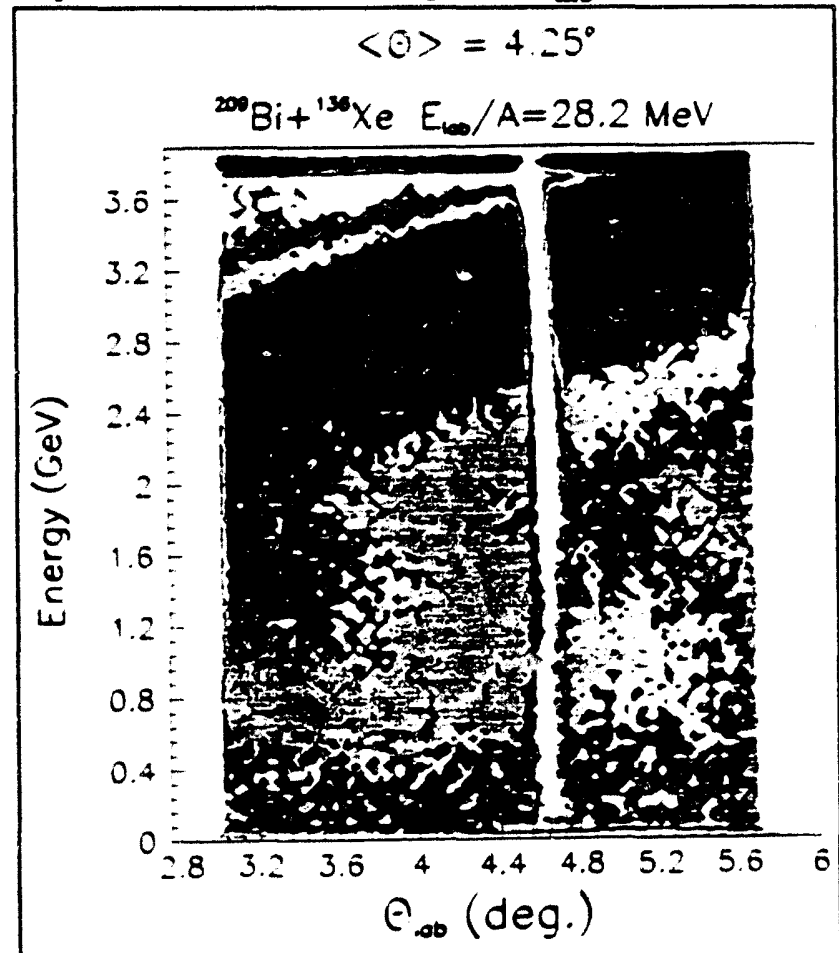

Figure 3: Contour diagram of the correlation between laboratory energy and reaction angle for massive fragments from the reaction ${ }^{209} \mathrm{Bi}+{ }^{136} \mathrm{Xe}$. between emission angle, $\theta_{\text {lab }}$, and laboratory PLF kinetic energy, also termed "Wiiczyñski plot", is shown in Fig.3, for this reaction, in form of a contour plot. Here, the fragment energy, uncorrected for evaporation effects, can be considered a measure of the dissipated energy. The vertical gap appearing on the plot is the "shadow" of a mask used for position calibration. The band of high yield at the top of the figure represents the elastic-scattering ridge extending up to the grazing angle $\left(\theta_{\mathrm{g}} \approx 70\right)$, which is beyond the scope of the figure. Emerging from this ridge, i.e., from the quasi-elastic peak at the largest angles, one observes a ridge of reaction yield extending forward in angle with decreasing fragment energy or increasing energy loss. This latter ridge extends obviously further forward than the cutoff at an angle of $3^{\circ}$, imposed on the data by the finite 
acceptance of the telescope. In addition to this forward-going ridge, there is an additional ridge at low energies, extending backward in angle and fading in intensity, as the PLF energy decreases further. One is tempted to interpret the dependence of the yield on PLF energy and deflection angle observed in Fig. 3 as a reflection of a continuous classical deflection function $\Theta(b)$, where the impact parameter $b$ is represented by the final fragment energy. The behavior of this deflection function, observed here for the first time in the Fermi energy regime, is surprisingly reminiscent of the phenomenon of dissipative orbiting observed for asymmetric reactions at lower bombarding energies [Sch84], where the intermediate dinuclear system, subjected to dissipative interaction forces, rotates about its center of gravity to forward angles and even to (negative) angles on the other side of the beam.

The physical foundations of such a very simple reaction mechanism, in which the identities of projectile and target nuclei are preserved to a large extent, are understood in the regime of low energies. However, such a mechanism is generally not expected in the Fermi energy regime, where excitation energies should be sufficient to effect an almost complete disassembly of the system, for a large range of impact parameters. Nevertheless, it is interesting to note that simulation calculations [Sch87] for the reaction ${ }^{209} \mathrm{Bi}+{ }^{136} \mathrm{Xe}$ at $\mathrm{E} / \mathrm{A}=28.2 \mathrm{MeV}$, based on the standard one-body, nucleon-exchange model NEM [Ran82, Døs85] do, in fact, predict qualitatively a behavior as observed in Fig.3. These calculations use liquid-drop forces with proximity corrections in the adiabatic limit, where a neck is formed between the reaction fragments. An alternative explanation of the appearance of a deflection function, such as shown in Fig.3, is based on "nuclear rainbow" scattering, where the deflection function $\Theta(b)$ exhibits a minimum at relatively forward angles. Such a behavior is also predicted by the NEM, but in the limit of nuclear forces based on a frozen-density approximation of the interaction partners. In both types of simulation calculations, however, complete damping of the available kinetic energy is predicted to occur with significant cross section, quite in contrast to the experimental observation of incomplete damping.

\subsection{Distributions of Light Charged Particles and Neutrons}

A rather complete characterization of collision events in terms of the multiplicities and energies of the associated light particles was achieved for the reaction ${ }^{209}{ }^{\mathrm{Bi}+}{ }^{136} \mathrm{Xe}$, due to the availability of $4 \pi$ detectors for both charged particles and neutrons. Experimental results for the correlation between the observed multiplicities of light charged particles and neutrons are displayed in Fig. 4 in form of a contour diagram. The multiplicities are not corrected for the finite detection probabilities (efficiency and effective solid angles), which are in the present case quite similar for charged particles and neutrons. The correlation shown in this figure provides a survey over the development of particle emission processes with increasing amount of dissipated kinetic energy, since the total particle multiplicity obviously provides some kind of scale for the dissipated energy. One concludes from the characteristic correlation shown in Fig. 4 that the first $300-400 \mathrm{MeV}$ of excitation energy, generated most likely in a peripheral collision, lead mainly to the emission of neutrons, 
while the most probable charged-particle multiplicity is zero in this region. This implies that charged-particle emission is hindered by significant Coulomb barriers. For more dissipative collisions, charged particles compete more successfully with neutrons, and the multiplicity correlation of Fig. 4 exhibits a relatively constant slope. This distribution exhibits a broad bump at $\left\langle\mathrm{m}_{\mathrm{n}}\right\rangle=26$ and $\left\langle\mathrm{m}_{\mathrm{LCP}}\right\rangle=15$, which is associated with highly dissipative, more central collisions. The actual average multiplicities are estimated to be of the order of $\left\langle\mathrm{m}_{\mathrm{n}}>\approx 58-60\right.$ and $\left\langle\mathrm{m}_{\mathrm{LCP}}>\approx 20\right.$.

From the shape of the multiplicity correlation depicted in Fig.4, one concludes that the

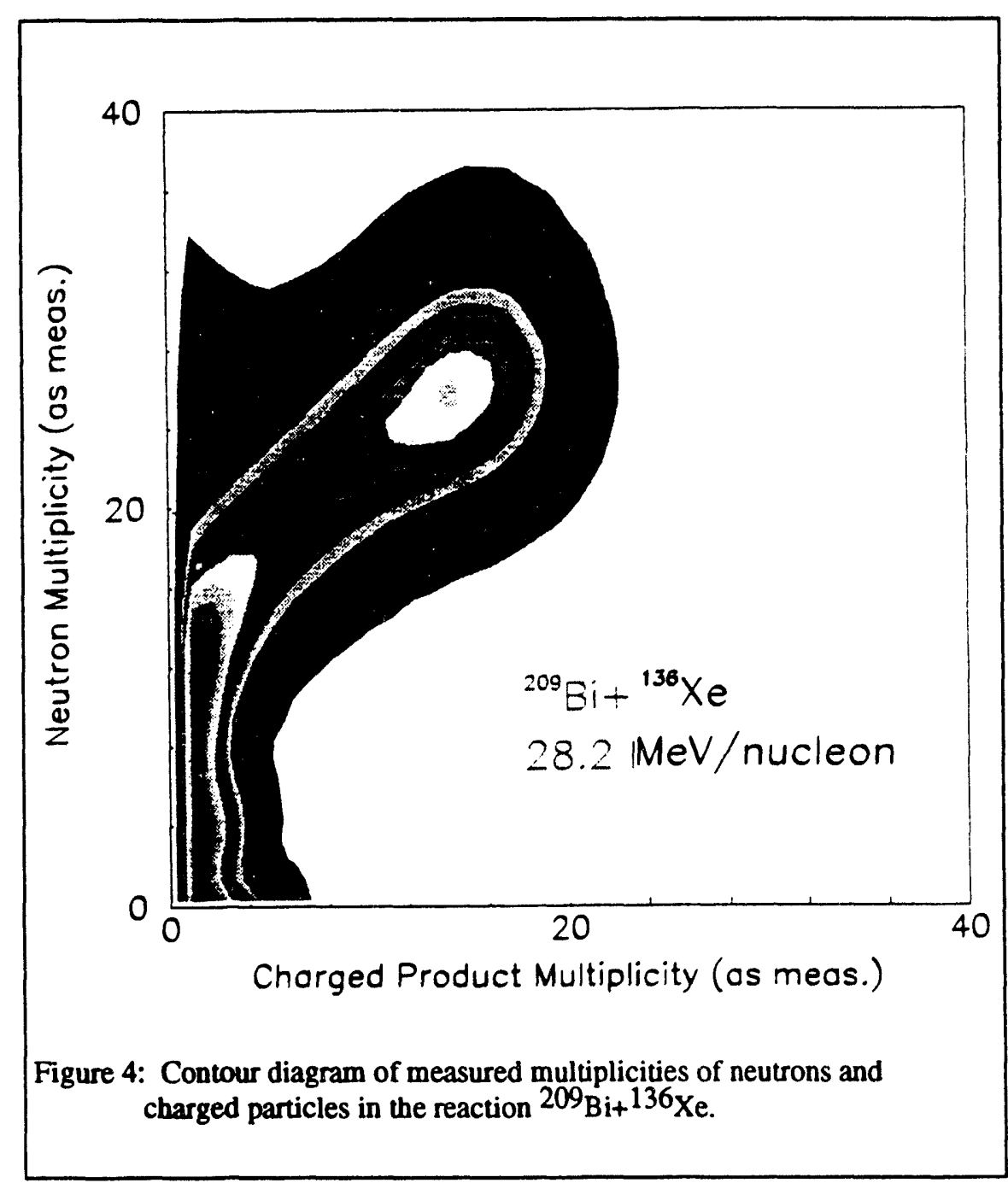
neutron multiplicity is a rather sensitive indicator of the dissipated energy, over the whole range of possible energy losses. The charged-particle multiplicity, on the other hand, provides a sufficiently accurate measure of the dissipated energy for intermediate to high degrees of dissipation. To the extent that the dissipated energy reflects the collision impact parameter, the particle multiplicity observables can be exploited for impact parameter selection of reaction events. The suitability of the neutron multiplicity for this latter purpose has been demonstrated [Gue89,Gal89,Pia91, Lot92], at least for relatively heavy reaction systems. 
More direct and detailed information about a reaction can in principle be inferred from the

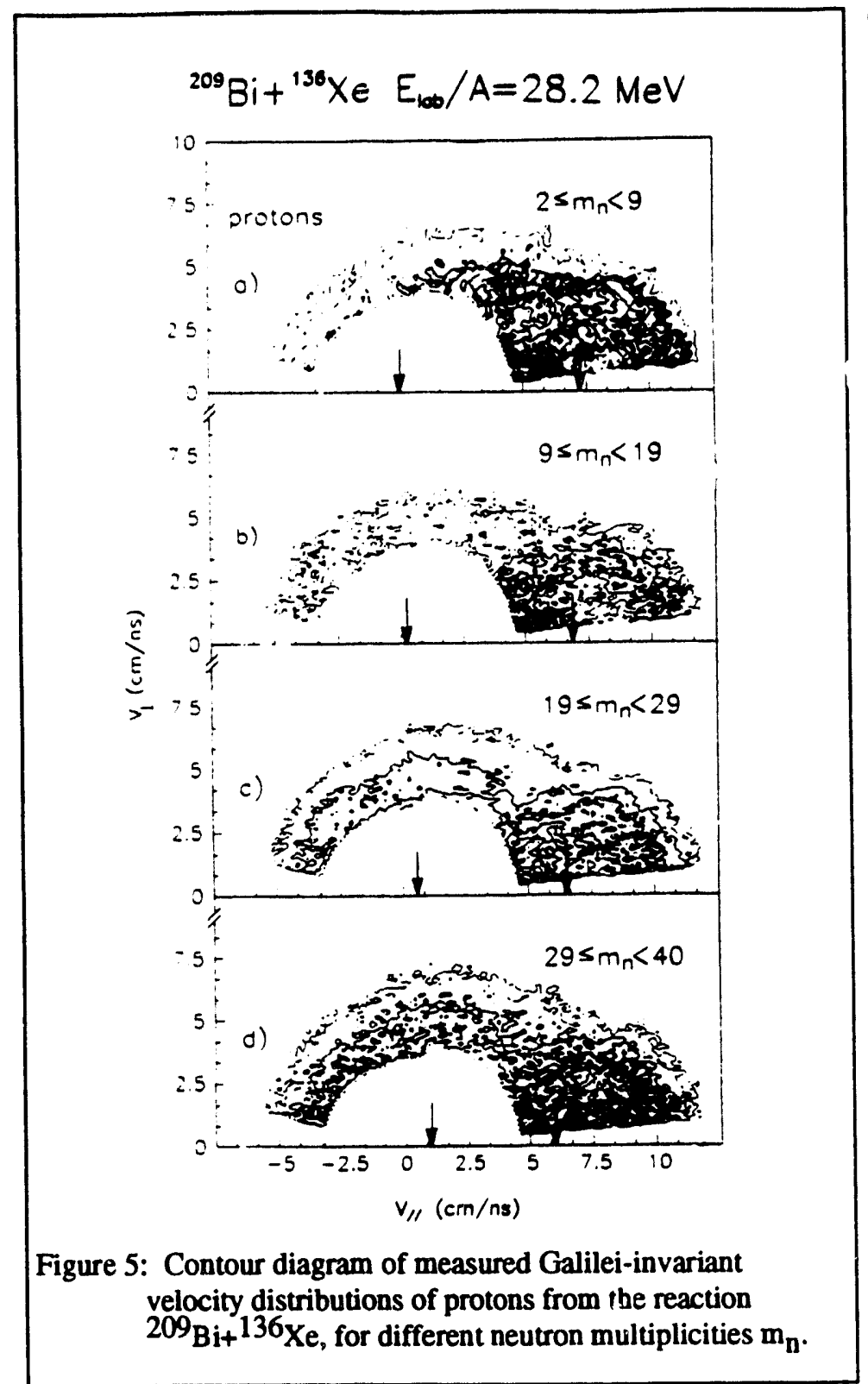
emission patterns of the associated light particles. The Galilei-invariant velocity distributions of such particles depend in a characteristic way on the kinematical properties and the temperature of the emitters, if such emitters are welldefined and few in number. In Fig.5, linear two-dimensional maps of the Galilei-invariant cross section $d^{2} \sigma / v_{\perp} d v_{\perp} d v_{\|}$of protons measured [Lot92] for the reaction ${ }^{209} \mathrm{Bi}+{ }^{136} \mathrm{Xe}$ are plotted versus the particle velocity components parallel $\left(v_{\|}\right)$ and perpendicular $\left(v_{\perp}\right)$ to the beam direction. These protons were measured in coincidence with neutrons in the multiplicity bin indicated in each panel. The set of neutron multiplicity bins covers essentially the total reaction cross section. The finite multiplicity resolutions of the detectors are always smaller than the widths of the multiplicity bins.

As seen from this figure, the proton emission patterns are bimodal, consisting of two distinct semi-circular ridges with centers indicated by the two arrows. The radii of the cross section ridges corresponding to the most probable velocities coincide quite accurately with the Coulomb velocities of protons emitted from a fast Xe-like fragment and its slow Bi-like reaction partner, identifying sequential emission from primary PLF and TLF as the major production mechanism of these particles. In fact, the PLF and TLF velocities indicated by the arrows in Fig. 5 have been calculated by relating the integrated particle multiplicities to the dissipated energy, implying the damped reaction scenario [Sch84]. The invariant proton distributions shown in Fig.5 are representative of those for other light charged particles measured in the same experiment. All patterns are observed to evolve in a smooth and systematic fashion with increasing degree of dissipation, measured either by the multiplicity of neutrons or by that of charged particles. The distributions retain their 
bimodal character even for collisions associated with the highest degrees of dissipation measured, for example those accompanied by the emission of six IMF's $\left(Z_{\mathrm{MF}} \geq 4\right)$ ! The

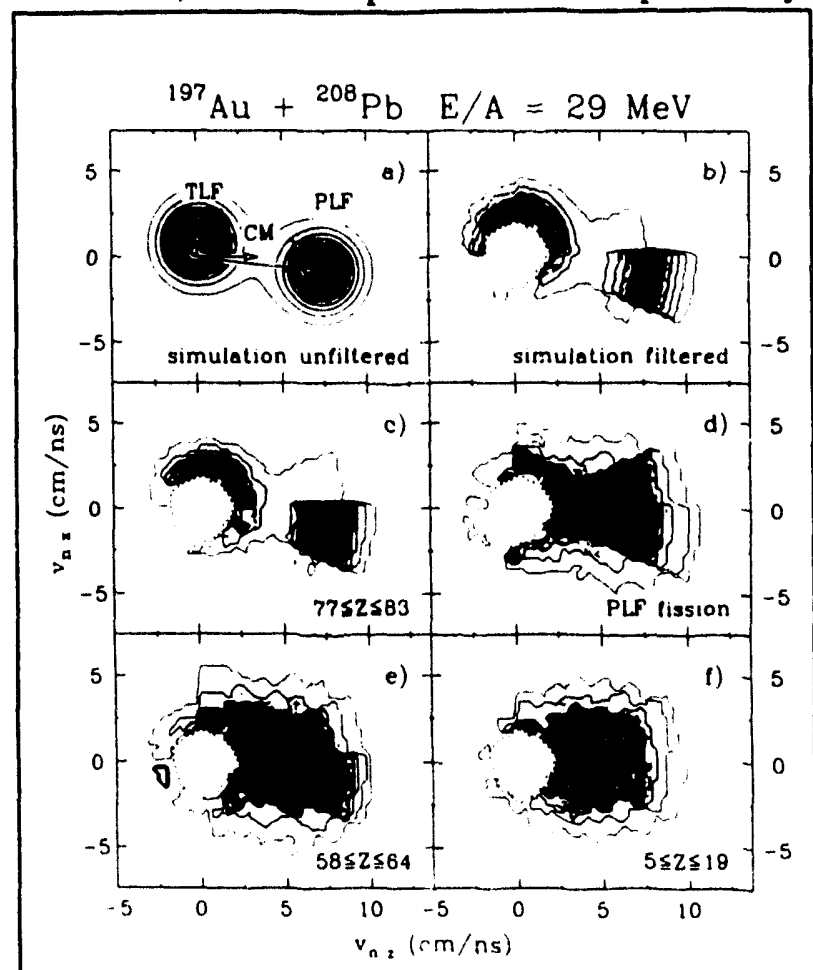

Figure 6: Contour diagrams of simulated $(a, b)$ and measured (c-f) Galilei-invariant velocity distributions of neutrons from the reaction ${ }^{197} \mathrm{Au}+{ }^{208} \mathrm{~Pb}$, in coincidence with fragments of different $Z$-values. present data demonstrate firstly and very clearly a bimodal character of the reaction leading to two massive fragments in a first reaction step, followed by a sequential decay of these primary fragments. While such a mechanism is expected for peripheral collisions, its prevalence for essentially all impact parameters comes as a great surprise and challenges the current theoretical understanding of heavy-ion reactions. Secondly, the data suggest that the maximum dissipated energy does not exceed $50 \%-60 \%$ of the total kinetic energy available in the entrance channel. This conclusion, too, seems difficult to reconcile with widely held views of intermediate-energy heavy-ion reactions. The results of the ${ }^{197} \mathrm{Au}+{ }^{208} \mathrm{~Pb}$ experiment corroborate the findings for the system ${ }^{209} \mathrm{Bi}+{ }^{136} \mathrm{Xe}$ discussed above. This is illustrated in Fig.6, exhibiting Galilei-invariant velocity distributions of neutrons from the reaction ${ }^{197} \mathrm{Au}+{ }^{208} \mathrm{~Pb}$ at $\mathrm{E} / \mathrm{A}=29 \mathrm{MeV}$, plotted versus two velocity components. The neutrons

were measured in coincidence with different types of charged reaction products detected at $\theta=-6.5^{\circ}$. These "trigger" fragments represent different degrees of dissipation, and the panels c) - f) are arranged according to increasing energy loss, and presumably decreasing impact parameter. The uppermost panels represent a theoretical distribution for a peripheral collision with an energy loss of only $50 \mathrm{MeV}$ (a) and the same distribution folded with the relatively coarse detector response (b). As can qualitatively be inferred from Fig. 6 and is shown by numerical fits of the data with distributions calculated from models assuming several representative moving sources, the neutron emission patterns of Fig. 6 are compatible with sequential evaporation from primary PLF and TLF, i.e., again suggest binary collision dynamics. As the Z-value of the trigger fragment is decreased, i.e., with increasing degree of dissipation, the integrated neutron multiplicity increases, and the emission patterns become more compact. However, even for events triggered by IMF's (Fig.6f) are the neutron patterns incompatible with the single emitter source expected from theoretical models, but can be reproduced rather well assuming two (PLF and TLF) sources [Que93]. 
The simulation calculations that are compared to the data refer to source velocities, as well as to energy spectra and total multiplicities of the neutrons assumed to be emitted isotropically from these sources. For example, highly dissipative collisions selected by trigger fragments with $58 \leqq \mathrm{Z} \leqq 83$ (cf. Fig.6e) are found to be associated with average multiplicities of neutrons from PLF and TLF of $\left\langle\mathrm{m}_{\mathrm{PLF}}\right\rangle=\left\langle\mathrm{m}_{\mathrm{TLF}}\right\rangle=25 \pm 4$, spectral slope parameters (effective source temperatures) of $\tau_{\mathrm{PLF}}=\tau_{\mathrm{TLF}}=(3.7 \pm 0.4) \mathrm{MeV}$, and with source velocities indicating a kinetic-energy loss of $E_{\text {loss }}=(1.5 \pm 0.2) \mathrm{GeV}$. Some of the results of such model comparisons are summarized in Fig.7, where the total integrated neutron multiplicity $m_{\text {tot }}$ is plotted $v s$. the energy loss deduced from the source velocities, assuming binary kinematics. Although the uncertainties in the $\mathrm{E}_{\text {loss }}$ variable are quite large for the higher energy losses, the correlation in Fig.7 is well-defined, providing an independent proof of the sensitivity of the neutron multiplicity observable as an indicator of the energy dissipated in a collision. The solid curve in Fig.7 represents the prediction of the statistical-model code EVAP [Nic92] for the correlation $\mathrm{m}_{\text {tot }}\left(\mathrm{E}_{\text {loss }}\right)$, for representative ${ }^{197} \mathrm{Au}$ and ${ }^{208} \mathrm{~Pb}$ fragments.

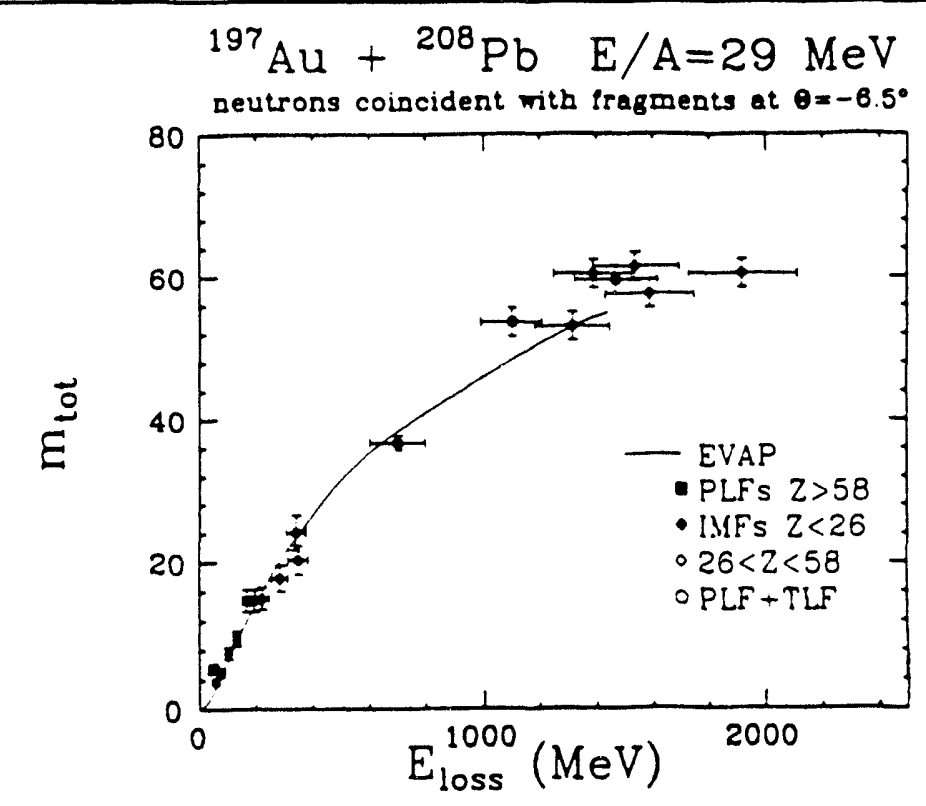

Figure 7: Total multiplicity of neutrons from the reaction

${ }^{197} \mathrm{Au}+{ }^{208} \mathrm{~Pb}$ vs. energy loss derived from source velocities. The solid line represents evaporation calculations.
Consequently, the parameters deduced from the experimental data form an internally consistent set of observables describing the decay of hot primary fragments, as ascertained by the above statisticalmodel calculations. As indicated in the legend of Fig.7, events triggered by IMF's are found to be associated with the highest neutron multiplicities, as well as with the highest value of $E_{\text {loss }}=(1.9 \pm 0.2)$ $\mathrm{GeV}$ measured in the experiment. They also show the highest effective source temperatures.

However, this amount of dissipated energy is significantly smaller than the kinetic energy of $\mathrm{E}_{\mathrm{cm}}-\mathrm{V}_{\mathrm{Coul}}=2.5 \mathrm{GeV}$ available above the entrance-channel barrier. These observations of incomplete damping are, hence, made consistently for the two different heavy-ion systems studied here.

\subsection{Non-Evaporative Particle Emission}

As discussed above, both the ${ }^{209} \mathrm{Bi}+{ }^{136} \mathrm{Xe}$ and the ${ }^{197} \mathrm{Au}+{ }^{208} \mathrm{~Pb}$ reaction have an essentially binary character with only two massive primary fragments in the entrance channel. 
This binary character remains unaffected by the relatively small multiplicities of nonevaporative particles that have also been observed in the present experiments, most distinctly for peripheral collisions. For such quasi-elastic collisions, the evaporative particle components due to the sequential decay of excited primary fragments are relatively weak. For example, non-evaporative neutrons from the reaction ${ }^{197} \mathrm{Au}+{ }^{208} \mathrm{~Pb}$ were detected [Que93] in coincidence with PLF's in the range of atomic numbers $77 \leqq Z_{\mathrm{PLF}} \leqq 83$. The corresponding emission pattern can be adequately described [Hil84, Wil89] in terms of an effective hypothetical source, emitting particles isotropically and traveling with approximately half the beam velocity. From numerical fits, an average multiplicity of $m_{n e}=1.0 \pm$ 0.4 and a spectral slope parameter of $\tau_{n e}=(7 \pm 1) \mathrm{MeV}$ are found for these nonevaporative neutrons. The values of both parameters are somewhat smaller than predicted by systematics [Hil84, Wil89] based on data obtained for lighter systems and lower bombarding energies. There is some evidence in the ${ }^{197} \mathrm{Au}+{ }^{208} \mathrm{~Pb}$ data that the values of both $m_{n e}$ and $\tau_{n e}$ increase with increasing degree of dissipation, although experimental uncertainties are significant [Que93]. Calculations with a Fermi-jet model [Ran87] are relatively successful in the description of the dependence of the multiplicity $\mathrm{m}_{\mathrm{ne}}$ on the value of $E_{\text {loss, }}$, but fail to reproduce the shape of the non-evaporative neutron emission pattern. Investigations of charged particles have not progressed far enough yet to produce definite conclusions, but non-evaporative emission processes for charged particles are expected to occur with characteristics similar to those of the corresponding neutron component.

Of particular interest are the IMF production mechanisms studied in some detail for the reaction ${ }^{209} \mathrm{Bi}+{ }^{136} \mathrm{Xe}$. IMF's are, in this work, defined to encompass light charged products with $4 \leq Z_{\mathrm{IMF}}$, measured with the detectors of the DWARF array. Since the emission of IMF's is characteristic of energetic and highly dissipative heavy-ion collisions [Mor88], but is not observed in reactions at lower bombarding energies, an understanding of the corresponding production mechanism is crucial for a comprehensive evaluation of intermediate-energy reaction mechanisms. IMF's are produced in intermediate-energy heavy-ion collisions with multiplicities exceeding those consistent with statistical evaporation from hot, massive composites, and with angular distributions that are anisotropic in the rest frames of these fragments. The average IMF multiplicity in the reaction ${ }^{209} \mathrm{Bi}+{ }^{136} \mathrm{Xe}$ is generally much smaller than unity but increases monotonically with increasing degree of dissipation, as measured either by the neutron multiplicity or atomic number or number of a PLF measured in coincidence, with a maximum of $\mathrm{m}_{\mathrm{IMF}}=3.4$, for the most highly dissipative collisions measured. Fig. 8 provides a comparison between the Galilei-invariant velocity diagrams of $\alpha$-particles (left) and IMF's with $4 \leq Z_{\mathrm{IMF}} \leq 6$ (right), accumulated for the same binary collision events and a sequence of bins in $\mathrm{Z}$-value $\left(\mathrm{Z}_{\text {coinc }}\right)$ of the coincident massive trigger fragments. There is obviously a striking difference between these two types of distributions. Whereas the $\alpha$-particle distributions show the familiar Coulomb ring patterns, due to sequential emission from PLF and TLF (cf. Fig.5), the IMF pattern are dominated by a peak associated with velocities intermediate between those of PLF and TLF. These patterns suggest that, in quasi-elastic collisions selected by large values of $Z_{\text {coinc }}$, IMF's are produced dominantly with low velocities in the contact region between the interacting PLF and TLF. Their 
energy spectra are well reproduced by Maxwellians in the center-of-mass system. For a representative intermediate degree of dissipation or $E_{\text {loss }}\left(12 \leq m_{n} \leq 14\right)$, a Maxwellian fit yielded an effective Coulomb barrier of only $5 \mathrm{MeV}$ but a spectral slope parameter of 30 $\mathrm{MeV}$.

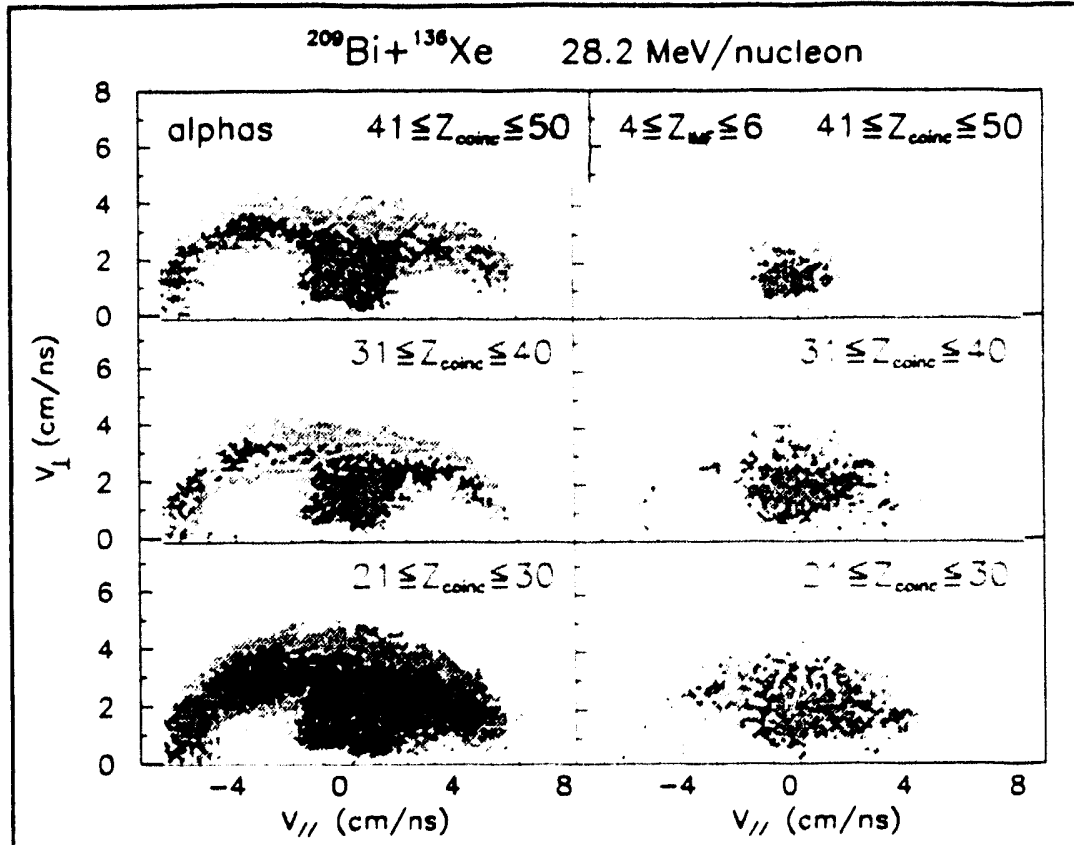

Figure 8: Contour diagram of measured Galilei-invariant velocity distributions of $\alpha$ - particles (left) and IMFs (right) from the reaction ${ }^{209} \mathrm{Bi}+{ }^{136} \mathrm{Xe}$, in coincidence with massive fragments in the Z-bins indicated.
It can also be argued that these IMF's are relatively cold. Sequential emission of charged particles by the IMF's would otherwise be expected to destroy the well-defined Coulomb patterns observed in Fig.8. As seen also from this figure, for more dissipative collisions selected by lower $Z_{\text {coinc }^{-1 / a l u e s, ~}}$ Coulomb rings begin to develop also in the IMF patterns, indicative of increasing probability for sequential IMF emission

from PLF and TLF. Although different emission mechanisms contribute to the IMF intensity at different angles, the $\mathrm{Z}_{\mathrm{IMF}}$ - yield distributions are all of an exponential shape with a slope parameter increasing with increasing $E_{\text {loss }}$ or neutron multiplicity.

There are several scenarios that could, in principle, contribute to the non-evaporative IMF component. However, ordinary pre-equilibrium processes or the decay of a fireball produced in the geometrical overlap region of PLF and TLF seem incompatible with the data and can be excluded from the list of possible IMF production mechanisms. At this stage, it appears likely that these IMF's are produced in the rupture of a neck that has been formed transiently between the fragments but is unable to follow adiabatically the collective motion of the reseparating reaction fragments.

\subsection{Reassembly of Reaction Primaries}

The rather complete coverage of the light particles emitted in coincidence with massive PLF's that was achieved in the ${ }^{209} \mathrm{Bi}+{ }^{136} \mathrm{Xe}$ experiment, provides the unique opportunity to reconstruct at least the atomic-number distributions of the corresponding primary fragments, as a function of dissipated energy. In the analysis, a first estimate of the primary PLF atomic number was calculated by adding to that of the identified secondary PLF the total charge of LCP's attributed to that PLF, scaled with the corresponding detection 
efficiency. The effect of IMF emission has not been incorporated in the analysis yet. Average efficiencies were calculated from the invariant emission patterns of the LCP's.

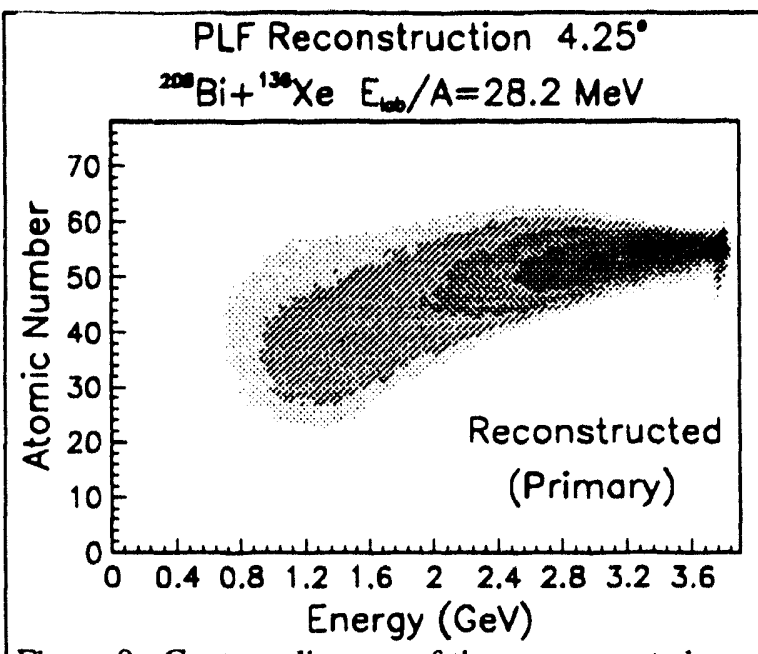

Figure 9: Contour diagiom of the reconstructed primary $\mathrm{Z}$ distribution of PLF's from the reaction ${ }^{209} \mathrm{Bi}+{ }^{136} \mathrm{Xe}$ measured at $\theta \approx 4^{\circ}$.

Their finite values introduce an instrumental broadening of the deduced distributions. Fig. 9 shows a contour diagram of the PLF yield plotted vs. the reconstructed primary $\mathrm{Z}$ value and the laboratory PLF energy. One observes from this figure that the primary $\mathrm{Z}$ distribution remains approximately centered at the $\mathrm{Z}$ value of the projectile $\left(Z_{\mathrm{PLF}}=54\right)$, while it broadens significantly with decreasing fragment energy or increasing energy loss. At the lower PLF energies, or higher degrees of dissipation, the average $Z$ value appears to decrease by several units. This may, at least partially, reflect the emission of IMF's, which has not yet been accounted for in the analysis. The approximately equal probabilities for

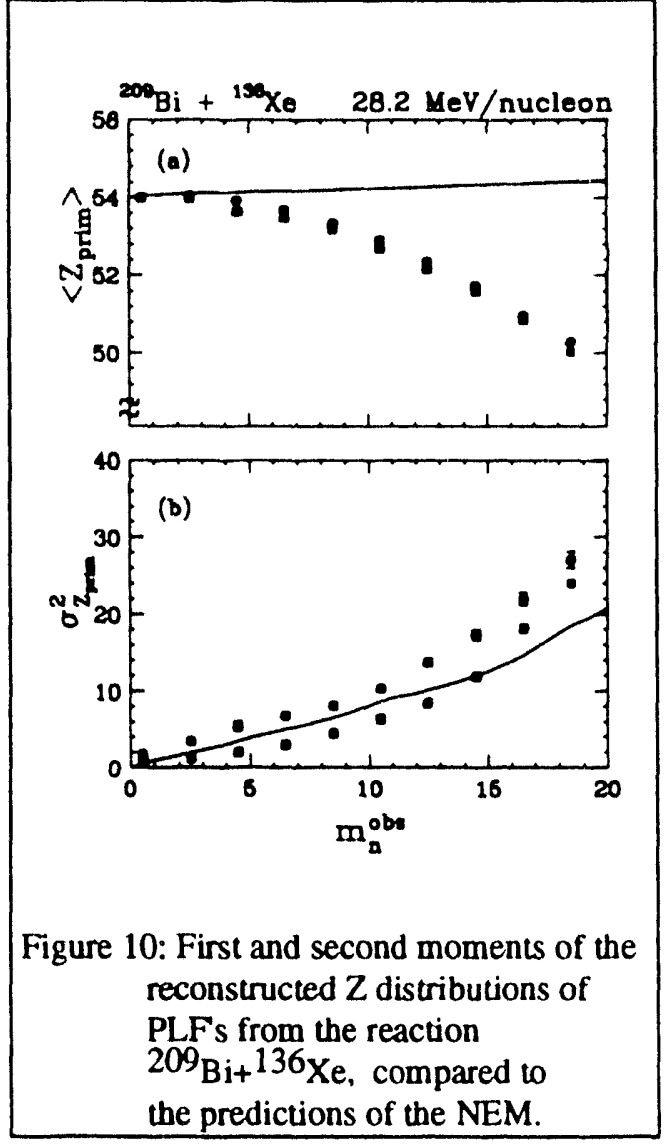
multi-proton stripping and pickup by the projectile eliminates participant-spectator or incomplete-fusion models as viable reaction mechanisms. In contrast, this feature is a wellknown property of damped nuclear reactions at much lower energies.

A numerical analysis has been performed of the reconstructed, almost Gaussian primary $\mathrm{Z}$ distributions. In Fig. 10, the values of the first and second moments, $\langle\mathrm{Z}\rangle$ and $\sigma_{\mathrm{Z}}{ }^{2}$, respectively, resulting from this analysis are plotted vs. the observed neutron multiplicity $m_{n}$. The solid circles represent the numerically evaluated moments, while the squares correspond to moments derived from Gaussian fits to the data. These experimental results are compared to the predictions (curves) of the NEM transport model referred to previously. Considering the uncertainties associated with the present, tentative analysis, one has to consider the agreement between data and calculations remarkable. The relatively large discrepancies occurring for the first moments 
can perhaps be understood to be partially due to the emission of IMF's at high energy losses, which is not accounted for in the model calculations.

It is rather interesting to note that the correlation between charge variance and dissipated energy illustrated in Fig.10 continues the trends observed at much lower energies [Sch84]. These trends have been adequately described by one-body transport calculations similar to those reported here. This interesting observation could imply that the two-body effects, predicted [Ayi87] to lead to fragment $Z$ distributions of much increased widths, are not as important at Fermi energies as previously thought.

\section{Conclusions}

The present study of the two very heavy systems ${ }^{209} \mathrm{Bi}+{ }^{136} \mathrm{Xe}$ and ${ }^{197} \mathrm{Au}+{ }^{208} \mathrm{~Pb}$ has revealed several remarkable features of the reaction mechanism operating at bombarding energies of the order of the nucleonic Fermi energies. Although these energies are sufficient to lead to the disintegration of a substantial part of the system, projectile and target nuclei are able to largely preserve their identities in a first, essentially binary reaction step. The dynamics of the reaction is dominated by collective effects leading to a dissipative-orbiting or double-rainbow deflection function. The probability for a complete damping of the initially available kinetic energy of relative motion was observed to be very small.

For the reaction ${ }^{209} \mathrm{Bi}+{ }^{136} \mathrm{Xe}$, it was possible to reconstruct the $\mathrm{Z}$ distribution of the primary PLFs. It appears that multi-nucleon exchange processes still occur at these bombarding energies, leading to fragment $Z$ distributions that continue the trends observed at lower energies.

It was found that neutrons and light charged particles are dominantly emitted sequentially from the accelerated fragments. The multiplicities of these particles, particularly the neutron multiplicity, provide good estimates of the excitation energy acquired by the fragments and, hence, give an indication of the impact parameter. There are only relatively weak components of neutrons and charged particles emitted in non-evaporative processes. Such a component is also observed for MMF's, whose kinematical properties suggest non-adiabatical neck rupture as a possible production mechanism. Alltogether, the experimental results reported here on two very heavy systems provide a consistent experimental picture of a mechanism governed by very simple collective dynamics which may originate from microscopic processes similar but probably not identical to those established at lower energies. These results present important challenges to the current theoretical understanding of intermediate-energy reaction mechanisms.

\section{Acknowledgment}

The data presented in this particle are due to the efforts of several collaborations. The team of Ref. [Que93] generated the data on the ${ }^{197} \mathrm{Au}+{ }^{208} \mathrm{~Pb}$ reaction, while results for the ${ }^{209} \mathrm{Bi}+{ }^{136} \mathrm{Xe}$ results were obtained by the collaboration signing for Ref. [Lot92,Bal93]. 
This work was supported by the U.S. Department of Energy under grant number DE-FG02-88ER40414.

\section{References}

Ayi 87 S. Ayik, Phys. Rev. C35, 2086(1987)

Bal 93 S.P. Baldwin, PhD Thesis, University of Rochester, 1992

Ba193 S.P. Baldwin et al., Nucl. Instr. Meth. Phys. Res., to be published

Bar90 H.W. Barz et al., Phys. Let. B244, 161(1990)

Ber 88 G.F. Bertsch and S. Das Gupta, Phys. Rep. 160, 189 (1988), and references

Bon 85J.P. Bondorf et al., Nucl. Phys. A443, 321 (1985); ibid A444,460(1985)

Bon 87 A. Bonasera et al., Nucl. Phys. A463, 653 (1987)

Bor 90 B. Borderie et al., Ann. Phys. (Fr.) 15, 287 (1990)

Bow91 D.R.Bowman et al., Phys. Rev. Lett. 67, 1527(1991)

Day 86R. Dayras et al., Nucl. Phys. A460, 299 (1986).

Døs85 T. Dössing et al., Nucl. Phys. A327, 490 (1979); ibid. A433, 280 (1985)

Fri 83 W.A. Friedman et al., Phys. Rev. C27, 569 (1983); ibid. C28, 16 (1983)

Fri 88 W.A. Friedman, Phys. Rev. Let. 60, 2125 (1988)

Fri 90 W.A. Friedman, Phys. Rev. C42, 667 (1990)

Gal 89 J. Galin, Proc. Symp. Nucl. Dyn. Nucl. Disassembly, J.B. Natowitz, editor, (World Scientific, Singapore, 1989), p.320.

Gel 87 C.K. Gelbke and D.H. Boal, Progr. Part. Nucl. Phys. 19, 33 (1987), and references therein

Gol 74 A.S. Goldhaber, Phys. Lett. 53B, 306 (1974)

Gra 88 O. Granier et al., Nucl. Phys. A481, 109 (1988)

Gre 86 C. Gregoire and B. Tamain, Ann. Phys. Fr. 11, 323 (1986) and references cited therein.

Gro 86 D.H.E. Gross et al., Phys. Rev. Lett. 56, 1544 (1986)

Gro 87 D.H.E. Gross et al., Nucl. Phys. A461, 641 (1987); A461, 668 (1987)

Gro93 D.H.E. Gross, Prog. Part. Nucl. Phys. 30, 155(1993), and references therein

Gue 85D. Guerreau, Nucl. Phys. A442, 37C (1985).

Gue 89D. Guerreau, in Nuclear Matter and Heavy-Ion Collisions M. Soyer, H. Flocard, B. Tamain, and M. Porneuf, editors, (Plenum Publ. Corp., 1989), p. 187; and references cited therein.

Hil 84 D. Hilscher et al., Proc. Worksh. Coinc. Part. Emission Cont. States, Bad Honnef 1984 (P. David, editor), World Scientific, Singapore, 1984, p. 268

Lev 87 S. Levit and P. Bonche, Nucl. Phys. A462, 109 (1987)

Lop89 J.A. Lopez and J. Randrup, Nucl. Phys A503, 183(1989)

Lop90 J.A. Lopez and J. Randrup, Nucl. Phys A512, 345(1990) 
Lot 92 B. Lott et al., Phys. Rev. Lett. 68, 3141 (1992)

Luc87 R. Lucas et al., Nucl. Phys. A464, 172(1987)

Mor 88L.G. Moretto and G.J. Wozniak, Progr. Part. Nucl. Phys. 21, 401 (1988).

Nic92 N.G. Nicolis et al., Comp. Phys. Comm., in press

Nör86 W. Nörenberg, New Vistas in Nuclear Physics, P.J. Brussard(Editor), Plenum Press New York (1986), p.91

Nor87 E. Norbeck et al., Nucl. Inst. Meth. A262, 546(1987)

Que 89B. Quednau and D. Pade, Report DOE/ER/40414-2 (W.U.

Schröder and J.R. Huizenga, edt.), p.170 (1989)

Que93 B.M. Quednau, PhD Thesis Ruhr-Universität Bochum, Germany, 1993; and B.M. Quednau et al., Phys. Lett. B, in press.

Ran 82 J. Randrup, Nucl. Phys. A383, 468 (1982)

Ran 87 J. Randrup et al., Nucl. Phys. A474, 219 (1987)

Sar88 D.G. Sarantites et al., Nucl. Inst. Meth. Phys Res. A264, 319 (1988)

Sch84 W.U. Schröder and J.R. Huizenga, Treatise on Heavy-Ion Science, (D.A. Bromley, Editor), Plenum Press (New York and London,1984), Vol.2, pp.113-726; and references cited therein

Sch87 W.U. Schröder et al., Nucl. Sci. Res. Conf. Ser.(Harwood) Vol. 11, 255 (1987)

Sch 92 W.U. Schröder, Nucl. Phys. A538, 439c (1992)

Sur 87 E. Suraud, Nucl. Phys. A462, 109 (1987)

Sur 89 E. Suraud, Proc. Symp. Nucl. Dyn. Nucl. Disassembly, J.B. Natowitz, editor, (World Scientific, Singapore, 1989), p. 464.

Wil 89 J.L. Wile et al., Phys. Rev. Lett. 63, 2551(1989) 


\title{
Correlations Between Neutrons and Charged Products from the Dissipative Reaction \\ ${ }^{197} \mathrm{Au}+{ }^{208} \mathrm{~Pb}$ at $\mathrm{E} / \mathrm{A}=29 \mathrm{MeV}$
}

\author{
B.M. Quednau, S.P. Baldwin, B. Lott, W. U. Schröder, B.M. Szabo, J. Tõke \\ Department of Chemistry and Nuclear Structure Research Laboratory \\ University of Rochester, Rochester, New York 14627, USA
}

D. Hilscher, U. Jahnke, H. Rossner

Hahn-Meitner-Institut Berlin, 1000 Berlin 39, Germany

\author{
S. Bresson, J. Galin, D. Guerreau, M. Morjean \\ IN2P3-CNRS and DSM-CEA \\ GANIL, F-14021 Caen Cedex, France.
}

\begin{abstract}
Exclusive Measurements of neutrons in coincidence with charged reaction products bave been performed for the reaction ${ }^{197} \mathrm{Au}+{ }^{208} \mathrm{~Pb}$ at $\mathrm{E} / \mathrm{A}=29 \mathrm{MeV}$. These products range from light charged particles (LCP) to massive projectile-like fragments (PLF). For peripheral collisions associated with energy losses of Eloss $\leq 300 \mathrm{MeV}$, a kinematical coincidence measurement demonstrates the existence of a binary, dissipative reaction mechanism. The measured PLF atomic-number distributions are found to be consistent with primary distributions that have widths increasing with increasing energy loss, but reflect only the low-7, wings of the distributions which are not affected by fission. The reaction mechanism for more penetrating, and central collisions is inferred from the invariant velocity distributions of neutrons in coincidence with PLF's of relatively low atomic numbers and with intermediate-mass fragments (IMF). Consistently for all types of events, including those accompanied by IMF's, these neutron emission patterns are found to be dominantly bimodal and are understood to result from sequential emission of neutrons from fully accelerated primary PLF's and their target-like (TLF) reaction partners. A relatively weak non-equilibrium neutron component is also observed. Primary fragment distributions and average velocities, as well as the multiplicities and the slope parameters of the energy spectra of sequentially emitted neutrons, are consistent with a picture of a dissipative reaction mechanism based on the exchange of nucleons. However, even in highly dissipative IMF accompanied collisions, on the average only up to $75 \%$ of the kinetic energy available in the entrance channel can be dissipated.
\end{abstract}




\section{Introduction}

The phenomenology of heavy-ion nuclear reactions at bombarding energies of a few tens of $\mathrm{MeV}$ per nucleon, the Fermi energy regime, has been under investigation now for over one decade [Gre86, Gel87, Ber88, Gue89, Bor90]. However, progress that has been made so far in characterizing intermediate-energy reactions has not generated a consensus in the views of the underlying microscopic reaction mechanisms. While the low-energy reaction dynamics [Sch84] is comparatively well explained in terms of the damped (or dissipative) reaction mechanism, modeled quantitatively in the microscopic/macroscopic one-body nucleon exchange model NEM [Ran82,87, Døs85], a number of different reaction mechanisms still contend for the corresponding position at the higher Fermi energies.

The currently significant experimental and theoretical interest in intermediate-energy heavyion reactions is generated largely by the expectation of qualitatively new reaction phenomena [Ber88, Bon85, Bar90, Gro86,87,93, Fri88,90, Lop89,90] at nucleus-nucleus approach velocities comparable with the nucleonic Fermi velocities. In this transitional bombarding energy regime, the dominance of the mean field should give way to a growing importance of two-body nucleon-nucleon collisions [Gre86, Ayi87, Ber88] and higher nucleonic correlations; and non-adiabatic effects in the effective conservative and dissipative nuclear interactions may be encountered [Sch84, Nör86]. More dramatic reaction phenomena such as nuclear vaporization or instantaneous multi-fragmentation could occur, provided that critically high excitation energies per nucleon of $\varepsilon^{*} \sim 5-6 \mathrm{MeV}$ [Lev87, Sur87,89] are reached in the interacting nuclear systems. The occurrence of such phenomena has been associated with a nuclear liquid-gas phase transition. Alternatively, and not less interestingly, nuclear multi-fragmentation has been related [Mor92] to a macroscopic, Rayleigh-type instability of hot nuclear matter, recently discussed [Bro83,89,90] in the context of nuclear fission and low-energy heavy-ion reactions.

Experimentally, however, no direct demonstration of instantaneous multi-fragmentation or vaporization, concurrent with the macroscopic collision, is available yet, nor is it even clear what experimental reaction phenomena could constitute unambiguous signals for these processes. Nevertheless, the observation of a nearly complete disassembly of nuclear reaction fragments in individual intermediate-energy heavy-ion reactions, or the copious production of intermediate-mass fragments (IMF's) with probabilities exceeding those 
expected from statistical-model calculations [Mor88, Kim92], have already been taken, if tentatively, as indications of such new reaction modes.

In this situation, the most promising strategy for a discovery of new reaction modes seems to be a systematic tracing of the reaction phenomenology from the comparatively wellknown domain of bombarding energies of a few MeV per nucleon into the Fermi energy regime. Such systematic studies are still lacking. Some of the experimental difficulties and ambiguities are undoubtedly related to the nature of reaction systems studied thus far experimentally. With only few exceptions, these past studies have considered reactions induced by ${ }^{40} \mathrm{Ar}$ or lighter projectiles. These reactions exhibit a rich phenomenology already at relatively low bombarding energies, including the processes of quasi-elastic, damped, incomplete fusion/massive transfer, complete fusion-evaporation and fusionfission reactions. At the higher energies of interest here, additional processes of fragmentation [Gol74, Fri83] or participant-spectator reactions [Day86, Bon87, Gra88] could further complicate the picture. Reactions between very heavy nuclei, on the other hand, are at lower bombarding energies associated [Sch84] with a simpler and more uniform (damped) reaction mechanisms that develops smoothly with impact parameter. This feature makes reactions between very heavy ions most amenable to mechanistic studies at the lower boundary of the Fermi energy regime. Since, at the lower energies, reaction theory has succeeded in providing a quantitative explanation of many aspects of heavy-ion collisions in terms of a multi-nucleon exchange model [Ran82,87, Døs85], it is deemed instructive to subject an extrapolation of this model into the Fermi energy regime to experimental test.

The present work forms part of a larger systematic investigation of a very heavy system, in which as many aspects as possible of the symmetric and neutron-rich, very-heavy-ion reaction ${ }^{197} \mathrm{Au}+{ }^{208} \mathrm{~Pb}$ at $\mathrm{E} / \mathrm{A}=29 \mathrm{MeV}$ are studied in different experiments. For an orientation, the pertinent parameters of this reaction are presented in Table 1. Earlier and preliminary accounts of several of these experiments have already been given elsewhere[Pia90, Bre92, Que91,92,93,93a]. The specific goal of the present study was to measure neutrons emitted in the reaction ${ }^{197} \mathrm{Au}+208 \mathrm{~Pb}$ at $\mathrm{E} / \mathrm{A}=29 \mathrm{MeV}$, in coincidence with the entire spectrum of charged reaction products, and to provide an interpretation of the results in terms of the dominant reaction mechanism. The following Section II discusses the experimental methods, while Section III gives a brief overview of theoretical simulation calculations performed for the ${ }^{197} \mathrm{Au}+{ }^{208} \mathrm{~Pb}$ reaction. The experimental data are presented in Section IV. This section also offers an interpretation of the data in terms 
of a surprisingly simple dissipative reaction mechanisms that bears many similarities to lowenergy dynamics but exhibits also features that distinguish it from the damped interactions well-established at the lower energies. Some comparisons between data and model calculations will also be given in Section IV. A summary is presented in Section V.

\section{Experimental Methods and Analysis}

\section{II.1. Experimental Setup and Calibrations}

The experimental measurements were performed at the GANIL accelerator facility. This accelerator provided a pulsed ${ }^{208} \mathrm{~Pb}$ beam of $\mathrm{E} / \mathrm{A}=29 \mathrm{MeV}$. The pulse period was typically $128 \mathrm{~ns}$, with a pulse width of the order of $1 \mathrm{~ns}$. This heam was used to bombard a self-supporting ${ }^{197} \mathrm{Au}$ target of $2.1 \mathrm{mg} / \mathrm{cm}^{2}$ thickness, placed in the center of a thinwalled scattering chamber. A schematic illustration of the experimental setup is given in Fig. II.1. It shows several solid-state detector telescopes, large-area position-sensitive Sistrip detectors, and 23 neutron detectors outside the chamber, some of them equipped with additional "anticoincidence" counters. Conventional electronics were used throughout for electronic signal processing, along with the GANIL VAX-based data acquisition system.

Three $\Delta \mathrm{E}-\Delta \mathrm{E}-\mathrm{E}$ telescopes were placed at forward angles to measure the energies $\mathrm{E}$ and atomic numbers $\mathrm{Z}$ of massive, projectile-like fragments (PLF), two other, three- or fourelement telescopes were employed to detect light charged particles (LCP) and intermediate-mass fragments (IMF) with $\mathrm{Z}_{\mathrm{IMF}} \leq 3$. Table 2 lists the angles and physical parameters of these telescopes. To protect the telescopes from $\delta$-electrons produced by the beam in the target, $1.8-\mathrm{mg} / \mathrm{cm}^{2}$ and $0.4-\mathrm{mg} / \mathrm{cm}^{2}$ thick $\mathrm{Al}$ absorber foils were placed in front of the PLF and LCP telescopes, respectively. Foils and, in particular, the relatively thick first $\triangle E$ element of each PLF telescope resulted in energy thresholds of identified massive fragments of the order of $E_{\mathrm{th}} / \mathrm{A}=5-8 \mathrm{MeV}$.

Large-area $\left(4 \times 6 \mathrm{~cm}^{2}\right)$ Si-strip detectors of $300 \mu \mathrm{m}$ nominal thickness were used in a kinematical-coincidence measurement of PLF-TLF pairs, involving the telescope PLF1 $\left(\theta=-6.5^{\circ}\right)$, and covered, in steps of $5^{\circ}$ and with only small gaps, the angular range of 
$25^{\circ} \leq \theta \leq 81^{\circ}$. These detectors were protected from $\delta$-electrons by $0.2-\mathrm{mg} / \mathrm{cm}^{2}$ thick $\mathrm{Al}$ absorber foils. Timing signals were derived from two out of the seven strip elements of each of these detectors, in addition to pulse-height information, in order to determine the time of flight (TOF) and, hence, the velocity of measured products. Energy and time resolutions obtained with these detectors were sufficient to separate slow, heavy TLF recoils from light charged particles.

The pulse height responses of the above solid-state detectors were calibrated using standard radioactive $\alpha$ and ${ }^{252} \mathrm{Cf}$ fission sources, in combination with linear pulse generators. In addition, elastically scattered projectiles provided valuable calibration references for telescopes PLF1 and PLF2. Corrections for energy loss in the absorber foils and for the pulse-height defect were applied using available systematics [Mou78, Zie85]. Typical energy resolutions for elastically scattered projectiles obtained with the PLF telescopes were of the order of 5\%. The PLF telescopes had energy (loss) resolutions sufficient to provide $Z$ identification for massive fragments with atomic numbers of $Z \leq 30$, 35, and 24, for PLF1, PLF2, and PLF3, respectively. As an example, the correlation $\Delta \mathrm{E}_{1}-\Delta \mathrm{E}_{2}$ between the pulse heights measured with the first two elements of telescope PLF3 is displayed in Fig. II.2, demonstrating the resolution in colomic number achieved with this telescope. Electronic thresholds in the first $\triangle \mathrm{E}$ detectors of the PLF telescopes prevented charged products with $\mathrm{Z} \leq 4$ to be identified with these detectors.

Twenty-one neutron detectors were used to measure the neutron time-of-flight (TOF) spectra. The detectors consisted of cylindrical vessels of diameters between 12.7 and 25.4 $\mathrm{cm}$ and lengths of 3.9 to $10.2 \mathrm{~cm}$, filled with NE213 or BC502 organic liquid scintillator, coupled to Philips XP204I photomultipliers. They were placed at distances between 1 and $2 \mathrm{~m}$ from the target, in slane with the PLF telescopes (cf. Fig.II.1), and covered the angular range of $-165^{\circ} \leq 9_{\mathfrak{n}} \leq+163^{\circ}$. Two detectors, positioned out of this plane, were used for consistency checks but did not provide data of high accuracy. As seen in Fig. II.1, the forward neutron detectors were preceded by thin (3-6 mm) NEl02 plastic-scintillator "anticoincidence" counters, forming scintillation counter telescopes providing identification of charged particles that penetrated the thin-walled scattering chamber and entered the neutron detectors.

The neutron TOF was measured relative to the accelerator RF signal. The typical time resolution achieved, of the order of $\Delta \mathrm{t} \approx 1 \mathrm{~ns}$, was dominated by the width and jitter of the ${ }^{208} \mathrm{~Pb}$ beam pulses relative to the RF signals and corresponded to energy resolutions of 
the order of $\Delta E_{n} \approx 2 \mathrm{MeV}$ for neutron energies of $E_{n}=10 \mathrm{MeV}$ and $\Delta E_{n} \approx 8 \mathrm{MeV}$ at $E_{n}=$ $30 \mathrm{MeV}$. The light output spectra of the NE213 detectors were calibrated with standard $\gamma$ ray sources. Electronic detection thresholds were set at equivalent proton recoil energies of approximately $0.5 \mathrm{MeV}$, for detectors at backward angles, and at approximately 1 $\mathrm{MeV}$, for detectors located at forward angles. The thresholds used in the off-line data analysis were significantly higher, between 0.7 and $2.8 \mathrm{MeV}$.

In Fig.II.3, a raw TOF spectrum containing both neutrons and $\gamma$-rays is shown on logarithmic scale by a thin histogram, as obtained with a neutron detector at $\theta_{\mathrm{n}} \approx 37.8^{\circ}$. A "prompt" TOF peak, associated with $\gamma$-rays in the neutron detector, is seen on the left, while the broader distribution on the right following the gap corresponds to neutrons. Also indicated on this figure are the largest physical TOF corresponding to the threshold applied to the scintillator light output signal, and the range used to determine the background in the spectrum. The heavy histogram in Fig.II.3 indicates mainly the contribution of neutrons to the total TOF spectrum, although this spectrum shows contamination by a small portion of the $\gamma$-ray events.

Discrimination between neutrons and $\gamma$-rays was accomplished mainly with dedicated electronic pulse-shape discrimination modules (PD6 or PD7, developed by the HMI-Berlin Electronics Division). For particular events, use was also made of the characteristic correlation between TOF and light output of the liquid scintillator, and the information gathered with the fast-plastic anticoincidence counters. Examples of such correlations between pulse-shape signal $\Delta S$, TOF signal, and light output are shown in Fig. II.4 in form of scatter plots, for one particular neutron/anticoincidence counter telescope. Parts a) and c) show the correlations as measured in anticoincidence with the thin plastic counter, i.e., they represent the neutron (NE213) detector response to neutrons or $\gamma$-rays, which do not trigger the anticoincidence counter. In Fig. II.4a), one observes clearly three intense ridges. The Compton electron ridge (e) is caused by incoming $\gamma$-rays, while the other two ridges represent the response to neutrons. Most events produce elastically scattered protons ( $p$ ), while the ridge labeled " $\alpha$ " provides evidence for the $(n, \alpha)$ reaction with the ${ }^{12} \mathrm{C}$ content of the scintillator. The various branches seen in the panels $b$ ) and $c$ ) correspond to $\mathrm{p}, \mathrm{d}, \mathrm{t}$, and $\alpha$ particles incident on the plastic/NE213 detector telescope.

The TOF spectra measured with the neutron detectors contained background from different sources. The background due to cosmic rays and other sources uncorrelated with the beam is approximately random in time. Its intensity can, therefore, be determined from regions 
in the TOF spectrum inaccessible to reaction events. In addition, there is background caused by beam particles, not in the target but in the general surroundings of the detector setup. This background has, therefore, the same intrinsic time structure as true reaction events and can conceivably introduce spurious structure in the TOF spectra measured relative to a charged-particle trigger. This latter type of background was measured for selected neutron detectors, during the regular experiment, by placing $60-\mathrm{cm}$ long brass and iron absorbers ("shadow bars") in front of these detectors, blocking their direct view of the target.

Following the data-taking runs, a measurement of the well-known [Bow62, Bud88] ${ }^{252} \mathrm{Cf}$ fission neutron spectrum was conducted, in order to be able to verify the functionality of the TOF spectrometer and the data analysis procedures. In this experiment, a ${ }^{252} \mathrm{Cf}$ spontaneous-fission source was placed in target position, facing a solid-state detector detecting fission fragments and providing start signals for the neutron TOF measurement. Different geometries of fission detector and ${ }^{252} \mathrm{Cf}$ source were employed in separate measurements, in order to study the effects of scattering and absorption of the ${ }^{252} \mathrm{Cf}$ fission neutrons by the various solid-state detector telescopes used in the actual ${ }^{197} \mathrm{Au}+{ }^{208} \mathrm{~Pb}$ experiment.

\section{II.2. Data Analysis}

The kinetic energies of charged products detected in the solid-sate detector telescopes were determined from the calibration measurements described in the preceding section. The theoretical maximum energy deposits ("punch-through energies") of charged products of given atomic and mass numbers in a given detector, calculated according to Ziegler [Zie85], were used to provide additional calibration points for A- and Z-identified fragments. The $\triangle \mathrm{E}-\mathrm{E}$ data of these, relatively light products were also used to determine, in an iterative fashion, the actual thicknesses of active and passive layers of the solid-state detector telescopes, by comparing the experimental with the theoretical $\Delta \mathrm{E}-\mathrm{E}$ curves. It turned out that the first two of the three elements of the most forward telescope PLF1 were not fully depleted during the data taking runs, while similar passive layers were not observed for the other telescopes. Although undesirable, such experimental insufficiencies had no serious consequence for the interpretation of data. In the analysis, the kinetic energies of charged products were determined from telescope PLF1, PLF2, and PLF2 data with typical overall accuracies of the order of $5 \%$. 
For fragments with atomic numbers of $Z>30$ (telescope PLF1) or $Z>35$ (telescope PLF2), where the $\triangle E-E$ data measured in these telescopes did not provide unit $Z$ resolution, the conversion to atomic number was based on an interpolation between the last resolved $\Delta \mathrm{E}$ $E$ branch and the slit-scattering branch caused by scattering of the projectile ( $\mathrm{Z}=82)$ in the detector apertures. For the PLF3 telescope, showing no elastic-scattering events and, consequently, no slit-scattering branch, the identification of atomic numbers larger than $\mathrm{Z}=\mathbf{2 4}$ was based on an extrapolation. In all cases, a relative scaling law $\mathrm{Z}(\Delta \mathrm{E}, \mathrm{E})$ was employed as described by Ziegler [Zie85]. Corrections for energy losses in the target and in the protective foils in front of the telescopes were calculated according to the same scheme. In order to determine the true fragment energies, the measured apparent energies were corrected for the pulse height defect as described by Moulton et al.[Mou78]. All of the final corrections and conversions of the data were performed event by event.

For binary collisions with small to moderate (negative) Q-values, where the PLF's and their associated TLF reaction partners survive the sequential deexcitation process essentially intact, it was possible to perform kinematical PLF-TLF coincidence measurements, involving the telescope PLF1 $\left(\theta_{\mathrm{PLF}}=-6.5^{\circ}\right)$ and the large-area strip detectors. These measurements provided a means to determine an average of the total amount of kinetic energy dissipated or otherwise lost from the relative motion. This determination was based on the average PLF and TLF scattering angles and on the average PLF energy, assuming binary kinematics. An iterative procedure was used to determine the average masses of primary PLF and TLF, as well as the total kinetic energy loss $E_{\text {loss. }}$. Results of this kinematical-coincidence measurement are collected in Fig.II.5, where the coincident PLF yield is plotted $v$ s atomic number $\left(Z_{P L F}\right)$, as a function of the TLF correlation angle $\theta_{\mathrm{TLF}}$. The figure shows that, with decreasing correlation angle $\theta_{\mathrm{TLF}}$, the $Z_{P L F}$ distribution broadens, with an average $Z_{P L F}$ (arrow heads in Fig.II.5) shifting to smaller $Z$ values. Such a behavior is qualitatively expected for a binary reaction. For such reactions, a decreasing correlation angle indicates an increasing energy loss and hotter primary fragments, which decay sequentially, leaving broader distributions of secondaries. Very high energy losses were observed to lead to increasing broadening and eventual disappearance of all PLF-TLF correlations, possibly an effect of the recoil imparted on the fragments by the considerable number of evaporated particles. In addition, the cross section decreases dramatically with increasing energy loss, such that these coincidence measurements were not feasible for energy losses $E_{\text {loss }} \geq 350 \mathrm{MeV}$. A quantitative discussion of the results obtained in this correlation experiment will be given in Section IV. 
As mentioned in the previous section, the neutron TOF spectra was measured relative to the accelerator RF signal. The spectra contained background from random sources, e.g., cosmic rays, and beam-induced background. The background caused by beam particles in the general surroundings of the detector setup was determined by a separate "shadow bar" measurement. It turned out that the beam-related neutron background was also essentially random in time with respect to a charged-particle event measured with one of the detector telescopes. Structure in the total TOF background spectrum was found to be due to a group of $\gamma$-rays, emitted probably following neutron activation reactions in the surroundings. These latter $\gamma$-ray background contributions were effectively suppressed by the neutron- $\gamma$ pulse-shape discrimination circuitry. The intensity of the former, random type of background was determined from regions in the TOF spectrum inaccessible to reaction events, extrapolated to the region of interest in the TOF spectrum, and then subtracted from the spectrum with standard event-by-event methods.

The velocity of neutrons was determined from the measured TOF and the effective length of the flight path of the neutrons from target to the interaction site in the scintillator. Neutrons with relatively small kinetic energies $(1-2 \mathrm{MeV})$ produce an interaction within the first few $\mathrm{cm}$ of scintillator, the neutron mean free path is of the order of $\lambda \approx 3 \mathrm{~cm}$. For high energies, on the other hand, the thicknesses of the scintillators used in the experiment were smaller than the associated neutron mean free path, such that the interactions occur on average in the middle of the scintillator. This energy dependence of the effective flight path was accounted for in the analysis.

The measured neutron intensities were scaled according to the detection efficiencies $\varepsilon_{\mathrm{i}}$ of the neutron counters, which were calculated with the Monte Carlo simulation code by Cecil et al.[Cec79]. The analysis of the ${ }^{252} \mathrm{Cf}$ fission data obtained in the present work yielded neutron energy spectra with shapes that were in good agreement with those reported in the literature [Bud88], indicating that the relative detection probabilities were well accounted for in the simulation calculations[Que92]. In addition, the absolute neutron yields measured with most neutron counters agreed well with the predicted intensities, except for several of the larger neutron counters placed in the forward hemisphere which required overall intensity renormalization factors of the order of 1.5-2.0. The precise origin of these discrepancies, which could be due to an overestimation of effective thresholds on the light output signals, solid angles and/or a variety of other factors, was, unfortunately, never discovered. However, the magnitude of the renormalization factors was confirmed by the apparent left-right asymmetries of the 
intensities of neutrons, measured in the actual experiment in coincidence with a light charged particle detected at a backward angle $\left(\theta_{\mathrm{lcp}} \approx 141.2^{\circ}\right)$ angle, i.e., under trigger conditions that should lead to neutron patterns that are approximately symmetric with respect to the beam direction (cf. Sect.IV).

From the measured number, $n_{j}$, in each TOF interval $i$, the calculated velocity-dependent detection efficiency, $\varepsilon_{\mathrm{i}}$, and the detector solid angle $\Omega$, the average differential neutron multiplicity for a given class of events is calculated as

$$
\frac{d m}{d \Omega}\left(\theta_{n}\right)=\frac{1}{N} \sum_{i=1}^{N} \frac{n_{i}}{\varepsilon_{i} \Omega}
$$

Here, the sum is taken over all events of that class. For TOF intervals corresponding to neutron energies below the detection thresholds, the multiplicities $n_{i} / N \varepsilon_{i} \Omega$ were extrapolated assuming an evaporation-type neutron spectrum discussed further below. In order to obtain a relatively model-independent estimate of the total neutron multiplicity, $\mathrm{m}_{\text {tot }}$, one integrates Eq. II.1 over the solid angle, according to

$$
m_{\text {tot }}=2 \pi \frac{1}{2} \int_{-\pi}^{+\pi} d \theta_{n} \frac{d m}{d \Omega}\left(\theta_{n}\right) \sin \theta_{n} \approx \pi \sum_{n=1}^{21} \frac{1}{2}\left(\left|\theta_{n+1}-\theta_{n}\right|+\left|\theta_{n}-\theta_{n-1}\right|\right) \frac{d m}{d \Omega}\left(\theta_{n}\right) \sin \theta_{n}
$$

In Eq. II.2, the sum is carried out over all in-plane neutron detectors $\mathrm{n}$, and the differential multiplicity is interpolated linearly between two adjacent detector angles $\theta_{\mathrm{n}}$ and $\theta_{\mathrm{n}+1}$. Furthermore, azimuthal isotropy is assumed in performing the integration in Eq. II.2.

For the purpose of a numerical analysis and parameterization of the experimental neutron emission patterns measured in this work, a schematical procedure was applied, assuming a small number of representative moving sources which evaporate neutrons isotropically in the respective emitter frames of reference. Each of these hypothetical sources $s$ is characterized by its mass, $m_{p} A_{s}$, and its velocity, $v_{s}=\sqrt{2 \varepsilon_{s} / m_{p}}$, where $m_{p}$ is the mass of a nucleon and $\varepsilon_{s}=E_{s} / A_{s}$ stands for the kinetic energy per nucleon of each source. The Maxwell-Boltzmann-type energy spectrum of neutrons evaporated from each source is parameterized in terms of a temperature parameter $\mathrm{T}_{\mathrm{S}}$. It is advantageous to analyze the 
experimental neutron emission patterns in terms of Galilei-invariant neutron velocity $\left(\vec{v}_{n}\right)$ distributions of the form

$$
\frac{d m_{s}}{d \vec{v}_{n}}=\frac{d^{2} m_{s}}{v_{n}^{2} d \Omega_{n} d v_{n}}=\frac{m_{s}}{v_{n}} \frac{d^{2} m_{s}}{d \Omega_{n} d \varepsilon_{n}}
$$

In Eq.II.3, the double-differential neutron multiplicity can be written as

$$
\frac{d^{2} m_{s}\left(\theta_{n}\right)}{d \Omega_{n} d \varepsilon_{n}}=\frac{\bar{m}_{s}}{2\left(\pi T_{s}\right)^{3 / 2}} \sqrt{\varepsilon_{n}} \exp \left\{-\frac{E_{n}}{T_{s}}\right\}
$$

where $\bar{m}_{s}$ indicates the average multiplicity of neutrons emitted from source $s$, and the quantity $E_{n}$ denotes the neutron energy in the frame in which the source velocity is measured, i.e.,

$$
E_{n}=\varepsilon_{n}+\varepsilon_{s}-2 \sqrt{\varepsilon_{n} \varepsilon_{s}} \cdot \cos \theta_{n s}
$$

and the angle $\theta_{n s}$ is that between the velocity directions of the source and the neutron. The contour lines of the invariant differential multiplicity of neutrons evaporated from a single source are concentric circles, centered about the tip of the velocity vector of that source, in any inertial system. This existence of such a characteristic pattern makes it relatively simple to recognize the presence of one or even several emitters contributing to a velocity distribution, and to determine the parameters describing these sources. In the present work, superpositions of the contributions of up to three different sources to a neutron velocity distribution were considered. From fits of the experimental velocity distributions assuming two theoretical sources, representing neutron evaporation in flight from fully accelerated PLF and TLF produced in a binary collision, values for the emission angle $\left(\theta_{\mathrm{PLF}}\right)$ of the PLF and for the total kinetic-energy loss $E_{\text {loss }}$ were also obtained. For events, in which no massive PLF was identified, e.g., for triggers based on the detection of LCP's or IMF', the emission angle of the hypothetical emitting source was taken as $\theta_{\mathrm{s}} \approx$ $0^{\circ}$. In these fits, the finite experimental time resolution was taken into account by folding the corresponding theoretical velocity spectra with a Gaussian detector response function. 


\section{Calculations}

As indicated in the introduction, at comparatively low bombarding energies, i.e., a few $\mathrm{MeV}$ per nucleon above the barrier, many of the phenomena observed in heavy-ion interactions can be reproduced quantitatively in terms of the nucleon exchange model NEM [Ran82,87, Døs85]. The fundamental assumptions of this dominantly one-body, meanfield model are believed to be well justified at such low energies but can, a priori, not be expected to remain valid in the domain of Fermi bombarding energies, where two-body effects should become dominant. However, it is not precisely known, at what bombarding or excitation energies the NEM loses its validity. Furthermore, no quantitative dynamical reaction theory has been developed specifically for the Fermi energy regime as yet. For these reasons, it appears interesting to follow the performance of the NEM into the intermediate-energy regime and to test its predictive power for the present ${ }^{197} \mathrm{Au}+{ }^{208} \mathrm{~Pb}$ reaction. In the calculations referred to below, the model is used in a numerical implementation described in detail elsewhere [Sch87]. Here, only the main concepts of the NEM will be recalled.

The basic microscopic mechanism assumed in the NEM is the exchange of independent nucleons between the interaction partners. The properties of these fragments such as mass, charge, excitation energy and spin, as well as the overall shape of the intermediate dinuclear system, are changed as a result of a diffusion-like, stochastic nucleon exchange process. This process leads to the appearance of classical friction forces acting on the collective motion and a host of induced transport phenomena. As a consequence, the reaction variables, represented in the following by the set of macroscopic

observables $\bar{x}=\left\{x_{i}\right\}$, are not sharply defined in a collision but follow a time-dependent probability distribution $P(\bar{x}, t)$. In the NEM, the time dependence of this probability is taken to be determined by a Fokker-Planck equation,

$$
\frac{\partial}{\partial t} P(\vec{x}, t)=-\sum_{i} \frac{\partial}{\partial x_{i}}\left\{v_{i} P(\vec{x}, t)\right\}+\sum_{i, j} \frac{\partial^{2}}{\partial x_{i} \partial x_{j}}\left\{D_{i j} P(\bar{x}, t)\right\} .
$$

Here, the quantities $v_{i}$ and $D_{i j}$ denote the drift and diffusion coefficients, respectively, for the macroscopic observables $x_{i}$ and $x_{j}$. Form factors for these transport coefficients, containing information on the actual physics of the multi-nucleon exchange processes, have been derived in the microscopic theory of Randrup et al. [Ran82,87, Døs85]. The 
average values $\left\{\bar{x}_{i}, \dot{\bar{x}}_{i}\right\}$ of the coordinates follow a time dependence given by the LagrangeRayleigh equations of motion

$$
\left\{\frac{d}{d t} \frac{\partial}{\partial \dot{x}_{i}}-\frac{\partial}{\partial \bar{x}_{i}}\right\} \mathbf{L}=-\frac{\partial}{\partial \dot{x}_{i}} \mathbf{F}
$$

In the Lagrangian $\mathbf{L}=\mathbf{T}-\mathbf{V}$, the kinetic energy is denoted by $\mathbf{T}$, whereas $\mathbf{V}$ stands for the potential energy. In the adiabatic limit of the model, the interaction potential governing the relative motion is calculated from the liquid-drop model surface energy with proximity corrections and the Coulomb potential. A geometry is assumed for the dinuclear system in which spherical projectile-like and target-like fragments are connected by a cylindrical neck. This picture is in contrast with the non-adiabatic, sudden limit, in which the proximity potential and all other form factors of the NEM are calculated for overlapping, frozen-density configurations of the interacting nuclei. This non-adiabatic proximity potential has a hard core and is generally more repulsive than its adiabatic variant.

The Rayleigh dissipation function $\mathbf{F}$ appearing in Fig. III.2 describes frictional forces equivalent to "wall and window formula" [Blo78]. The classical formulation represented by Eqs. III. I and III. 2 is consistent with linear-response theory [Hof76,77]. In extensive studies carried out at lower bombarding energies, it was shown [Sch84] that several macroscopic degrees of freedom, such as the mass and charge asymmetries of the dinuclear system, develop in an overdamped motion determined by the dynamical driving forces

$$
F_{i}=\frac{\partial}{\partial \bar{x}_{i}} \mathbf{L} \cdot \frac{\partial}{\partial \dot{\bar{x}}_{i}} \mathbf{F}
$$

For small velocities along the coordinate $\bar{x}_{i}$, this force reduces to the familiar form $F_{i}=-\partial \mathrm{V} / \partial \bar{x}_{i}$, i.e., to the negative gradient of the corresponding driving potential. A more detailed discussion of the formalism and its numerical implementation can be found elsewhere [Sch84,87]. 
Since the system ${ }^{197} \mathrm{Au}+{ }^{208} \mathrm{~Pb}$, studied in the present work, is nearly symmetric in the entrance channel, the interacting system is not expected to develop significant average charge and mass asymmetries, which are represented in the following by the average atomic and neutron numbers, $\bar{Z}_{P L F}$ and $\bar{N}_{P L F}$, respectively. This expectation is supported by the potential energy surface (PES) $\mathrm{V}\left(\bar{N}_{P L F}, \bar{Z}_{P L F}\right)$, calculated from the liquid-drop model with shell corrections, for an angular momentum of $\ell=0$ and a separation distance of $r=R_{\text {Sa. }}$. This PES is plotted in Fig.III.I in form of a contour diagram vs. neutron and atomic number of the PLF. The numbers at the contours are given in units of MeV. As can be seen from this figure, the entrance-channel projectile-target combination ("injection point") is located in close proximity of the minimum ("symmetry point") of a rather shallow PES. There is essentially no gradient in the PES at the injection point which could be expected to cause a substantial average "drift" in the distributions in atomic or neutron numbers. In particular, there is no trend in the PES favoring the formation of a fused, mononuclear system. In addition, because of the strong Coulomb and centrifugal forces, the interaction potential becomes increasingly repulsive at smaller separation distances or larger $\ell$-values, inhibiting strongly, and for all impact parameters, the formation of an equilibrated, fused composite nucleus. In the model, such a mononuclear system can only be produced with very small cross sections, corresponding to the extreme wings of the fragment probability distribution.

The model calculations predict a scenario that is well-known from lower bombarding energies [Sch84]. Fragment $\mathrm{A}$ and $\mathrm{Z}$ distributions are bimodal for all impact parameters, with most probable values centered at those of projectile and target. With decreasing impact parameter, experimentally identifiable by increasing degrees of energy damping or increasing multiplicities of the associated light particles, the $\mathbf{Z}$ or $\mathrm{A}$ distributions of the PLF's or those of the TLF's are predicted to broaden substantially. This feature is indicative of the time evolution of diffusion-like multi-nucleon exchange processes, with similar probabilities for nucleon stripping and pickup. distributions predicted by this model for the ${ }^{197} \mathrm{Au}+{ }^{208} \mathrm{~Pb}$ reaction will be compared to experimental data in Section IV.

In order to provide an orientation with respect to the time scales, on which various processes associated with ${ }^{197} \mathrm{Au}+{ }^{208} \mathrm{~Pb}$ collisions at $\mathrm{E} / \mathrm{A}=29 \mathrm{MeV}$ are predicted to evolve, results of dynamical NEM calculations for a representative dissipative, $\ell=1.45$ $\mathrm{k} \hbar$, collision are displayed in Fig.III. 2 vs. time. The time evolution of the dissipated 
energy $E_{\text {diss }}\left(=E_{\text {loss }}\right.$ ) is depicted in the top panel of this figure. As can be seen, $E_{\text {diss }}$ is essentially zero for $t<6 \cdot 10^{-22} \mathrm{~s}$, defining the beginning of the dissipative interaction. With increasing interaction time, the dissipated energy increases drastically, even beyond the classical turning point of the trajectory which is indicated by a vertical arrow. At breakup of the system at $t \approx 1 \cdot 10^{-21} \mathrm{~s}$, corresponding to an interaction time of $t_{\mathrm{int}} \approx 4 \cdot 10^{-22} \mathrm{~s}$, the collision has eventually dissipated an energy of $E_{\text {diss }} \approx 970 \mathrm{MeV}$. As will be discussed further below, such an energy loss is sufficient for the evaporation of more than 60 neutrons from the reaction system, along with a smaller, but significant number of charged particles. Since the beam velocity is relatively high, such a significant energy loss corresponds to only a small change in the relative velocity $v_{\text {rel }}$. This effect is observed in the second panel from the top of Fig.III.2, where a change from the beam velocity of $v_{\text {rel }}$ $=7.2 \mathrm{~cm} / \mathrm{ns}$ to the asymptotic velocity of the accelerated fragments of $v_{\mathrm{rel}} \approx 6 \mathrm{~cm} / \mathrm{ns}$ is predicted for this collision. One also notices a relatively small change in $v_{r e l}$, after the breakup of the dinuclear system, which is due to acceleration of the final fragments in their mutual Coulomb field.

As seen from the third panel of Fig.III.2, the nuclear temperature increases with the dissipated energy, reaching eventually a moderate value of $\tau \approx 4 \mathrm{MeV}$, for this collision, as calculated from the relation $\tau=\sqrt{E_{\text {diss }} / a}$ involving the level density parameter $a \approx \mathrm{A} / 8$ $\mathrm{MeV}^{-1}$ of the dinuclear system. In the model calculation, the somewhat idealized assumption is made that the intrinsic excitation energy of each fragment equilibrates instantaneously. A small number of preequilibrium particles [Bla75,81,85] is actually predicted by the NEM [Ran90] to be emitted on a time scale faster than that of the thermal equilibration process. This "Fermi jet" emission process will be discussed further below.

Because of the symmetry of the system, the individual temperatures acquired by PLF and TLF in the course of a collision are predicted to be very similar to each other. The excitation energies can be rather high, leading a substantial evaporative mass loss of the fragments or to fisssion. These decay processes are expected to occur largely after the reaction has subsided. Since the temperatures reached even in highly dissipative ${ }^{197} \mathrm{Au}+{ }^{208} \mathrm{~Pb}$ collisions are only moderate, the particle evaporation times are expected to be long compared to the interaction times. This fact is illustrated, for a highly dissipative collision, in the bottom panel of Fig.III.2, where the n utron evaporation time $t_{n}$ is plotted vs. time elapsed in the collision. This evaporation time is calculated from the neutron decay 
width $\Gamma_{\mathrm{n}}$, according to $t_{n}=\hbar / \Gamma_{\mathrm{n}}$, as function of the excitation energy $E$, where $\Gamma_{\mathrm{n}}$ is given by

$$
\Gamma_{n}=\frac{A^{2 / 3}\left(E-B_{n}\right)}{\pi a_{n} \kappa_{0}} \cdot \frac{\exp \left[2 \sqrt{a_{n}\left(E-B_{n}\right)}\right]}{\exp [2 \sqrt{a E}]}
$$

In eq.III.4, the quantity $B_{n}$ is the neutron binding energy, the quantities $a$ and $a_{n}$ denote the level density parameters for the emitter fragment and its daughter, and $\kappa_{0} \approx 10 \mathrm{MeV}$ is a constant. As can be inferred from Eq.III.4, the evaporation time is a highly non-linear function of the excitation energy, increasing approximately exponentially with the number of neutrons evaporated. Therefore, the following arguments apply to the lower limits estimated for the average neutron evaporation times.

As can be seen from Fig.III.2, throughout most of the collision considered, the mean neutron evaporation time is several orders of magnitude longer than the total interaction time, such that one expects less than one neutron to be emitted during this time, i.e., from the intermediate system. Similar considerations apply to neutron emission during the acceleration time, since the latter time is of the same order as the interaction time, as results displayed in the figure clearly show. At most a few (of the more than 60 ) neutrons could be evaporated from the reaction fragments before full Coulomb acceleration has occurred. However, it would experimentally be very difficult to distinguish such neutrons from those emitted from the asymptotic fragments, since the change in relative fragment velocity during the acceleration phase is relatively small.

The general situation described above is not expected to change qualitatively for smaller impact parameters, since, as will be demonstrated in Section IV below, the associated energy losses are measured to be very similar to that associated with the representative collision illustrated in Fig. III.2. The model predictions apply similarly to charged-particle emission. Since the evaporation of charged particles is suppressed by relatively high Coulomb barriers, their mean emission times are expected to be even longer than those of the neutrons. This observation should apply in particular to the heavier charged particles, the intermediate-mass fragments (IMF). Consequently, one then expects a negligible 
probability for the "prompt" disassembly or multi-fragmentation of the intermediate dinuclear system via statistical, evaporation-like processes.

The above model calculations then predict, for all impact parameters, a basically binary process producing hot primary PLF and TLF which subsequently decay. This statement is rignrously true only as far as the number of massive fragments is concerned. As already stated earlier, the NEM predicts [Bon80, Ran87] a relatively small number of preequilibrium particles to be emitted essentially promptly as "Fermi jets", i.e., during the interaction time. A direct emission of nucleons in such jet processes is physically possible, because of the boost imparted on a transferred nucleon by the relative motion. The emission rate of such Fermi-jet particles is, hence, governed by the nucleon exchange currents. However, not all of the nucleons transferred to continuum states will actually be emitted directly in a Fermi jet. The nucleon-nucleon scattering processes occurring in the recipient nucleus, reflected in a finite mean free path of a transferred nucleon, attenuate the flux of particles traversing the nucleus. Finally, refraction and reflection at the edge of the nuclear potential further reduce the probability for direct nucleon emission. The Fermi jet emission rate and the energy spectrum of the emitted particles depend critically on the velocity of a transferred nucleon in the recipient nucleus, which is given by the vector sum of nucleonic Fermi velocity and the velocity of relative motion. Since the nucleonic velocity distribution develops a high-velocity tail with increasing temperature of the nucleus, the jet emission rate is expected to increase with increasing energy dissipation, until the relative velocity starts decreasing significantly. An emission of complex particles in Fermi jet processes is not considered in this one-body nucleon exchange model. In this scenario, preequilibrium emission of complex-particles would require a prior, unlikely coalescence of groups of nucleons with small relative momenta.

To provide an idea of the importance of Fermi jet emission in the reaction ${ }^{197} \mathrm{Au}+{ }^{208} \mathrm{~Pb}$ at $\mathrm{E} / \mathrm{A}=29 \mathrm{MeV}$ and of the corresponding time scales involved, results of a model calculation are depicted in Fig. III.3, for a highly dissipative, $\ell=1.4 \mathrm{k} \hbar{ }^{197} \mathrm{Au}+{ }^{208} \mathrm{~Pb}$ collision. The top panel of this figure illustrates the time evolution of the relative distance $\mathrm{R}$ of PLF and TLF, and the geometrical and of the effective neck radius, $\mathrm{C}$ and $\mathrm{C}_{\text {eff }}$, respectively. The middle panel shows the time dependence of the average nuclear temperature $\tau$, whereas the bottom part of Fig. III.3 depicts the time-dependent rate of the approximately 2 PLF neutrons emitted in a Fermi jet. Obviously, this latter rate is always relatively small but has a maximum of $v_{\text {proj }} \approx 0.2 \cdot 10^{22} \mathrm{~s}^{-1}$ at $t=1 \cdot 10^{-22} \mathrm{~s}$, i.e., during the approach phase. For comparison, the classical turning point of the trajectory 
for relative motion is reached in this collision at $t \approx 1.8 \cdot 10^{-22} \mathrm{~s}$. Nevertheless, a considerable amount of energy has already been dissipated prior to Fermi jet emission, as is qualitatively expected. Consequently, if one were to define a single, effective source for these jet particles, as often done in interpretations of experimental data, this source would have to be associated with an average velocity reduced by $15 \%-20 \%$ (cf. Fig. III.2, second panel) from that of the beam. In the rest frame of this hypothetical source, the energy of the jet particles can be relatively high, of the order of several times the kinetic energy per nucleon of the effective source. In the laboratory system, however, the energies of the jet particles can be exceeded by those of particles evaporated at forward angles from fastmoving PLFs. Examples of the energy spectra of jet neutrons predicted for the present reaction at various angles will also be given and discussed in the following Section IV.

\section{Experimental Results and Discussion}

\section{IV.1. Energy and Atomic-Number Distributions of Charged Products}

Most of the charged-product data discussed in this work were taken with the PLF1 telescope at $\theta=-6.5^{\circ}$ and the Si-strip detectors placed on the other side of the beam, at "positive" angles. This telescope had an angular acceptance of $\Delta \theta= \pm 0.6^{\circ}$ and covered the quarter-point (or grazing) angle $\left(\left|\theta_{1 / 4}\right|=6.3^{\circ}\right)$ for this reaction (cf. Table 1). About this angle, one expects to sample a large range of kinetic-energy losses and, hence, a particularly broad spectrum of reaction products. This expectation is met by the experimental data for the PLFl telescope shown in Fig. IV.1, where the fragment yield is plotted as a two-dimensional contour diagram $v s$. atomic number and laboratory kinetic energy. One observes fragments in the entire range of $Z$-values, from IMF's with $Z=6$, the lower cutoff of this telescope, to the atomic number of the projectile $(Z=82)$, and kinetic energies ranging from very low values to those of elastically scattered projectiles. There are two instrumental artifacts visible in the distribution of Fig. IV.1. The horizontal ridge at $Z \approx 82$ is due to slit scattering, a diagonal gap visible in the distribution for fragment $Z$-values between 8 and 40 is caused by a deadlayer in one of the elements of this telescope. Neither of these deficiencies is of consequence for the conclusions drawn in this paper. 
The data obtained with the telescope placed at $\theta \approx+8.5^{\circ}$ appear qualitatively similar to those obtained with the above telescope, except that the cross section for quasi-elastic events is much smaller in this detector, as expected from the detector location slightly backward of the grazing angle. This trend is seen continued in the data obtained with the telescope at the more backward angle of $\theta \approx-21.5^{\circ}$, where the fragment distribution no longer contains massive fragments that are clearly identified as PLF's. Here, the distribution is dominated by intermediate-mass fragments (IMF), with a cross section decreasing at first $(6 \leq Z \leq 30)$ approximately exponentially with atomic number, followed by a faster decrease for atomic numbers of $Z \geq 40$. The data for both these telescopes exhibit trends very similar to those obtained with the most forward telescope. However, since the latter set is more complete and extensive, the following discussion will concentrate on the $\theta \approx-6.5^{\circ}$ data.

The most prominent structure in the $\theta \approx-6.5^{\circ}$ data displayed in Fig. IV.1 is the peak of quasi-elastic events located at $Z \approx 82$ and $E \approx 6 \mathrm{GeV}$, from which a ridge of yield extends to lower $Z$ values and lower energies. Superimposed on this ridge, in the region of atomic numbers of $Z \approx 40$, one observes a fragment group with kinetic energies of the order of $E$ $\approx 1.5-2 \mathrm{GeV}$, and another one at $\mathrm{E} \approx 3.5-4 \mathrm{GeV}$. These two fragment components are attributed to sequential fission of the fast-moving PLF, corresponding to forward and backward emission, respectively. This was verified in the experiment, where it was possible to measure the two fission fragments from the decay of an excited PLF in coincidence with the two telescopes at $\theta \approx-6.5^{\circ}$ and $\theta \approx+8.5^{\circ}$. The properties of these fission components will not be further evaluated in this article, they are discussed in some detail elsewhere [Bre92, Que92]. However, it is of interest in the context of the present discussion to note that the cross section for sequential PLF fission is large, connecting this process mainly with quasi-elastic or partially damped primary collisions. Consequently, the domain of PLF's associated with this class of primary reaction events must be significantly depleted by sequential fission. More direct evidence for this conclusion is provided by the low multiplicity of accompanying neutrons, as will be discussed further below.

Apart from the slight complication due to the PLF fission fragments, the data shown in Fig. IV.1 represent a remarkably well defined correlation between atomic number $\mathrm{Z}$ and kinetic energy $E$ of the fragments. This correlation prescribes an almost straight-line diagonal in this plot, i.e., $E / Z \propto v^{2} \approx$ const. Such a correlation is indicative of reaction products with velocities $v$ similar to that of the projectile, if the mass of a product is 
roughly proportional to its atomic number. As illustrated in the insert of Fig. IV.1, the diagonal ridge of yield seen in this figure is actually understood to be the remainder of the PLF distribution that did not undergo sequential fission and was only affected by particle evaporation processes. As described quantitatively by the NEM, discussed in Section III, the reaction leads to a distribution of primary PLF's and TLF's that are always centered about the masses and atomic numbers of projectile and target, respectively. However, with increasing interaction time, signaled, e.g., by increasing amounts of dissipated kinetic energy of relative motion, these distributions are predicted to broaden significantly. One of these ZPL distributions are represented by the shaded Gaussian ("all PLF") in the insert of Fig. IV.1. The hot primary fragments decay sequentially in flight by particle evaporation or fission. The competition between these two decay modes depends [Van73] sensitively on the fissility, or the $Z^{2} / A$ value, of the excited fragment. For the relatively heavy, high-Z PLF's which have also high fissilities, fission is the dominant decay channel. Consequently, these heavy fragments undergo fission, leaving only the significantly lighter PLF's. The distribution of these latter fragments is illustrated by the solid black Gaussian in Fig. IV.1, which appears shifted on average by $\Delta Z$ towards atomic numbers smaller than the centroid of the primary distribution.

The above reaction scenario was modeled quantitatively and, in Fig. IV.1, results of corresponding calculations, represented by a solid curve, are compared to the experimental E-Z correlation. The NEM in its non-adiabatic limit (cf. Section III) was used to calculate [Sch87] the primary PLF distribution in mass and atomic number, APLF, $Z_{\mathrm{PLF}}$, respectively, as well as the fragment excitation energies, . The predicted variation of this latter quantity along the ridge line is illustrated by the arrows in Fig. IV.1. The sequential statistical decay of the reaction primaries was modeled with the code EVAP [Nic92]. These calculations are realistic and were carried out only for relatively low fragment excitation energies $\left(E^{*} \leq 200 \mathrm{MeV}\right)$. At higher excitation energies and associated spins, the interplay between statistical decay and dynamical effects [Hil93], as well as the competition between particle decay and fission, are not well understood and not modeled adequately in the evaporation calculations predicting fission decay for all fragments. But for the above limited range of relatively low excitation energies and associated spins, the average $\mathrm{Z}_{\mathrm{PLF}}-\mathrm{E}_{\mathrm{PLF}}$ correlation, given by the position of the ridge in Fig. IV.1, is rather well described by the above calculations. In addition, the width of the cross section ridge is well understood, in these calculations. However, for lower $\mathrm{Z}$ or $\mathrm{E}$ values, significant quantitative deviations of the calculations from the data do occur, which could mostly be due to insufficiencies in the application of the statistical model.. 
Calculations with the NEM in its adiabatic limit (cf. Section III) lead to results that are qualitatively similar to those discussed above. However, they do not reproduce the experimental ZPLF-EPLF correlation of Fig. IV.I quantitatively. Since, for a given total kinetic energy loss, this latter type of model calculation predicts significantly less nucleon exchange than the non-adiabatic model, it underestimates the widths of the fragment $\mathrm{Z}$ distributions and, hence, predicts a weaker correlation than observed experimentally.

The process of a binary damped collision, followed by sequential fission or particle evaporation, discussed above provides a consistent explanation for the appearance of the experimental correlation between fragment $\mathrm{Z}$ and $\mathrm{E}$ shown in Fig. IV.1. Although this observation can not yet he taken as a positive identification of the origin of the experimental Z-E correlation, other model explanations that have previously been invoked in the literature for similar experimental results obtained for other systems can be rejected, for the present ${ }^{197} \mathrm{Au}+{ }^{208} \mathrm{~Pb}$ reaction. For example, the strong Z-E correlation for PLF's represented by the narrow ridge in Fig. IV.1 cannot be explained in terms of a drift with increasing energy loss of the primary Z $\mathrm{PLF}$ distribution. Due to the mass symmetry of the system, no effective driving force for proton transfer is expected. This expectation is verified by the predicted potential energy surface (PES) for nucleon transfer depicted in Fig. III.1. One observes from this figure that the injection point is located very closely to the shallow minimum of the PES. Consequently, there is no energetically preferred direction for the transfer of protons. For the same symmetry argument, there is no obvious reason for asymmetric projectile fragmentation or massive-transfer processes that could lead to an Z-E correlation of the type observed.

The discussion of data and calculations, presented in this section for the reaction ${ }^{197} \mathrm{Au}+{ }^{208} \mathrm{~Pb}$ at $\mathrm{E} / \mathrm{A}=29 \mathrm{MeV}$, has provided ample supporting evidence for the prevalence of a binary collision dynamics, for a heavy, symmetric reaction system. For rather peripheral, partially damped events $\left(E_{\text {loss }} \leq 350 \mathrm{MeV}\right)$, the binary reaction scenario has been demonstrated directly by a kinematical-coincidence measurement. These measurements also provide good estimates of the excitation energies of the primary fragments, which are shown to acquire excitation energies of the order of $\approx \approx E_{\text {loss }} / 2$. Such collisions are quantitatively very well described by a damped or dissipative reaction mechanism, as modeled by the NEM, which is rather successful also in the reproduction of low-energy data. The experimental Z-E correlation measured at forward angle appears to be essentially a result of the depletion of the PLF distribution by fission. This correlation 
provides evidence for a finite width of the primary PLF-Z distribution, already according to very general symmetry arguments. As predicted by the NEM, this width increases with decreasing PLF energy or increasing energy loss. This feature is actually borne out by the present experiment which suggests a new and effective method to measure the widths of primary fragment $\mathrm{A}$ and $\mathrm{Z}$ distributions. For more dissipative collisions, in which the massive fragments detected in the forward-angle telescope are significantly lighter than the projectile, this low-energy reaction model is quantitatively less successful in its predictions.

\section{IV.2. Exclusive Neutron Multiplicity Distributions}

The discussion presented in the previous subsection has demonstrated the binary, dissipative character of relatively peripheral collisions leading to massive fragments $(\mathrm{Z} \geq$ $70)$ at forward angles, as was directly proven by kinematical-coincidence measurements. Because of the sequential disintegration of the hot reaction primaries, such measurements are generally feasible for the ${ }^{197} \mathrm{Au}+{ }^{208} \mathrm{~Pb}$ reaction only up to moderate total kineticenergy losses of $E_{\text {loss }} \leq 350 \mathrm{MeV}$, while for the special class of PLF-fission events, this statement holds for energy losses $E_{\text {loss }} \leq 570 \mathrm{MeV}$. The measurement of associated neutrons enables one to obtain a more general survey over the development of the dissipative features over the whole range, and for all events, as will be shown in the following. The multiplicity of neutrons has been demonstrated [Gue89, Gal89, Pia90, Sch92] to be a sensitive observable indicating the degree of energy dissipation, more generally applicable than observables based on charged-particle multiplicities.

An experimental survey of the associated neutron multiplicities for fragments detected at $\theta \approx-6.5^{\circ}$ is given in Figs. IV.2 to IV.4. In Fig. IV.2, the two-dimensional experimental correlation between atomic number and laboratory energy is only coarsely outlined (CF. Fig. IV.1), superimposed by a grid showing the multiplicity $m_{\text {tot }}$ of coincident neutrons, averaged over the corresponding Z-E bin. The neutron multiplicity was obtained from an integration over angle and neutron spectrum, a procedure explained in detail in Section II.2 (cf. Eq. II.2). Several trends are obvious in the neutron multiplicity function $\mathrm{m}_{\mathrm{tot}}(\mathrm{Z}, \mathrm{E})$. Starting from the region of quasi-elastic events, the quantity $\mathrm{m}_{\mathrm{tot}}$ increases at first monotonically along the intensity ridge, with decreasing $Z$ and $E$, reaching a maximum of $\left(\mathrm{m}_{\mathrm{tot}}\right)_{\max } \approx 67$ at $\mathrm{Z} \approx 60$ and $\mathrm{E} \approx 2.5 \mathrm{GeV}$. This hehavior of the neutron multiplicity is as expected from the increasing dissipation associated with decreasing $Z$ and $\mathrm{E}$ of the fragments. After that, there is an approximately $15 \%$ decrease in $\mathrm{m}_{\mathrm{tot}}$ from its maximum value. Apparently, fragments with $Z$ values in the range $30 \leq Z \leq 40$ are 
characterized by similar degrees of energy dissipation as the more massive fragments emerging from partially damped events. This behavior is understood as being due to the presence of fragments from fission of quasi-elastic and partially damped PLF's, in this Z-E region. The dominant origin of the fission fragments from the decay of PLF's produced in peripheral collisions is demonstrated by the low neutron multiplicities observed for the faster of the two fission fragment components, which is less contaminated by non-fission events than the slower component.

After passing this fission region, the multiplicity $m_{\text {tot }}$ increases again with decreasing $\mathrm{Z}$ and $E$, up to its previous maximum value. It is interesting to note that the average fragment $Z$ values of $Z \approx 15-20$, in this domain of fast, relatively heavy, intermediatemass fragments (IMF), and those of the more massive PLF's $(Z \approx 60)$ with similarly high degrees of dissipation add approximately up to the atomic numbers of the projectile. This observation suggests that hoth types of charged products emerge from the same type of events. It confirms the resuis of a fragment-fragment coincidence measurement discussed in the previous section, which indicated the presence of asymmetric, fission-like decays of partially damped PLFs. For the smallest $Z$ values measured with the forward telescope PLF1, there is again a slight drop in $\mathrm{m}_{\text {tot }}$. This observation is not unexpected, since the lightest charged particles are emitted most frequently, and from a variety of primary and secondary products. Their cross section is, hence, dominated by the large intensities of partially damped events, which are associated with relatively low neutron multiplicities. As can also be expected, for a fixed $\mathrm{Z}$ value, Fig. IV.2 demonstrates that the associated neutron multiplicity increases with decreasing fragment energy, i.e., with increasing degree of dissipation.

Projections of the two-dimensional distribution $\mathrm{m}_{\mathrm{tot}}(\mathrm{Z}, \mathrm{E})$ of the multiplicity of associated neutrons, illustrated in Fig. IV.2, onto the $\mathrm{Z}$ and $\mathrm{E}$ axes are depicted in Figs. IV.3 and IV.4, respectively. In Fig. IV.3, the intensity-weighted average associated-neutron multiplicity,

$\left\langle\mathrm{m}_{\mathrm{n}}\right\rangle$, is plotted $v$ s. the average fragment atomic number, $\langle\mathrm{Z}\rangle$. The experimental data are represented by the histogram in Fig. IV.3, while calculations carried out with the NEM are shown as solid dots. The data illustrate a bimodal multiplicity distribution, which indicates the presence of two fragment groups of similar multiplicities or degree of damping. As is argued above and in Section IV.1, the light and heavy fragment components can be understood as resulting from the asymmetric, fission-like decay of excited PLF's. One then concludes that, for the present ${ }^{197} \mathrm{Au}+{ }^{208} \mathrm{~Pb}$ reaction, the 
atomic number of a reaction product is not a good indicator of dissipated energy or other quantities correlated with it, such as the collision impact parameter.

The correlation between average neutron multiplicity, $\left\langle\mathrm{m}_{\mathrm{n}}\right\rangle$, and average measured laboratory kinetic energy, $\langle E\rangle$, of the fragments detected at $\theta \approx-6.5^{\circ}$ is depicted in Fig. IV.4. as a histogram, while the dots represent again NEM simulation calculations. In this figure, one observes a drastic increase in the average multiplicity, with the fragment kinetic energy decreasing from 6 to $4 \mathrm{GeV}$. There appears to be a definite change in slope of the correlation, with a relatively small increment $\left|\Delta<m_{n}>\right| \Delta<E>\mid$ at the low kinetic energies, or high degrees of dissipation. This effect is understood to be mostly due to the competition by charged-particle emission, which becomes important at the higher energy losses [Sch92]. Partially, this effect is caused by the recoil imparted on the evaporating fragment by the emitted particles, reducing the measured fragment laboratory kinetic energy by a fraction proportional to the evaporated mass. Hence, in Fig. IV.3, the dissipated energy, or energy loss, increase from right to left in a non-linear fashion.

Simulation calculations were performed, based on the NEM and modeling the decay of the primary fragments with an evaporation code, as discussed in Section III. The results are shown in Figs. IV. 3 and IV.4 as solid dots. As explained earlier, these simulation calculations can only be carried out for a limited range of fragment excitation energies, because of insufficiencies in the treatment of their sequential decay. For the domain of partially damped events, where these calculations are applicable, one observes good agreement between data and model predictions. This is true, both for the correlation $<\mathrm{m}_{\mathrm{n}}$ $>(\langle\mathrm{Z}\rangle)$ and the dependence of $\left\langle\mathrm{m}_{\mathrm{n}}>\mathrm{on}\right.$ average kinetic energy $\langle\mathrm{E}\rangle$, lending further support for the validity of a dissipative reaction mechanism operating at least in peripheral ${ }^{197} \mathrm{Au}+{ }^{208} \mathrm{~Pb}$ collisions.

Data equivalent to those measured in coincidence with fragments detected at $\theta \approx-6.5^{\circ}$ are found in coincidence with trigger fragments measured with the telescopes PLF2 and PLF3, located at angles of $\mathrm{e}=+8.5^{\circ}$ and $\theta \approx-21.5^{\circ}$, respectively. The associatedneutron multiplicities are similar for similar fragment types. It is noteworthy, however, that fragments measured at the largest angle of $\theta \approx-21.5^{\circ}$ are associated on average with somewhat higher neutron multiplicities than those detected at the more forward angles. For example, an associated-neutron multiplicity of $\mathrm{m}_{\mathrm{tot}} \approx 74$ is measured at $\theta \approx-21.5^{\circ}$, for fragments with $\mathrm{Z} \approx 30$ and $\mathrm{E} \approx 1.2 \mathrm{GeV}$. At this angle, the correlation $\left.<\mathrm{m}_{\mathrm{n}}>(<\mathrm{Z}\rangle\right)$ 
and the dependence of $\left\langle\mathrm{m}_{\mathrm{n}}>0\right.$ n average kinetic energy $<\mathrm{E}>$ are both relatively weak. At present, these interesting observations are not fully understood.

In summary of this subsection, examples have been presented of how knowledge of the multiplicity of neutrons associated with charged reaction products can be exploited to explore the origin of these fragments in peripheral or more dissipative collisions. For example, the bimodal multiplicity distribution measured in coincidence with fragments at $\theta \approx-6.5^{\circ}$, indicates that two fragment groups of very different mass and kinetic energy, are produced in events with similar degree of damping, i.e., in collisions with similar impact parameters. Very likely, these fragments are produced in the same reaction events, e.g., via sequential decay processes. Hence, for the present ${ }^{197} \mathrm{Au}+{ }^{208} \mathrm{~Pb}$ reaction, the atomic number of a reaction product is not a good indicator of dissipated energy or collision impact parameter. There is, however, a definite correlation observed between neutron multiplicity and the final fragment laboratory energy, an observable that may be of practical value for selecting certain classes of reaction events. The experimental correlations between average associated-neutron multiplicity, $\left\langle\mathrm{m}_{\mathbf{n}}\right\rangle$, average measured laboratory kinetic energy, $\langle E\rangle$, and atomic numbers, $\langle Z\rangle$, of the fragments measured for the reaction ${ }^{197} \mathrm{Au}+{ }^{208} \mathrm{~Pb}$ at $\theta \approx-6.5^{\circ}$ or $+8.5^{\circ}$ are found to be well represented by simulation calculations based on the NEM, as long as fragment properties are accessible by evaporation calculations.

\section{IV.3. Exclusive Neutron Velocity Distributions}

As exemplified in the preceding section, the integrated number of neutrons emitted in a reaction event provides an important analytical tool for the exploration of the underlying reaction dynamics, based on the close correspondence between neutron multiplicity and excitation energy of the reaction products, total dissipated energy, and collision impact parameter. The present experiment did not have enough detection efficiency to provide a model-independent measure of the total neutron multiplicity, for all types of reaction events, but was designed to determine the neutron angle-velocity emission patterns in considerable detail. Much more so than the total multiplicities do experimental neutron emission patterns allow one to draw definite conclusions with respect to the temperatures and kinematical properties of one or several neutron emitters present in a reaction. Of course, in an energetic reaction between two very heavy nuclei, there are potentially a multitude of particle emitters present in the exit channel. Nevertheless, as explained in 
Section II.2, the use of the somewhat artificial construct of an "effective" moving source, representing actually a whole distribution of such emitters, can be regarded as justified, for the practical purposes of data analysis. Experimentally, the tasks of identifying different particle emitters produced in a collision and to evaluate its properties are significantly facilitated, if the emission patterns of the coincident particles are presented in a Galilei-invariant form (cf. Eqs. II.3 and II.4). This is illustrated in the following by the qualitative discussion of a sequence of neutron emission patterns, for events with increasing degree of dissipation. Further below, numerical fit results will be presented, in a more quantitative discussion.

In Fig. IV.5, linear, two-dimensional maps are shown of the Galilei-invariant cross section $d^{2} \sigma / v_{n x} d v_{n x} v_{n z}$ of neutrons in coincidence with fragments from the reaction ${ }^{197} \mathrm{Au}+{ }^{208} \mathrm{~Pb}$ at $\mathrm{E}_{\mathrm{lab}} / \mathrm{A}=29 \mathrm{MeV}$, measured at $\theta \approx-6.5^{\circ}$. These velocity distributions are plotted in form of contour diagrams $v s$. the neutron velocity components parallel $\left(v_{n x}\right)$ and perpendicular $\left(v_{n y}\right)$ to the heam, respectively. Here, the $x-y$ coordinate plane is defined by the PLF telescopes and most of the neutron detectors. Panels a) and b) of Fig. IV.5 are theoretical distributions, while the distributions e) - $f$ ) are experimental, as measured for various conditions on the "trigger" fragments detected with the PLF1 telescope. The arrows in Fig. IV.5a indicate the velocities of PLF, TLF, and of the c.m. system in the laboratory. This velocity distribution was generated in a simulation calculation assuming sequential neutron emission from fully accelerated projectile and target fragments, isotropic in the fragments' rest frames. The fragments are taken to emerge from a peripheral collision with an energy loss of only $E_{\text {loss }}=50 \mathrm{MeV}$. The resulting bimodal neutron emission pattern with two sets of concentric contour lines, centered at the tips of the PLF and TLF velocity vectors, respectively, is quite characteristic and provides an obvious identification of the velocity vectors of these two emitters. The high PLF velocity is seen to lead to a strongly forward peaked angular distributions of neutrons from this emitter, whereas neutrons from the slow TLF are evaporated almost isotropically in the laboratory. From the measured velocities associated with the centroids of the two invariant velocity components, one can determine the relative velocity and the energy loss suffered in a collision. Folding the theoretical distribution of Fig. IV.5 a with the response of the 21 neutron detectors results in the theoretical invariant distribution depicted in Fig. IV.5 b. Here, the finite granularity of the TOF spectrometer is seen to lead to a degeneration of the circular pattern of the neutrons from the PLF to a wedge-like shape at forward angles. The velocity distribution of neutrons from the TLF, on the other hand, is 
largely preserved in its symmetry but significantly affected by the finite detection thresholds.

The experimental emission pattern of neutrons observed in coincidence with PLF's of $77 \leq$ $\mathrm{Z} \leq 83$ is shown in Fig. IV.5 c. As expected from such a condition selecting mainly peripheral collisions, this emission pattern has two components and is in appearance very similar to the theoretical distribution of Fig. IV.5 b. An average total neutron multiplicity of $m_{\text {tot }}=(15 \pm 1)$ is obtained for these events. In addition, the similar intensities of the two individual components indicate that the corresponding emitters, the primary PLF and TLF, have comparable total excitation energies. This feature is again consistent with a dissipative reaction mechanism in peripheral collisions and contradicts the notion of projectile fragmentation processes.

The neutron emission pattern measured in coincidence with the high-velocity component of fission fragments centered at $\mathrm{E} \approx 3.5 \mathrm{GeV}$ (cf. Fig.IV.1) is shown in Fig. IV.5 d. These collisions are associated with an average multiplicity of $m_{t o t}=(45 \pm 4)$; they are, therefore, partially damped reactions, associated with somewhat higher degrees of dissipation than those pictured in Fig. IV.5 c. As observed from Fig. IV.5 d, the neutron distributions associated with PLF fission are relatively broad, as can generally be expected from the broader PLF distributions and higher excitation energies associated with higher degrees of dissipation. It is noteworthy, however, that the velocity distribution is still bimodal, although there are now at least three massive fragments in the exit channel. This observation is consistent with established systematics [Hil93], according to which neutrons accompanying fission at high excitation energies occurs overwhelmingly in a prescission mode. Accordingly, the distribution of Fig. IV.5 d was analyzed assuming that all neutrons are emitted from the PLF prior to its scission. The numerical analysis of the velocity distribution in terms of two moving sources (cf. Eqs.II.3-II.5) resulted in effective temperature parameters of $\mathrm{T}_{\mathrm{PLF}} \approx \mathrm{T}_{\mathrm{TLF}}=(3.1 \pm 0.5) \mathrm{MeV}$, as obtained from the slopes of the invariant distributions. The centroids of the velocity components are consistent with an energy loss of $E_{\text {loss }}=(600 \pm 200) \mathrm{MeV}$, a value consistent with the value of $E_{\text {loss }}$ determined [Bre92] from the kinematics of these PLF fission fragments. The errors quoted in the above values of multiplicity and effective temperature parameters are those resulting from the applied fit procedure and do not account for the overall uncertainty in the neutron detection efficiency discussed in Section II.2. 
In Fig. IV.5e, the invariant velocity distribution is shown for neutrons in coincidence with massive fragments in the range $58 \leq \mathrm{Z} \leq 64$ measured at $\theta \approx-6.5^{\circ}$. These types of massive fragments are associated with the highest total neutron multiplicities observed in this work. The corresponding velocity distribution has qualitatively a bimodal appearance similar to that associated with fission events. Again, the fast-neutron component is aligned with respect to the direction of the detected trigger fragment. However, its overali shape seems to be more compact, an effect of a smaller relative velocity of the two emitters signalizing a higher degree of dissipation than the latter type of events. Finally, Fig. IV.5 $\mathrm{f}$ presents the invariant velocity distribution of neutrons coincident with intermediate-mass fragments (IMF) with $5 \leq Z \leq 19$, detected at $\theta \approx-6.5^{\circ}$. These events, too, are associated with very high energy losses. From the velocity distribution of Fig. IV.5 f, it is obvious that even for highly dissipative events triggered by IMF's, a basic bimodal pattern persists, although the distribution has become more compact than that associated with the fragment $\mathrm{Z}$ range of $58 \leq \mathrm{Z} \leq 64$. It is also interesting to note an approximate symmetry of the pattern for IMF neutrons about the beam direction. Nevertheless, the IMF-neutron velocity distribution seems incompatible with the existence of a single, equilibrated source emitting neutrons isotropically. These data require the presence of at least two effective sources, presumably identical with the primary PLF and TLF. Overall, the two-dimensional velocity plots of Fig. IV.5c-f illustrate the evolution of dominantly binary reaction dynamics with increasing energy loss, i.e., decreasing impact parameter. How well this picture fits the data, and how complete it is, will become clear from quantitative comparisons between experimental and theoretical neutron spectra.

Such comparisons are made in Fig. IV.6 depicting a series of (one-dimensional) neutron velocity $\left(v_{n}\right)$ spectra of the Galilei-invariant form $d^{2} m / v_{n}^{2} d v_{n} d \Omega_{n}$ discussed in Section II.2 (cf Eq. II.3). These neutron spectra constitute a representative selection of 16 out of the 21 available distributions, taken at the in-plane neutron detector angles indicated in each panel. The neutrons were measured in coincidence with massive PLF's, detected at $\theta \approx-6.5^{\circ}$ with $\mathrm{Z}$ values in the range of $77 \leq \mathrm{Z} \leq 83$, a trigger condition selecting dominantly rather peripheral, quasi-elastic collisions. In Fig. IV.6, the data are given as solid dots, while solid curves represent fits with PLF and TLF as the only emitting sources. The dashed curves are calculated assuming a contribution by an additional third, non-equilibrium particle (NEP) source to the total neutron spectrum. 
Scanning the neutron spectra of Fig. IV.6 from small to large neutron angles $\theta_{n}$, one observes a characteristic change in shape of these spectra. In the forward direction, in particular at angles $\theta_{n}$ close to the fragment detection angle, e.g., at $\theta_{n}=-15.3^{\circ}, \theta_{n}=$ $28.4^{\circ}$, or $\theta_{n}=+18.5^{\circ}$, a well-defined component of neutrons is discernible, which has a maximum at a velocity close to that of the beam ( $\left.v_{\text {beam }}=7.5 \mathrm{~cm} / \mathrm{ns}\right)$. This component is attributed to sequential emission of neutrons from the PLF. Its TLF counterpart, a much slower neutron component, dominates the spectra at backward angles. The parameters of the PLF evaporative neutron component were obtained from a fit of Eq. II.3 to the neutron spectrum at $\theta_{n}=-15.3^{\circ}$, the PLF "master arigle". Those for the TLF source were determined from a similar fit to the 6 spectra taken at $\left|\theta_{n}\right|>140^{\circ}$, the TLF "master angles". The solid curves in Fig. IV.6 represent the superposition of both components, extrapolated to the various angles. The average neutron multiplicities, obtained from an extrapolation of the fitted spectra over the total solid angle, amount to $m_{P L F}=m_{T L F}=$ $m_{\text {evap }}=(2.3 \pm 0.4)$, whereas effective temperature parameters of $T_{P L F}=T_{T L F}=(1.6 \pm$ $0.2) \mathrm{MeV}$ result from this fit. Very reasonable values were also obtained for the kinetic energy loss $\left(E_{\text {loss }}=50 \mathrm{MeV}\right)$ and for the primary PLF emission angle $\left(\theta^{\prime}=-6.5^{\circ}\right)$.

It is clear from the comparison of the data with the solid curves in Fig. IV.6 that the overwhelming majority of the observed neutrons are produced in sequential evaporation by PLF and TLF. Nevertheless, the two-source fit is not quite perfect, and particularly noticeable discrepancies between experimental and theoretical spectra exist at intermediate angles of $40^{\circ}<\left|\theta_{n}\right|<90^{\circ}$, i.e., sidewards of the beam. Subtracting the PLF and TLF components, a weak experimental component remains, with an integrated multiplicity of $\mathrm{m}_{\mathrm{ex}} \approx(1.0 \pm 0.3), \quad$ approximately $18 \%$ of the total neutron multiplicity. The latter component can be described rather well by an additional effective moving source emitting isotropically a hard Maxwell-Boltzmann-type spectrum. This is demonstrated by a comparison of the data in Fig. IV.6 to the dashed curves containing this extra component. The non-equilibrium neutron component can be characterized by an effective source with a velocity coinciding with that of the c.m. system in the laboratory, a neutron multiplicity of $m_{N E P}=1.1$, and a temperature parameter of $\mathrm{T}_{\mathrm{NEP}}=7 \mathrm{MeV}$. The above combination of three effective sources leads to a very good representation of the experimental neutron distribution, at essentially all 21 angles.

For more dissipative collisions selected by PLF's of smaller $Z$ values, an NEP neutron component is no longer clearly discernible. Presumably, here this relatively weak component is masked by copious neutron emission from the hot PLF's and TLF's. The 
analysis of the neutron distributions measured under such conditions continued successfully to assume the presence of two dominant, PLF and TLF sources whose velocities are connected to the energy loss via two-body kinematics. The PIF and TLF "master angles" defined above were used to determine the total kinetic-energy loss. The corresponding source parameters, including the primary PLF emission angle, were subsequently obtained, using data at all angles. Because of the symmetry of the system, it seemed justified to perform a fit under the constraint of identical values for PLF and TLF source parameters. An estimation of systematical errors associated with this procedure yielded relative uncertainties of $10 \%$ for energy loss and total multiplicity of neutrons evaporated from either PLF or TLF, $20 \%$ for the effective temperature parameter, $4 \%$ for the total integrated multiplicity of all emitted neutrons, and an absolute uncertainty of $\Delta \theta^{\prime} \approx \pm 0.5^{\circ}$ for the primary PLF emission angle.

In Fig. IV.7, a collection of pertinent source parameters is given, as obtained from fits to the distributions of neutrons in coincidence with PLF's of an average atomic number indicated on the abscissa of the plot. The trends of some of these parameters with PLF Z have already been discussed in the previous section. For example, the bimodal distribution in total neutron multiplicity discussed earlier is observed in Fig. IV.7 to correspond to an analogous feature in the $\mathrm{Z}$ dependence of the kinematically determined energy loss $E_{\text {loss. }}$ Starting from the quasi-elastic fragments with $Z \approx 80$, this energy loss is observed to increase with decreasing fragment $Z$ value, until a first (local) maximum is reached for $P L F ' s$ with $Z \approx 60$, where $E_{\text {loss }} \approx(1.5 \pm 0.15) \mathrm{GeV}$. With further decreasing fragment $Z$, the dissipated energy seems to level off and even to decrease. This behavior is understood to be due to the contribution of nartially damped PLF fission fragments to the region with $\mathrm{Z} \approx 40$, as mentioned earlier. For comparison, the solid symbols in Fig. IV.7 are obtained from an analysis of spectra in coincidence with the fast component of PLF fission fragments, which should be relatively free of contamination by no-fission processes. The relatively low value of the total associated multiplicity for these fragments already suggests they are produced in rather peripheral collisions, which is supported by an associated energy loss (solid symbol) of only $E_{\text {loss }}=(0.6 \pm 0.2) \mathrm{GeV}$. The admixture of these fission fragments to fragments with the same $Z$ value produced in more dissipative collisions accounts for an overall energy loss of only $E_{\text {loss }}=(1.30 \pm 0.13) \mathrm{GeV}$ for $Z \approx 40$ fragments, a value that is unsystematically low.

Both the total multiplicity $\mathrm{m}_{\text {tot }}$ and the spectral temperature parameter $T_{\text {evap }}$ for $Z \approx 20$ IMF's have values very close to those deduced for the $Z \approx 60$ PLF's, an observation that 
suggests production in events with similar collision impact parameters and may even indicate a genetic relation between the two above fragment groups. However, the heavy IMF's contain less than half of the projectile mass, making a kinematical analysis of these incompletely measured events difficult and, at least, somewhat model dependent. Therefore, the energy loss of $E_{\text {loss }}=(1.90 \pm 0.19) \mathrm{GeV}$ determined for these IMF's kinematically from the neutron velocity distributions could be an overestimate. A better, less model-dependent estimate for the degree of dissipation associated with these IMF's is obtained from the measurement of the total neutron multiplicity $\left(\mathrm{m}_{\mathrm{evap}}=(27 \pm 2)\right.$ per fragment), indicating an energy loss of $E_{\text {loss }}=(1.50 \pm 0.15) \mathrm{GeV}$. Such an $E_{\text {loss }}$ value is consistent with the measured temperature parameter of $\mathrm{T}_{\text {evap }}=(3.7 \pm 0.4) \mathrm{MeV}$.

The $\mathrm{Z}$ dependence of the temperature parameter $\mathrm{T}_{\text {evap }}$ illustrated in Fig. IV.7 is easily understood, as it is closely connected with those of neutron multiplicity and kinetic energy loss. The small difference between $\mathrm{m}_{\text {tot }}$ and the sum of the multiplicities from PLF and TLF, $m=2 m_{\text {evap, }}$, points to a relatively weak dependence of the non-equilibrium neutron multiplicity with fragment $Z$ value. Of interest is the dependence of the primary PLF scattering angle $\theta^{\prime}$ on fragment $Z$ or energy loss shown in Fig. IV.7. It is observed in this figure that $\theta^{\prime}$ decreases approximately monots vith increasing energy loss, from the grazing angle to very forward angles, alth,. a kinematical fits of the data obtained for highly dissipative collisions are rather model-dependent. Such a behavior is consistent with the orbiting picture well-known [Sch84] from studies of low-energy reaction dynamics, it was also observed for another very heavy system, studied [Bal93, Sch93] in the present energy regime.

The data discussed above were taken in coincidence with fragments detected with the PLF1 trigger telescope, located at a forward angel of $\theta=-6.3^{\circ}$. One has to keep in mind that this telescope has a high efficiency for collecting the fast and forward-going PLF's and their evaporative products and may, hence, bias the data collected in favor of peripheral collisions. In order to explore, whether this kinematical selection influences the general results and conclusions, some data gathered in coincidence with the PLF3 telescope at $\theta$ $=-21.5^{\circ}$ will be discussed in the following. At this trigger angle, the dynamic range in atomic (mass) number of the fragments and, consequently, the range of impact parameters accessible to study, is limited. For example, only fragments with low and intermediate $Z$ values and kinetic energies appear in the spectrum taken at this angle. There are no distinguished groups of PLF's or PLF fission fragments, as is obvious from the raw data shown for this telescope in Fig. II.2. However, the neutron distributions 
have an appearance very similar to that of the patterns measured with the more forward fragment trigger angle. Even for MMF-neutron coincidences, one observes a bimodal neutron velocity distribution of the type shown in Fig. IV.6f.

An analysis of neutron emission patterns measured with a fragment trigger angle of $\theta=-$ $21.5^{\circ}$ is possible for the range of $6 \leq \mathrm{Z} \leq 50$. Parameters obtained from fits similar to those described above are shown in Fig. IV.8. For all fragment,, almost independently of their atomic numbers, the neutron velocity distributions are well reproduced by an energy loss of $\mathrm{E}_{\text {loss }}=(1.7-1.9) \mathrm{GeV}$, a multiplicity of evaporated neutrons of $\mathrm{m}_{\text {evap }} \approx(30 \pm 3)$, and an effective temperature parameter of $T_{\text {evap }} \approx(4.0 \pm 0.4) \mathrm{MeV}$. These parameter values appear to be quite consistent with what is observed at the more forward angles. At $\theta=-21.5^{\circ}$, however, there seems to be an increase in the total neutron multiplicity $\mathrm{m}_{\text {tot }}$ with increasing fragment $\mathrm{Z}$ value, in contrast to the nearly constant or declining multiplicity ( $m_{\text {evap }}$ ) and temperature parameter $\left(T_{\text {evap }}\right.$ ) of neutrons evaporated from PLF or TLF. This increase could be due to the NEP neutron component attributed previously to non-evaporative effects. The corresponding multiplicity is of the order of $\mathrm{m}_{\mathrm{NEP}}=\mathrm{m}_{\mathrm{tot}}$ $-2 m_{\text {evap }}=(5-10)$, for $Z$ values between 5 and 30 , but rises steeply to a maximum of $\mathrm{m}_{\mathrm{NEP}}=(25 \pm 5)$ at $Z \approx 50$. However, since $Z \approx 50$ fragments emitted at $|\theta| \approx 21.5^{\circ}$ are very rare and uncharacteristic events, and since the rise in overall multiplicity is not accompanied by a similarly drastic increase in temperature parameter, not too much significance can currently be attached to that number, without independent verification.

In order to explore the compatibility of different reaction models with the measured exclusive neutron emission patterns, the predicted shapes of these patterns were compared to the experimental data, as described further above for cases of relatively massive trigger fragments.

Particularly interesting is such a comparison to events associated with the emission of IMF's. In the literature, events of this nature are usually pictured as central collisions leading to the formation of a hot, expanding mononuclear composite system, in contrast with the intermediate dinuclear system invoked in most of the earlier discussion in this work. The intermediate system produced in the former case is assumed to contain most, if not all, of the energy of relative motion available in the entrance channel, which is subsequently released by evaporation of light particles and, in the final stages of the reaction, also by IMF's. This scenario should result in a nearly isotropic, very hard neutron emission pattern. As discussed qualitatively already in the context of Fig. IV.5, 
the data do not support such a scenario, however. In the following, this conclusion is redrawn quantitatively.

Fig. IV.9 depicts, for example, the one-dimensional Galilei-invariant spectra of neutrons coincident with IMF's with atomic numbers in the range of $5 \leq \mathrm{Z} \leq 19$, detected at $\theta=-$ 6.5 $5^{\circ}$. Data are given as solid dots, while the dashed curves represent the emission patterns consistent with a single (mononuclear) emitter and the solid curves correspond to emission from two effective sources. The simulation assuming a composite system underestimates the data both at forward and at backward angles, missing approximately $36 \%$ of the total neutron multiplicity. Overall, the two-source fit provides a better reproduction of the experimental emission pattern, except at some intermediate angles. Only $9 \%$ of the total multiplicity is missed in this latter approach. The statistically significant differences between these two fits are, unfortunately, not very clearly visible on the compressed logarithmic scale of Fig. IV.9. Evidence against the validity of a single-source scenario is provided by the results of a fit to these spectra with an isotropic emission pattern. Such a fit yields a total neutron multiplicity of only $\mathrm{m}_{C S}=45$ and a spectral temperature parameter of only $\mathrm{T}_{C S}=5 \mathrm{MeV}$. The values of both these parameters are clearly inconsistent with the total excitation energy of $2.9 \mathrm{GeV}$, expected for a composite system. Hence, it appears that the formation of a hot composite system or of an even hotter "participant zone" or "fireball" does not provide a realistic picture of a typical small-impact parameter collision, as identified by the emission of IMF's.

Neutron velocity distributions were also measured in coincidence with light charged particles, measured with the telescopes LCPI $\left(\theta_{\mathrm{lcp}} \approx-61.5^{\circ}\right)$ and LCP2 $\left(\theta_{\mathrm{lcp}} \approx-141.5^{\circ}\right)$. For IMF's ( $4 \leq Z \leq 13$ ), sufficiently accurate coincidence data were only gathered with the LCP1 telescope. The analysis of these data gave results in close agreement with those obtained for the $\theta=-21.5^{\circ}$ data. The velocity distributions of neutrons measured in coincidence with protons are found to be well described by a total multiplicity of $\mathrm{m}_{\mathrm{tot}} \approx$ 60 and an effective temperature parameter of $T_{\text {evap }} \approx 4 \mathrm{MeV}$. These fit values are the same for both detection angles. The neutron multiplicities in coincidence with protons are some (10-15)\% smaller than those associated with IMF's, which is not too surprising, in view of the very different selectivity of these two event triggers.

The above considerations suggest strongly that the basic reaction mechanism underlying the phenomenology of ${ }^{197} \mathrm{Au}+{ }^{208} \mathrm{~Pb}$ is a binary, dissipative process such as the wellknown nucleon exchange model NEM described in Section III. Because of its success in 
the description of energy and atomic-number distributions of fragments produced in partially damped ${ }^{197} \mathrm{Au}+{ }^{208} \mathrm{~Pb}$ collisions discussed in Section IV.1, it is interesting to study the compatibility of the NEM, augmented by evaporation calculations, with the important aspects of the experimental neutron emission patterns, for a larger class of events. Comparisons between source parameters deduced from experimental distributions and model predictions are presented in Figs. IV.10 and IV.11, depicting the energy-loss dependence of total experimental neutron multiplicity $\mathrm{m}_{\mathrm{tot}}$ and temperature parameters, $\mathrm{T}_{\mathrm{PLF}}$ and $\mathrm{T}_{\mathrm{TLF}}$, associated with the neutron energy spectra, respectively. Data for all event triggers considered in this work are included in these figures. The total multiplicity is defined here as the sum of evaporative and non-evaporative components, and the energy loss is derived kinematically from the fitted source velocities, assuming binary kinematics for the primary emitters. As is evident from these two figures, both the predicted multiplicities and the effective temperature parameters are reasonably well represented by the calculations, over the whole range of possible energy losses. Although these comparisons are not very detailed, several basic model assumptions are tested. The theoretical correlation $\mathrm{m}_{\mathrm{tot}}\left(\mathrm{E}_{\text {loss }}\right)$ relies on the assumption that the energy loss can be derived from binary kinematics and that the dissipated energy is converted entirely into intrinsic excitation energy of the reaction fragments. The subsequent conversion of excitation energy into multiplicity of neutrons and a prediction of the shape of their spectrum is effected by the statistical-model calculation.

Although the agreement between predicted and measured correlations is quite satisfactory, the NEM predicts full damping, $E_{\text {loss }}=\left(E_{\text {loss }}\right)_{\max }=2.35 \mathrm{GeV}$ to occur with measurable cross section. Experimentally, however, no condition was found selecting events corresponding to dissipated energies of more than $E_{\text {loss }}=1.9 \mathrm{GeV}$. A conclusion of great practical concern is that the total multiplicity of neutrons emitted in a reaction correlates very well with the total kinetic energy loss, a quantity which has played a very important role in the investigation of low-energy reaction dynamics.

\section{IV.4. Non-Equilibrium Neutron Emission}

As discussed already in the context of Fig.IV.6, there is evidence for neutron emission in processes other than statistical evaporation from the PLF and TLF from the present ${ }^{197} \mathrm{Au}+{ }^{208} \mathrm{~Pb}$ reaction. For peripheral collisions, the corresponding component is clearly discernible at sideward angles as an intensity of neutrons in excess of the theoretical evaporation spectra. Because of their hard spectrum and angular distribution that is 
uncorrelated with PLF or TLF, these neutrons are regarded as having been emitted in non-evaporative, or non-equilibrium (NEP) processes. For more dissipative collisions, this component is not unambiguously separable from the hard-neutron component from the PLF. This latter property makes an evaluation of the evolution of this non-evaporative emission process difficult to follow experimentally. Nevertheless. the present data are sufficiently accurate to provide some information on the angular and energy distributions of these neutrons and on the development of the NEP process with degree of dissipation.

The fit procedures employed in the evaluation of the measured neutron emission patterns have been explained extensively in previous sections. Fig. IV.6 shows, for trigger fragments with $77 \leq Z \leq 83$ detected at $\theta \approx-6.5^{\circ}$, fits to experimental neutron spectra obtained with three sources, PLF, TLF, and NEP, represented by the dashed curves. Here, the NEP process was assumed to be represented by a single effective source moving with the c.m. velocity and emitting neutrons, isotropically in its rest frame, and with a Maxwell-Boltzmann-type spectrum. The resulting average NEP-neutron multiplicity was found to be equal to $\mathrm{m}_{\mathrm{NEP}}=(1.0 \pm 0.4)$, for the effective spectral temperature parameter, a value of $\mathrm{T}_{\mathrm{NEP}}=(7 \pm 2) \mathrm{MeV}$ was obtained from the fit.

It is interesting to compare these numbers with low-energy systematics of fusion [Hil84] and damped [Wil89] reactions, extrapolated to the parameter values of the present ${ }^{197} \mathrm{Au}+{ }^{208} \mathrm{~Pb}$ system. For damped reactions with energies up to $\mathrm{E} / \mathrm{A}=16 \mathrm{MeV}$, the average NEP neutron multiplicity was found to be well described by [Wil89],

$$
\mathrm{m}_{\mathrm{NEP}} \approx 0.217\left(\mathrm{E}_{\mathrm{rad}}-2 \mathrm{MeV}\right) / \mu
$$

where $E_{r a d}=\left(E-V_{c}\right)_{r a d}$ is the radial kinetic energy at the interaction barrier. To obtain an estimate of the temperature parameter for NEP neutrons emitted in a peripheral heavyion collision from the fusion systematics [Hil88] applicable to central collisions,

$$
\mathrm{T}_{\mathrm{NEP}}(\mathrm{E}) \approx 1.7 \mathrm{MeV}+0.44\left(\mathrm{E}-\mathrm{V}_{\mathrm{c}}\right) / \mu
$$

one should probably substitute, in Eq. IV.2, the radial energy $E_{\text {rad }}$ for the total kinetic energy $E-V_{c}$ above the barrier. Applying Eqs. IV.I and 2 to the $E_{\text {loss }}=50-\mathrm{MeV}$, ${ }^{197} \mathrm{Au}+{ }^{208} \mathrm{~Pb}$ collision illustrated in Fig. IV.6, one predicts systematical values of $\mathrm{m}_{\mathrm{NEP}} \approx$ 1.8 and $\mathrm{T}_{\mathrm{NEP}} \approx 5.4$. These estimates are definitely of the order of the experimental values, the former being only two standard deviations larger than observed, the latter 
agreeing with its experimental counterpart within a single error bar. If confirmed, such an extension of the range of validity of the systematics, to Fermi energies and very heavy systems, would be highly significant for the prospects of generating critically hot nuclear systems in heavy-ion collisions at intermediate energies.

For more dissipative collisions, an estimation of the NEP neutron multiplicities becomes intrinsically more difficult and model-dependent. Nevertheless, it is possible to establish the basic trends of these multiplicities, for coincident fragment data taken at $\theta \approx-6.5^{\circ}$ and different trigger conditions. In Fig. IV.12, a quantity $m_{e x}$, related to the NEP multiplicity $m_{N E P}$ is plotted vs. the energy loss associated with the various event triggers identified in the legend of this figure. The multiplicity $m_{e x}=m_{e x}\left(\theta \approx-6.5^{\circ}\right)$ is obtained from the difference between the total experimental neutron velocity distributions and the PLF and TLF components. There is a general trend visible in the data of Fig. IV.12, showing a moderate increase of the multiplicity $\mathrm{m}_{\mathrm{ex}}$ with energy loss, although there is a large scatter in the experimental values. Multiplicity values derived for coincident fragments with $26 \leq Z \leq 58$, corresponding to event classes of different degrees of dissipation, as well as those obtained for IMF's, show NEP multiplicities $\left(\mathrm{m}_{\mathrm{ex}}\right)$ that are somewhat below the values corresponding to PLF's. The solid curve in Fig. IV.12 represents calculations with the Fermi-jet model [Ran87] discussed in Section III. It is apparently well consistent with the experimental data.

The performance of the latter Fermi jet model in the description of the data is detailed in Fig. IV.13 exhibiting some of the pertinent velocity spectra of neutrons associated with PLF's $(77 \leq Z \leq 83)$ produced in peripheral collisions and detected at $\theta \approx-6.5^{\circ}$. Data are indicated by symbols, while the curves represent different theoretical spectra. The sums of evaporative PLF and TLF neutron components are shown as heavy solid curves in Fig. IV.13. These evaporative spectra underestimate the high-velocity neutron components quite significantly, for several of the angles represented in Fig. IV.12 for the harder parts of the neutron spectra. Thinner solid lines and dashed curves illustrate the predictions of the Fermi jet model, for effective angular momenta of $l_{\text {eff }} \approx 1.6 \mathrm{k} \hbar$ and $l_{\text {eff }} \approx 1.5 \mathrm{k} \hbar$, respectively. The lower of these two $l_{\text {eff }}$ values corresponds to an energy loss of $\mathrm{E}_{\text {loss }} \approx$ $50 \mathrm{MeV}$, according to the model calculations. However, predictions for the Fermi jet spectra associated for this $l_{\text {eff }}$ value (thin solid curves) are seen to underestimate the experimental spectra at high velocities. The calculations for the lower angular momentum of $l_{\text {eff }} \approx 1.6 \mathrm{k} \hbar$, associated with higher energy loss, are seen to provide a better reproduction of the neutron spectra. Hence, one observes only a rather qualitative 
agreement between Fermi jet model and experimental data for the reaction ${ }^{197} \mathrm{Au}+{ }^{208} \mathrm{~Pb}$. The origin of the above considerable inconsistency between predicted Fermi jet intensity and the experimental high-velocity neutron components is presently not fully understood, but this discrepancy represents a serious challenge to the model.

\section{Conclusions}

The system ${ }^{197} \mathrm{Au}+{ }^{208} \mathrm{~Pb}$ studied in this work at $\mathrm{E} / \mathrm{A}=29 \mathrm{MeV}$ is one of the very few, very heavy reaction systems, investigated thus far at bombarding energies approaching the nucleonic Fermi energy. The observed reaction phenomenology for the ${ }^{197} \mathrm{Au}+{ }^{208} \mathrm{~Pb}$ reaction is markedly different from the features exhibited by lighter-ion reactions. Although the bombarding energy is sufficiently high to lead to a disassembly of a considerable part of the system, the reaction dynamics is dominantly of a binary, dissipative nature. Unlike for lighter reaction systems, no fusion or fusion-like processes, leading to an intermediate mononuclear composite, have been observed for the present reaction ${ }^{197} \mathrm{Au}+{ }^{208} \mathrm{Ph}$ This iatier reaction is clearly describable in terms of a two-stage process, for the entire range of impact parameters: the primary reaction producing a pair of massive, excited PLF and TLF, accompanied perhaps by a small number of promptly emitted NEP particles, and succeeded by the statistical decay of the massive fragments. While an essentially binary mechanism does not come unexpected for peripheral collisions, such a process is observed in the reaction ${ }^{197} \mathrm{Au}+{ }^{208} \mathrm{~Pb}$ for all trigger conditions, i.e., for essentially all impact parameters. This observation corroborates conclusions drawn from the contemporary study of another heavy system $\left({ }^{209} \mathrm{Bi}+{ }^{136} \mathrm{Xe}\right)$.

For rather peripheral, partially damped events ( $E_{\text {loss }} \leq 350 \mathrm{MeV}$ ), the binary reaction scenario has been demonstrated for the reaction ${ }^{197} \mathrm{Au}+{ }^{208} \mathrm{~Pb}$ directly by a kinematicalcoincidence measurement. These measurements also provide good estimates of the e: citation energies of the primary fragments, which turn out to be $E_{P L F}^{*} \approx E_{7 L F}^{*} \approx E_{l o s s} / 2$. Such collisions are quantitati vely very well described by a damped or dissipative reaction mechanism, as modeled by the NEM. The experimental Z-E correlation measured at forward angles appears to be essentially a result of the depletion of the PLF distribution by fission. This observation rejects several possible reaction mechanisms; it also suggests a new and effective method to measure the widths of primary fragment $\mathrm{A}$ and $\mathrm{Z}$ distributions. As predicted by the NEM, this width increases with decreasing PLF energy or increasing energy loss. The experimental data are sensitive enough to discriminate between numerical 
results obtained with NEM calculations carried out in different approximations. Interestingly, the data favor the sudden over the adiabatic limit. However, detailed comparisons between data and simulations have been possible so far only for a limited range of quasi-elastic and partially damped events, due to insufficient capability of evaporation models to describe the decay of highly excited nuclei.

Conclusions about dominantly binary, dissipative collision dynamics for all event triggers, were derived from a comparison of exclusive, Galilei-invariant neutron emission patterns with simulation calculations assuming various reaction scenarios. For all event triggers, these emission patterns are essentially bimodal and characteristic of sequential neutron emission from primary PLF and TLF, with only a small contribution by prompt, nonequilibrium processes. Deduced values of source velocities, neutron multiplicities, and spectral temperature parameters are found to be consistent with a dissipation of the total available energy of not more than approximately $E_{\text {loss }}=1.9 \mathrm{GeV}$. No conditions were discovered in which the entire available energy of $2.9 \mathrm{GeV}$, or even the entire kinetic energy of $2.35 \mathrm{GeV}$ above the interaction barrier was dissipated in a collision. It appears that fully damped final fragments, a hot composite system, or an even hotter "participant zone" or "fireball" are not realistic pictures of ${ }^{197} \mathrm{Au}+{ }^{208} \mathrm{~Pb}$ collisions.

Although a non-equilibrium neutron component is also observed in the data, most clearly in peripheral collisions, their influence on the balance of energy is not very important at the present bombarding energy. One observes only a rather qualitative agreement between Fermi jet model and experimental NEP data for the reaction ${ }^{197} \mathrm{Au}+{ }^{208} \mathrm{~Pb}$. The origin of the considerable inconsistencies between predicted Fermi jet intensities and the experimental high-velocity neutron yields is presently not fully understood, but this discrepancy represents a serious challenge to the model. Multiplicity and spectral shape of these NEP neutrons appear to be roughly consistent with the systematics of low-energy damped and fusion reactions. If confirmed, such an extension of the range of validity of the systematics, to Fermi energies and very heavy systems, would be highly significant for the prospects of generating critically hot nuclear systems in heavy-ion collisions at intermediate energies.

The new experimental data obtained from the present reaction ${ }^{197} \mathrm{Au}+{ }^{208} \mathrm{~Pb}$, together with rather similar recent observations made in the case of the ${ }^{209} \mathrm{Bi}+{ }^{136} \mathrm{Xe}$ reaction, seem to have opened up a new access to heavy-ion reaction mechanisms at intermediate energies. The unexpected results obtained in these studies challenge the current views of 
these mechanisms and promise to lend insight into previously unknown behavior of nuclear matter in the Fermi energy domain.

This work was supported by the U.S. Department of Energy Grant DE-FG0288ER40414. 


\section{References}

Ayi87 Ayi 87 S. Ayik, Phys. Rev. C35, 2086(1987)

Bar90 H.W. Barz et al., Phys. Let. B244, 161 (1990)

Ber88 G.F. Bertsch and S. Das Gupta, Phys. Rep. 160, 189 (1988), and references cited therein.

Bla75 M. Blann, Ann. Rev. Nucl. Sci. 25, 123 (1975)

Bla81 M. Blann, Phys. Rev. C23, 205 (1985)

Bla85 M. Blann, Phys. Rev. C31, 1245 (1985)

Blo 78 J.Blocki et al., Ann. Phys. (N.Y.) 113, 330 (1978)

Bon80 J.P. Bondorf et al., Nucl. Phys. A333, 285 (1980)

Bon85 J.P. Bondorf et al., Nucl. Phys. A443, 321 (1985), ibid. A444, 460 (1985)

Bon87 A. Bonasera et al., Nucl. Phys. A463, 653 (1987)

Bor90 B. Borderie et al., Ann. Phys. (Fr.) 15, 287 (1990)

Bow62H.R. Bowman et al., Phys. Rev. 126, 2120 (1962)

Bre92 S. Bresson et al., Phys. Lett. B294, 33 (1992)

Bro83 U. Brosa et al., Z. Physik A310, 177 (1983)

Bro89 U. Brosa et al., Nucl. Phys. A502, 423c (1989)

Bro90 U. Brosa et al., Phys. Rep. 197, 167 (1990)

Bud88 C. Budtz-Jorgenson and H.H. Knitter, Nucl. Phys. A490, 307 (1988)

Cec79 R.A. Cecil et al., Nucl Instr. Meth. 161, 439 (1979)

Day86 R. Dayras et al., Nucl. Phys. A460, 299 (1986).

Dos85 T. Dössing et al., Nucl. Phys. A327, 490 (1979); ibid. A433, 280 (1985)

Fri83 W.A. Friedman, Phys. Rev. C27, 569 (1983); ibid. C28, 16 (1983)

Fri88 W.A. Friedman, Phys. Rev. Let. 60, 2125 (1988)

Fri90 W.A. Friedman, Phys. Rev. C42, 667 (1990)

Gal89 J.Galin, Proc. Symp. Nucl. Dyn. Nucl. Disassembly, (J.B. Natowitz, Editor),

World Scientific, Singapore 1989, p.320

Ge187 C.K. Gelbke and D.H. Boal, Progr. Part. Nucl. Phys. 19, 33 (1987), and referencis therein

Gol74 A.S. Goldhaber, Phys. Lett. B53, 306 (1974)

Gra88 O. Granier et al., Nucl. Phys. A481, 109 (1988)

Gre86 C. Gregoire and B. Tamain, Ann. Phys. Fr. 11, 323 (1986) and references cited therein. 
Gro86 D.H.E. Gross et al., Phys. Rev. Lett. 56, 1544 (1986)

D. H. E. Gross et al., Phys. Rev. Lett. 56, 1544 (1986),

Gro87 D.H.E. Gross et al., Nucl. Phys. A461, 641 (1987); ibid. A461, 668 (1987)

Gro93 D.H.E. Gross et al., Prog. Part. Nucl. Phys. 30, 155 (1993), and references cited therein.

Gue89 D. Guerreau, in Nuclear Matter and Heavy-Ion Collisions, (M. Soyer, H.

Flocard, B. Tamain, and M. Porneuf, editors) Plenum Publ. Corp., 1989, p. 187, (1989).

Hil 79 D. Hilscher, Proc. Spec. Meet., Preequ. Nucl. React., Semmering 1988, (B. Strohmaier, Editor), OECD, Paris 1988, NEANDC-245 'U', p.245 (1988)

Hil93 D. Hilscher and H. Rossner, Ann. Phys. (Fr.) 17, 471 (1993)

Hof76 H. Hofmann and P.J. Siemens, Nucl. Phys. A257, 165 (1976)

Hof77 H. Hofmann and P.J. Siemens, Nucl. Phys. A275, 464 (1977)

Kim92 Y.D. Kim et al., Preprint MSUCL-850 (1992)

Lev87 S. Levit and P. Bonche, Nucl. Phys. A462, 109 (1987)

Lop89 J. A. Lopez and J. Randrup, Nucl. Phys. A503, 183 (1989)

Lop90 J. A. Lopez and J. Randrup, Nucl. Phys. A512, 345 (1990)

Mor88 L.G. Moretto and G.J. Wozniak, Progr. Part. Nucl. Phys. 21, 401 (1988).

Mor92 L.G. Moretto et al., Phys. Rev. Let. 69, 1884 (1992)

Mou78 J.B. Moulton et al., Nucl Instr. Meth. 157, 325 (1978)

Nic92 N.G. Nicolis et al., Comp. Phys. Comm., in press

Pia91 E. Piasecki et al., Phys. Rev. Let. 66, 1291 (1991)

Que91 B.M. Quednau et al., Proc. VII Worksh. Nucl. Dyn., (W. Bauer and J. Kapusta, editors) World Scientific, Singapore 1991, 37

Que93 B.M. Quednau et al., Proc. VIII Worksh. Nucl. Dyn., (W. Bauer and B.Back, editors) World Scientific, Singapore 1993, 107

Que93a B.M. Quednau, Ph.D. Thesis, Ruhr Univ. Bochum, 1993, unpublished

Ran87 J. Randrup and R. Vandenbosch, Nucl. Phys. A474, 219 (1987)

Sch84 W.U. Schröder and J.R. Huizenga, in Treatise on Heavy-Ion Science, (D.A. Bromley, Editor), Plenum Press (New York and London, 1984), Vol.2, pp.113-726; and references cited therein

Sch87 W.U. Schröder et al., Nucl. Sci. Res. Conf. Ser.(Harwood) Vol. 11, 255 (1987)

Sch92 W.U. Schröder, Nucl. Phys. A539, 493c (1992)

Sur87 E. Suraud, Nucl. Phys. A462, 109 (1987) 
Sur 89 E. Suraud, Proc. Symp. Nucl. Dyn. Nucl. Disassembly, (J.B. Natowitz, editor) World Scientific, Singapore, 1989, p. 464.

Van73 R. Vandenbosch and J.R. Huizenga, Nuclear Fission, Academic Press (New York and London) 1973

Wil87 J.L.Wile et al., Phys. Rev. C35, 1608 (1997)

Zie85 J.F. Ziegler et al., The Stopping and Range of Ions in Solids, Pergamon (New York), 202 


\section{Tables}

Table1: Reaction parameters for ${ }^{197} \mathrm{Au}+{ }^{208} \mathrm{~Pb}$ at $\mathrm{E} / \mathrm{A}=29 \mathrm{MeV}$ as estimated from systematics

$\begin{array}{llrl}\text { Reduced Mass } & \mu & 101.2 & \\ \text { Strong-Absorption Radius } & R_{\mathrm{sa}} & 15.9 \mathrm{fm} \\ \text { Coulomb Energy } & \mathrm{V}_{\mathrm{c}} & 0.59 \mathrm{GeV} \\ \text { Beam Energy } & \mathrm{E}_{\mathrm{lah}} & 6.03 \mathrm{GeV} \\ \text { Beam Energy/Nucleon } & \mathrm{E}_{\mathrm{lah}} / \mathrm{A} & 29.0 \mathrm{MeV} \\ \text { c.m. Energy } & \mathrm{E}_{\mathrm{cm}} & 2.93 \mathrm{GeV} \\ \text { Available Energy } & \mathrm{E}_{\mathrm{cm}}-\mathrm{V}_{\mathrm{c}} & 2.35 \mathrm{GeV} \\ \text { Grazing Angle (Projectile) } & \theta_{1 / 4} \text { (Proj.) } & 6.3 & 0 \\ \text { Grazing Angle (Target) } & \theta_{1 / 4} \text { (Targ.) } & 83.5 & 0 \\ \text { c.m. Grazing Angle } & \theta_{1 / 4} \text { (c.m., Proj.) } & 16.0 & 0 \\ \text { Maximum Angular } & l_{\mathrm{max}} & 1.7 \mathrm{k} \hbar \\ \text { Momentum } & & & \\ \text { Reaction Cross Section } & \sigma_{\mathrm{R}} & 6.3 \mathrm{~b}\end{array}$


Table 2: Solid-state detector telescopes used in the experiment for projectile-like fragments (PLFi) or for light charged particles and intermediate-mass fragments $(\mathrm{LCPi})$.

Telescope

PLF1

PLF2

PLF3

LCP1

(1)

LCP2
Thickness ( $\mu \mathrm{m})$

47

300

4000

25

307

5000

35

312

5000

38

300

1000

5000

40

300

4000
Lab. Angle $\theta$

$-6.5^{0}$

$+8.50$

00

Lab. Angle $\phi$

00

00

$-21.50$

$-61.50$

$-18^{\circ}$

$-141.5^{0}$

$-130$ 


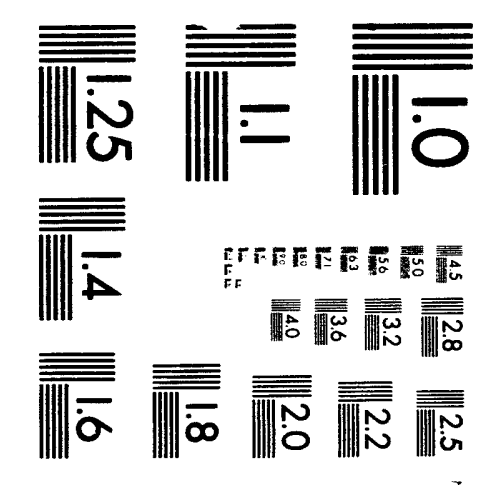



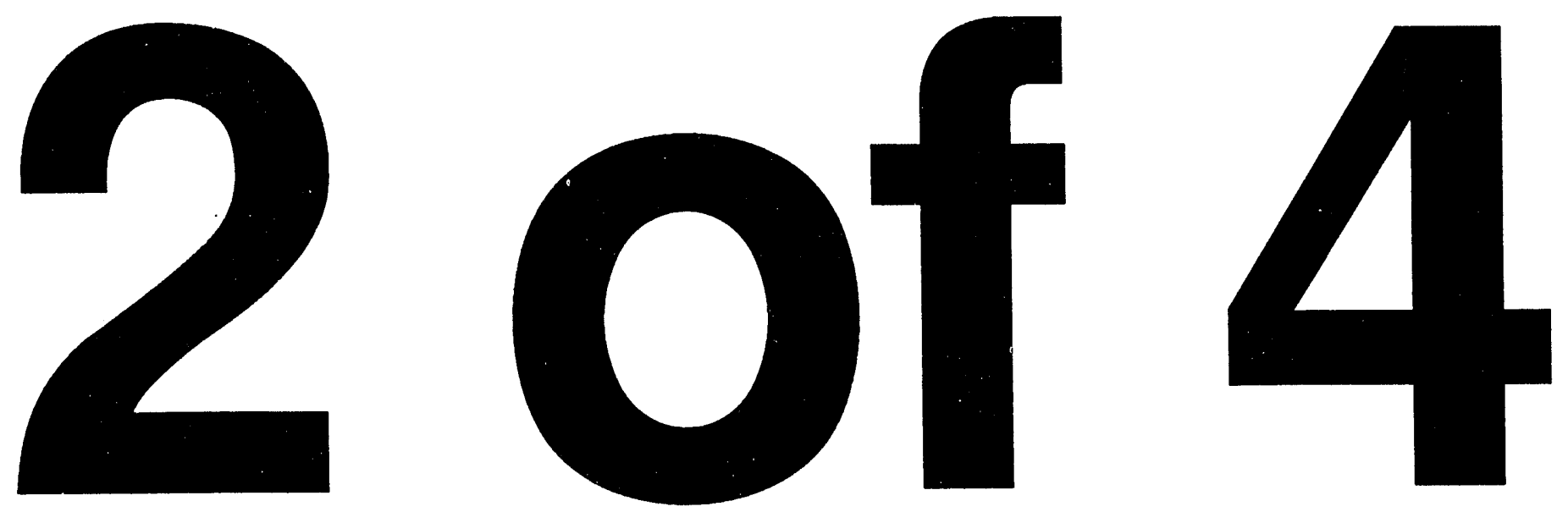


\section{Figure Captions}

Figure I.1: Schematics of the experimental setup, overyiew (top) and detail (bottom).

Figure I1.2: Correlation $\Delta \mathrm{E} 1-\Delta \mathrm{E} 2$ between the pulse heights, in arbitrary units, measured with the first two elements of telescope PLF3. The electronic $\Delta E 1$-threshold visible in the data excludes charged products with $\mathrm{Z} \leq 4$.

Figure 11.3: Raw TOF spectrum containing both neutrons and $\gamma$-rays, as obtained with a neutron detector at $\theta_{n} \approx 37.8^{\circ}$ (light histogram). The peak at small TOF is due to $\gamma$-rays, while the broad distribution on its right represent neutron events. The heavy histogram indicates the events identified as neutrons by the neutron- $\gamma$ pulse-shape discrimination circuitry. This latter spectrum shows a weak contamination by $\gamma$-ray events. Also indicated are the TOF corresponding to the neutron threshold applied, and the range used for background determination.

Figure 11.4: Identification matrices for recoil particles detected in a NE213 neutron scintillation detector. The scatter plots of pulse shape signal $\Delta S$ (a) or TOF (c) vs. light output were measured in anticoincidence with a thin plastic counter in front of the NE213 detector, the data shown in b) and d) were taken in coincidence with this counter. The various branches correspond to Compton recoil electrons (e), recoil protons ( $p$ ), a particles from the $(n, a)$ reaction on carbon, or the ridges corresponding to $p, d, t$, and $\alpha$ particles incident on the plastic/NE213 detector telescope.

Figure II.5: Results of the kinematical PLF-TLF coincidence measurement. The yield is plotted vs atomic number $\left(Z_{P L F}\right)$ of PLF's, in coincidence with TLF's measured at the indicated correlation angles $\theta_{\mathrm{TLF}}$. The arrow heads indicate the mean $\mathrm{Z}_{\mathrm{PLF}}$ values of the distributions, obtained from an average over $60 \leq \mathrm{Z}_{\mathrm{PLF}} \leq 90$.

Figure III.1: Contour diagram of the potential energy surface (PES) V(Z $Z_{\text {PLF }}, N_{P L F}$ ) for fragmentations of the system ${ }^{197} \mathrm{Au}+{ }^{208} \mathrm{~Pb}$, an angular momentum of $l=0$, and a separation distance of $r=R s a$, as calculated from the liquid-drop model with shell corrections. The numbers at the contours are given in units of MeV. The entrancechannel projectile-target combination ("injection point") is located in close proximity of the minimum ("symmetry point"). 
Figure II.2: Results of an NEM simulation for the dissipative $l=1.45 \mathrm{k} \hbar{ }^{197} \mathrm{Au}+{ }^{208} \mathrm{~Pb}$, $\mathrm{E} / \mathrm{A}=29 \mathrm{MeV}$, trajectory associated with an energy loss of $\mathrm{E}_{\text {diss }}=0.97 \mathrm{GeV}$. Plotted are the predicted time dependencies of dissipated energy (top panel), relative velocity (second from top), average fragment temperature (third from top), and of the characteristic neutron evaporation time (bottom). The dissipative interaction begins at a time of approximately $t=6 \cdot 10^{-22} \mathrm{~s}$. The vertical arrows indicate the time at which the classical turning point is reached.

Figure III.3: Time evolution of the relative distance R of PLF and TLF, and the geometrical and of the effective neck radii, $\mathrm{C}$ and Ceff, respectively (top), average nuclear temperature $\tau$ (center), and rate $n_{\text {proj }}$ (in units of $10^{22} \mathrm{~s}^{-1}$ ) of PLF neutrons emitted in a Fermi jet. For comparison, the classical turning point of the trajectory for the relative motion is reached in this collision at $t=1.8 \cdot 10^{-22} \mathrm{~s}$.

Figure IV.1: The experimental fragment yield measured with the PLFI telescope at $\theta \approx$ $-6.5^{\circ}$ is plotted as a two-dimensional contour diagram vs. atomic number $\mathrm{Z}$ and laboratory kinetic energy. The horizontal ridge at $Z \approx 82$ is due to slit scattering, a diagonal gap visible in the distribution for fragment $Z$-values between 8 and 40 is caused by a deadlayer in one of the elements of this telescope. The solid line along the ridge represents results of simulation calculations based on the NEM, with arrows illustrating the variation of the PLF excitation energy along the ridge line. The insert gives an illustration of the primary $\mathrm{Z}$ distribution prior (shaded Gaussian) and post (solid black Gaussian) fission.

Figure IV.2: The two-dimensional experimental correlation between atomic number and laboratory energy of fragments detected at $\theta \approx-6.5^{\circ}$ is coarsely outlined (cf. Fig. IV.1). The superimposed grid defines bins in fragment $Z$ and $E$, the number in each bin corresponds to the multiplicity $\mathrm{m}_{\text {tot }}$ of coincident neutrons, averaged over the bin.

Figure IV.3: Projections of the two-dimensional distribution $m_{t o t}(Z, E)$ shown in Fig. IV.2. The intensity-weighted average associated-neutron multiplicity, $\left\langle m_{n}\right\rangle$, is plotted vs. the average fragment atomic number, $\langle\mathrm{Z}\rangle$. Experimental data are represented by the histogram, while calculations carried out with the NEM are shown as solid dots.

Figure IV.4: Correlation between average neutron multiplicity, $\left\langle\mathrm{m}_{\mathrm{n}}\right\rangle$, and average measured laboratory kinetic energy, $<E>$, of the fragments detected at $\theta \approx-6.5^{\circ}$ ( histogram). The solid dots represent results of NEM simulation calculations. 
Figure IV.5: Linear, two-dimensional contour maps of the Galilei-invariant cross section $d^{2} \sigma / v_{n x} d v_{n x} v_{n z}$ of neutrons in coincidence with fragments from the reaction ${ }^{197} \mathrm{Au}+{ }^{208} \mathrm{~Pb}$ at $\mathrm{E}_{\mathrm{lab}} / \mathrm{A}=29 \mathrm{MeV}$, detected at $\theta \approx-6.5^{\circ}$. The cross sections are plotted $v s$. the neutron velocity components parallel $\left(v_{n x}\right)$ and perpendicular $\left(v_{n y}\right)$ to the beam. Panels a) and b) are theoretical distributions, while the distributions e) - f) are experimental, as measured for various conditions on the "trigger" fragments detected with the PLFI telescope. The arrows in Fig. IV.5a indicate the velocities of PLF, TLF, and of the c.m. system in the laboratory.

Figure IV.6: Representative selection of 16 out of the 21 available one-dimensional, Galilei-invariant neutron velocity $\left(v_{n}\right)$ spectra $d^{2} m / v_{n}^{2} d v_{n} d \Omega_{n}$. The differential multiplicity of neutrons in coincidence with massive PLF's is plotted $v s$. laboratory velocity $\left(v_{n}\right)$. The neutron detector angles are indicated in each panel. The data are given as solid dots, while solid curves represent two-source fits, with PLF and TLF as the only emitting sources. The dashed curves include a contribution by an additional third, non-equilibrium source.

Fig. IV.7: Dependence of neutron-emitting source parameters on the average Z-value (< $\mathrm{Z}>$ ) of coincident fragments detected at $\theta=-6.3^{\circ}$, as obtained from fits to the invariant velocity distributions of neutrons.

Fig. IV.8: Dependence of neutron-emitting source parameters on the average Z-value $(<$ $\mathrm{Z})$ of coincident fragments detected at $\theta=-21.5^{\circ}$, as obtained from fits to the invariant velocity distributions of neutrons.

Figure IV.9: One-dimensional Galilei-invariant spectra of neutrons coincident with IMF's with atomic numbers in the range of $5 \leq \mathrm{Z} \leq 19$, detected at $\theta=-6.5^{\circ}$. Data (solid dots) are compared with emission patterns calculated for a single (dashed curve) or two (solid curve) effective sources of neutrons.

Figure IV.10: Comparisons between source parameters deduced from experimental distributions (symbols) and model predictions (curve). The total neutron multiplicity $\mathrm{m}_{\text {tot }}$ is plotted $v s$. energy loss derived from fits of the neutron emission patterns associated with various trigger fragments indicated in the legend. 
Figure IV.11: Comparisons between source parameters deduced from experimental distributions (symbols) and model predictions (curve). The temperature parameters, $\mathrm{T}_{\mathrm{PLF}}$ and $\mathrm{T}_{\mathrm{TLF}}$, associated with the neutron energy spectra, are plotted $v s$. energy loss derived from fits of the neutron emission patterns associated with various trigger fragments indicated in the legend.

Figure IV.12: The quantity $m_{e x}$, related to the NEP multiplicity $m_{N E P}$, is plotted vs. the energy loss associated with the various event triggers identified in the legend. The solid curve represents calculations with the Fermi-jet model [Ran87].

Figure IV.13: Velocity spectra of neutrons associated with PLF's $(77 \leq \mathrm{Z} \leq 83)$ produced in peripheral collisions and detected at $\theta \approx-6.5^{\circ}$. The sums of evaporative PLF and TLF neutron components are shown as heavy solid curves. Thinner solid lines and dashed curves illustrate the predictions of the Fermi jet model, for effective angular momenta of $l_{\text {eff }} \approx 1.6 \mathrm{k} \hbar$ and $l_{\text {eff }} \approx 1.5 \mathrm{k} \hbar$, respectively. 


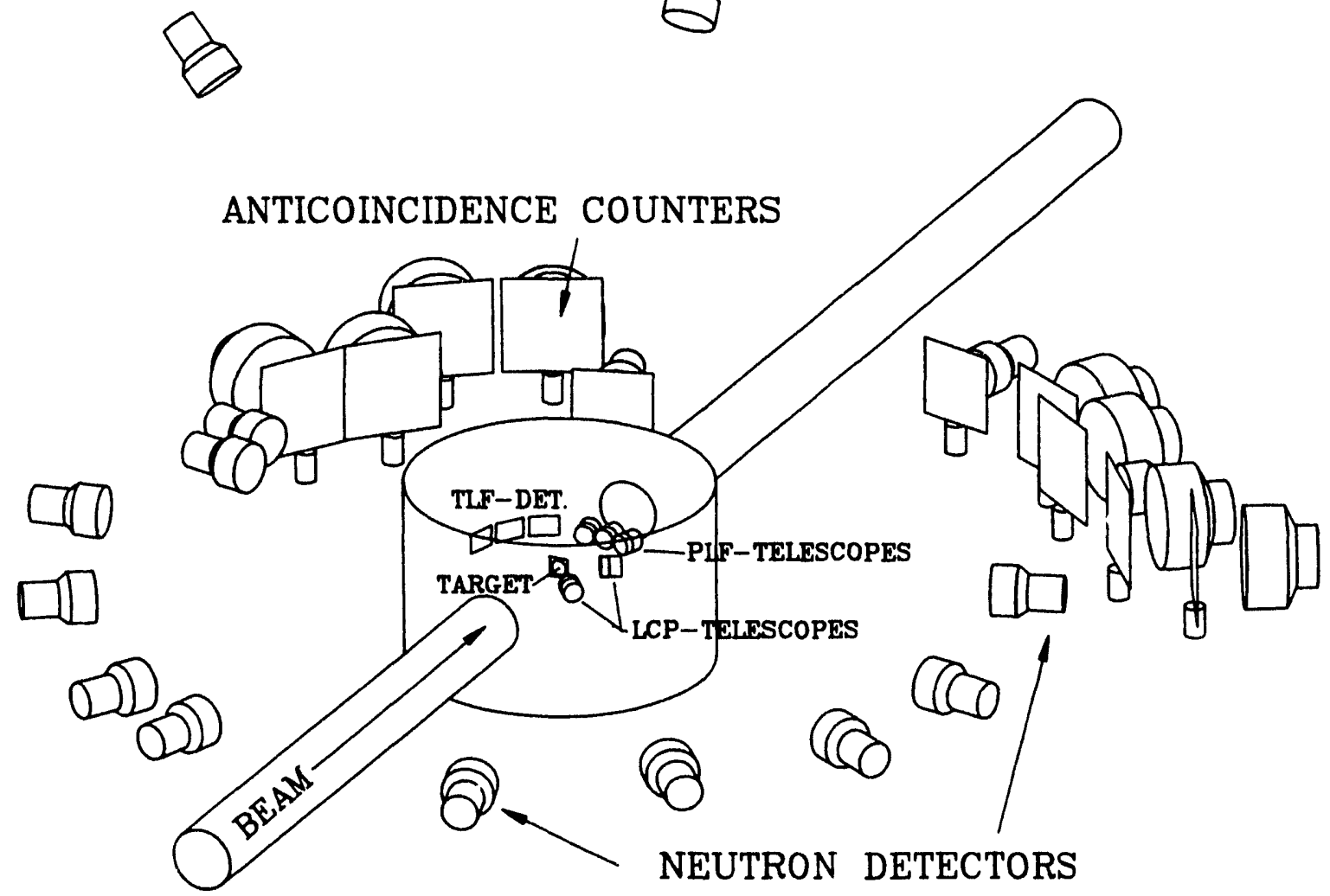

$\theta$

Figure II.1A

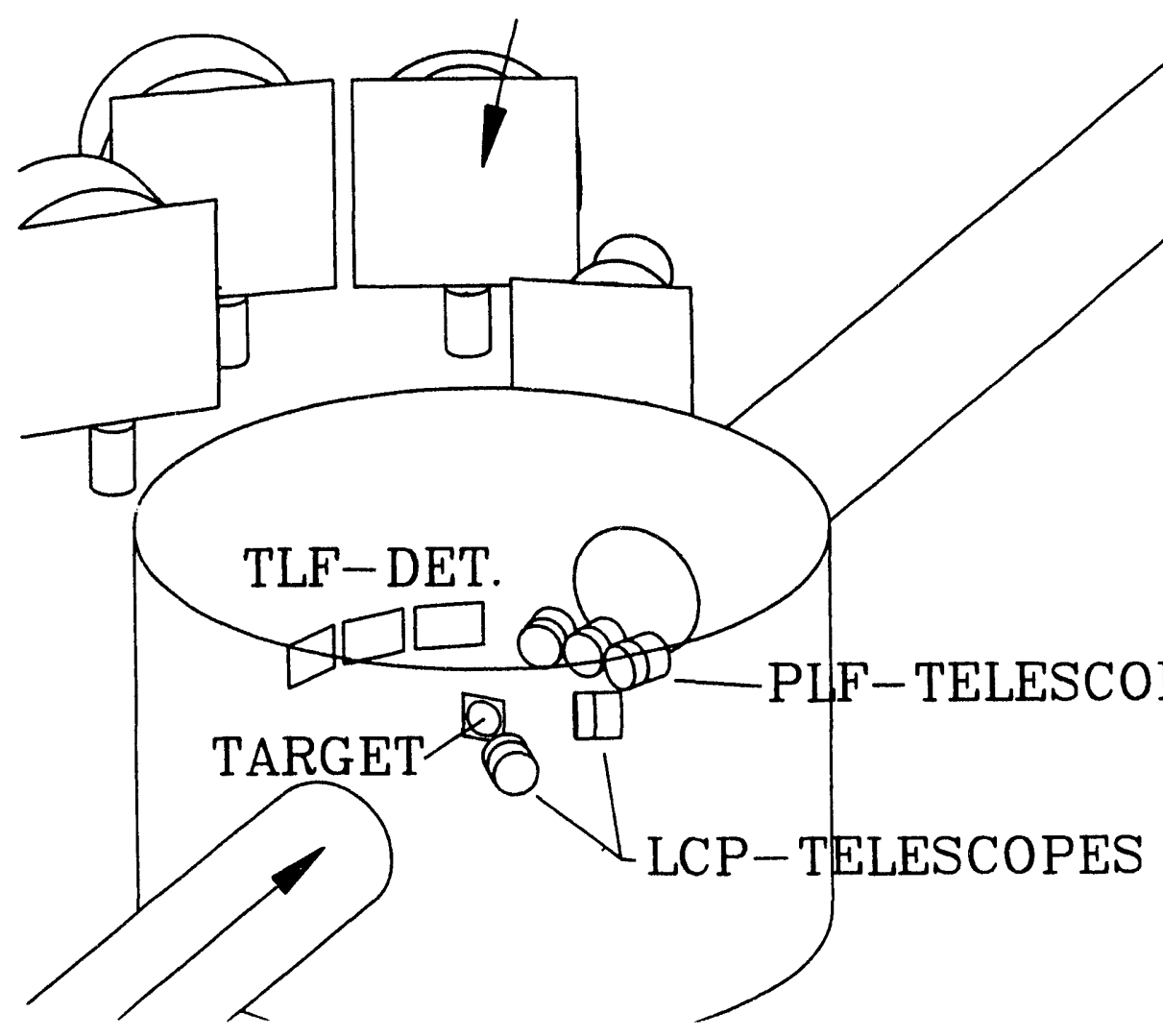

Figure II.1B 


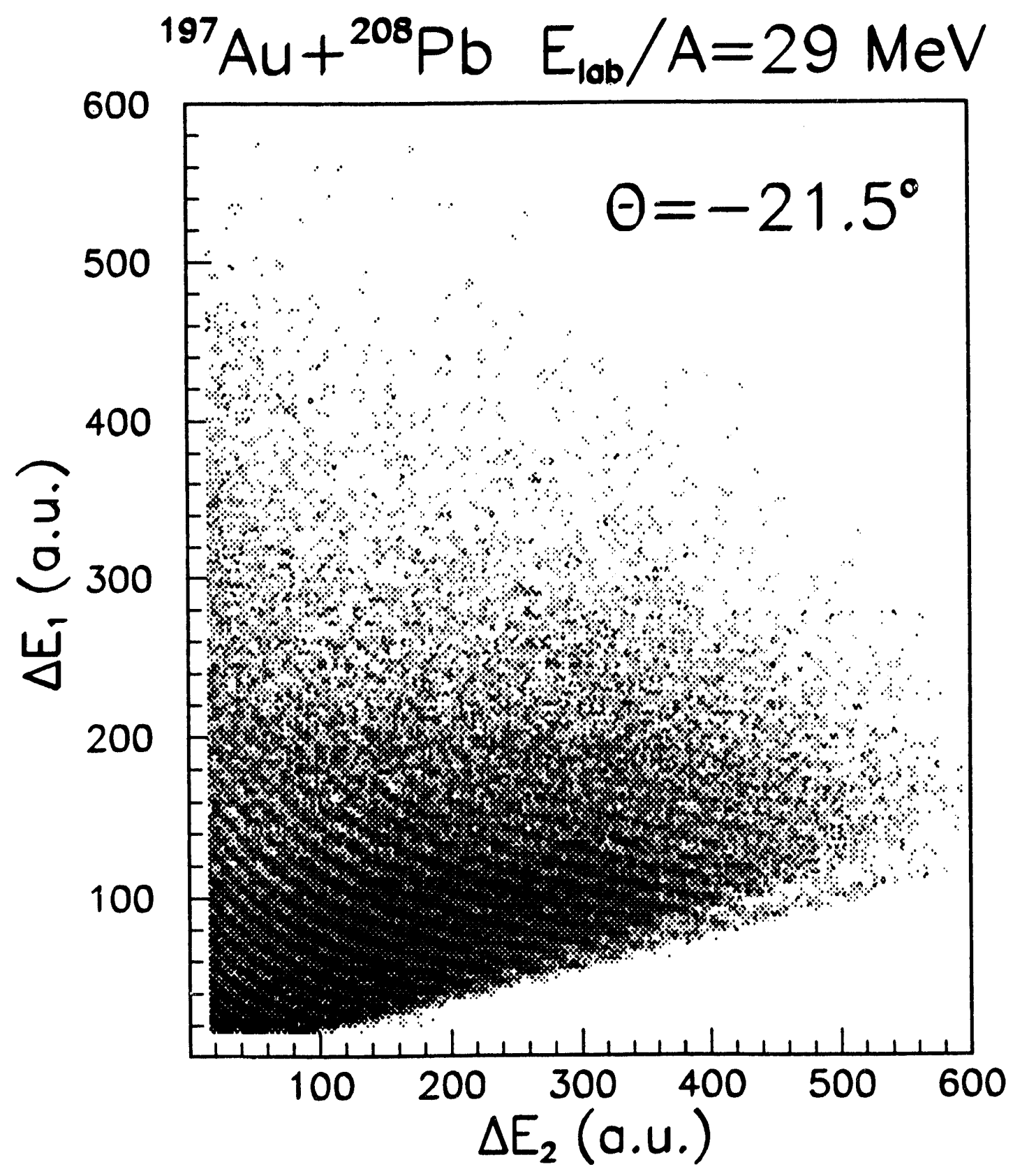

Figure II. 2 


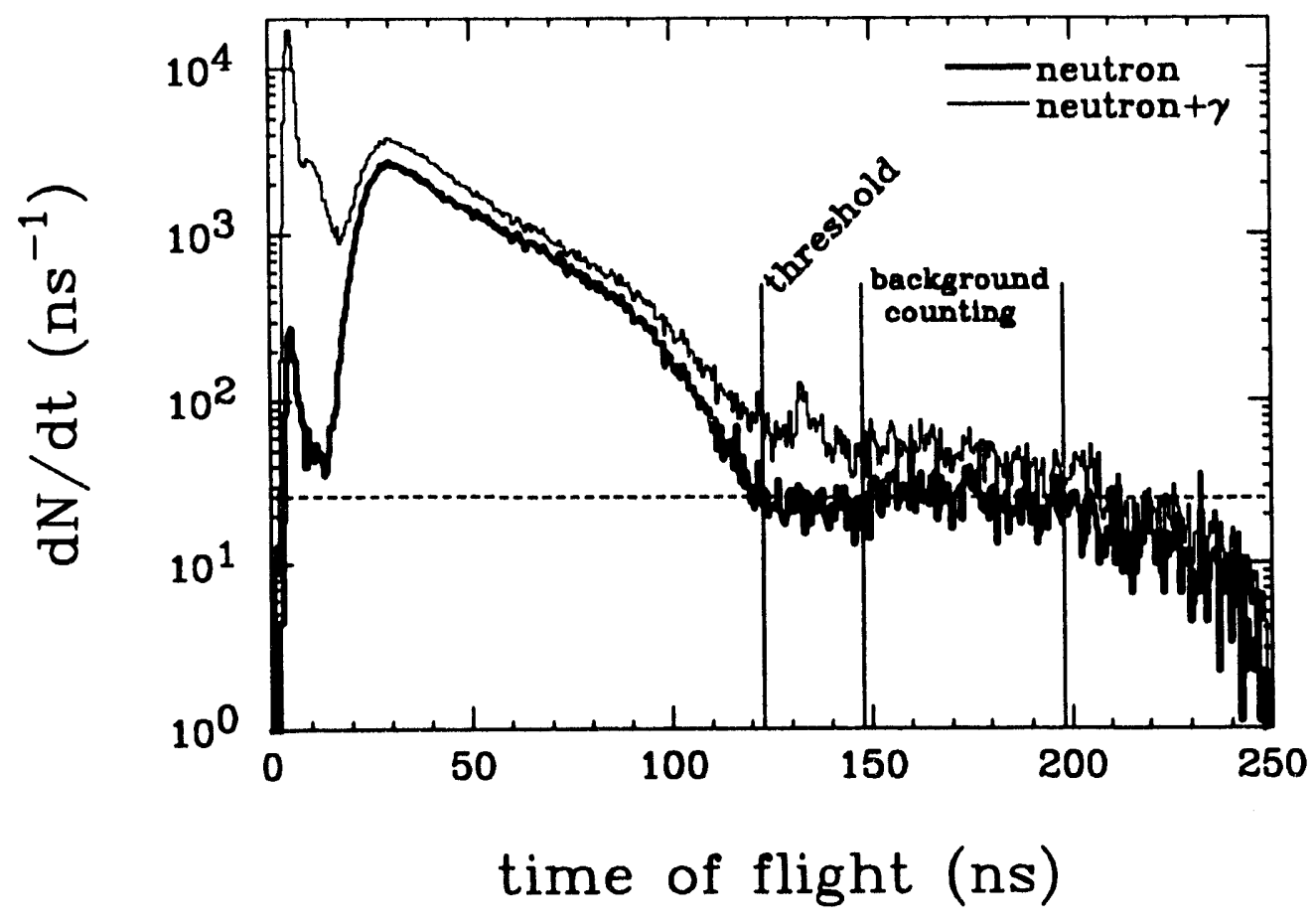

Figure II.3 


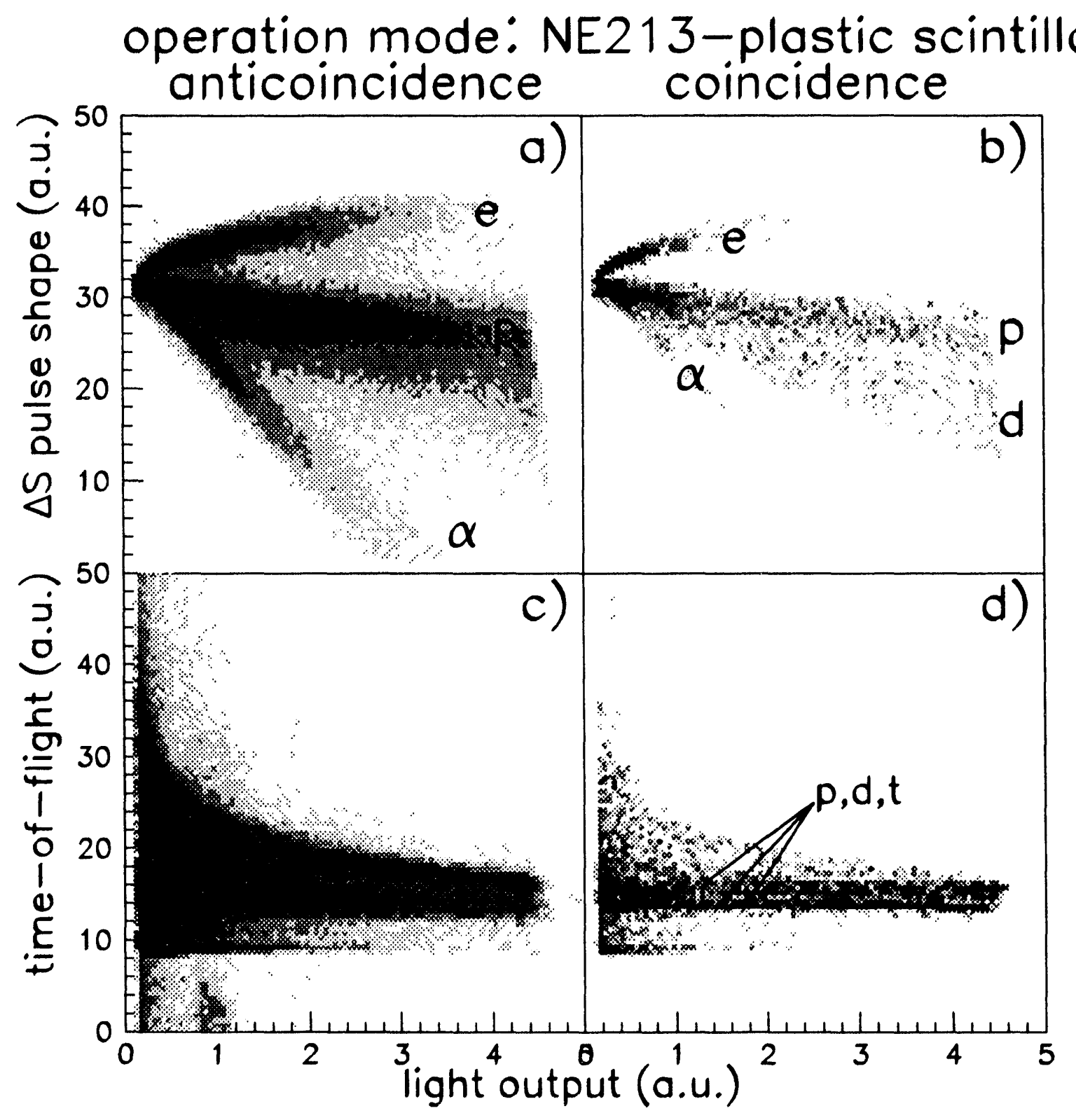

Figure II.4 


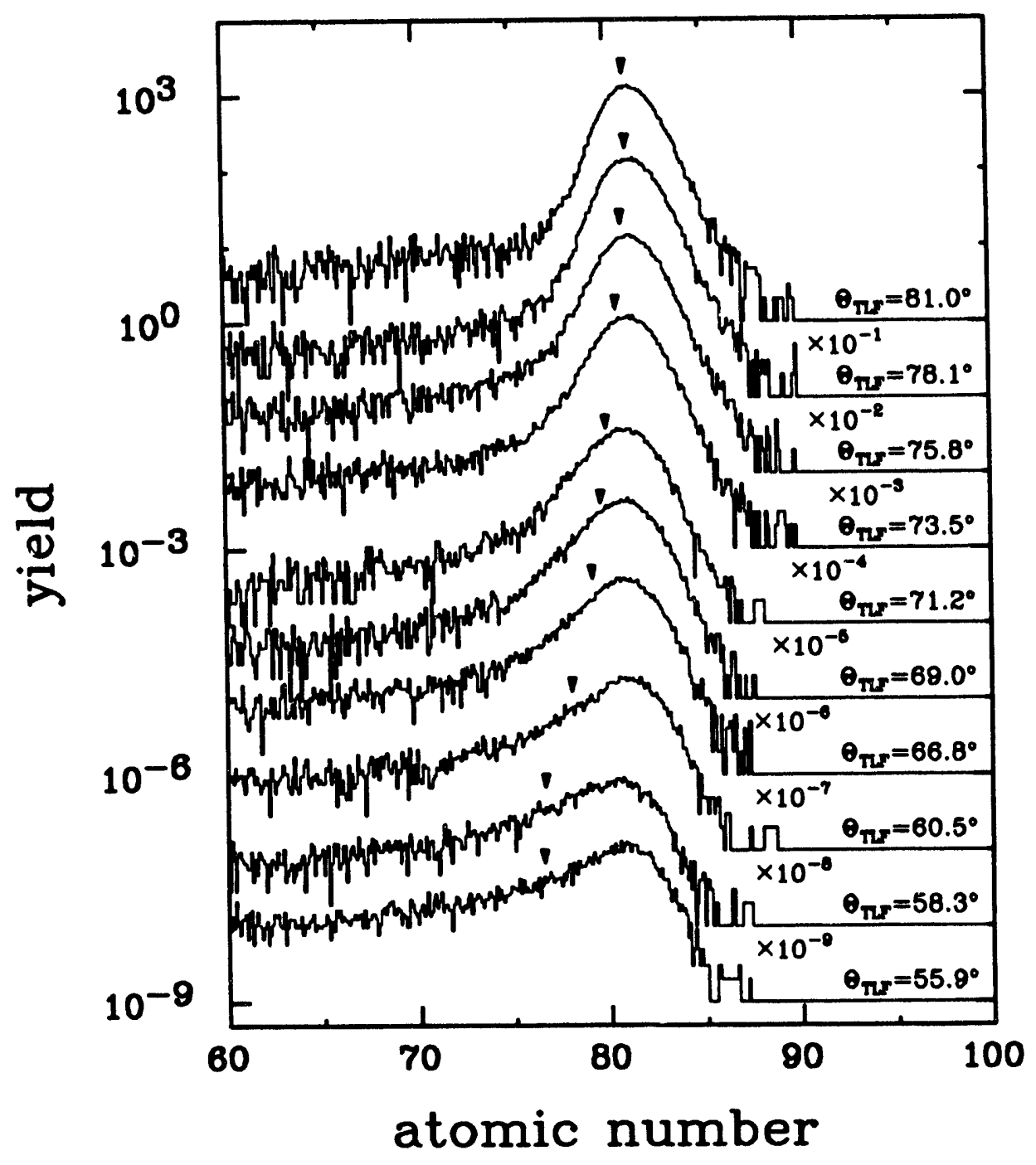

Figure II.5 


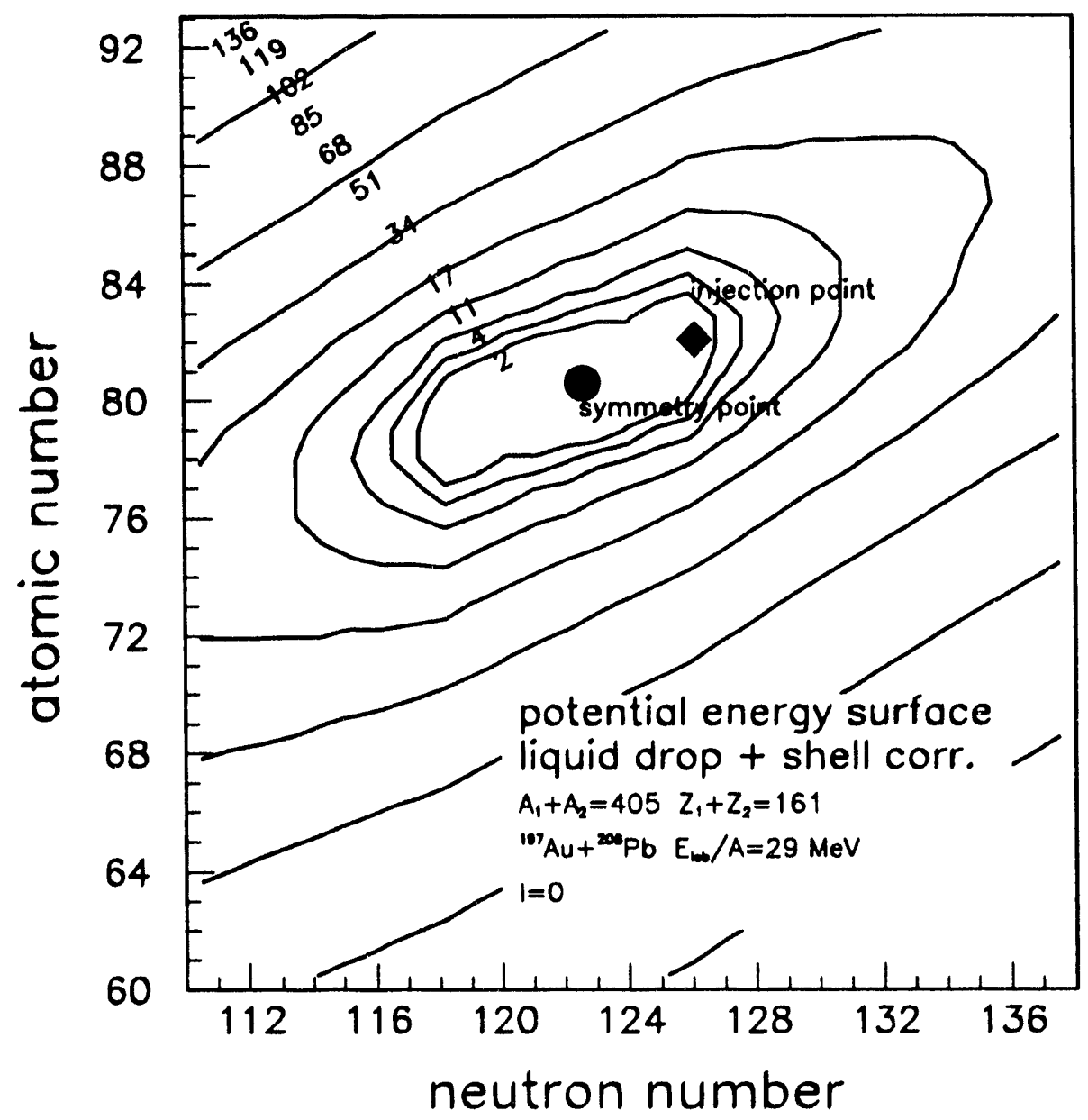

Figure III.1 
${ }^{197} \mathrm{Au}+{ }^{208} \mathrm{~Pb} \quad \mathrm{E} / \mathrm{A}=29 \mathrm{MeV} /$ nucleon Strongly Damped Collisions

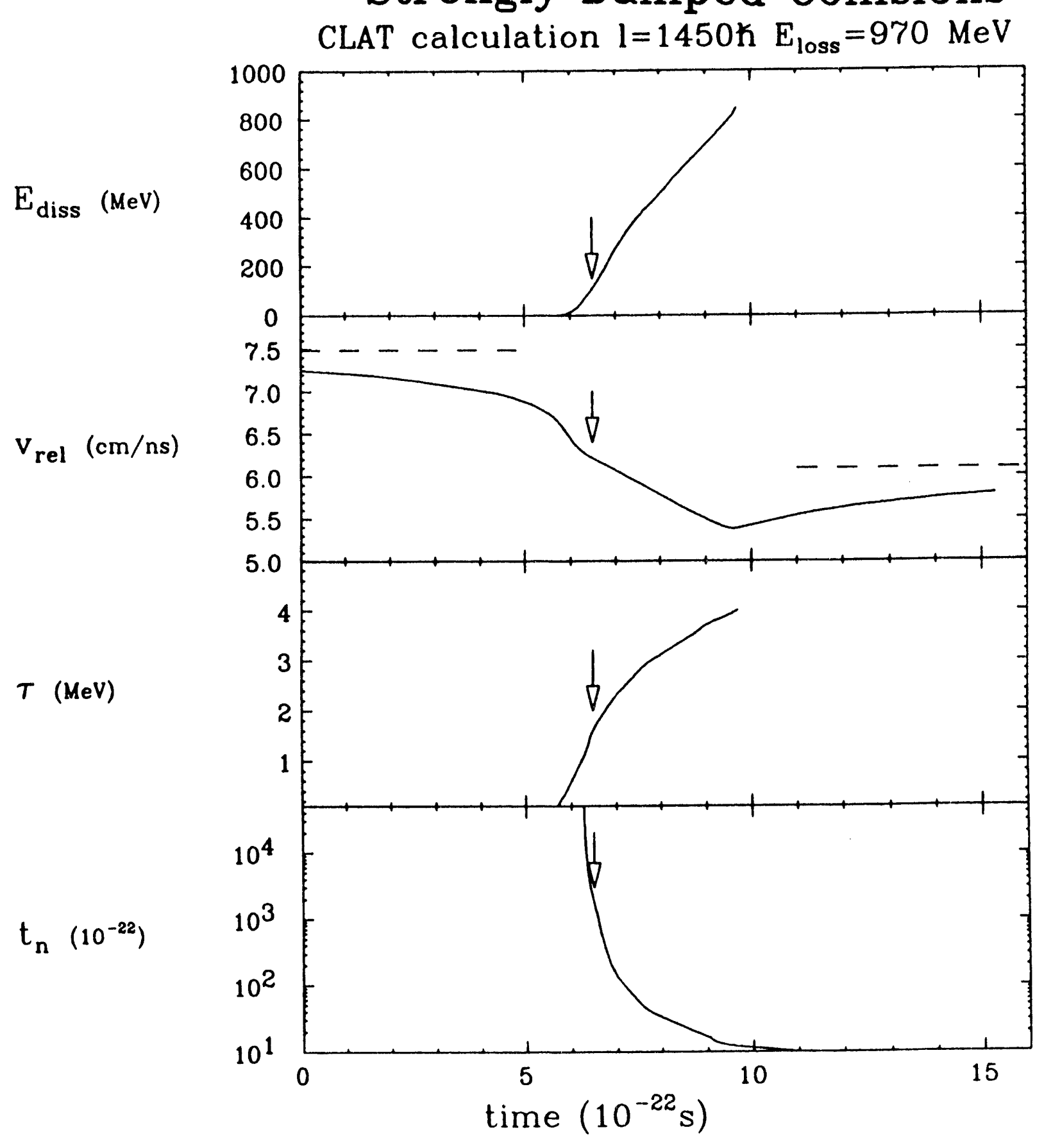

Figure III.2 


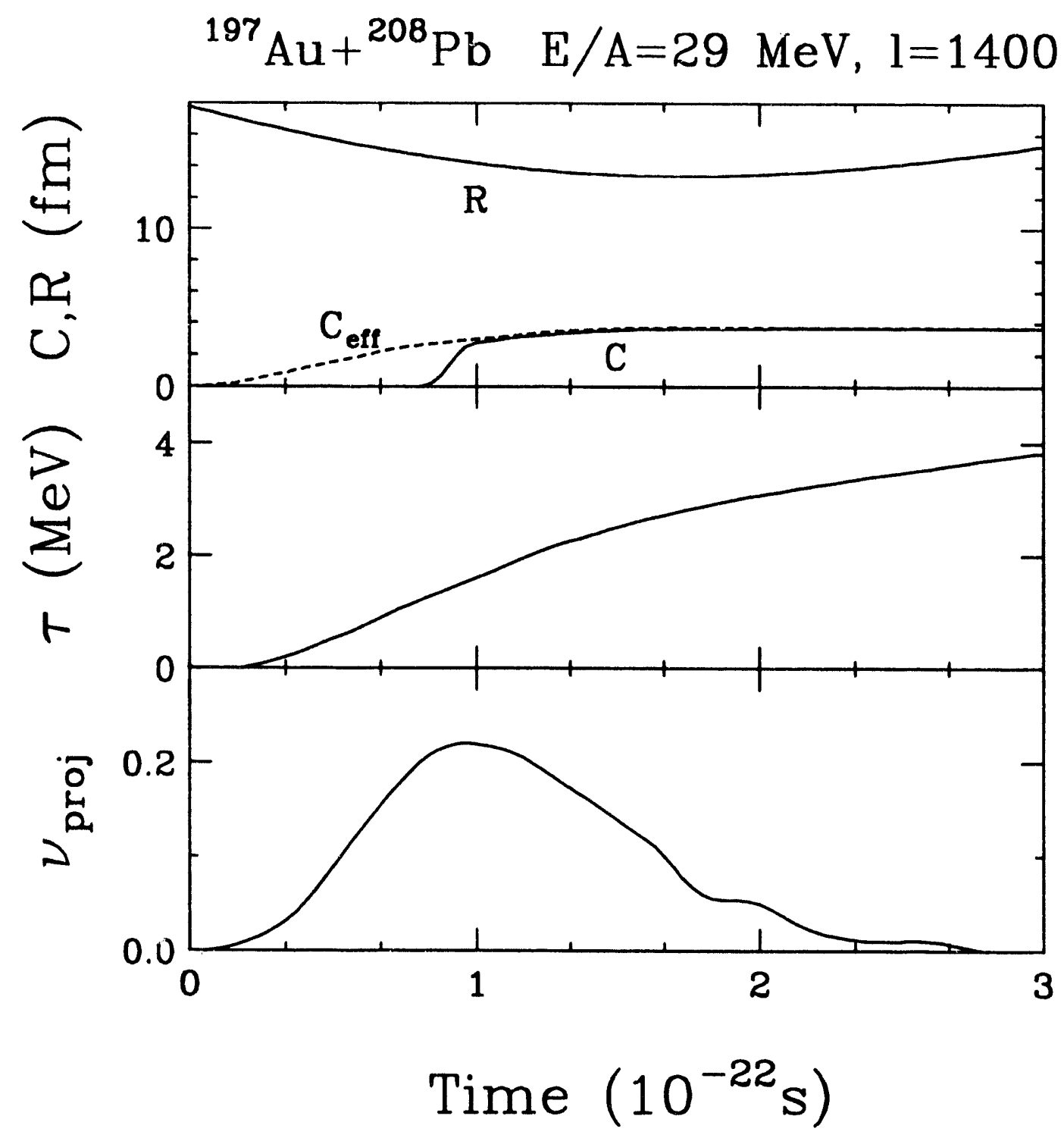

Figure III.3 


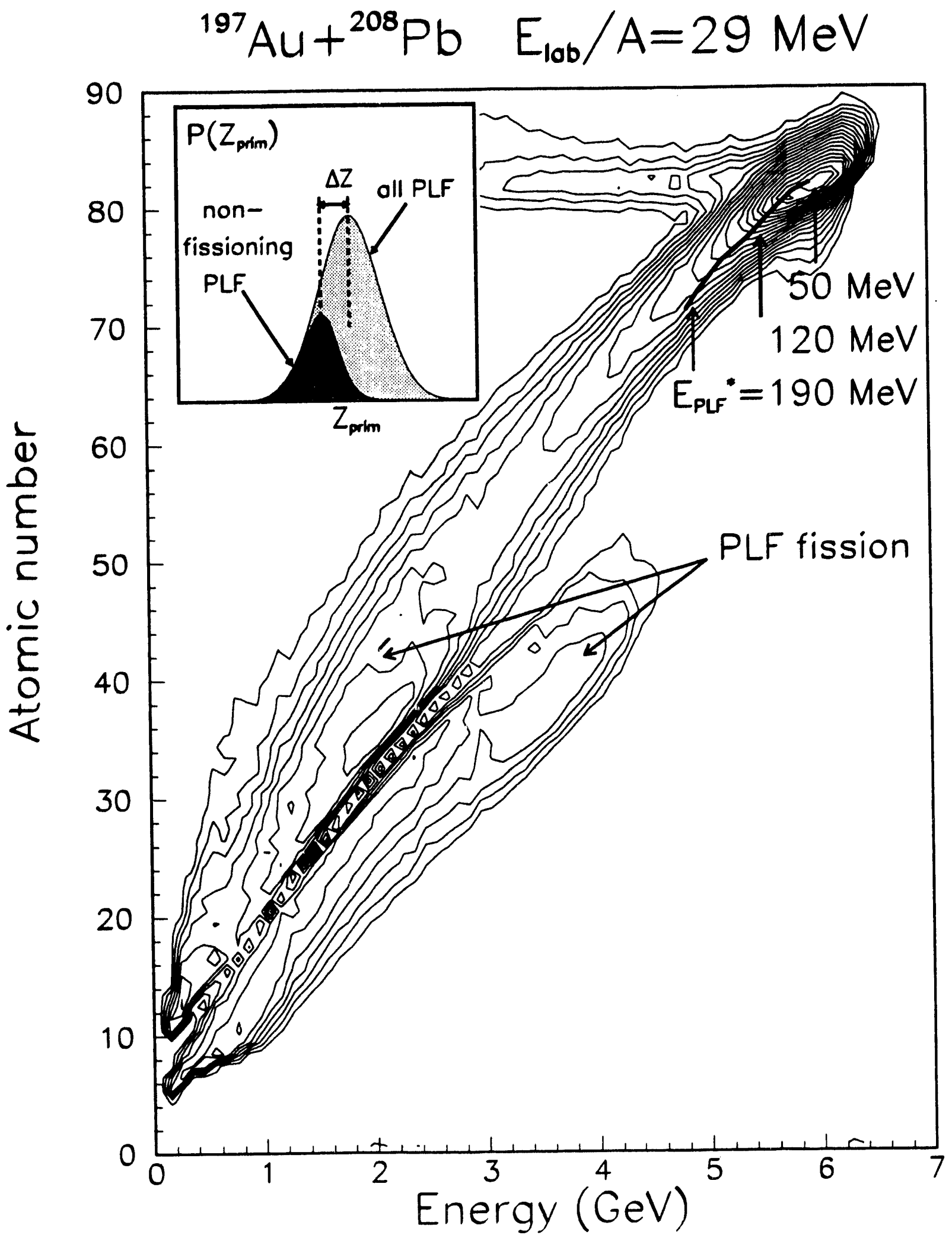

Figure IV.1 
${ }^{197} \mathrm{Au}+{ }^{208} \mathrm{~Pb} \quad E_{\text {lob }} / \mathrm{A}=29 \mathrm{MeV}$

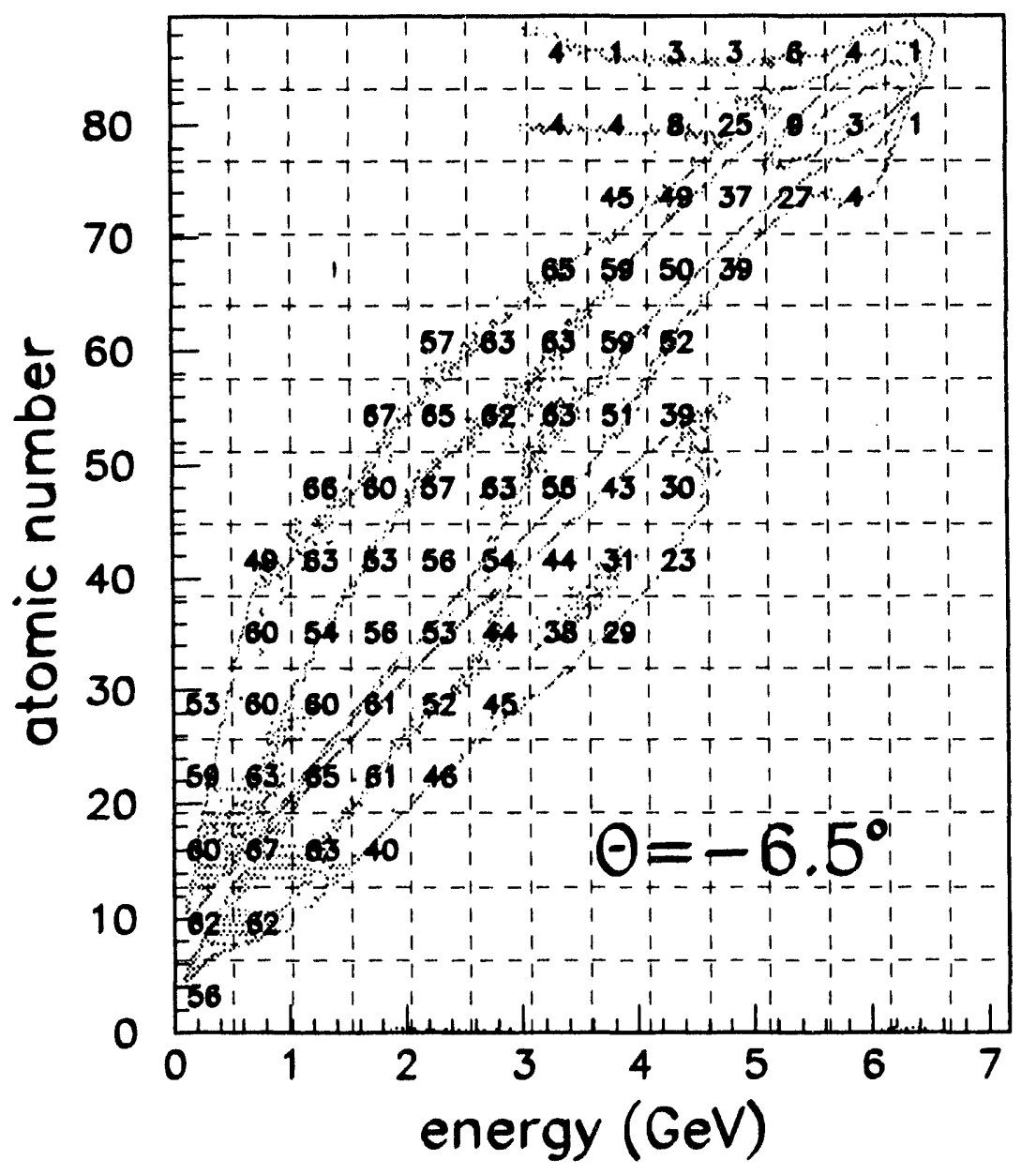

Figure IV.2 


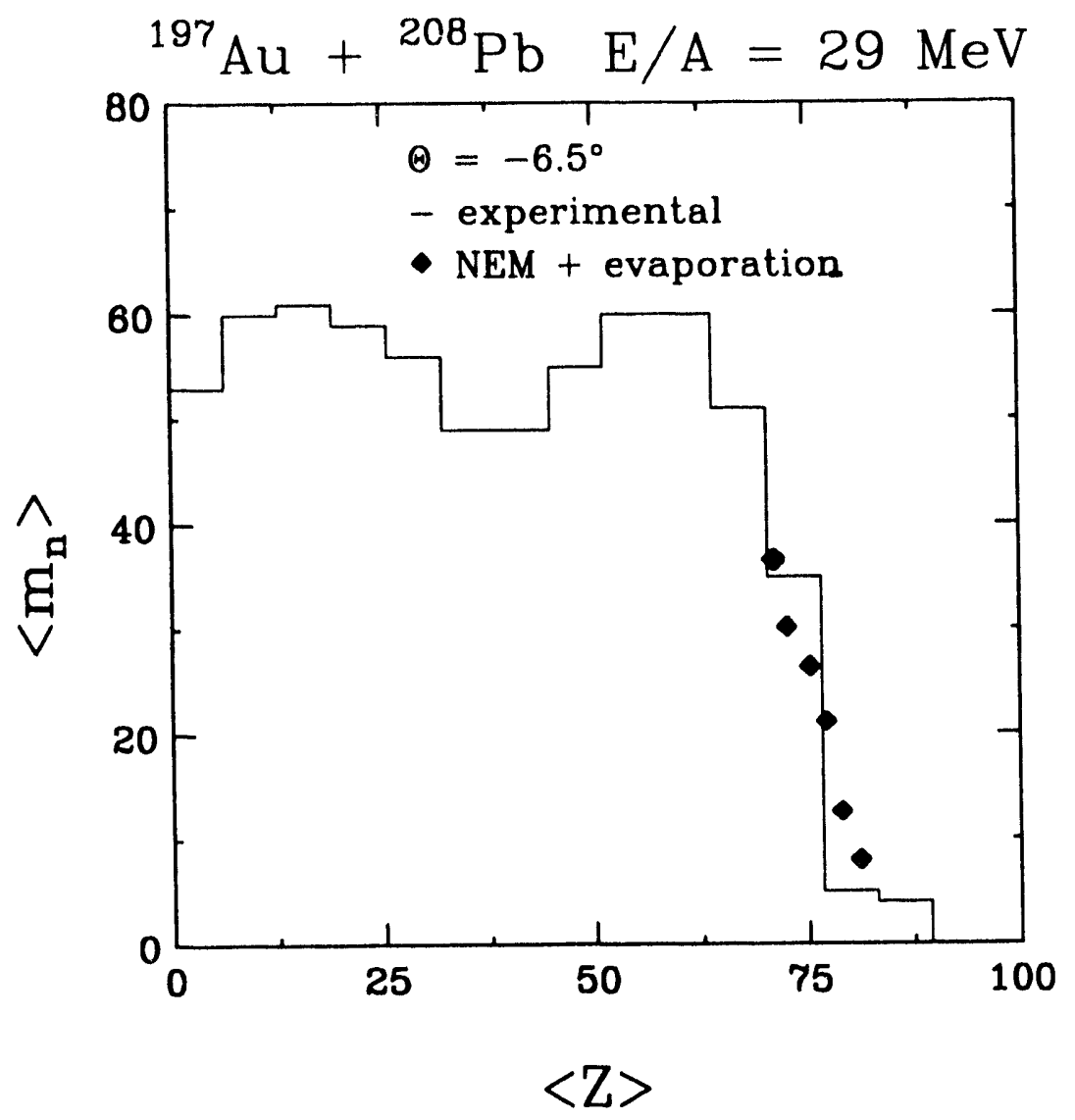

Figure IV.3 


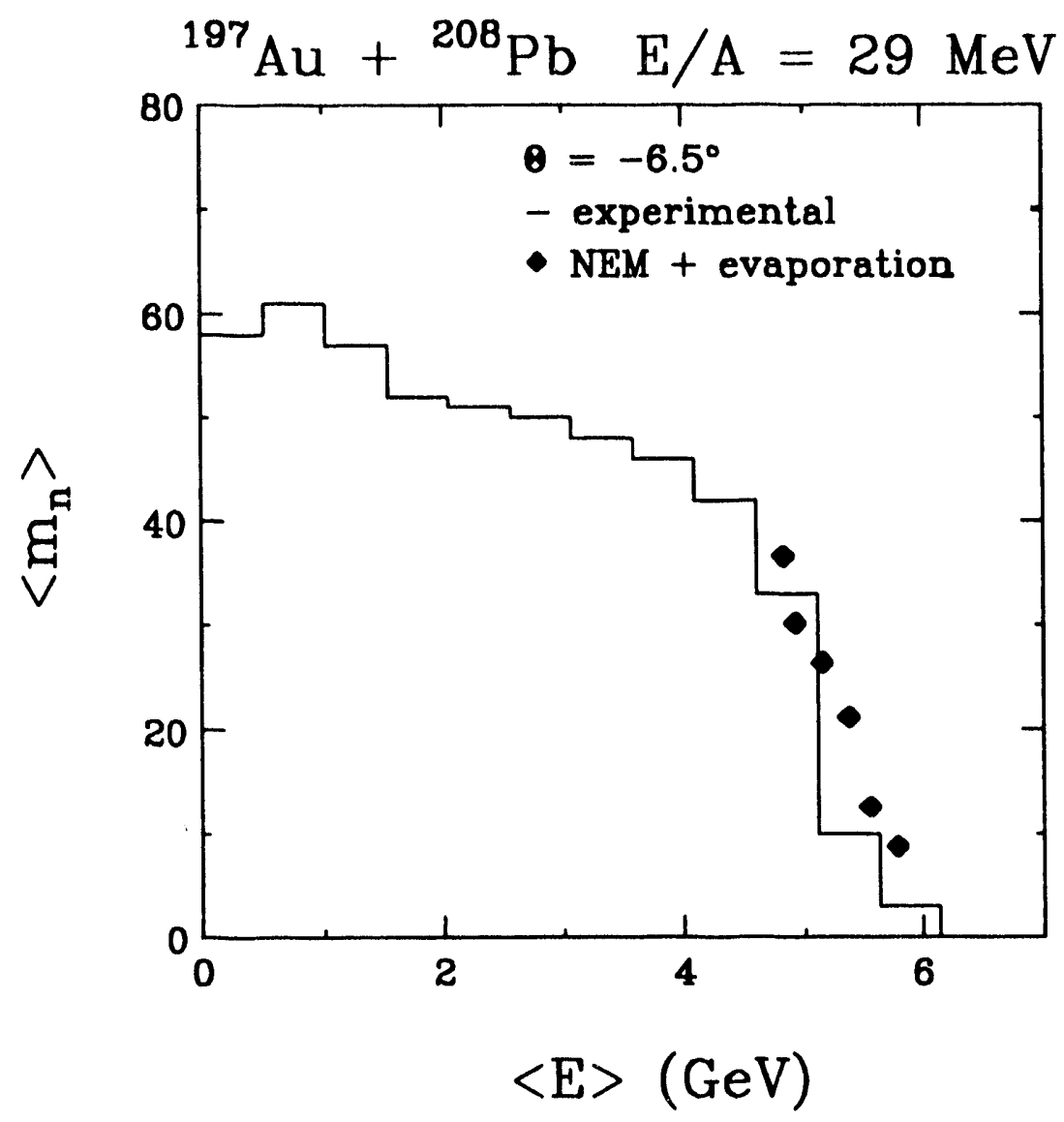




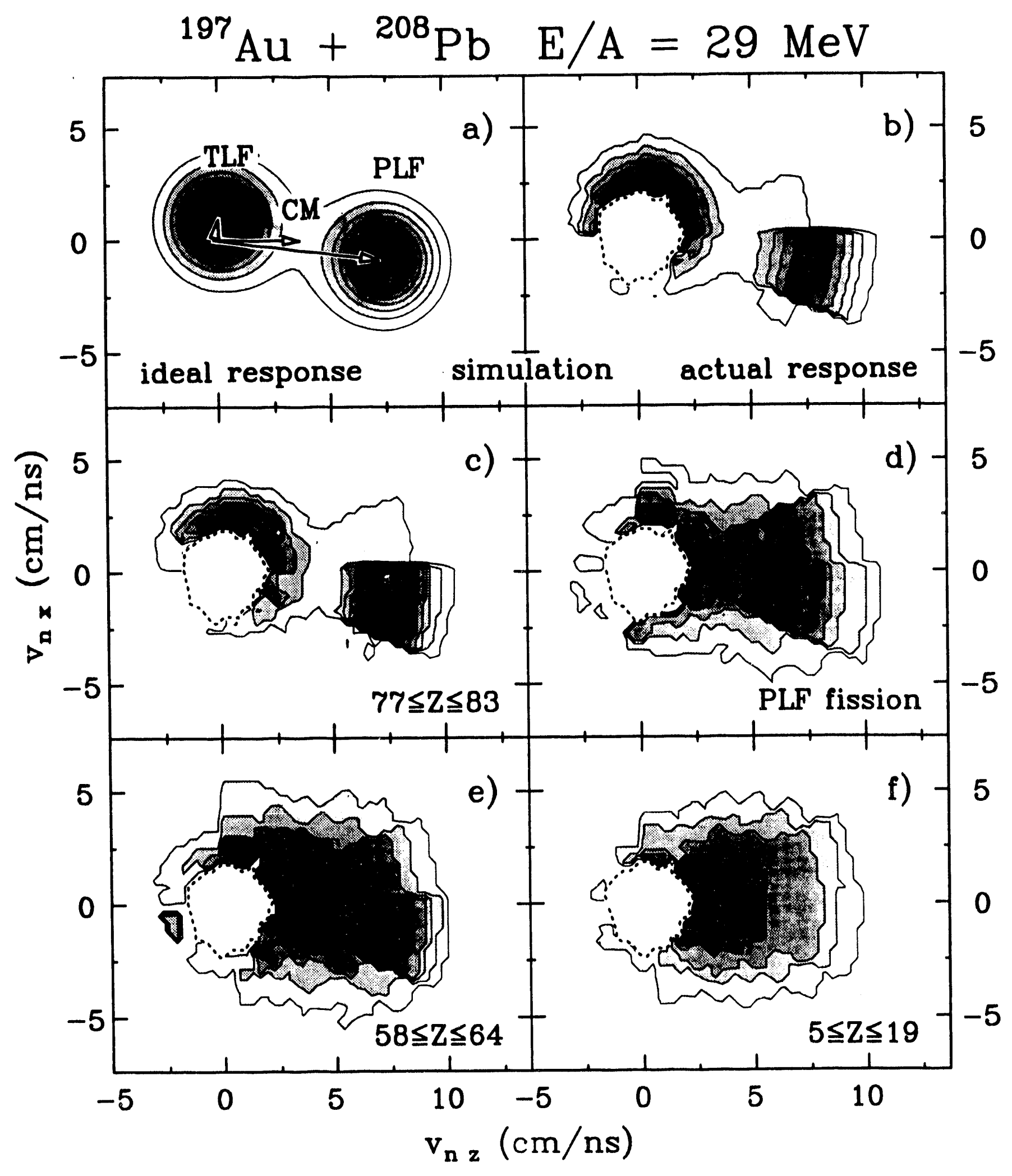

Figure IV.5 


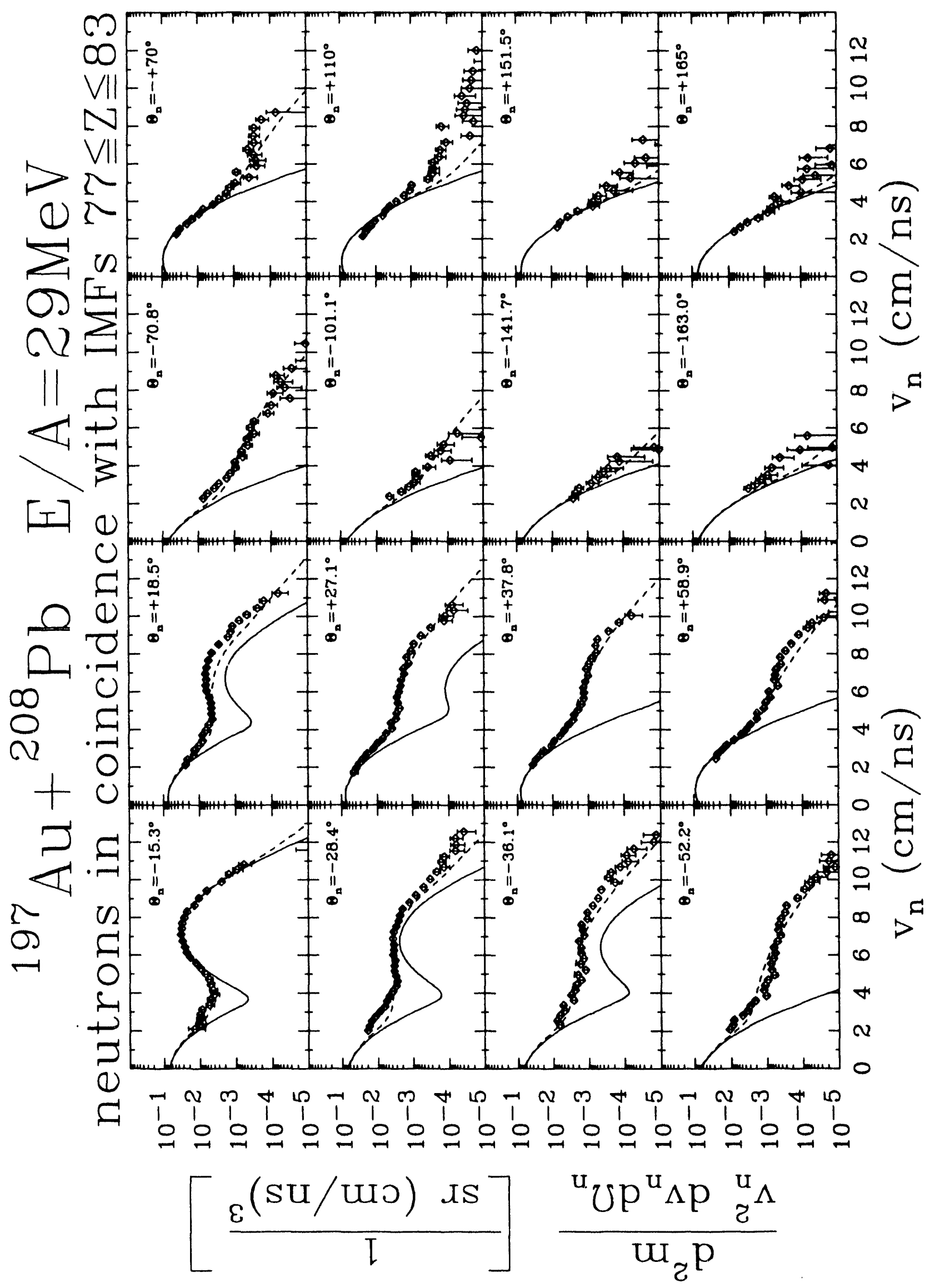

Figure IV.6 
$m_{\text {tot }}$

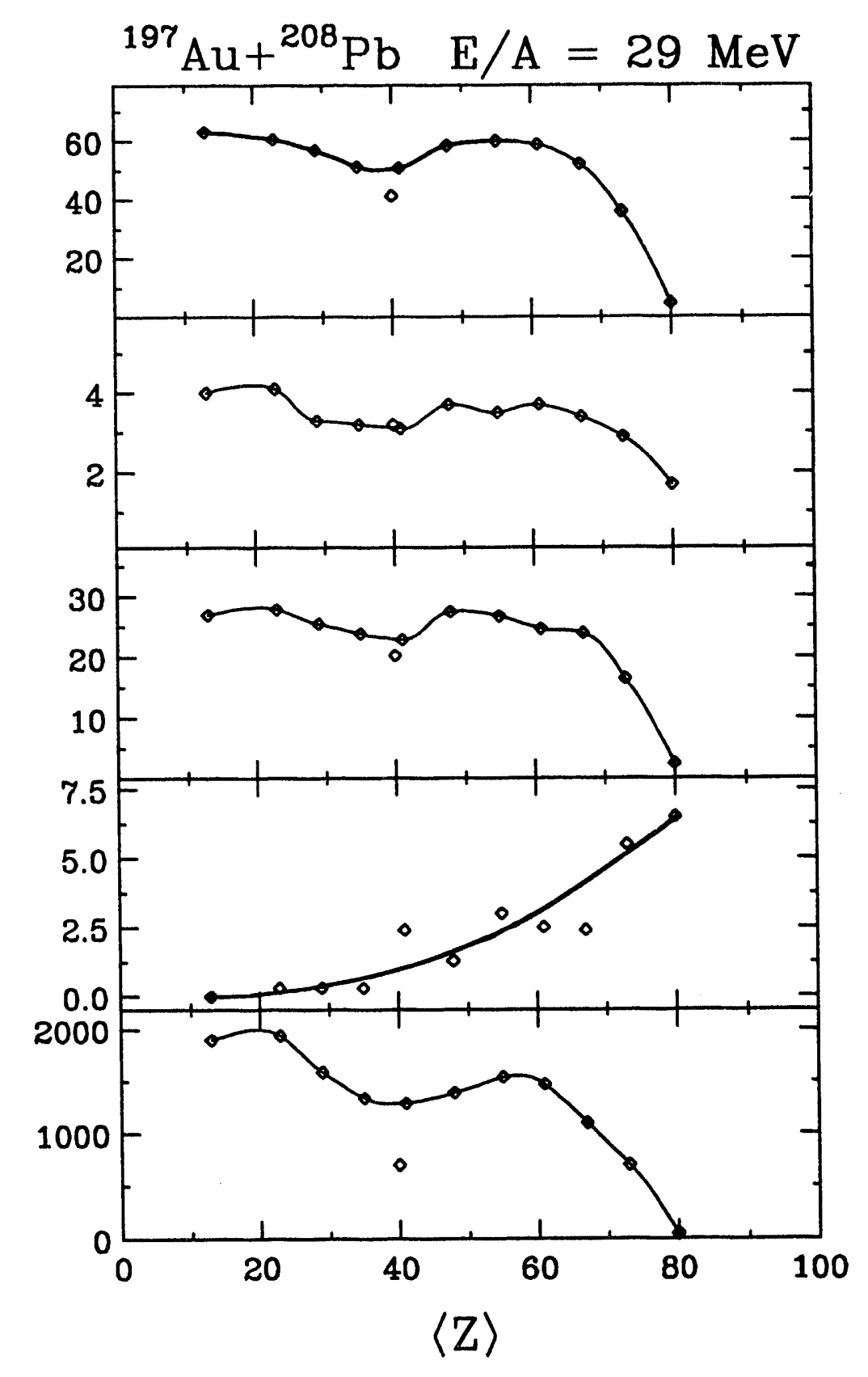

Figure IV.7 


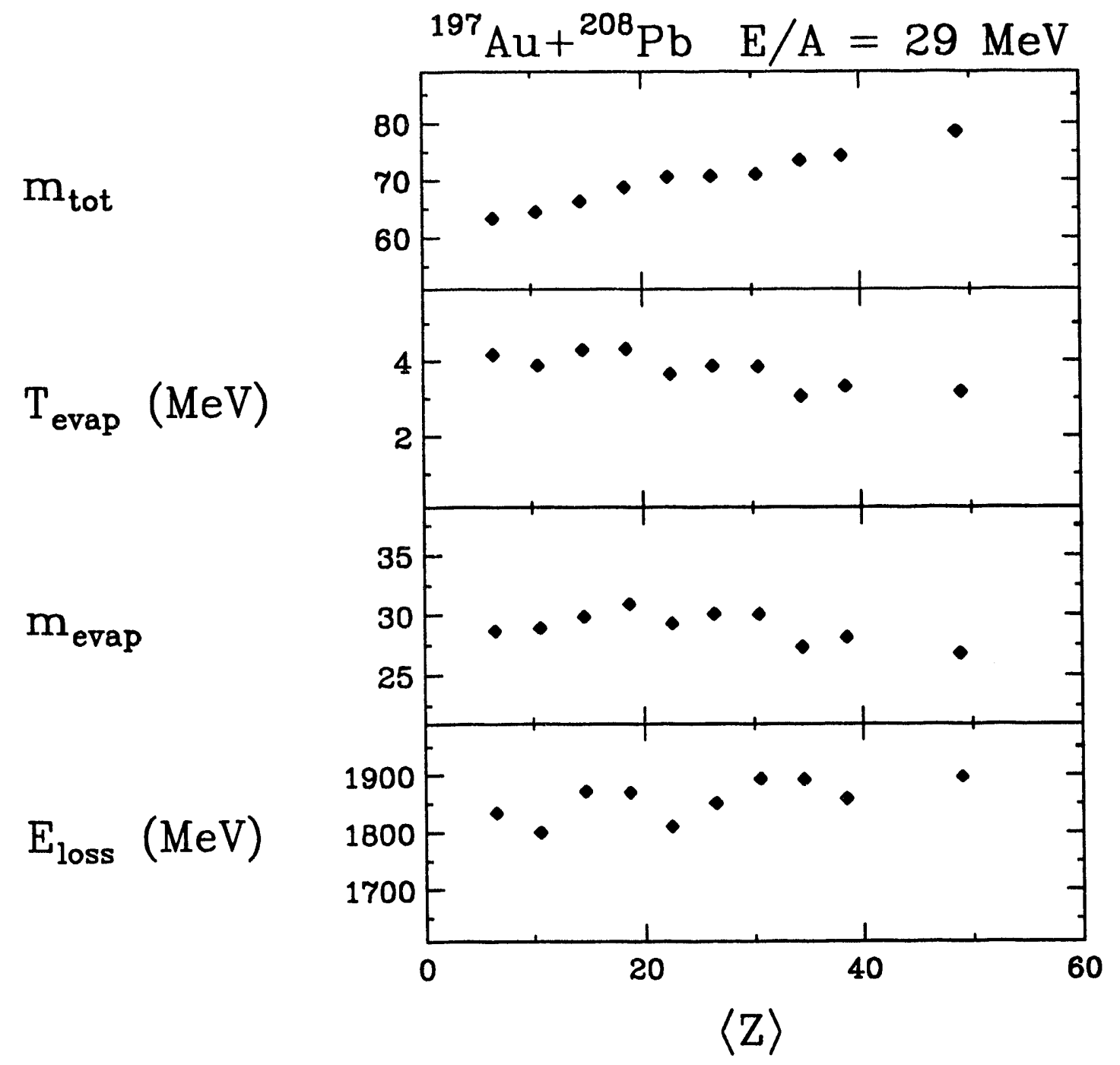

Figure IV.8 


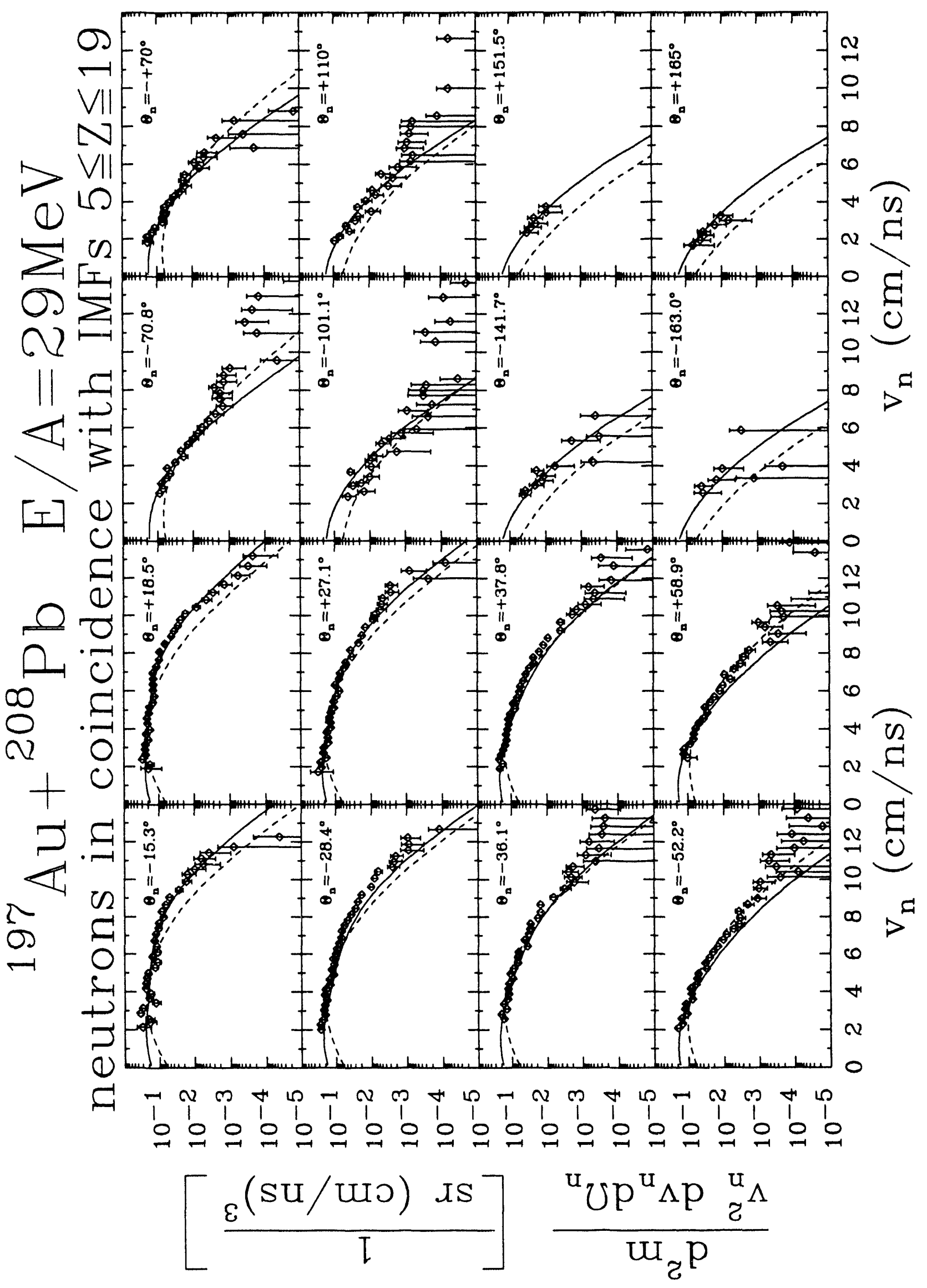

Figure IV.9 


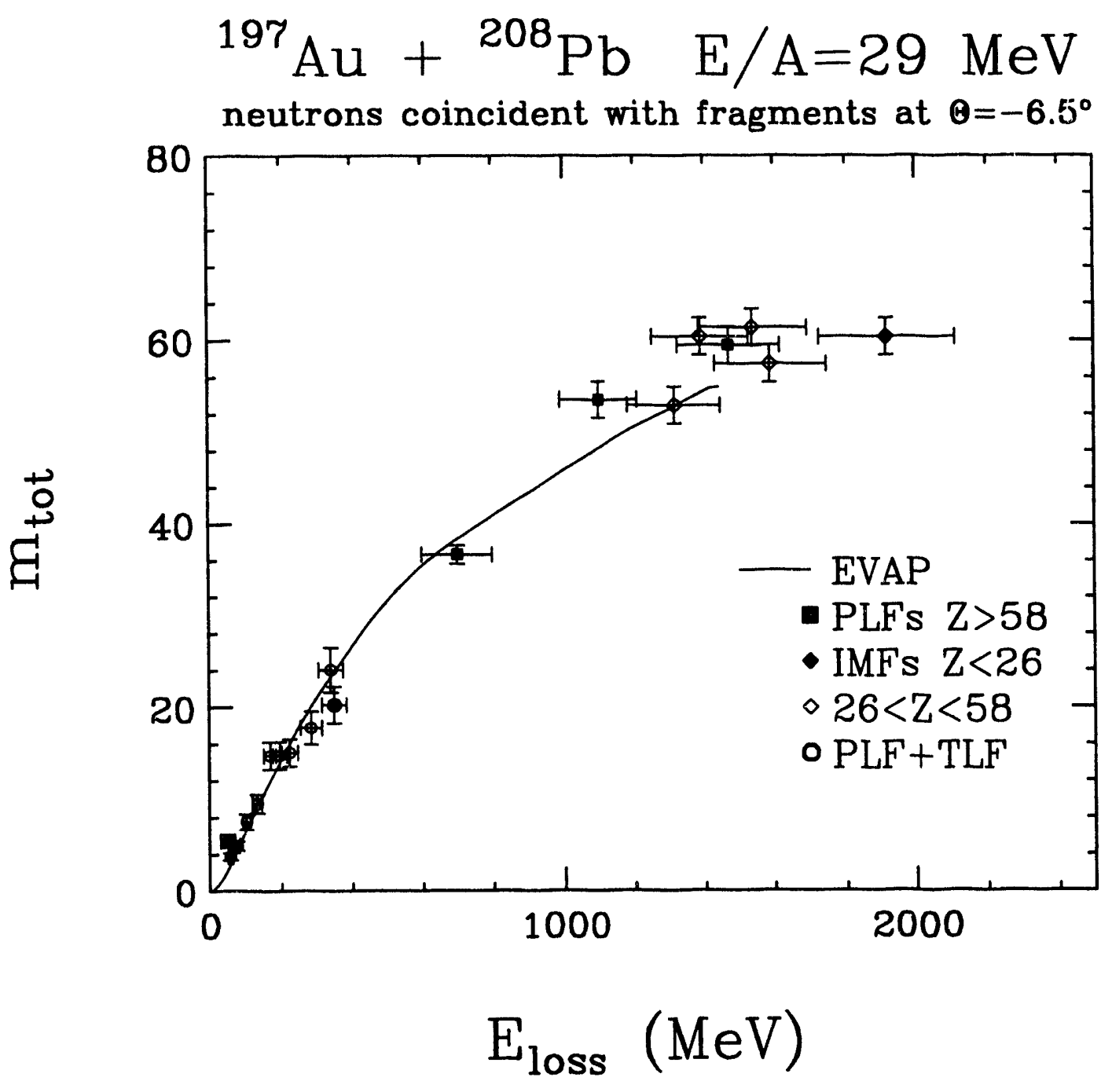

Figure IV.10 


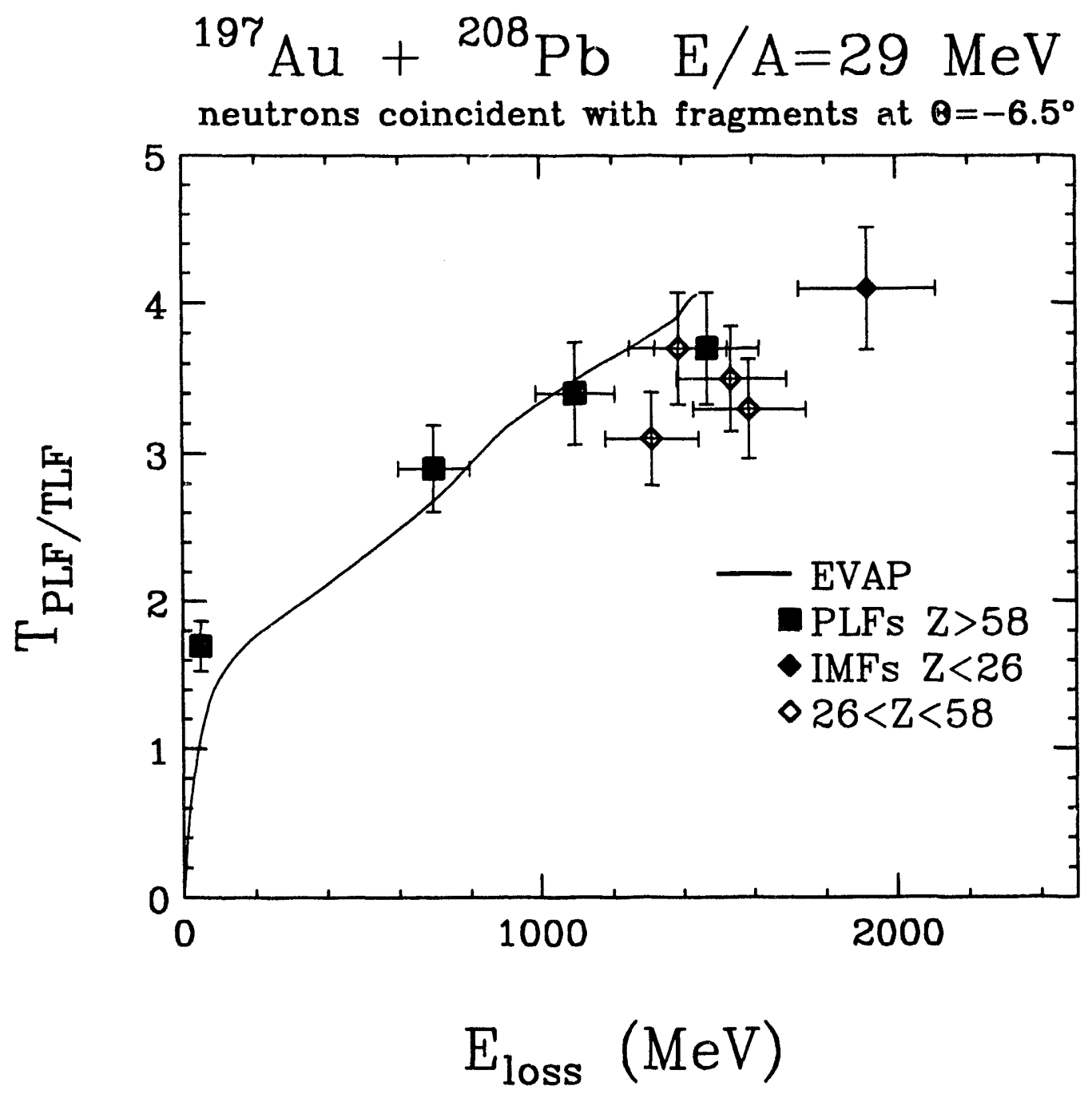




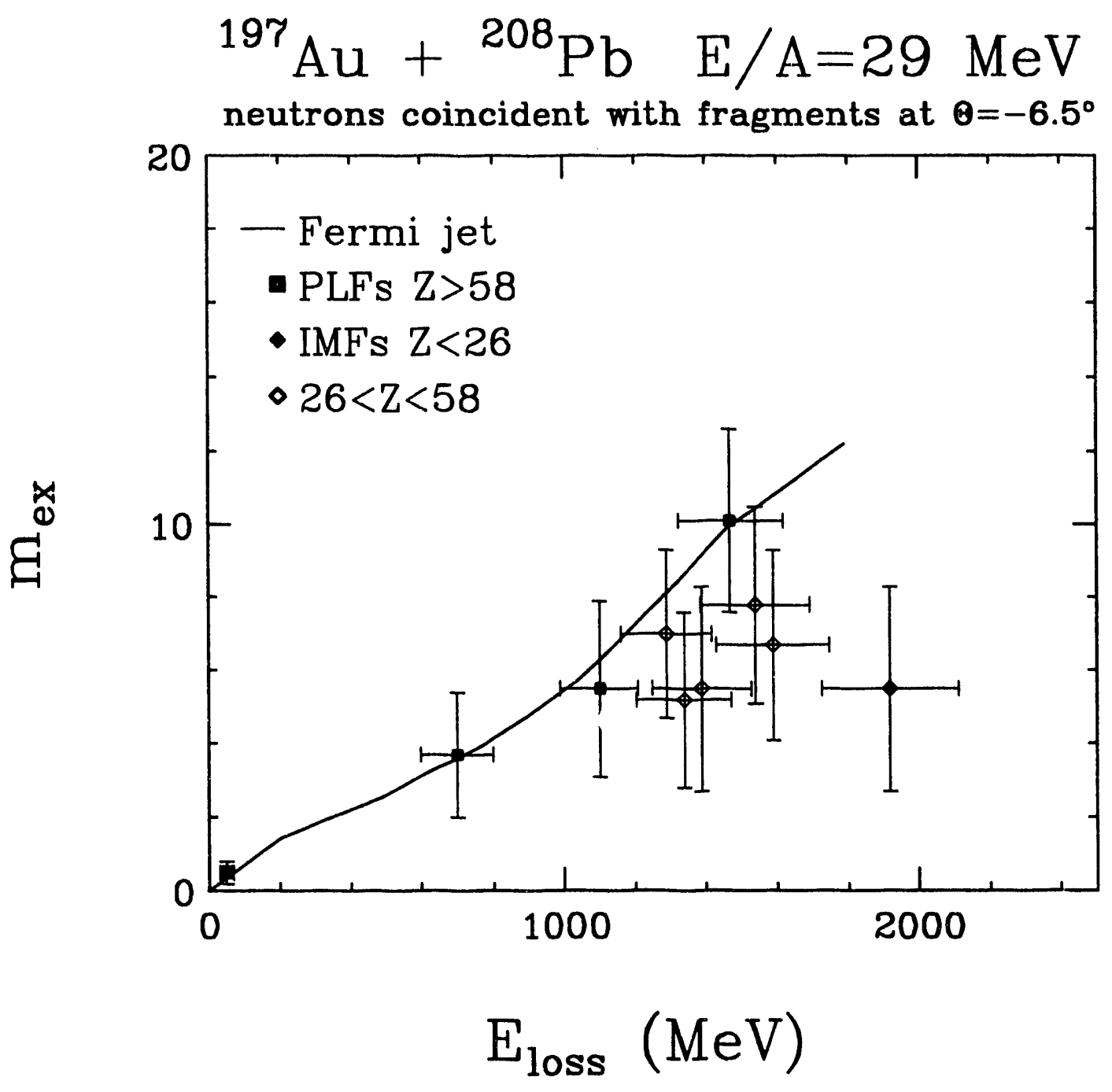




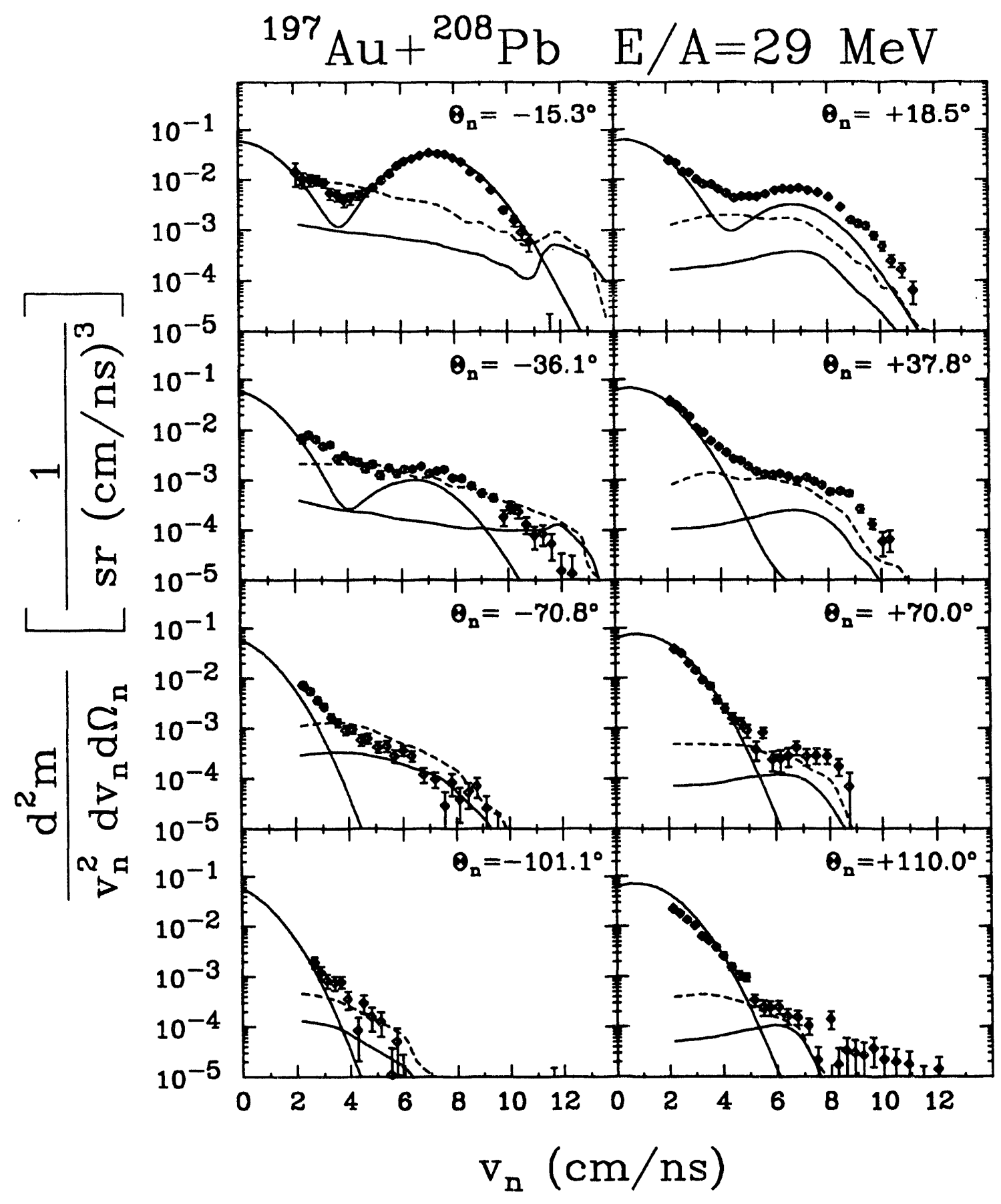

Figure IV.13 


\title{
OF PROJECTLE-LIKE FRAGMENT PRODUCTION IN THE REACTION ${ }^{200} \mathrm{Bi}+{ }^{136} \mathrm{Xe}$ AT $\mathrm{E}_{\mathrm{LAB}} / \mathrm{A}=28.2 \mathrm{MeV}$
}

\author{
S. P. BALDWIN, B. LOTT ${ }^{1}$, B. M. QUEDNAU ${ }^{2}$, \\ W. U. SCHRÖDER, B. M. SZABO, J. TŌKE \\ Nuclear Structure Research Laboratory and Department of Chemistry \\ University of Rochester, Rochester, NY 14627, USA \\ L. G. SOBOTKA, J. BARRETO ${ }^{3}$, R. J. CHARITY, \\ L. GALLAMORE, D. G. SARANTITES, D.W. STRACENER \\ Department of Chemistry \\ Washington University, St. Louis, MO 63130, USA \\ R. T. DE SOUZA 4 \\ National Superconducting Cyclotron Laboratory and Department of Physics \\ Michigan State University, East Lansing, MI 48824, USA
}

\begin{abstract}
Massive projectile-like fragments from the reaction ${ }^{209} \mathrm{Bi}+{ }^{136} \mathrm{Xe}$ at $\mathrm{E}_{\mathrm{lab}} / \mathrm{A}=$ 28.2 $\mathrm{MeV}$ were measured in coincides te with lighter charged products and neutrons, detected with a $4 \pi$ angular coverage. Correlations between the measured neutron multiplicity and massive-fragment energy, deflection angle, as well as the secondary and primary $\mathrm{Z}$ distributions, reveal features similar to those found for various systems at lower bombarding energies. Many of these correlations, in particular a dissipative orbiting feature, are characteristic of the damped reaction mechanism and are found consistent with the predictions of the nucleon exchange model.
\end{abstract}

\section{Introduction}

With the advent of accelerators providing beams of energetic, heavy projectiles, experimental studies of reactions between very heavy ions have recently become possible at intermediate bombarding energies. In reactions at bombarding energies of a few $\mathrm{MeV} /$ nucleon above the barrier, various observables associated with massive projectile-like and target-like fragments (PLF and TLF) were shown to be strongly

\footnotetext{
${ }^{1}$ On leave from CRN Strasbourg, France.

${ }^{2}$ Present address: GANIL,14021 Caen, France

${ }^{3}$ On leave from Instituto de Fisica da UFRJ, Rio de Janeiro, Brazil.

${ }^{4}$ Present address: Indiana University, Department of Chemistry, Bloomington, IN 47405, USA.
} 
correlated with the energy dissipated in a collision, a measure of impact parameter and interaction time. ${ }^{1}$ The observation of these correlations played an important role in developing an understanding of the dynamics of heavy-ion collisions in terms of a damped reaction mechanism and in formulating a quantitative reaction model, the one-body nucleon exchange model (NEM). ${ }^{2}$ A relatively clear picture of the damped reaction mechanism was obtained from studies of heavy systems such as $\mathrm{Bi}+\mathrm{Xe}$, for which other mechanisms, such as quasi-fission and fusion, were found to be absent.

For the system ${ }^{209} \mathrm{Bi}+{ }^{136} \mathrm{Xe}$, the above correlations were followed ${ }^{3,4}$ over a range of bombarding energies and were found to evolve smoothly with available kinetic energy, in agreement with the NEM. The question then arises to what extent, at bombarding energies per nucleon approaching the Fermi energy, heavy-ion collisions can still be explained within the same framework as at lower energies. To answer this question, the same ${ }^{209} \mathrm{Bi}+{ }^{136} \mathrm{Xe}$ reaction was studied ${ }^{5,6,7}$ at $\mathrm{E}_{\mathrm{lab}} / \mathrm{A}=28.2 \mathrm{MeV}$. As discussed below, in the experiment, massive fragments were measured in coincidence with both neutrons and charged products detected over nearly the entire solid angle.

\section{Experimental Technique}

The experiment was carried out at the National Superconducting Cyclotron Laboratory at Michigan State University. A beam of ${ }^{136} \mathrm{Xe}$ ions of $\mathrm{E}_{\mathrm{lab}} / \mathrm{A}=28.2 \mathrm{MeV}$ from the K1200 cyclotron bombarded a self-supporting $1.5 \mu \mathrm{g} / \mathrm{cm}^{2}{ }^{209} \mathrm{Bi}$ target placed in the center of a novel combination ${ }^{6}$ of particle detectors. This detector setup provided nearly full angular coverage for both neutrons and charged products. Neutrons were measured with the Rochester Neutron Multiplicity $\mathrm{Meter}^{8}$ (NMM) on an eventby-event basis. From the inherent design of the NMM, the highest sensitivity was achieved for neutrons from a slow-moving source, such as a TLF. Charged products were measured both with the St. Louis Dwarf Ball/Wall array ${ }^{9}$, configured with 95 phoswhich detectors, and with three multi-element silicon detector telescopes, which replaced one group of eight detectors of the Dwarf Wall. The Dwarf Ball/Wall measured light charged particles (LCP) with unit mass resolution, for particles with $\mathrm{Z} \leq$ 2 , and heavier charged products with unit $\mathrm{Z}$ resolution up to $\mathrm{Z} \approx 20$. The telescopes were placed at angles of $4.3^{\circ}, 13^{\circ}$, and $25^{\circ}$. The data on massive PLF discussed in this paper were obtained with the telescope $(64 \mu \mathrm{m} / 300 \mu \mathrm{m} / 3000 \mu \mathrm{m})$ placed forward of the grazing angle $\left(7.1^{\circ}\right)$ at $\left\langle\Theta_{\text {lab }}\right\rangle=4.3^{\circ}$. This telescope subtended an angular range of $2.6^{\circ}$ and provided two-dimensional position information.

\section{Dissipative Reactions}

\subsection{Reaction Cross Section}

The cross section for massive fragment $(Z \geq 35)$ production was determined by integrating the inclusive yield over $3.0^{\circ} \leq \Theta_{\text {lab }} \leq 5.6^{\circ}$. The absolute normalization was obtained $^{10}$ by assuming the Rutherford cross section for the elastic-scattering yield 
identified in the same detector. In order to distinguish reaction events from elastic scattering, a requirement of an associated neutron multiplicity $\mathrm{m}_{\mathrm{n}}>\mathbf{3}$ (or $\mathrm{E}_{\text {loss }}>\mathbf{5 0}$ $\mathrm{MeV}$ ) was imposed. The above integration over the measured angular distribution yielded for the cross section a value of $(2.3 \pm .5)$ b. A linear extrapolation of the angular distribution both to forward angles and backward angles, up to the grazing angle, gave an estimate of the total cross section for massive PLF production of (5.1 $\pm .5) \mathrm{b}$. This value represents a substantial fraction of the total reaction cross section of $5.9 \mathrm{~b}$ predicted by systematics. ${ }^{11}$

\subsection{Massive-Fragment $Z$ and Energy Distributions}

Figure 1 shows a two-dimensional distribution of the fragment yield measured at $\left\langle\Theta_{\text {lab }}\right\rangle=4.3^{\circ}$, plotted versus atomic number $(\mathrm{Z})$ and energy $\left(\mathrm{E}_{\mathrm{lab}}\right)$. A well-defined ridge of yield is seen to extend from the elastic-scattering peak at $\mathrm{Z}=54$ and $\mathrm{E}_{\text {lab }} \approx$ $3.8 \mathrm{GeV}$. For $\mathrm{Z} \geq 35$, the intensity along the ridge line first decreases with fragment $\mathrm{Z}$ and $\mathrm{E}_{\mathrm{lab}}$, while for $\mathrm{Z}<35$, it remains relatively constant down to the lightest fragments measured. The almost linear character of the correlation between $\mathrm{Z}$ and $\mathrm{E}_{\text {lab }}$ observed in Figure 1, for $\mathrm{Z}<40$ suggests that fragments have similar velocities. No clear evidence for sequential fission of the primary excited PLF is seen in Figure 1.

In the past, similar distributions for other systems ${ }^{12,13}$ at intermediate bombarding energies were discussed in terms of participant-spectator ${ }^{14,13}$ or incomplete fusion ${ }^{15}$ models. Such models predict cold massive PLF to be deflected to forward angles, with velocities close to the beam velocity. For orientation, straight lines are shown in Figure 1 indicating three different values of constant velocity or $\mathrm{E} / \mathrm{A}$. Obviously, the average PLF energy per nucleon for $\mathrm{Z}<40$ is approximately $19 \mathrm{MeV}$ (dashed line), which is significantly lower than the beam energy of $28.2 \mathrm{MeV} / \mathrm{A}$ (solid line). Very few events are observed with fission-like energies that could be associated with full damping of the total kinetic energy (dot-dashed line).

It was also found in the present work that the associated neutron multiplicity increases with decreasing average velocity of PLF, confirming earlier results ${ }^{1,16,17}$ according to which both these observables can serve as measures of the energy dissipated in a reaction.

\subsection{Dissipative Orbiting}

Observation of characteristic correlations between the amount of dissipated kinetic energy and the deflection angle of the PLF has played an important role in studying the dyuamics of damped reactions at lower bombarding energies. Such correlations, visualized in a two-dimensional (Wilczyński) plot $^{1}$ of the yield versus the deflection angle and the kinetic energy of the PLF, have revealed the evolutionary character of the underlying processes. The observation of such correlations has led to the introduction of the notion of friction forces to heavy-ion physics and to the development of various models of dissipative interaction dynamics.

Figure 2 shows the observed PLF yield, plotted versus the PLF deflection angle $\Theta_{\text {lab }}$ and the associated neutron multiplicity. Since the neutron multiplicity is 
a good measure of the dissipated energy, this figure may be regarded as an analog of the Wilczyniski plot. As seen in this figure, the degree of energy dissipation is strongly correlated with the PLF deflection angle, indicative of a dissipative orbiting process. Clearly, an increasing amount of kinetic energy is converted into heat, as the interacting dinuclear complex rotates toward the smaller angles. The solid line in Figure 2 represents the correlation ridge line predicted by model simulations incorporating classical trajectory calculations based on the NEM, as well as simplified neutron evaporation calculations for the sequential decay of the primary PLF and TLF. In these calculations, the "modified" proximity potential" was assumed for the nuclear interaction. As seen in Figure 2, such model calculations account quite well for the main ridge of the observed yield, however, they predict a continuation of this ridge into the range of negative scattering angles, which is not observed in the data.

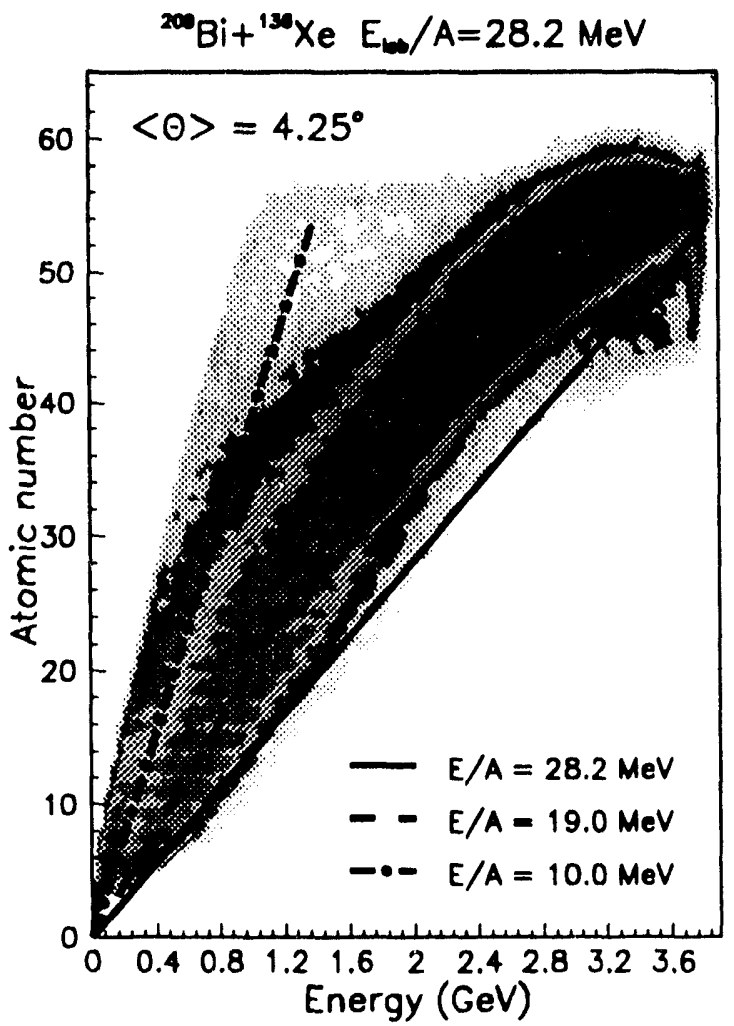

Figure 1. Yield of fragments detected in the telescope at $4.3^{\circ}$ vs. atomic number $Z$ and kinetic energy $E$. The solid, dashed, and dotdashed lines correspond to constant energy per nucleon of $28.2,19$, and $10 \mathrm{MeV}$, respectively.

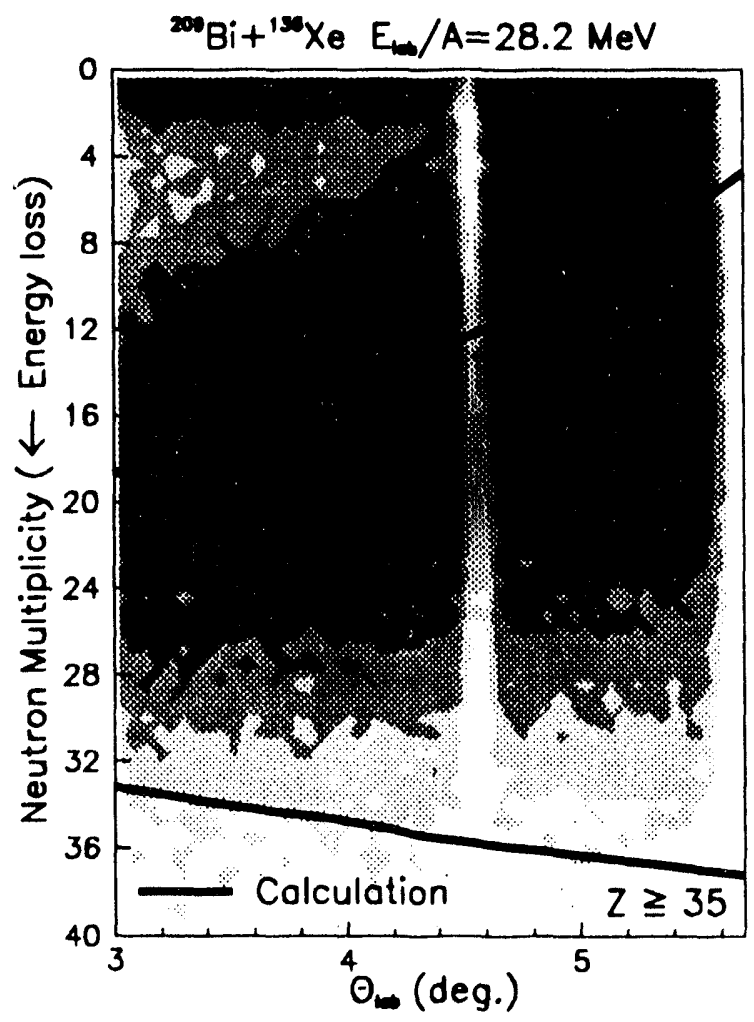

Figure 2. Observed PLF yield, plotted versus the PLF deflection angle $\Theta_{\text {lab }}$ and the associated neutron multiplicity. The line represents the correlation ridge line predicted by model simulations.

\subsection{Binary Collision Dynamics}

Important features of the reaction dynamics are expected to be reflected in the primary-product $\mathrm{Z}$ distributions. For example, for a damped reaction mechanism, 
such distributions provide a rather direct measure of the strength and direction of the driving forces governing nucleon exchange in the system. As in the present experiment light charged particles were measured in coincidence with PLF, such primary PLF $\mathrm{Z}$ distributions can be reconstructed, provided the light charged particles with $\mathrm{Z} \leq$ 3 (LCP) evaporated from these fragments are identified. For the purpose of such identification, measured LCP emission patterns were analyzed.

Figure 3 shows Galilei-invariant velocity distributions $d^{2} \sigma / v_{\perp} d v_{\|} d v_{\perp}$ of protons in the PLF reference frame, for different bins in atomic number $Z_{P L F}$ of the coincident PLF. In this figure, $v_{\|}$and $v_{\perp}$ denote the velocity components parallel and perpendicular, respectively, to the relative velocity of PLF and TLF. For all bins in $Z_{P L F}$, the velocity distributions are seen to exhibit bimodal patterns, with two semi-circular ridges corresponding to LCP emission from excited PLF and TLF. It has already been shown elsewhere ${ }^{6,7}$ that LCP exhibit similar emission patterns, for a much broader class of reactions than studied here. The average LCP multiplicities associated with PLF and TLF were determined from the yields in the respective invariant velocity distributions, corrected for non-unit detection efficiencies. The LCP multiplicities for the two fragments were found to be approximately equal to each other.

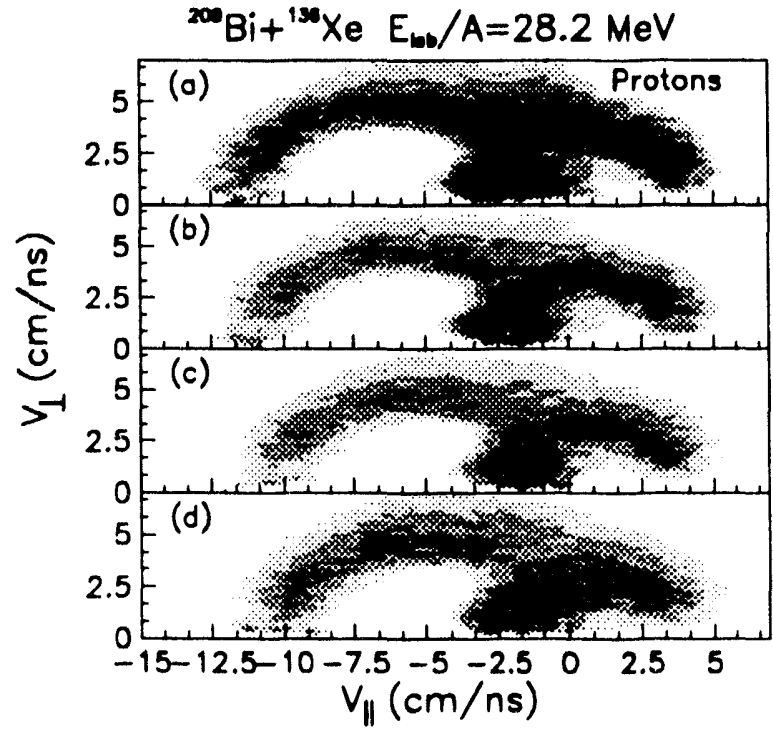

Figure 3. Galilei-invariant velocity distributions of protons as a function of $v_{\|}$and $v_{\perp}$ in the PLF reference frame, for bins in $Z$-value of the PLF, $51 \leq Z \leq 60,41 \leq Z \leq 50,31 \leq Z \leq 40$, and $21 \leq Z \leq 30$ for panels $a, b, c$, and $d$, respectively.

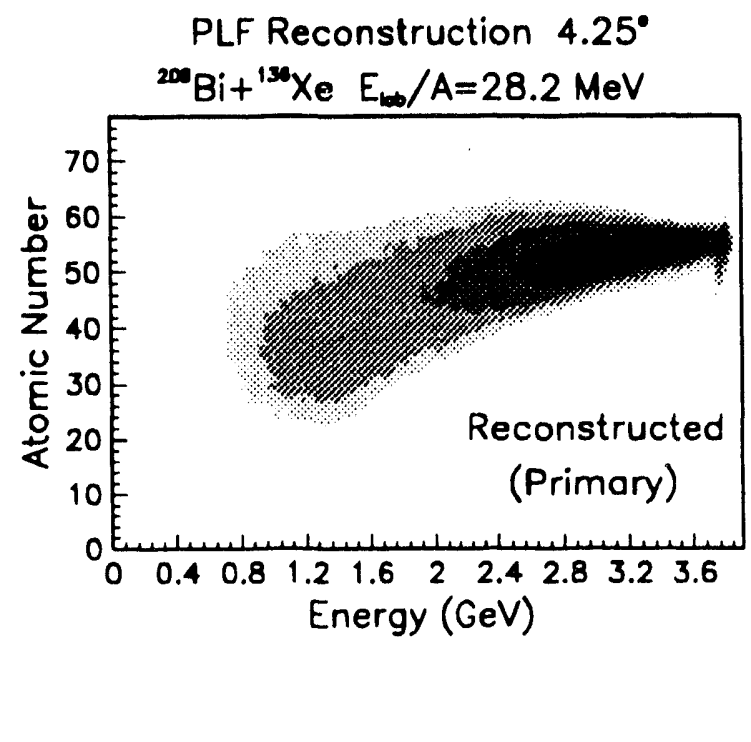

Figure 4. PLF yield plotted versus $E_{\text {lab }}$ and reconstructed primary $\mathrm{Z}$.

\subsection{Primary Z Distributions}

The primary PLF atomic number was calculated event by event by adding to the atomic number of the identified secondary PLF the total charge of LCP attributed to that PLF, corrected for the LCP detection efficiency. Figure 4 shows the PLF yield 
plotted versus reconstructed primary $Z$ and $E_{\text {lab }}$ of these fragments. The primary atomic number distribution is seen to drift only weakly with decreasing PLF energy, while undergoing a relatively substantial symmetric broadening. More detailed representations of the primary $Z$ distributions are shown in Figures 5 and 6.

Figure 5 shows the evolution of the PLF primary $\mathrm{Z}$ distributions with increasing neutron multiplicity. The distributions in this figure have almost Gaussian shapes, broadening with increasing energy dissipation, in a fashion similar to what is observed at lower bombarding energies. Values of centroids and variances of these $\mathrm{Z}$ distributions are plotted in Figure 6 as functions of the neutron multiplicity and are compared to the results of NEM calculations. Considering the uncertainties introduced by the analysis procedure, a reasonably quantitative agreement is observed between the data and the predictions of the NEM, for both moments.

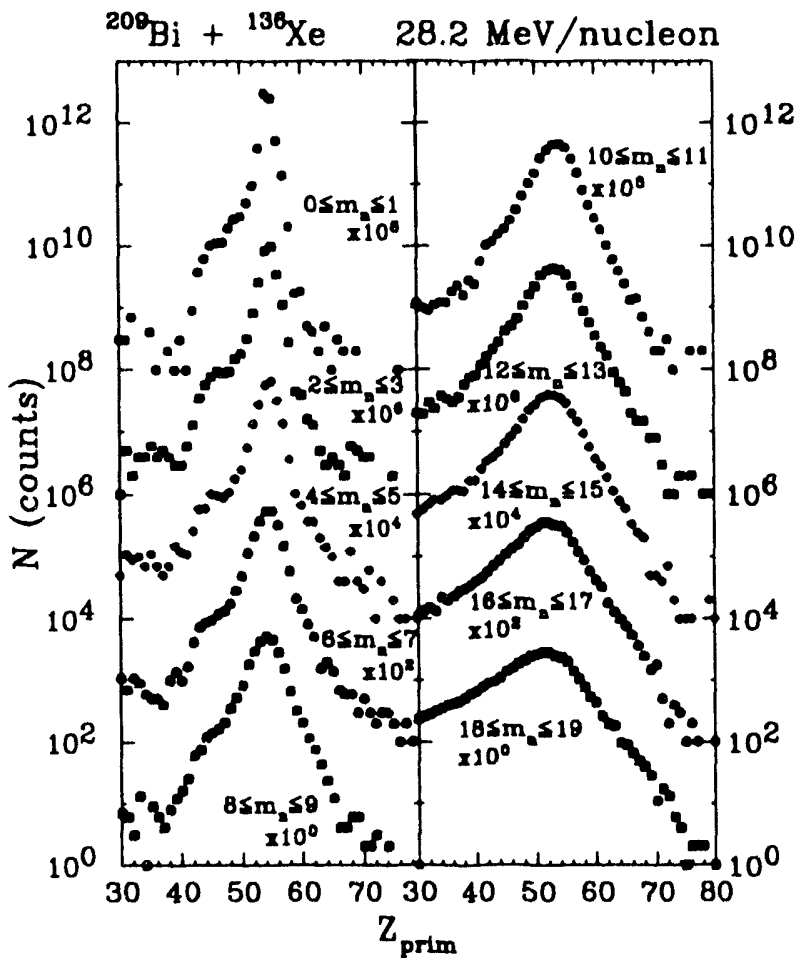

Figure 5. Distributions of reconstructed primary atomic numbers $\mathrm{Z}_{\text {prim }}$, for fragments detected at $\left\langle\Theta_{\text {lab }}\right\rangle=4.3^{\circ}$, for various bins in $\mathbf{m}_{\mathbf{n}}$.
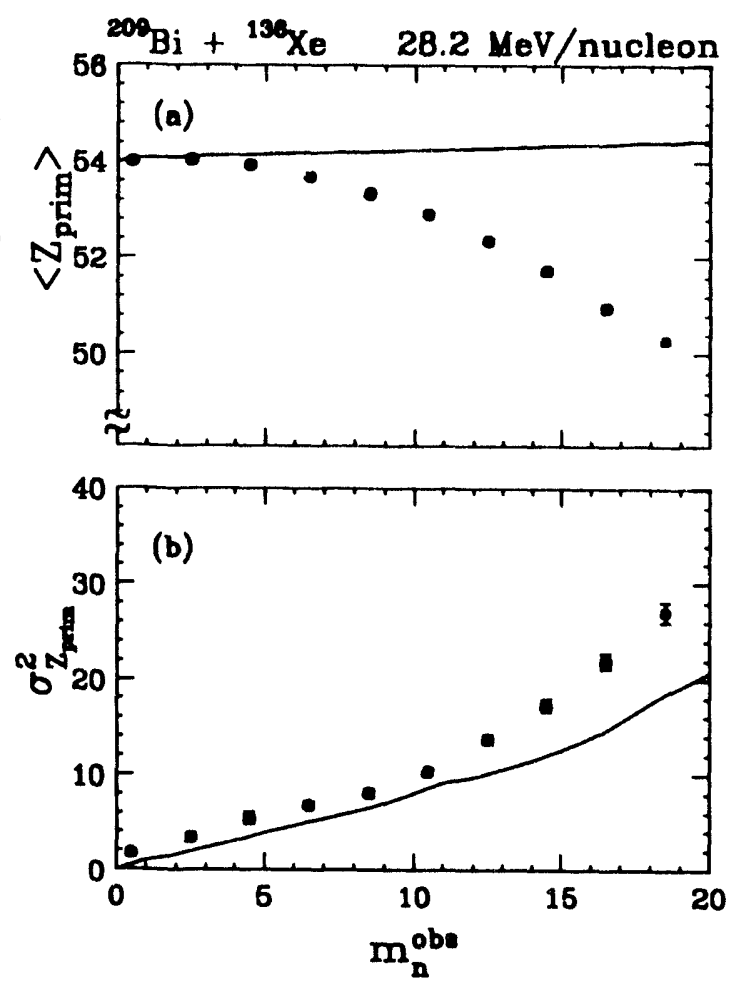

Figure 6. Experimental centroids (a) and variances (b) of the distributions in Figure 5 (solid symbols) and results of NEM calculations (solid lines) plotted as function of $m_{n}$.

\section{Conclusions}

The present study of massive, projectile-like fragments from the reaction ${ }^{209} \mathrm{Bi}+$ ${ }^{136} \mathrm{Xe}$ at $\mathrm{E}_{\mathrm{lab}} / \mathrm{A}=28.2 \mathrm{MeV}$ reveals several features reminiscent of damped reactions at lower bombarding energies. The cross section associated with such massive fragment production is estimated at $(5.1 \pm .5) \mathrm{b}$, accounting for most of the $5.9 \mathrm{~b}$ of total 
reaction cross section predicted by systematics and indicating that a representative event sample was explored in the present work. A strong dependence of the PLF atomic number and deflection angle on neutron multiplicity or dissipated energy is observed. The massive fragments are seen to be produced in binary, orbiting-type collisions, compatible with the scenario underlying the nucleon exchange model.

\section{Acknowledgements}

This work was supported by the U.S. Department of Energy under grant No. DEFG02-88ER40414 (Univ. of Roch.), DE-FG02-87ER403160 and DE-FG02-88ER40406 (Wash. Univ.), and by the National Science Foundation under grant No. PHY-8913815 (NSCL).

\section{References}

[1] W.U. Schröder and J.R. Huizenga, in Treatise on Heavy-Ion Science, ed. D.A. Bromley (Plenum, New York, 1984) Vol. 2, p. 113, and ref. therein.

[2] J. Randrup, Nucl. Phys. A307, 319 (1978); A327, 490 (1979); A38s, 468 (1982). J. Døssing et al., Nucl. Phys. A433, 215 (1985); A433, 280 (1985).

[3] W.W. Wilcke et al., Phys. Rev. C 22, 128 (1980); W.U. Schröder et al., Phys. Rev. Let. 36, 514 (1976), Phys. Rep. 45C, 301 (1978); H.J. Wollersheim et al., Phys. Rev. C 24, 2114 (1981).

[4] J.R. Huizenga et al., Phys. Rev. Let. 37, 885 (1976); W.U. Schröder et al., Phys. Let. 71B, 283 (1977), Phys. Rev. Let. 44, 308 (1980); H.J. Wollersheim et al., Phys. Rev. C 25, 338 (1982).

[5] W.U. Schröder, Nucl. Phys. A538, 439c (1992).

[6] B. Lott et al., in Advances in Nuclear Dynamics-Proc. of the Workshop on Nuclear Dynamics VIII, Jackson Hole, WY, USA, Jan. 18-25, 1992, eds. W. Bauer and B. Back (World Scientific, Singapore, 1992), p. 62.

[7] B. Lott et al., Phys. Rev. Let. 68, 3141 (1992).

[8] S.P. Baldwin et al., USDOE Progress Report 1990, DOE/ER/40414-3, p. 247.

[9] D.W. Stracener et al., Nucl. Instr. and Meth. in Phys. Res. A294, 485 (1990).

[10] J.R. Birkelund et al., Phys. Rev. C 13, 133 (1976).

[11] W.W. Wilcke et al., Atomic Data and Nuclear Data Tables 25, 389 (1980).

[12] R. Lucas et al., Nucl. Phys. A464, 172 (1987).

[13] O. Granier et al., Nucl. Phys. A481, 109 (1988).

[14] A.S. Goldhaber, Phys. Let. 53B, 306 (1974),

[15] D. Guerreau, in Nuclear Matter and Heavy-Ion Collisions, eds. M. Soyeur, H. Flocard, B. Tamain, and M. Porneuf (Plenum, New York, 1989) and ref. therein.

[16] D. Pade et al., Phys. Rev. C 43, 1288 (1991).

[17] M. Morjean et al., Nucl. Phys. A524, 179 (1991). 
Dynamical production of intermediate-mass fragments in peripheral ${ }^{209} \mathrm{Bi}+{ }^{136} \mathrm{Xe}$ collisions at $E_{\text {lab }} / \mathrm{A}=28.2 \mathrm{MeV}$

B. Lott, ${ }^{*}$ S. P. Baldwin, B. M. Szabo, B. M. Quednau, ${ }^{\dagger}$ W. U. Schröder, and J. Töke Department of Chemistry and Nuclear Structure Research Laboratory, University of Rochester, Rochester, New York 14627

L. G. Sobotka, J. Barreto ${ }^{\ddagger}$ R. J. Charity, L Gallamore, D. G. Sarantites, and D. W. Stracener Department of Chemistry, Washington University, St. Louis, Missouri 63130

\author{
R. T. de Souza \\ Department of Physics and National Superconducting Cyclotron Laboratory, \\ Michigan State University, East Lansing, Michigan 48824
}

\begin{abstract}
Intermediate-mass fragments from the reaction ${ }^{209} \mathrm{Bi}+{ }^{136} \mathrm{Xe}$ at $\mathrm{E}_{l a b} / \mathrm{A}=28.2 \mathrm{MeV}$ have been measured in coincidence with other charged reaction products, as well as with neutrons, using a complex $4 \pi$ detector setup. For peripheral collisions, multiplicities and emission patterns are observed for intermediate-mass fragments that can largely not be accounted for by evaporation from equilibrated nuclear systems. These patterns are shown to be indicative of a dynamical production mechanism, possibly of a multiple neck rupture.
\end{abstract}

PACS number: $25.70 . \mathrm{Lm}$ 
In recent years, studies of heavy-ion reactions at bombarding energies per nucleon comparable to nucleonic Fermi energie have focused considerable interest on the apparent overproduction of intermediate-mass fragments (IMF) ${ }^{1,2}$. The fact that these large observed IMF multiplicities cannot be reconciled with standard statistical-model predictions for the decay of hot, but otherwise "normal" nuclear systems has prompted modeling of various scenarios favoring copious production of IMF's,4. As the highest IMF multiplicities were found to be associated with large amounts of heat generated in rather central collisions, the production scenarios suggested involve often a liquid-gas phase transition in hot nuclear matter, induced perhaps by a compression-decompression cycle of the nuclear system. While many important features of IMF production find a qualitative or even quantitative explanation within the framework of such proposed new models, experimental evidence has mounted at the same time $e^{5,6}$ for a dynamical rather than a statistical nature of at least some of the IMF production processes. So iar, the presence of such dynamical processes has been largely overlooked. These dynamical processes have, however, a significant potential of carrying unique information, e.g., on the dynamics of the fast approach and possibly on the reseparation stage of the constituent fragments of the system and, hence, deserve a thorough study.

The present paper focuses on the dynamical aspects of IMF production in intermediateenergy heavy-ion reactions. The analysis described below is limited to rather peripheral collisions, selected using different available experimental criteria. In such collisions, the presence of dynamical IMF production mechanisms is most clearly seen and was actually discovered in the present work. Furthermore, such peripheral collisions are associated with only moderate excitation energies and follow mostly well-defined binary reaction scenarios ${ }^{7}$, both features facilitating analysis. The excitation energy range involved is largely overlapping with tha', thoroughly covered in numerous past studies of heavy-ion reactions at lower bombarding energies, where the IMF abundance was apparently too low to be measurable in non-dedicated experiments ${ }^{8}$.

The experiment was performed at the National Superconducting Cyclotron Laboratory 
of the Michigan State University. A beam of $28.2 \mathrm{MeV} /$ nucleon ${ }^{136} \mathrm{Xe}$ ions from the K1200 cyclotron was focused on a self-supporting $1.5 \mathrm{mg} / \mathrm{cm}^{2}{ }^{209} \mathrm{Bi}$ target, placed in the center of the complex detector setup providing a virtually $4 \pi$ angular coverage for neutrons, light, and intermediate-mass charged reaction products. Neutrons were detected using the Rochester neutron multiplicity meter (NMM) operated in a geometrical configuration and at electronical settings ensuring a detection efficiency of about $50 \%$ for neutrons emitted from slow-moving sources. Light charged particles (LCP) and IMF's were detected using the Washington University Dwarf Array ${ }^{9}$, configured with 95 plastic-CsI phoswich detectors to cover about $80 \%$ of $4 \pi$. Depending on each individual detector, this array provided for an atomic number identification from $Z=1$ up to $Z=14-25$, with an energy threshold of approximately $4 \mathrm{MeV} /$ nucleon. In order to detect additionally massive products, a multielement position-sensitive silicon detector telescope was placed at a forward angle, covering an angular range from $3.0^{\circ}$ to $5.6^{\circ}$. This telescope allowed one to detect and identify, with a low energy threshold, not only massive products but any products with atomic numbers $1 \leq Z \leq 54$.

Figs. 1 through 3 show experimental results for IMF's produced in peripheral collisions, selected either by imposing the condition that a massive projectile-like fragment (PLF) is detected at forward angles or that the associated neutron multiplicities are low to moderate. Analysis of the data from the present experiment has not only shown clearly that the presence of a massive PLF survivor is a signature of moderate dissipation of kinetic energy, but also that the atomic number $Z_{P L F}$ of this (serondary) PLF is sharply correlated with kinetic energy dissipation (ref Shawn). This observable $Z_{P L F}$ may, hence, serve as an alternative measure of the degree of energy damping which is assumed to be related with the impact parameter. Since these PLF events exhaust almost the entire reaction cross section, the $Z_{P L F}$ observable covers almost the entire range of impact parameters.

Fig. 1 compares the general trends in the measured multiplicities of neutrons $\left(M_{n}\right)$, LCP's $\left(M_{L C P}\right)$ and IMF's $\left(M_{I M F}\right)$ as functions of the atomic number $Z_{P L F}$ of the massive PLF survivor. As indicated by the approximate excitation energy scale displayed on the 
top axis, the data in this figure cover an excitation energy range largely overlapping with that accessible at bombdrding energies of only a few $\mathrm{MeV} /$ nucleon above the interaction barrier. This excitation energy scale was established, based on statistical-model calculations of the neutron multiplicitie as a function of excitation energy, assuming a binary reaction scenario $^{10,7}$. As seen in Fig. 1, all three multiplicities increase with decreasing $Z_{P L F}$ or increasing total excitation energy in a very similar fashion. The IMF multiplicities are seen in this figure to be lower than those of the coincident neutrons and LCP's. The former multiplicities are, however, still significantly higher than predicted by statisticalmodel calculations or than those actually reported for the same excitation energy range at lower bombarding energies ${ }^{8}$. Fig. 1 shows, hence, that at $28 \mathrm{MeV} /$ nucleon, an overproduction of IMF's occurs not only in central but also in relatively peripheral collisions, where it has most likely a non-statistical, dynamical origin.

The dynamical character of IMF production is more clearly inferred from Fig. 2, where Galilei-invariant velocity distributions are plotted separately for coincident IMF's $(4 \leq Z \leq$ 6 - panels on right) and $\alpha$-particles (panels on left), for two different bins in $Z_{P L F}$, i.e., for different dissipated energies. As seen from this figure, the IMF yield is dominated by a single peak located at velocities intermediate between those of PLF and TLF, in the same events in which $\alpha$-particles are emitted mostly sequentially and isotropically from two heavy, distinctly different (PLF and TLF) sources, as signaled by the presence of well-defined "Coulomb rings". Such an emission pattern shows clearly that most IMF's do not receive any noticeable net Coulomb boost from the system and, hence, are most likely produced dynamically in the interface region between the two hot fragments seen to evaporate $\alpha$ particles at a later stage. Such an interface or neck is believed to be formed adiabatically between the interacting reaction partners already at lower bombarding energies. An analysis of the massive-fragment phenomenology in the present reaction ${ }^{7}$ has clearly shown that the underlying dynamics of the peripheral collisions at $28 \mathrm{MeV} /$ nucleon is very similar to that known from studies at lower bombarding energies and involves dissipative orbiting or rainbow scattering phenomena. A more evolutionary than instantaneous character of these 
phenomena may allow for the development of a "neck" connecting the reaction partners in the entrance channel when the relative motion has slowed down,. Subsequently, when the fragments separate rapidly, the neck may fail to react adiabatically and snap in two or more locations, whereby a fragment of intermediate mass may be left behind. To verify if the emission pattern of IMF's displayeded in Fig. 2 is compatible with such a dynamical scenario of multiple neck rupture, simple three-body Coulomb-trajectory calculations were performed, assuming that the IMF emerges with a low transversal velocity from the space between the two massive reaction partners. The average asymptotic velocities of IMF's predicted by these calculations were found to agree reasonably well with those observed, thus confirming the viability of such a hypothetical dynamical IMF production scenario.

It is worth stressing that the formation of a classical "fireball" "participant zone" viewed by two spectators, does not seem to provide an alternative scenario for IMF production compatible with the present data. This scenarios does not allow for an excitation of the spectators that is sufficiently high to explain the observed neutron and LCP multiplicities and emission patterns, nor is it consistent with other observed damped-collision-like features ${ }^{7}$ of massive-fragment production. Neither is a classical preequilibrium-type process a viable alternative, because of the complex nature of the IMF's to be "assembled" in the course of the necessary coalescence process. However, in the past, processes have been observed experimentally that are similar to an emission associated with neck rupture considered here. Examples of such reported processes are spontaneous ${ }^{11,12}$ or heavy-ion induced ${ }^{13}$ ternary fission, neck emission of $\alpha$-particles in damped reactions ${ }^{14}$, or the strongly anisotropic IMF emission accompanying ${ }^{3} \mathrm{He}$-induced fission ${ }^{15}$. Also, theoretical models have been advanced in the past to describe instabilities which may possibly lead to multiple neck rupture ${ }^{16,17}$.

It is natural to expect that $\alpha$-particles would also be emitted in a dynamical scenario similar to that in which most of the observed IMF's are generated. However, no experimental verification of such an expectation is possible from the presented data, as in the case of $\alpha$ particles, the respective yield, if any, would be obscured by the "pile-up" peak seen to result from the intersection of the two intense Coulomb rings, associated with sequential emission 
from PLF and TLF, respectively.

As seen from Fig. 2, relatively few IMF's are emitted from the same equilibrated sources that are reflected by the Coulomb rings in the emission patterns of coincident $\alpha$-particles. An increase in the multiplicity of these "evaporative" IMF's with increasing energy dissipation (compare panels on top and bottom right) is in qualitative agreement with phase-space considerations for a statistical emission scenario. A more quantitative assessment of the relative weight of dynamical and statistical components in the overall IMF yield can be accomplished by analyzing the one-dimensional energy spectra of IMF's as functions of energy loss. As shown in Fig. 3, such spectra evolve distinctly with increasing energy dissipation, measured here in terms of associated neutron multiplicity $M_{n}$. For the most peripheral collisions, i.e., the lowest neutron multiplicities, the energy spectrum of IMF's is largely dominated by a low-energy $\left(E_{I M F} \leq 200 \mathrm{MeV}\right)$ component discussed earlier, in the context of Fig. 2, and attributed to the above dynamical emission mechanism. With increasing degree of energy dissipation, a component centered at higher energies appears, which then steadily increases in intensity while merging with the low-energy component. This highenergy component has signatures characteristic of IMF evaporation from the moving PLF. For the largest observed $M_{n}$, the two components are no longer clearly distinguishable in the one-dimensional energy spectrum of Fig. 3. IMF energy spectra at forward angles $\left(\Theta_{\text {lab }} \leq\right.$ $32^{\circ}$ ) were analyzed in terms of statistical evaporation from three moving sources, PLF, TLF, and an effective source simulating the dynamical emission process, in order to derive crude quantitative characteristics of such effective sources. In such an analysis, the PLF and TLF sources were assigned velocities as derived from the PLF telescope data, and the "dynamical" source was assumed to be moving with the center of mass of the whole system. The best fit for the energy spectra of IMF's with $4 \leq \mathrm{Z}_{I M F} \leq 6$ at low to intermediate degrees of energy dissipation was obtained when a Coulomb barrier of approximately $5 \mathrm{MeV}$ and a slope parameter of approximately $30 \mathrm{MeV}$ were assumed for the effective dynamical component. Furthermore, this dynamical component was found to be non-isotropic in the center-of-mass reference frame, as would be qualitatively expected for Coulomb-focused neck remnants, 
with a maximum yield at angles around $\Theta_{l a b}=60^{\circ}$.

In a different quantitative analysis of the IMF yield, it was found that the atomic number distributions of IMF's are of the exponential form $\mathrm{d} \sigma / \mathrm{d} Z \propto \exp \left(-Z / Z_{\mathrm{o}}\right)$, with a slope parameter $Z_{\circ}$ increasing with increasing degree of energy dissipation and decreasing with increasing emission angles. It may be speculated that such dependence of the slope parameter $Z_{\circ}$ on the dissipated energy is a further reflection of the fact that IMF's result from the rupture of the neck. A more massive neck formed in more dissipative collisions can be expected to favor release of IMF's with higher atomic numbers, which would lead to the observed correlations between the slope parameter and dissipated energy.

In conclusion, an analysis of the IMF yield from peripheral collsions of ${ }^{209} \mathrm{Bi}$ with ${ }^{136} \mathrm{Xe}$ at $E_{\text {lab }} / A=28.2 \mathrm{MeV}$ has shown that these fragments are produced to a significant extent in non-equilibrium or dynamical processes and in geometrical locations at the intermediate system which are characterized by relatively low gradients of the Coulomb potential. A simple production scenario, in which IMF's are remnants of a multiple rupture of the neck formed transiently between the interacting reaction partners, appears to be compatible with the data analyzed. Such neck rupture processes have been observed in experiments with macroscopic water droplets ${ }^{18}$, where the effect is due to the development of Rayleigh instabilities. The possibility to study such macroscopic behavior of nuclear matter in intermediate-energy heavy-ion collisions under controlled experimental conditions represents unexpected and exciting opportunities. However, more stringent theoretical modelling and experimental investigations are necessary in order to establish the pertinent features of the different possible IMF production mechanisms with a sufficient degree of certainty.

\section{ACKNOWLEDGMENTS}

This work was supported by the U.S. Department of Energy grants No. DE-FG0288ER40414 (University of Rochester), DE-FG02-87ER403160 and DE-FG02-88ER40406 (Washington University), and by the National Science Foundation grants No. PHY-89- 
13815 (NSCL). 


\section{REFERENCES}

*. On leave from CRN Strasbourg, BP 20 CRO, F-67037 Strasbourg, France.

†. Present address: GANIL, BP 5027, F-14021 Caen, France.

ł. On leave from Instituto de Fisica da UFRJ, Rio de Janeiro, Brazil.

§. Present address: Indiana University, Department of Chemistry, Bloomington, IN 42405, USA.

1. C. K. Gelbke and D. H. Boal, Prog. Part. Nucl. Phys. 19, 33 (1987), and references therein.

2. B. Borderie, et al.,Ann. Phys. (Fr) Vol.15 (1990), and ref. therein.

3. W. A. Friedman, Phys. Rev. Lett. 60, 2125 (1988).

4. W. A. Friedman, Phys. Rev. C42, 667 (1990).

5. A. Sokolov, Thèses de l'Université Paris 6, GANIL T90-01 (1990) unpublished.

6. J. L. Wile, et al., Phys. Lett. B264, 26 (1991).

7. S.P. Baldwin et al.,Proc. of the Workshop on Nuclear Dynamics, Key West (1993).

8. W. U. Schröder and J. R. Huizenga, in Treatise in Heavy-Ion Science, vol. 2, D. A. Bromley, Editor, Plenum Press, New York and London, 113, (1984), and references therein.

9. D.W. Stracener et al., Nucl. Instr. and Meth. A294, 485 (1990).

10. B. Lott, et al., Phys. Rev. Lett. 68, 3141 (1992).

11. R. Vandenbosch and J.R. Huizenga, in Nuclear Fisssion, Academic Press, New York (1973), and ref. therein.

12. J.P. Theobald et al., Nucl. Phys. A502, 361c (1989), and ref. therein. 
13. J.P. Kosky et al., Phys. Lett. 133B, 153 (1983).

14. W. W. Wilcke et al., Phys. Rev. Lett. 51, 99 (1983).

15. D.E. Fields et al., Phys. Rev. Lett. 69, 3713 (1992).

16. U. Brosa, et al., Z. Phys. A310, 177 (1983).

17. L. G. Moretto, et al., Phys. Rev. Lett. 69, 1884 (1992).

18. P. R. Brazier-Smith, et al., Proc. R. Soc. Lond. A326, 393 (1972) 


\section{FIGURES}

Fig. 1. Multiplicities of neutrons $\left(M_{n}\right)$, light charged particles $\left(M_{L C P}\right)$, and intermediate mass fragments $\left(M_{I M F}\right)$ from the ${ }^{209} \mathrm{Bi}+{ }^{136} \mathrm{Xe}$ reaction at $E_{\text {lab }} / \mathrm{A}=8.2 \mathrm{MeV} /$ nucleon as functions of the atomic number $Z_{P L F}$ of the projectile-like fragment.

Fig. 2. Galilei-invariant velocity distributions of coincident $\alpha$-particles (left-hand-side panels) and IMF's (right-hand-side panels) for two different bins in atomic number $Z_{P L F}$ of the coincident massive fragment, indicated in the panels.

Fig. 3. Energy spectra of IMF's with $4 \leq Z_{I M F} \leq 6$ measured at $\theta_{l a b}=14^{\circ}$, for different bins in the associated neutron multiplicity. 


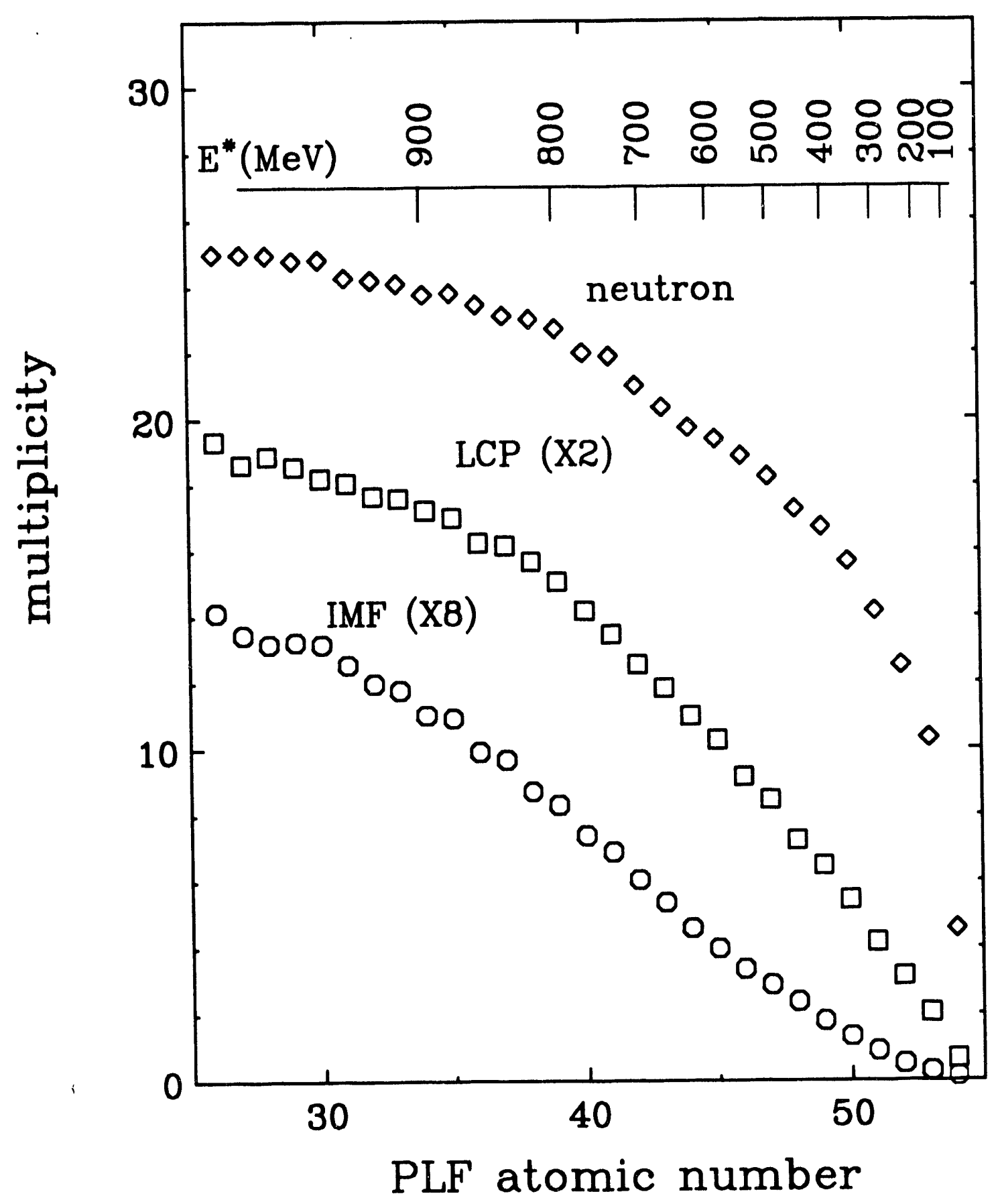

Figure 1 


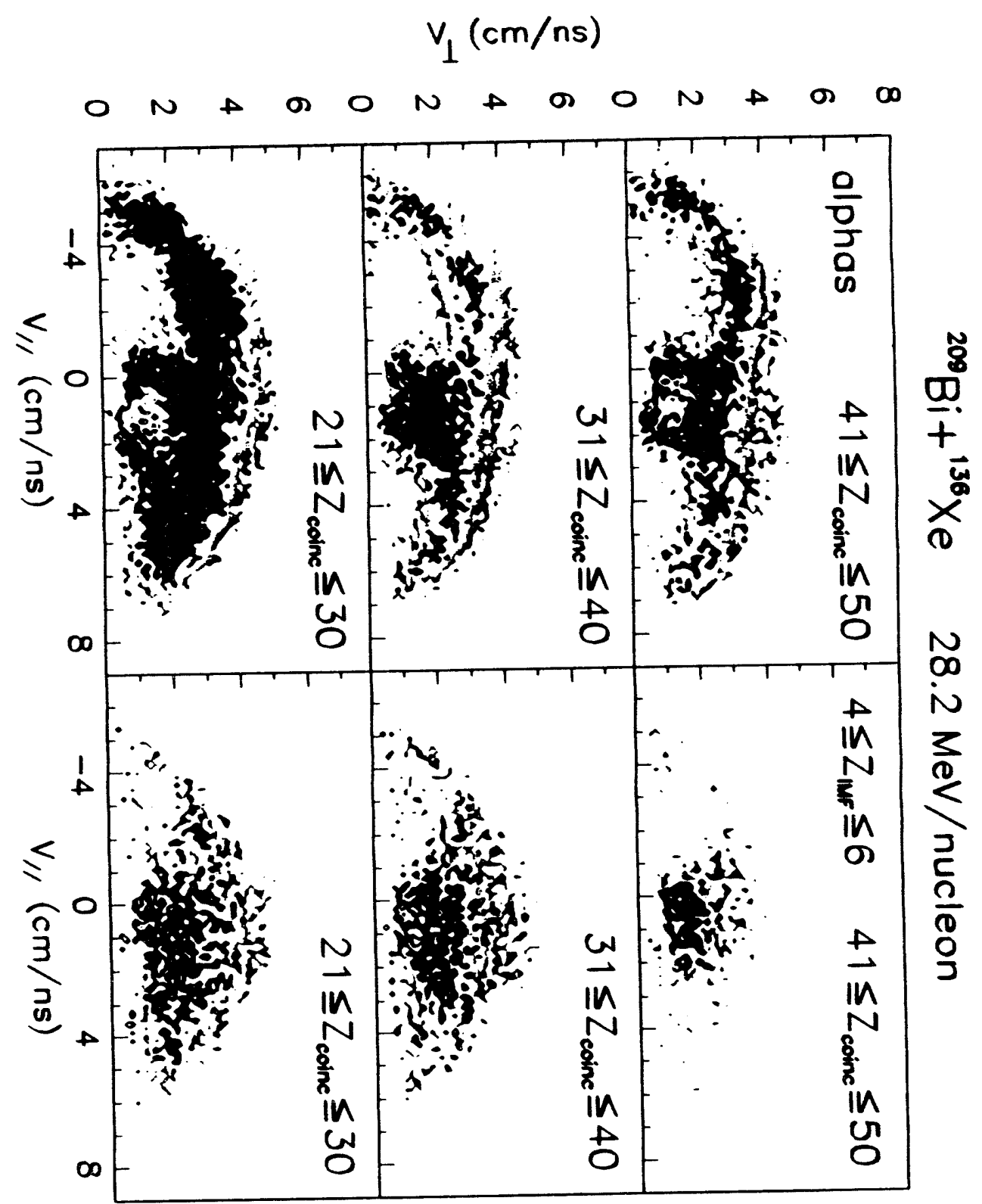




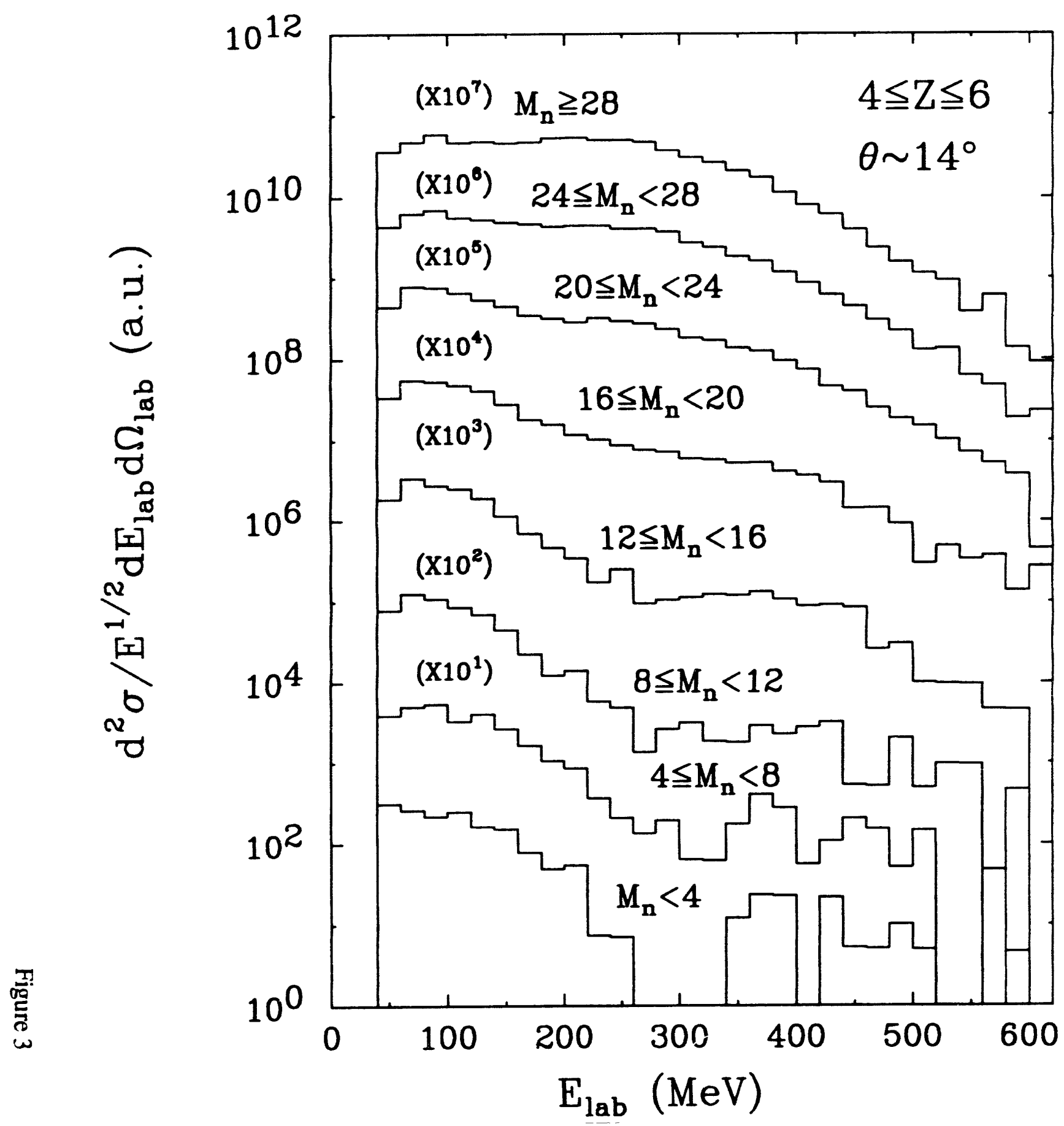




\author{
S. P. Baldwin, D. Pade*, B. M. Quednau ${ }^{\dagger}$, W. U. Schröder, J. Töke \\ Department of Chemistry and Nuclear Structure Research Laboratory, \\ University of Rochester, Rochester, N.Y. 14627
}

\begin{abstract}
A gadolinium-loaded liquid-scintillator neutron multiplicity meter has been designed and built for heavy-ion reaction studies at intermediate bombarding energies. The detector design and performance are discussed along with methods of its energy and efficiency calibration and methods of processing of signal streams generated by the detector in response to multi-neutron reaction events. The detector has been successfully used in experiments at both low and intermediate bombarding energies.
\end{abstract}

\title{
I. INTRODUCTION
}

Neutron detection is one of the important tools in heavy ion reaction studies, allowing one to gain insights into both the interaction dynamics and the properties of the nuclear matter or properties of systems produced in such reactions over a broad range of excitation energies and angular momenta. Along with measurements of energy spectra, angular distributions, or various correlations involving individual neutrons, a high-efficiency measurement of the numbers of neutrons emitted in reaction events, i.e., neutron multiplicities, proved to be a valuable tool in studying heat content of nuclear systems transiently formed in such collisions [1]. Commonly, the detectors used for the neutron multiplicity measurements, called neutron multiplicity meters (NMM), use gadolinium loaded liquid scintillator as the active agent. Furthermore, to ensure high detection efficiency, such detectors typically have rather large 
volumes and are operated in a geometrical configuration providing virtually a $4 \pi$ solid angle coverage for neutrons emitted from the transient system of interest.

The present paper provides a detailed description of the 960-liter volume gadolinium loaded liquid scintillator NMM designed and built at the University of Rochester, and successfully used in heavy-ion reaction studies both at low and intermediate bombarding energies. the principle of operation of the detector is presented in Section II. Its mechanical design is discussed in Section III. Section IV discusses results of theoretical simulation calculations for the response of the detector expected under various reaction scenarios, which served also largely as a guide during the design stage. Techniques of processing the streams of electronic signals generated by the detector in response to multi-neutron events and techniques of event acquisition used in connection with the NMM are presented in Section V, while methods and results of the efficiency and energy calibration are discussed in Section VI.

\section{PRINCIPLE OF OPERATION}

A neutron multiplicity meter is an instrument to count the number of neutrons simultaneously injected into the active volume of the detector consisting of Gd-loaded liquid scintillator. Through the mechanisms of interaction of neutrons with the active liquid, the NMM generates a randomly delayed response to each neutron, thereby allowing the associated electronics to process the detector response to each neutron individually. In addition, the detector generates a prompt response signal to neutrons and $\boldsymbol{\gamma}$-rays, carrying information on the energy of these neutrons and $\gamma$-rays. The mechanisms in which these response signals are generated are discussed below.

Neutrons entering the detector volume are first thermalized through the scattering off the nuclei of the constituent atoms of the scintillator fluid. Primarily, the neutrons interact with the hydrogen, carbon, and oxygen nuclei through elastic and inelastic scattering, although several nuclear reactions also have non-negligible probabilities. The cross sections of some 
of the probable types of interactions are shown in Fig. 1 [2]. As seen from this figure, the cross sections of the various processes are strongly dependent on the neutron energy, with elastic scattering on protons and carbon nuclei being the dominant processes for neutrons with kinetic energies below $60 \mathrm{MeV}$. The $\mathrm{C}(\mathrm{n}, \alpha) \mathrm{Be}$ and $\mathrm{C}(\mathrm{n}, \mathrm{p}) \mathrm{B}$ reactions, important near 10 $\mathrm{MeV}$ and above $20 \mathrm{MeV}$, respectively, actually remove neutrons from the chain of interactions leading to the delayed response, critical for counting the neutron multiplicity.

The moderation process occurs on the time scale of a few tens of nanoseconds, producing charged recoiling nuclei, predominantly protons, which excite the luminescent material, ultimately producing a scintillation with intensity proportional to the kinetic energy of the neutrons initially injected into the scintillator volume. The corresponding electric current produced by the photomultiplier tube (PMT) viewing the active volume is approximately proportional to the light output of the scintillator fluid, and this signal occurs promptly with the reaction event in the target ("prompt response").

The thermalized neutrons diffuse through the scintillator fluid in a random-walk-like process, until they are captured by atomic nuclei, and most likely by the nuclei of the gadolinium atoms, or until they escape the scintillator volume, thereby generating no further detector responses. ${ }^{155} \mathrm{Gd}$ and ${ }^{157} \mathrm{Gd}$ have large cross sections [3], $6.1 \times 10^{4} \mathrm{~b}$ and $2.5 \times 10^{6} \mathrm{~b}$, respectively, for the capture of thermal neutrons. The capture of neutrons by Gd occurs randomly in time, with the characteristic probability distribution shown in Fig. 2. This neutron capture time distribution is dependent both on the moderation strength, $\alpha$, of the scintillator fluid and on the concentration, $\beta$, of $\mathrm{Gd}$ atoms in the scintillator, and can be expressed in terms of these parameters as

$$
f(t)=e^{\alpha t}\{t(\beta-\alpha)-1\}+e^{-\beta t},
$$

where $t$ denotes time lapse from the instance of injection. In typical applications of the NMM, the most probable time of capture of a neutron by Gd is of the order of $10 \mu$ s, i.e., well within the time-resolving capabilities of the electronical circuits.

After the capture of a neutron by a Gd nucleus, the excited Gd decays by emitting a 
cascade of $\boldsymbol{\gamma}$-rays. There are two possible cascades with total energy $[4,5]$ of either 7.94 or 8.34 MeV, each consisting of a few $\gamma$-rays, for ${ }^{158} \mathrm{Gd}$ and ${ }^{156} \mathrm{Gd}$, respectively. The $\gamma$-rays from the deexcitation cascades interact then with the scintillator fluid, generating light flashes (in the blue region) which are detected by the photomultiplier tubes viewing the active volume of the NMM. This detection process of the $\gamma$-rays has a time scale of a fraction of a nanosecond, negligible on the time scale of the capture time probability distribution. The above light flash generated by the capture $\gamma$-rays serves as a delayed response of the NMM to the neutrons. The neutron multiplicity is measured by counting the number of flashes over a time interval of the order of $50-100 \mu \mathrm{s}$, following the reaction event, encompassing most of the capture time distribution.

In addition to the delayed response to neutrons, the scintillator generates prompt responses both to the reaction $\gamma$-rays and the neutrons. The latter signal reflects the kinetic energy of neutrons converted on the fast time scale of the moderation process into the kinetic energy of recoiling charged nuclei. This prompt detector response carries, hence, information about the total kinetic energy of all neutrons that have entered the active volume of the detector in each event and the energy of all reaction $\gamma$-rays. In past applications of the NMM, however, this valuable information carried by the prompt signal was not fully appreciated.

\section{MECHANICAL DESIGN}

A computer-rendering of the Rochester NMM is displayed in Fig. 3. Fig. 4 shows a detailed vertical section view of the detector with the scattering chamber, vessel, window attachment flanges, and electrical feedthrough flanges. The internal reaction chamber and the target site are exposed in Fig. 3 through simulated cut-outs in the NMM outer shell and in the reaction chamber walls. The scintillator is viewed by 14 photomultiplier tubes (PMT) housed inside canisters mounted to the outer wall of the vessel. The vessel is constructed from two 19" high right-truncated cones welded to the ends of a 48" diameter and 19" high 
cylinder. The axes of the cones and cylinder are perpendicular to the beam direction. The vessel is fabricated from $1 / 8$ " thick carbon steel [6-8].

The reaction chamber fits inside the $253 \mathrm{gal}$. vessel with an axis parallel to the beam direction. The components of the scattering chamber are fabricated from 304 stainless steel. The chamber, shown in Fig. 5, is essentially cylindrical in design with hemispherical and conical transition pieces. The entrance to the chamber is a 4" diameter tube with $1 / 16$ " thick walls. A $1 / 16$ " thick hemisphere with a 6 " radius centered in the vessel serves as a transition to a $121 / 8$ " diameter tube with a $1 / 8$ " thick wall. The larger tube is welded to a cone with a vertex at the center of the vessel with a 19 degree opening. The conical transition piece is converted to 4" diameter beam tube through a flange, which contains various feedthroughs on its circumference. The reaction chamber is modular for ease of transportation, assembly, and the possibility of accommodating various charged product detector arrays [9].

Fourteen flanges welded to the vessel hold $3 / 8$ " thick windows through which the PMT's view the scintillator volume. Fig. 6 shows a detailed vertical section of a typical PMT housing assembly with window. The windows were fabricated from BK-7 glass which has a similar index of refraction, of approximately 1.5 , as the fluid and PMT front face. Having the PMT isolated from the scintillator has advantages over a direct contact of the PMT with the scintillator fluid. A malfunctioning PMT can be replaced without draining the fluid from the vessel, and chances of physical damage to a PMT are reduced. Most importantly, since no liquid-air seal is needed at the scintillator-PMT interface, the possibility of a leak is virtually eliminated. All fluid seals are made with viton O-rings or Teflon gaskets, since the scintillator base, 1,2,4-trimethylbenzene, reacts with Buna seals, copper, brass, carbon steel, and various plastics.

In order to improve the light collection and to protect the walls (carbon steel) of the detector from the corrosive action of the scintillator fluid, the inner walls of the NMM were coated with a diffusely reflecting paint. A titanium-dioxide-pigmented, epoxy-based paint (Bicron 622A) was chosen for the Rochester NMM, based on its compatibility with the scintillator fluid (Bicron 521). In order to assure long-lasting paint adhesion, the surface 
has been sandblasted and freed of grease prior to coating, following the manufacturer's recommendations.

As seen from equation 1, the capture time probability is dependent on the concentration of the gadolinium atoms in the scintillator. The Gd concentration is usually optimized for the anticipated neutron multiplicities. There are three main interrelated aspects to be considered for a proper choice of a Gd concentration, the neutron detection efficiency, the capture time distribution, and the time between two sequential neutron captures. The neutron detection efficiency increases with the Gd concentration, but increasing the Gd concentration shortens the decay constant of the capture time probability distribution, thus decreasing the characteristic time between neutron captures and increasing the probability that two successive captures will be unresolvable by the electronics ("pileup").

The Rochester NMM uses a scintillator (Bicron-521) with a solvent base of 1,2,4trimethylbenzene loaded with $0.2 \%$ wt. natural Gd. The response of the scintillator to $\gamma$-rays was measured with a 2 -liter test cell. Fig. 7 shows that the two Compton edges in the energy spectrum of the $\gamma$-rays from a ${ }^{22} \mathrm{Na}$ source are well resolved. The scintillator has a bulk light attenuation length larger than 4 meters. Through the addition of a luminescent solute to the scintillator fluid, the maximum intensity in the emission spectrum, Fig. 8 , is wavelength-shifted to $424 \mathrm{~nm}$ to match the spectral response of the PMT [10], shown in Fig. 9. Other physical properties of the scintillator fluid are given in Table 1.

The scintillator fluid is transferred to the NMM tank in a closed system to minimize contamination of the fluid with oxygen and water and to limit leakage of the vapors to the surrounding area. The system is first purged with nitrogen, and then fluid transfer is achieved by pressing the fluid out of the storage drums to the tank using nitrogen gas. Once the NMM is filled, all vents are closed and the pressure within the vessel is monitored. The NMM tank is equipped with an expansion vessel mounted on its top to counter change in volume of the liquid due to fluctuations in the ambient room temperature. 


\section{COMPUTER SIMULATIONS OF THE RESPONSE}

The response of the Rochester NMM was modeled [11] with a Monte Carlo simulation code. A modified version of the code was used [12], which allows one to treat a wide variety of detector shapes and accounts for the anisotropy of the neutron angular distributions for scattering on protons and carbon nuclei and the angular dependence of Compton Scattering. This modified code is well suited for calculating the response of the NMM to neutrons from intermediate-energy heavy-ion reactions.

Plotted in Fig. 10 is the calculated detector efficiency versus neutron energy, for neutrons isotropically emitted from a stationary source positioned at the target position of several neutron multiplicity meters in operation. The response of the Rochester NMM (circles) is compared to that of the detector ORION at GANIL (squares), and the NMM at HMI-Berlin (diamonds). The Rochester NMM has an approximately $85 \%$ and $20 \%$ efficiency for $5 \mathrm{MeV}$ (slow) and $40 \mathrm{MeV}$ (fast) neutrons, respectively. The efficiencies for counting slow neutrons are comparable for all four NMM's, but the Rochester NMM, equipped with a specially designed scattering chamber, has the lowest efficiency, relative to the other NMM's, for detecting energetic neutrons emitted at forward angles, $|\Theta| \leq 19^{\circ}$.

The response function of the Rochester NMM has been specifically optimized for counting neutrons emitted from a slow-moving hot source, such as a compound nucleus formed in a fusion reaction or a target-like fragment (TLF) from a damped reaction. There is a high efficiency for detecting and counting neutrons from such sources, while neutrons from fast sources, such as projectile-like fragments (PLF), have a high probability for escaping detection. Thus, the Rochester NMM has a high sensitivity to slow neutrons as compared to fast neutrons.

The positions at which neutrons were captured, as given by the simulation code, are plotted in Fig. 11 to illustrate the sensitivities of the Rochester NMM to slow and fast neutrons. The neutron capture points in a $9 \mathrm{~cm}$ thick equatorial slice of the detector are plotted with the ordinate of the plot coinciding with the beam axis. Panels A and B display 
the capture positions of neutrons isotropically emitted from excited PLF and TLF sources, respectively, produced in a damped heavy-ion reaction with a total kinetic energy loss of $500 \mathrm{MeV}$. The relative intensities in the two panels reflects the efficiencies of the detector for counting neutrons emitted from the TLF and PLF sources. The Rochester NMM has approximate efficiencies of $70-80 \%$ and $5-15 \%$ for counting neutrons emitted from TLF and PLF sources, respectively. The efficiencies depend on the threshold set on the light output signal, which is typically adjusted to $1 \mathrm{MeV}$ equivalent $\boldsymbol{\gamma}$-ray energy.

Fig. 12 shows the response function of the NMM calculated with a Monte Carlo simulation code [13], for damped reaction the scenario characterized by two moving sources of neutrons, a projectile-like fragment (PLF) and a target-like fragment (TLF). The dashed and dot-dashed lines represent the response of the NMM to neutrons emitted from the PLF and TLF, respectively, and the solid line denotes the overall efficiency of the detector. The calculation is similar to a statistical-model calculation and includes charged-particle emission and recoil effects. Such a response function can be used to correct the measured multiplicities after background subtraction.

\section{SIGNAL PROCESSING AND ACQUISITION TECHNIQUES}

The NMM is equipped with Philips XP-2041, 14-stage fast photomultipliers. Modified Thorn EMI TB1106 active bases are used to apply positive high voltage to the photomultiplier dynodes. The bases are modified [14] so that the anode signal can drive a 50-ohm cable, thus eliminating the need for a high-gain amplifier. This is accomplished with a pnp-type transistor operated in the emitter follower mode. The transistor is biased using the potential drop between the anode and 14th dynode to operate at a quiescent collector current of $1 \mathrm{~mA}$ common with that of the voltage divider chain of the base. A non-inverted emitter "image" of the anode signal is extracted and mixed with the other thirteen anode signals.

A schematic representation of the typical signal processing circuitry is shown in Fig. 13. Two signals are taken from each PMT base, the anode signal and the signal of the 14th 
dynode. The individual dynode signals of all PMT's are summed together, generating one signal for the total light output of the NMM. Two options for processing the anode signals have been utilized for this detector. One technique, option $A$, requires an amplifier and leading-edge discriminator channel for each of the PMT anode timing signals. Alternatively, in option B, the anode signals are mixed and a discriminator level set on the resulting sum signal. The former technique retains the information on which of the PMT's generate pulses for a given event, whereas the latter technique allows a threshold to be set on the total light output. For each light flash produced by the scintillator, two usable signals are generated, an analog signal from the dynode, containing information on the energy deposited in the scintillator by the radiation, and a logic timing signal.

Typically, the Rochester NMM is operated in coincidence with other detectors defining a reaction event. A block diagram of this typical set up is shown in Fig. 14. The logic timing signal from the NMM is used to provide the stop signals for the event handler and for the coincidence circuit, which will eventually start the event handler. The start for the event handler is typically generated from a coincidence between the timing signals of a "trigger" fragment detector and the prompt response of the NMM. This start signal is delayed approximately $200 \mathrm{~ns}$ in order to not mis-identify the corresponding prompt-stop signal as a neutron. Additional start signals are generated randomly to initiate a background measurement as shown in Fig. 14.

An event handler [15] built to CAMAC standards determines the neutron multiplicity of an event and records the times between the event start and each NMM logic signal associated with the capture of a neutron. Measuring the times of capture provides the option to optimize the NMM efficiency by varying the counting-gate length off line via software. Also, corrections can be made for discriminator double-triggering by monitoring the times between successive captures. The event handler generates an internal counting gate which defines the duration of the multiplicity measurement. Fig. 14 illustrates the generation of event handler input signals. The start for the event handler is typically defined by a coincidence between the logic timing signals of the NMM and a silicon or CsI detector measuring charged reaction 
products, for example. The time sequence of the physical processes and signal processing stages are outlined in Fig. 15 for typical operation of the Rochester NMM. The projectiletarget collision occurs at time zero when neutrons and $\gamma$-rays are instantaneously injected into the active volume of the NMM. The attenuation of the $\gamma$-rays produces a "prompt" response within a few nanoseconds. In addition, the moderation of the injected neutrons contributes to the prompt response, but on a somewhat longer time scale.

There are various contributions to the average time required to record each event. The NMM has an intrinsic dead time on the order of several tens of microseconds to register the neutrons instantaneously injected into the scintillator volume at the time of the reaction. Presently, the Rochester NMM uses a 128-microsecond counting period. The frequency of background multiplicity measurements increases the time during which a rection events can not be processed. Typically, a background multiplicity measurement is made a few hundred microseconds after a valid reaction event has been registered, using the same 128 microsecond counting period as for the neutron events. The frequency of thes: measurements depends on the acceptable event dead time for each experiment. The detector has been operated in modes with a background multiplicity measurement for each event and for every 32nd or 64th event. For the latter mode, the event is tagged, to identify it as a background measurement. The computer read-out of the data stored in the event handler depends solely on the amount of information one desires to record for each event.

Other modes of NMM setup allow measurements of the light output generated in the detector by $\gamma$-ray sources, ${ }^{252} \mathrm{Cf}$ spontaneous fission neutron sources, and by the deexcitation of a Gd nucleus after neutron capture. The light output response of the detector to $\gamma$-ray sources is measured by removing the requirement of a coincidence between a trigger detector and the NMM. The NMM light output for the ${ }^{252} \mathrm{Cf}$ spontaneous fission neutrons is measured by requiring a coincidence between a fission fragment and the NMM prompt signal. The scintillation light output spectrum for the deexcitation of $\mathrm{Gd}$ nuclei after neutron capture can be measured by identifying the analog dynode signal corresponding to the capture of a neutron. The analog signal is identified by coincidence between the event handler start 
signal, delayed by a few microseconds and stretched to $30 \mu \mathrm{s}$, and an event handler stop signal. The NMM responses that satisfy this coincidence requirement result approximately $95 \%$ of the time from the capture of a neutron.

The setup of the NMM electronics consists mainly in gain-matching of the PMT's and in adjusting the electronic threshold set on the PMT anode signals. Since PMT's are symmetrically mounted on the vessel and all view approximately equal volumes of scintillator, the individual PMT's should have similar responses to a source positioned at the center of the NMM. Hence, in the gain matching procedure, the light output spectrum for the prompt response of the NMM for ${ }^{252} \mathrm{Cf}$ spontaneous fission neutrons is collected for each PMT. The voltage applied to the individual PMT is adjusted to position the peak at a common channel in the prompt response light output spectrum. The discriminator thresholds on the individual anode signals are then set at the same channel in their corresponding light output spectrum, generated from the dynode signal.

\section{EFFICIENCY AND ENERGY CALIBRATION}

A NMM prompt response spectrum is displayed in the next series of figures. Fig. 16 shows the raw and background-subtracted light output spectra, collected for various $\gamma$-ray sources. The NMM response corresponds to the full energy of all $\gamma$-rays simultaneously emitted. Fig. 17 shows the light output spectrum for the deexcitation of Gd nuclei after capturing neutrons emitted from a ${ }^{252} \mathrm{Cf}$ source. The spectra shown in Figs. 16 and 17, can be used to calibrate the light output to absolute $\gamma$ - ray energy, taking into account the NMM efficiency and solid-angle corrections. The result of such a calibration is shown in Fig. 18 where $\gamma$-ray energy is plotted vs. light output (channels). The prompt NMM light output response for ${ }^{252} \mathrm{Cf}$ fission neutrons is shown in Fig. 19. The prompt light is produced from two different processes, the attenuation of $\gamma$-rays and the thermalization of neutrons. Therefore, to interpret the prompt signal precisely, the detector's response function for neutrons must also be determined, or the energy spectrum of the neutrons must 
be known. This is in practice, often not necessary, because the energy dissipated in the scintillator by neutrons is usually much larger than the energy deposited by $\gamma$-rays.

The efficiency of the NMM is determined from a measurement of the neutron multiplicity distribution for spontaneous fission of ${ }^{252} \mathrm{Cf}$, which has a precisely known average value of $\left\langle\mathrm{m}_{n}\right\rangle=3.773$. This efficiency can be compared to the predictions of the simulation code, thereby fixing the detection threshold and simulated NMM response function. Typically, the NMM is operated with a threshold on $\gamma$-ray energy of $1-2 \mathrm{MeV}$.

\section{SUMMARY}

The Rochester NMM has been used in several experiments, including studies of the ${ }^{209} \mathrm{Bi}$ $+{ }^{136} \mathrm{Xe}$ reaction at $\mathrm{E}_{l a b} / \mathrm{A}=28 \mathrm{MeV}[16]$ and studies of ${ }^{118,124} \mathrm{Sn}+{ }^{32} \mathrm{~S}$ reactions at $\mathrm{E}_{\text {lab }}$ $=130-180 \mathrm{MeV}[17]$. In the $\mathrm{Bi}+\mathrm{Xe}$ experiment both the neutron multiplicity and the prompt response were recorded. Both these observables have been used as a measure of the energy dissipated in the reactions and as tools in determining the collision dynamics. The Rochester NMM was used to measure the neutron multiplicity in the Sn + S systems. The results of these measurements were then used to make comparisons to the predictions of statistical model evaporation codes.

The design, construction, and operation of a medium-volume NMM for measuring neutron multiplicities in both intermediate-energy heavy-ion reactions and fusion reactions has been discussed. The detector is specifically designed with a high sensitivity to neutrons emitted from a slow nucleus, i.e. speeds less than or equal to approximately $1 \mathrm{~cm} / \mathrm{ns}$. Thus the Rochester NMM is capable of selectively measuring the heat content of slow excited remnants of nuclear collisions, for example, TLF and fusion compound nuclei.

In addition, information about the total kinetic energy of the neutrons and energy of $\gamma$-rays interacting with the scintillation fluid is obtained from the NMM prompt response. A preliminary calibration of the prompt response to $\gamma$-ray energy is obtained using known $\gamma$-ray sources and $\gamma$-rays produced in the capture of thermal neutrons by a Gd nucleus. 
Interpretation of the prompt response in terms of neutron kinetic energy is still under investigation.

\section{ACKNOWLEDGEMENTS}

Prof. R. C. Block from Rensselaer Polytechnic Institute donated the vessel for the Rochesic: NMM. This work was supported by the U.S. Department of Energy under Grant No. DE-FG02-88ER40414. 


\section{REFERENCES}

* Present address: Dornier GmbH, Friedrichshafen, Germany.

${ }^{\dagger}$ Present address: GANIL, BP 5027, F-14021 Caen, France.

[1] D. Pade et al., Phys. Rev. C 43 (1991) 1288-97.

[2] J. A. Strong et al., Nucl. Instr. and Meth. 156 (1978) 411-23.

[3] U. Jahnke et al., Lecture Notes in Physics 178 (1983) 179.

[4] M. A. Lee, Nuclear Data Sheets 56 (1989) 199.

[5] R. G. Helmer, Nuclear Data Sheets 65 (1992) 65.

[6] R. C. Block et al., in Proceedings of the Symposium on Neutron Time-of-Flight Methods, Saclay, France, 1961, edited by J. Spaepen (EURATOM Brussels, 1961) p. 203.

[7] R. W. Hockenbury et al., Phys. Rev. 178 (1969) 1746-69.

[8] T. J. King and R. C. Block, Nuc. Phys. A138 (1969) 556-76.

[9] D. W. Stracener et al., Nucl. Instr. and Meth. A294 (1990) 485-503.

[10] Amperex Data Handbook, Electron tubes, Part 9, March 1978, Amperex Electronic Corporation, Hicksville Division, Hicksville, Long Island, NY 11802.

[11] J. Poitou and C. Signarbieux, Nucl. Instr. and Meth. 114 (1974) 113.

[12] B. M. Quednau and D. Pade, Report DOE/ER/40414-2, (W. U. Schröder and J. R. Huizenga, edts.), (1989) 170.

[13] B. D. Pade, Report DOE/ER/40414-2, (W. U. Schröder and J. R. Huizenga, edts.), (1989) 147.

[14] J. Töke, Report DOE/ER/40414-6, A One Transistor Preamplifier for PMT Anode Signals, (W. U. Schröder, edt.), (1993). 
[15] J. Töke, Report DOE/ER/40414-6, A Five-Channel Multistop TDC/Event Handler for the SuperBall Neutron Multiplicity Meter, (W. U. Schröder, edt.), (1993).

[16] S. P. Baldwin et al., in Advances in Nuclear Dynamics-Proc. of the Workshop on Nuclear Dynamics IX, Dissipative Dynamics of Projectile-Like Fragment Production in the Reaction ${ }^{209} \mathrm{Bi}+{ }^{136} \mathrm{Xe}$ at $E_{\text {lab }} / \mathrm{A}=28.2 \mathrm{MeV}$, Key West, FA, USA, Jan. 31-Feb. 5, 1993, eds. W. Bauer and B. Back (World Scientific, Singapore, 1993).

[17] J. L. Wile et al., Report DOE/ER40414-6, Decay Patterns of Dysprosium Nuclei Produced in ${ }^{32} S+{ }^{118,124} S n$ Fusion Reactions, (W. U. Schröder, edt.), (1993). 


\section{FIGURES}

FIG. 1. Cross sections of most probable interactions between neutrons and the nuclei composing the liquid scintillator used in NMM.

FIG. 2. Calculated capture time probability distribution for a single neutron, using a parameterization derived from experimental data, with values for the parameters $\alpha$ and $\beta$ for the Bicron-521 scintillator fluid used in the Rochester NMM.

FIG. 3. A computer rendering of the Rochester NMM. 14 PMT's are attached to the vessel. Cut-outs simulated in the outer shell of the 253 gallon NMM vessel and in the scattering chamber allow one to view the latter as well as the target site.

FIG. 4. Mechanical drawing of a vertical section view of the Rochester NMM. The modular scattering chamber design is shown along with locations of the ports for attaching PMT's.

FIG. 5. Perspective drawing of the scattering chamber for the Rochester NMM.

FIG. 6. Mechanical drawing of a vertical section of a complete PMT housing assembly. A window (a) is clamped (d) to flange (b) which is welded to the vessel (c). After a PMT is optically coupled to the window (a) and secured (f), the light-tight housing $(e, g)$ is attached to the clamp (d).

FIG. 7. $\gamma$-ray energy spectrum for a ${ }^{22} \mathrm{Na}$ source measured with the BC-521 scintillator fluid in a 2 liter test cell, coated with white teflon. Both the $.511 \mathrm{MeV}$ and $1.2746 \mathrm{MeV}$ Compton edges are clearly seen.

FIG. 8. Emission spectrum of the BC-521 scintillator fluid.

FIG. 9. Spectral sensitivity of the Philips XP2041 PMT 
FIG. 10. Calculated neutron detection efficiency as a function of kinetic energy for various NMM's in operation: ORION-GANIL, squares; HMI-Berlin, diamonds; Rochester NMM, circles. In each calculation, monoenergetic neutrons are assumed isotopically emitted from a hypothetical source located at each detectors target position.

FIG. 11. Capture point distributions for neutrons emitted from PLF and TLF, top and bottom panels, respectively, from a hypothetical heavy-ion collision with approximately $500 \mathrm{MeV}$ energy loss. Both panels represent a horizonal equatorial slice, $9 \mathrm{~cm}$ thick, for the present NMM. The ordinate is the symmetry axis (z-axis) of the scattering chamber and coincides with the beam axis. The relative intensity reflects the efficiency of the NMM detecting neutrons emitted from the PLF and TLF.

FIG. 12. The calculated neutron detection efficiency of the Rochester NMM for neutrons emitted from PLF and TLF sources generated in a simulated heavy-ion reaction. The detection efficiencies for neutrons emitted from the respective sources are plotted at various energy losses, ordinate and abscissa, respectively. The overall neutron detection efficiency and the detection efficiency for neutrons emitted from either a PLF or a TLF, are shown in the figure with solid, dashed, and dot-dashed curves, respectively.

FIG. 13. Two options are outlined for processing the anode and dynode signals extracted from the PMT's of the Rochester NMM. Option A generates a logic timing signal for each PMT on the NMM using a LeCroy 612AM amplifier (Amp) and LeCroy 4608C leading-edge discriminator (Disc) channel. LeCroy 365 AL logic units (LU) impose a coincidence level between the PMT's, ultimately generating a single logic response signal. Option B first mixes $(\Sigma)$ the anode signals and then a threshold is set on the resulting summed anode signal with a Disc. to generate the NMM logic timing signal. The advantages of the two options are discussed in the text. For both options are the dynode signals extracted from the PMT's are cable summed $(\Sigma)$ and passed directly to a charge integrating ADC. 
FIG. 14. The typical NMM signal processing to generate start and stop signals for the Event Handler, and gate and analog signals for charge integrating ADC (QDC). (Legend: DS, downscaler; Delay, d_lay amplifier or digital delay generator; GG, gate generator)

FIG. 15. Timing diagram for signals associated with operating the Rochester NMM. (A) Event timing signal generated from a coincidence between "trigger" detector and NMM prompt response timing signals, identifying a reaction event. (B) NMM timing signals passed directly to the event handler "stop" input. (C) Delayed event timing signal directed to the event handler "start" input. (D) Counting gate the event handler generates internally when the start signal is present and the veto (E) is absent. (E) Event handler veto. Veto is set by the event handler start signal and removed with the CAMAC function CLEAR. (F) Gate triggered by the event handler start signal used to gate a QDC to record the NMM prompt response. (G) Logical AND of (B) and (D) which identifies NMM timing signals associated with the capture of a neutron by Gd. (H) Gate generated by the first signal (G) used to gate a QDC to record the NMM response to gamma rays emitted by the Gd nucleus excited by the capture of a neutron.

FIG. 16. Rochester NMM prompt response to $\gamma$-rays. The left panels include the background contribution to the light-output spectra, whereas in the right panels, the background contribution to the light-output spectra has been subtracted. Panels $\mathrm{A}$ and $\mathrm{B}$ are for a ${ }^{22} \mathrm{Na}$ source, panels $\mathrm{C}$ and $\mathrm{D}$ are for $\mathrm{a}^{60} \mathrm{Co}$ source, and panel $\mathrm{E}$ displays the background light-output spectrum.

FIG. 17. The light-output spectrum corresponding to the deexcitation of a ${ }^{156} \mathrm{Gd}$ or ${ }^{158} \mathrm{Gd}$ nucleus after capturing a neutron.

FIG. 18. Calibration of the Rochester NMM light-output signal to energy using various $\gamma$-ray sources and the Gd capture $\gamma$-rays.

FIG. 19. The Rochester NMM prompt light-output spectrum corresponding to the spontaneous fission of ${ }^{252} \mathrm{Cf}$. 


\section{TABLES}

\section{Table 1}

Gd-doped liquid scintillator BC-521

Compilation of chemical and physical parameters characterizing the liquid scintillator BC-521 for use in a neutron multiplicity meter

chemical composition

Base 1,2,4-trimethylbenzene

Gd-content $\quad 0.2 \%$ wt.

atomic densities $/ \mathrm{cm}^{-3}$

H

C

$\mathrm{O}$

Gd

$\mathbf{e}^{-}$
$5.231 \cdot 10^{22}$

$4.004 \cdot 10^{22}$

$3.409 \cdot 10^{20}$

$6.925 \cdot 10^{18}$

$2.958 \cdot 10^{23}$ physical parameters

Flashpoint

$44{ }^{\circ} \mathrm{C}$

$\alpha$

$0.555 \mu \mathrm{s}^{-1}$

$\beta$

$0.1085 \mu \mathrm{s}^{-1}$

density

$0.89 \mathrm{~g} / \mathrm{cm}^{-3}$

refractive index

1.50

decay time

$3.6 \mathrm{~ns}$

$\lambda_{\max }$

$424 \mathrm{~nm}$

bulk light attenuation $>4 \mathrm{~m}$

light output

$68 \%$ relative to

Anthrazene 


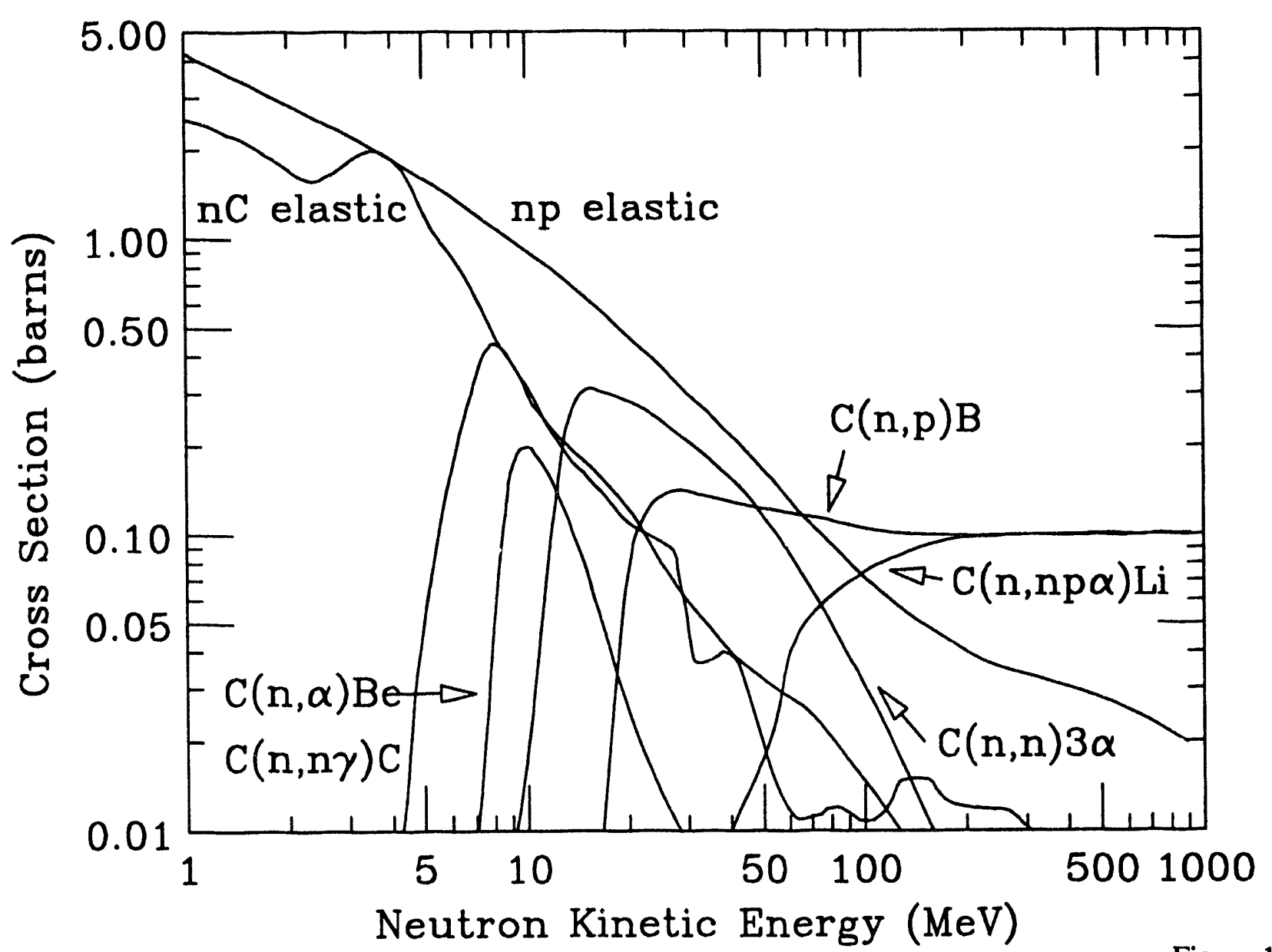

Figure 1

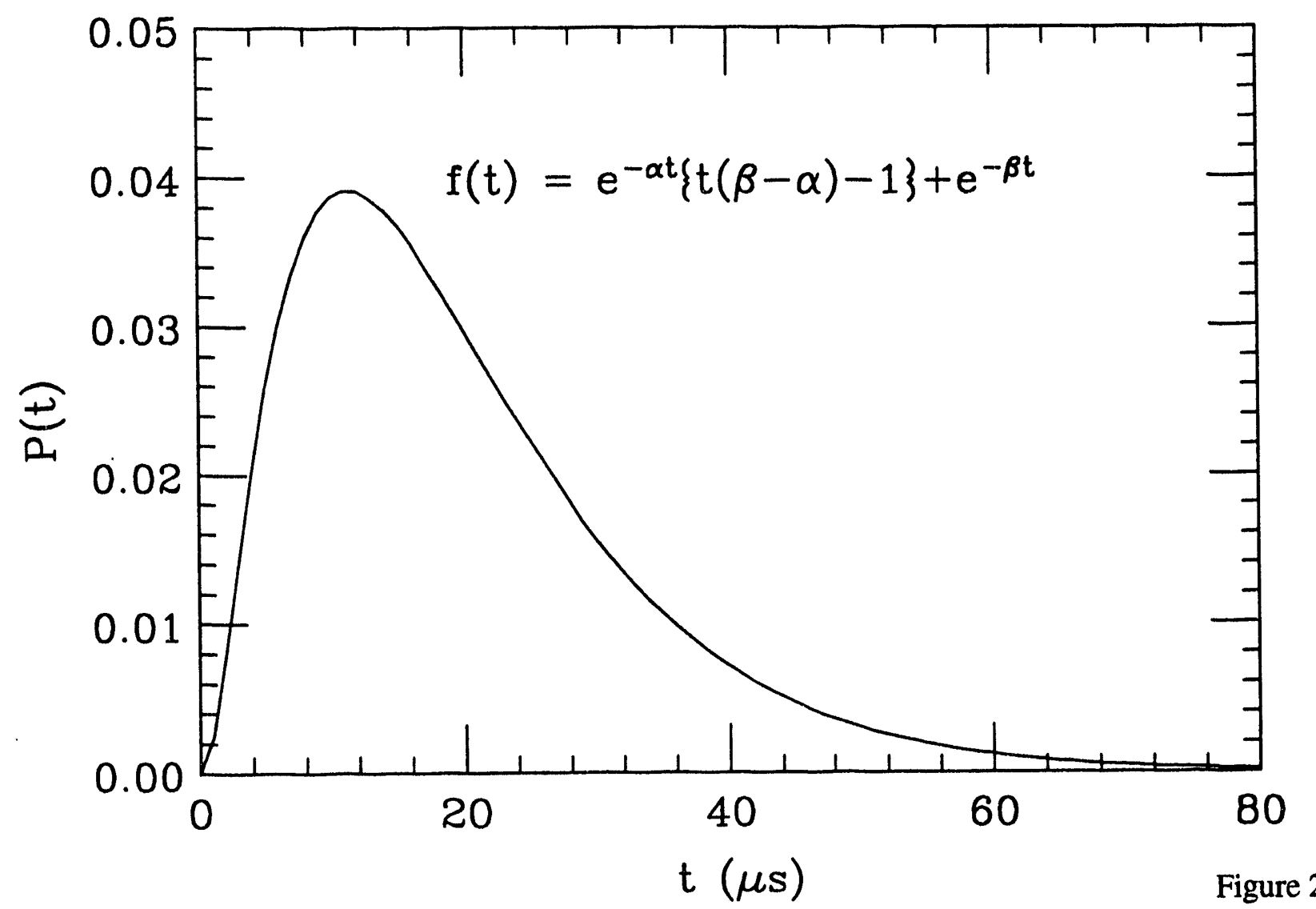





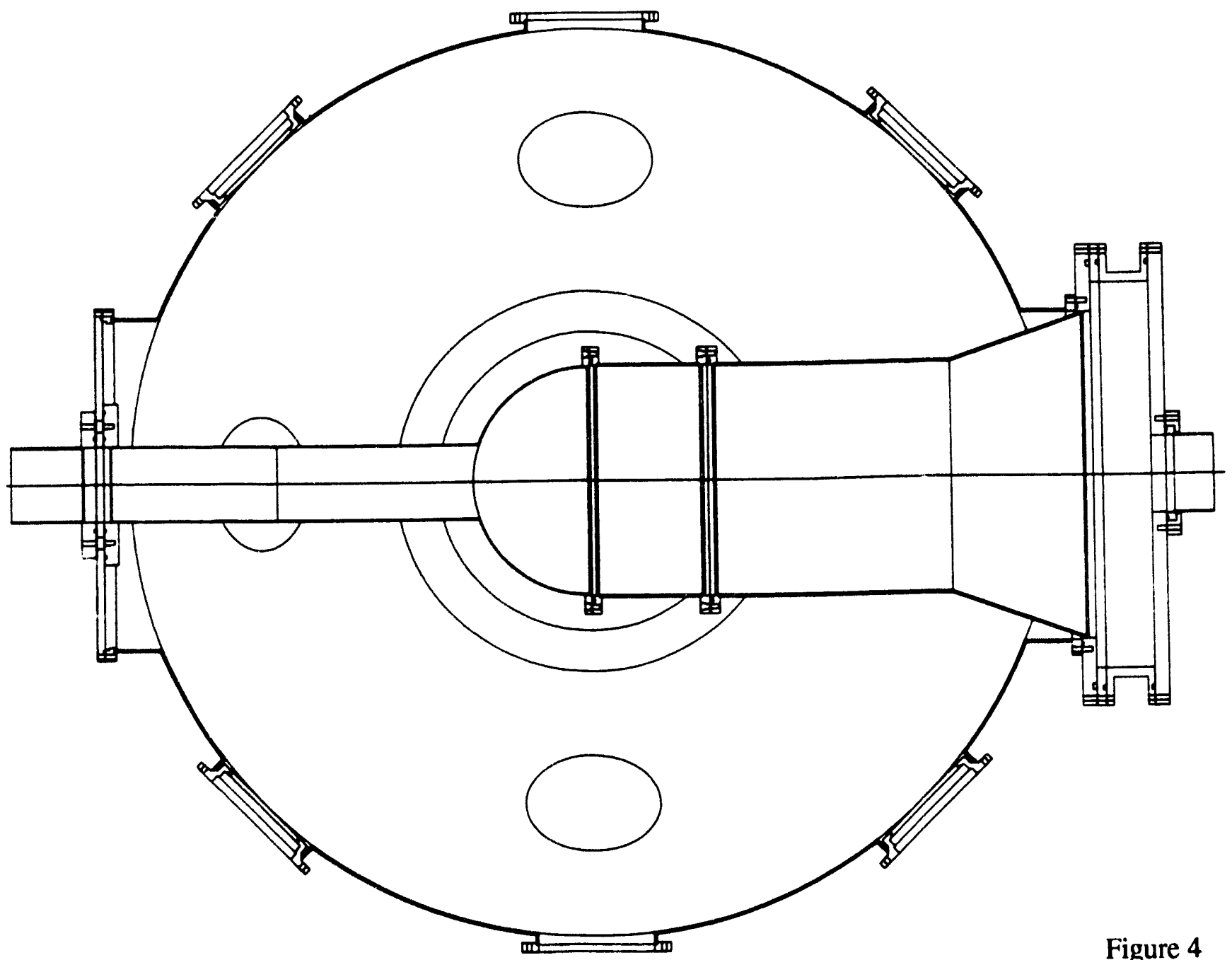

Figure 4

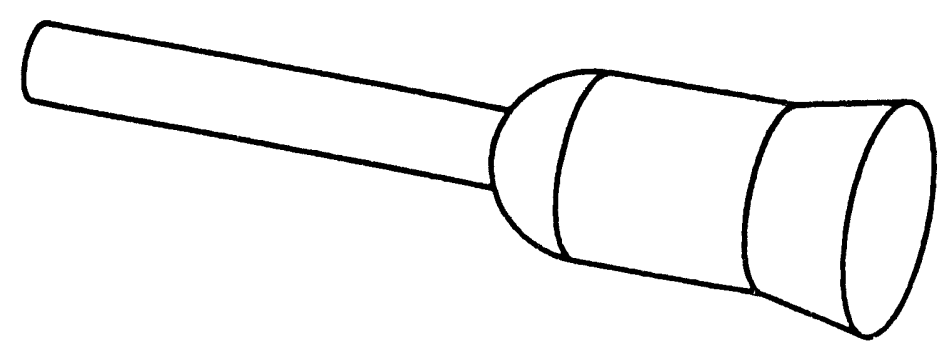

Figure 5 


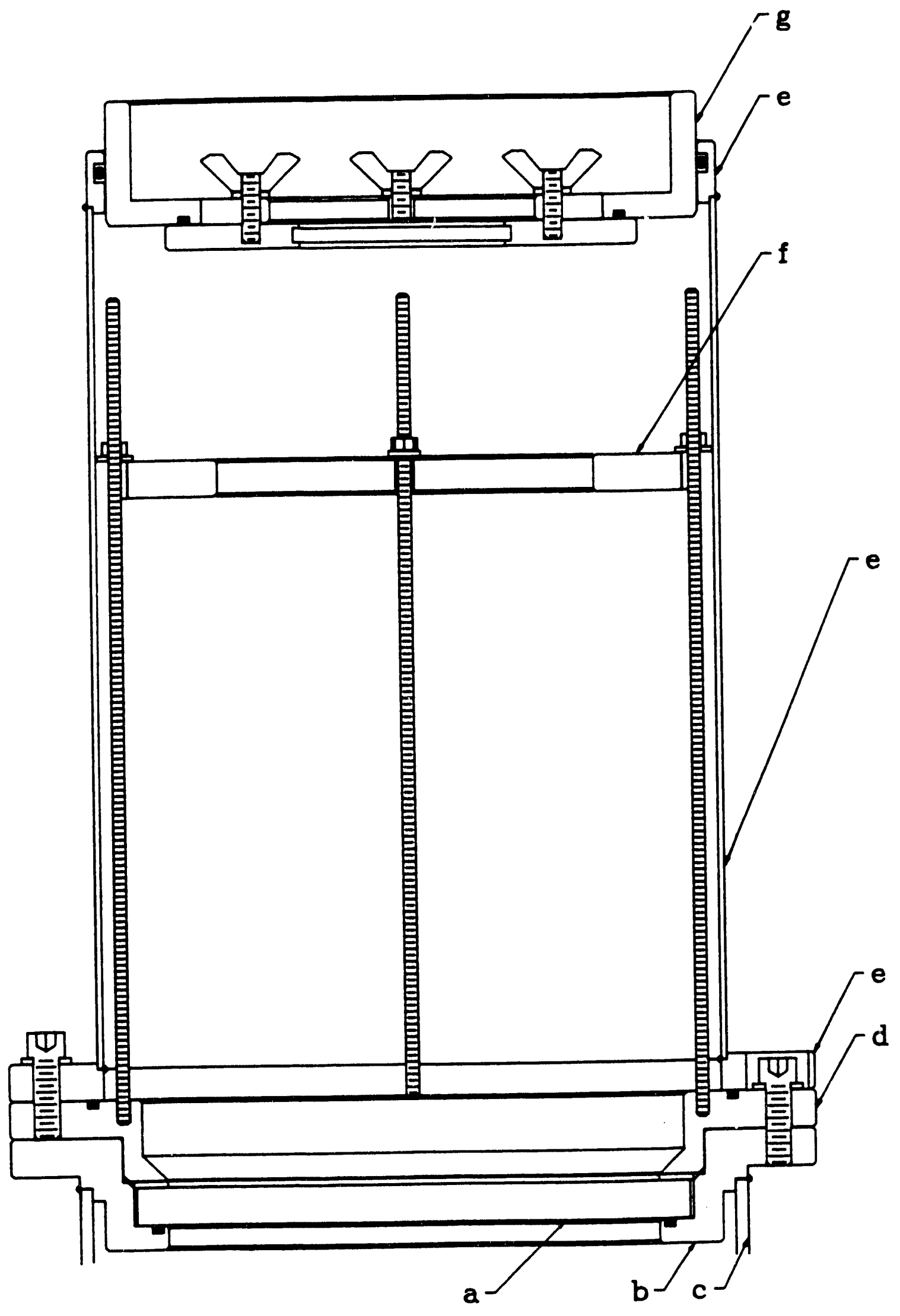

Figure 6 


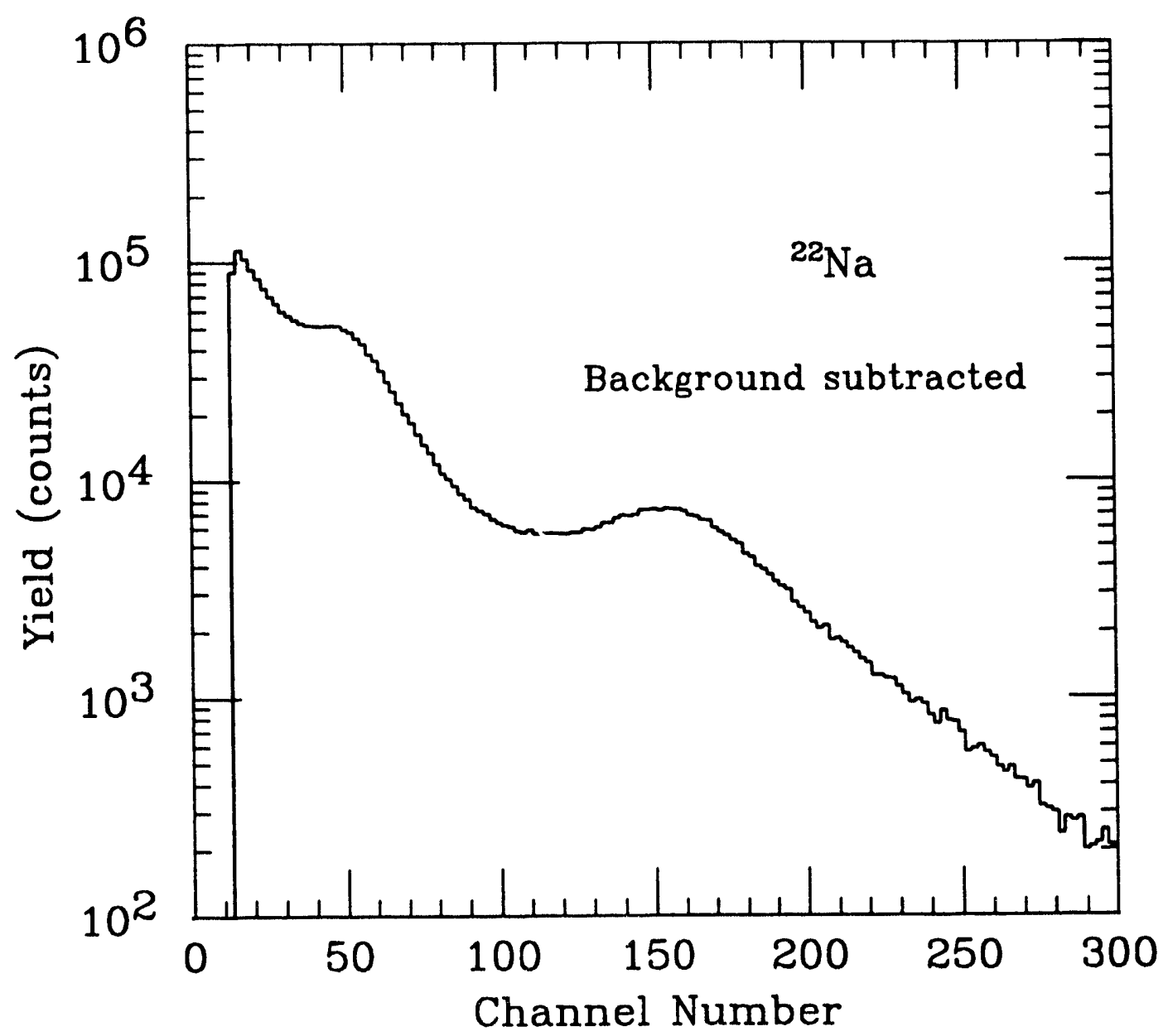

Figure 7 

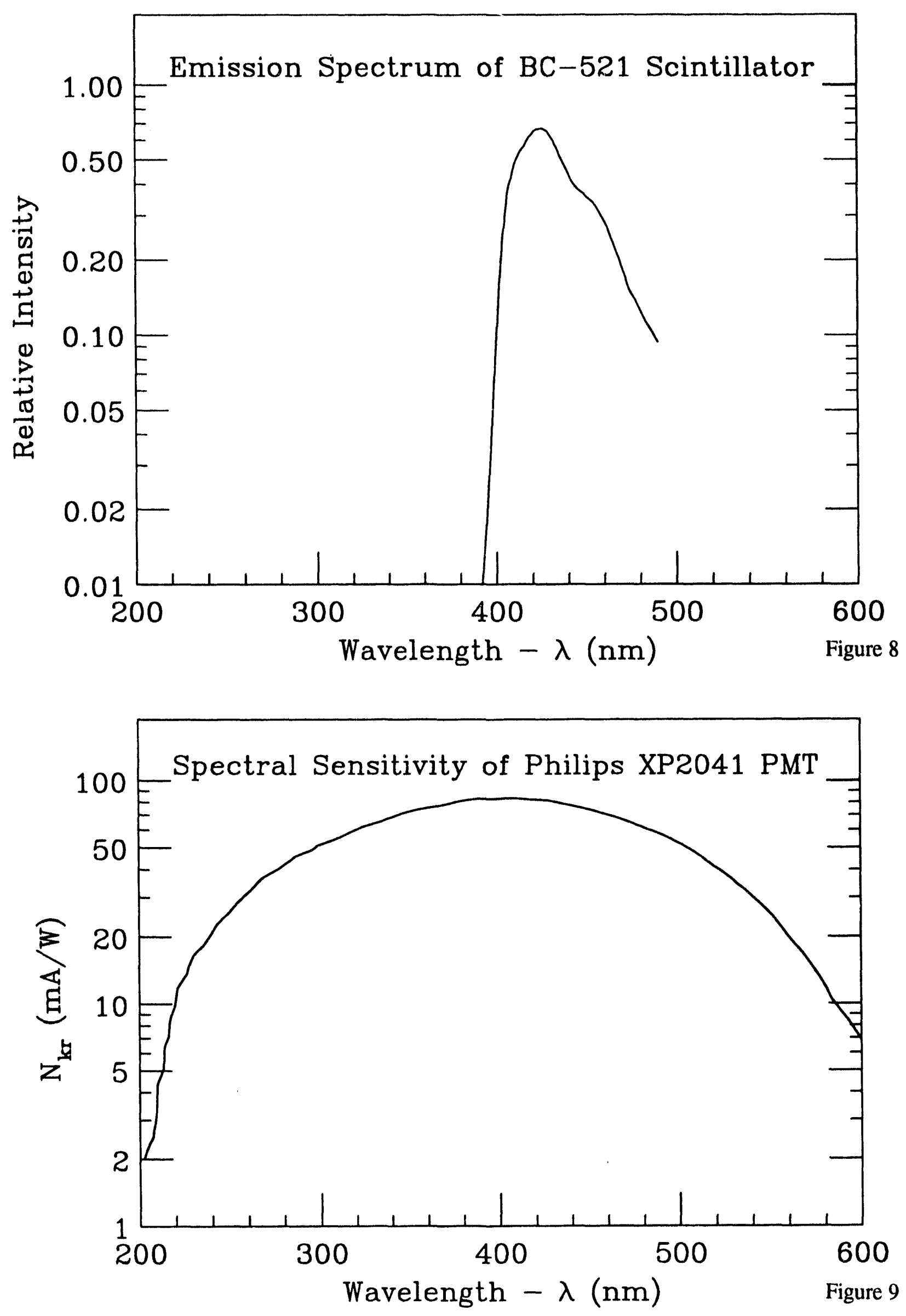


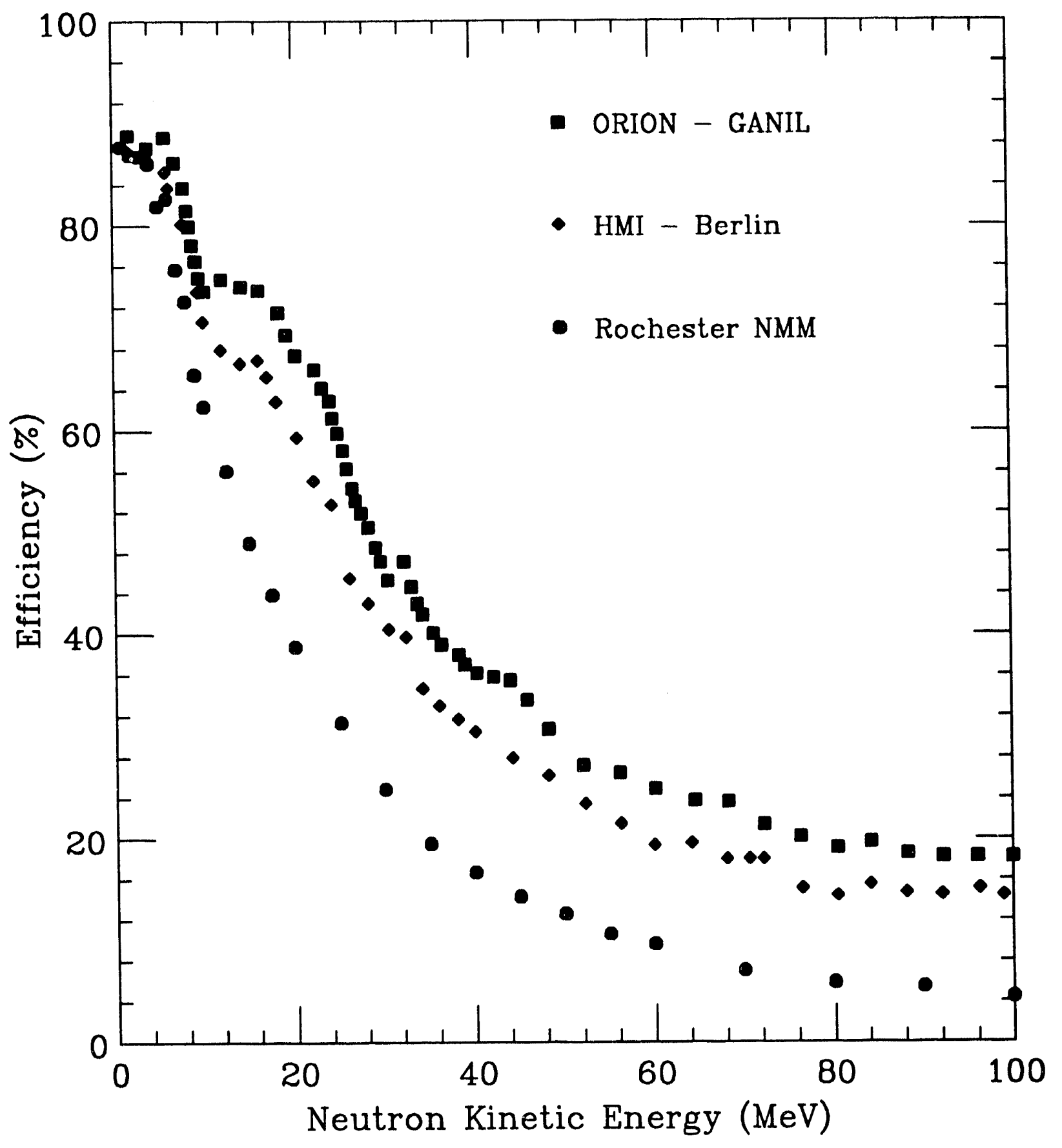

Figure 10 


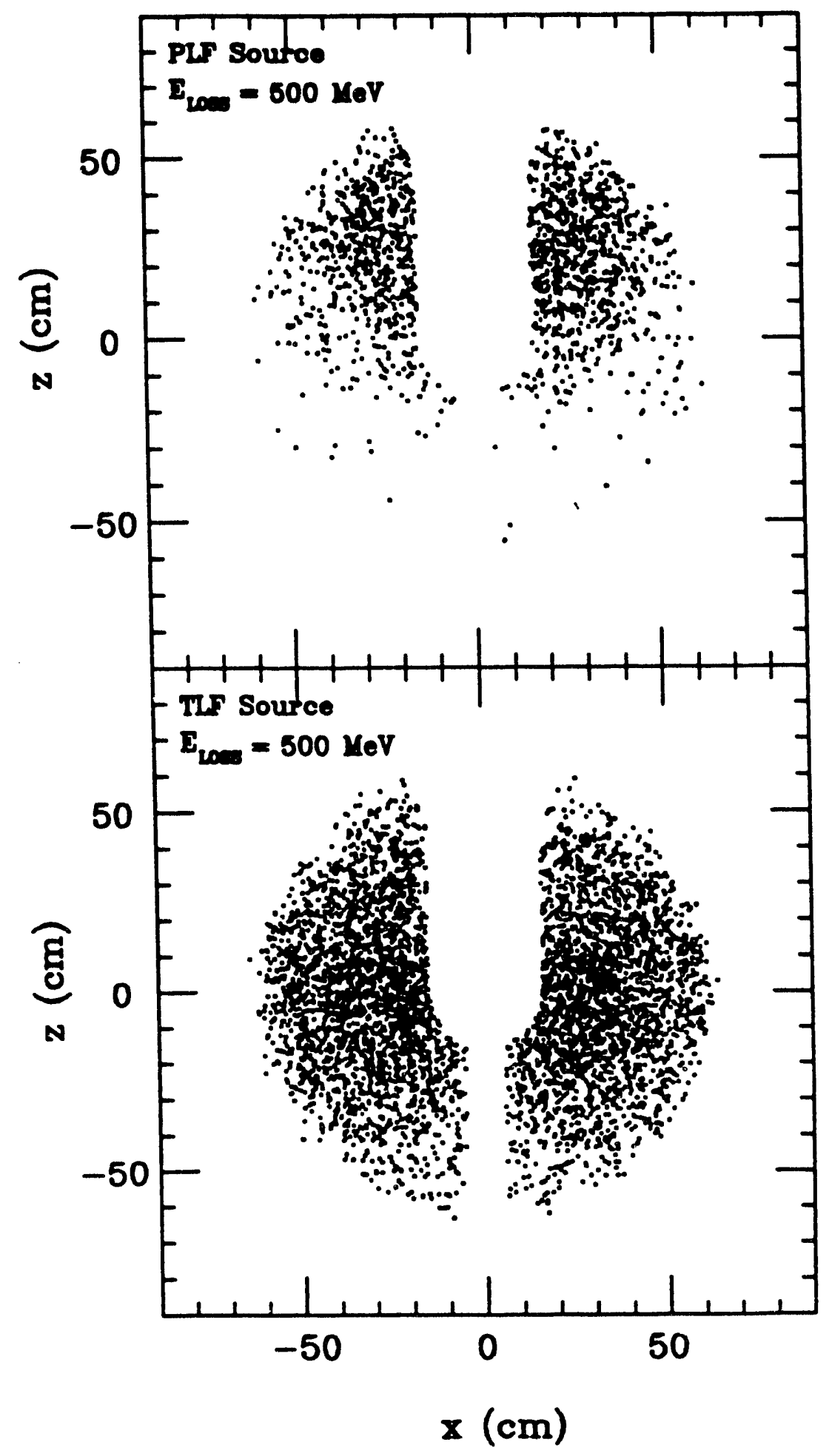

Figure 11 


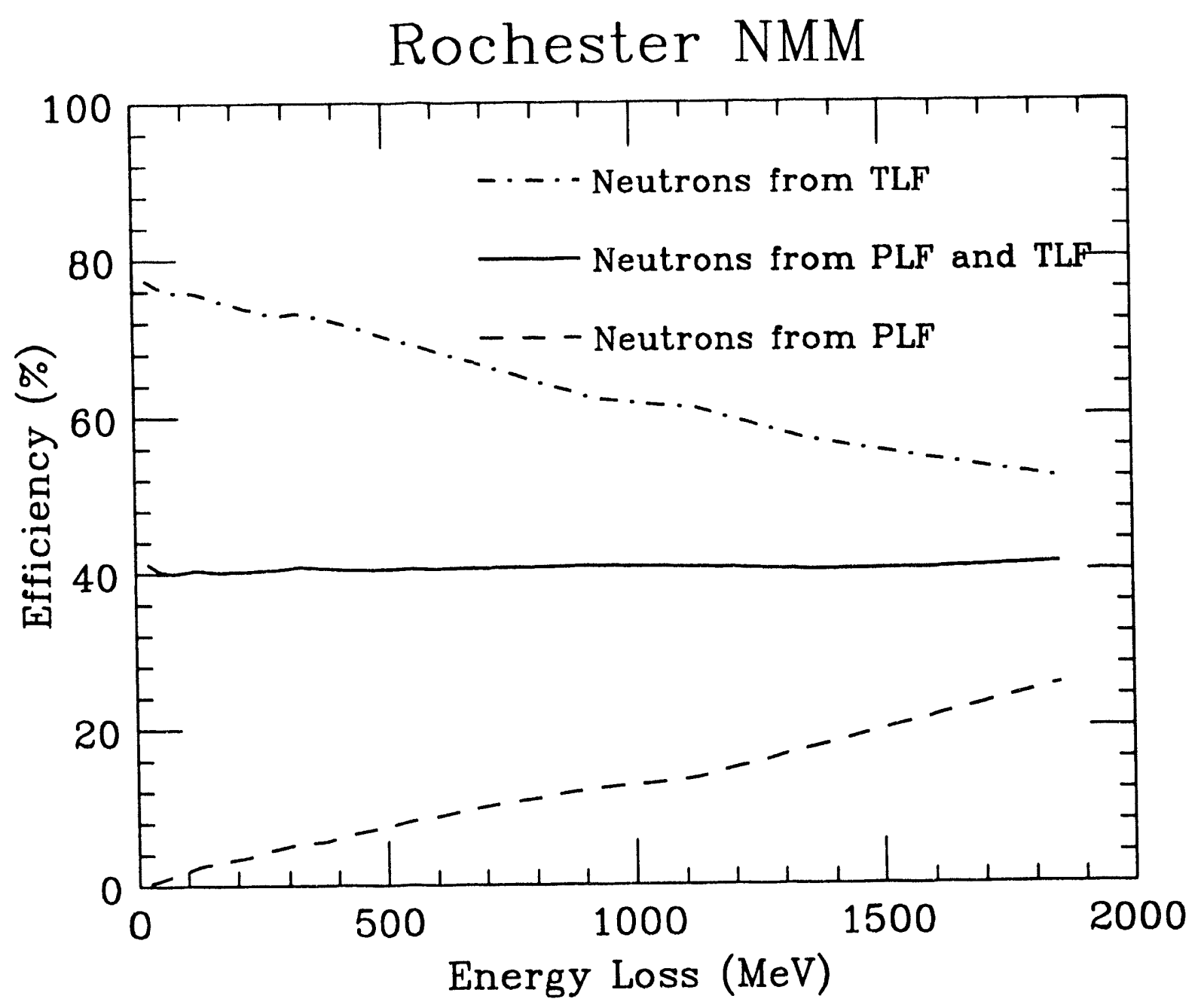

Figure 12 
Option A

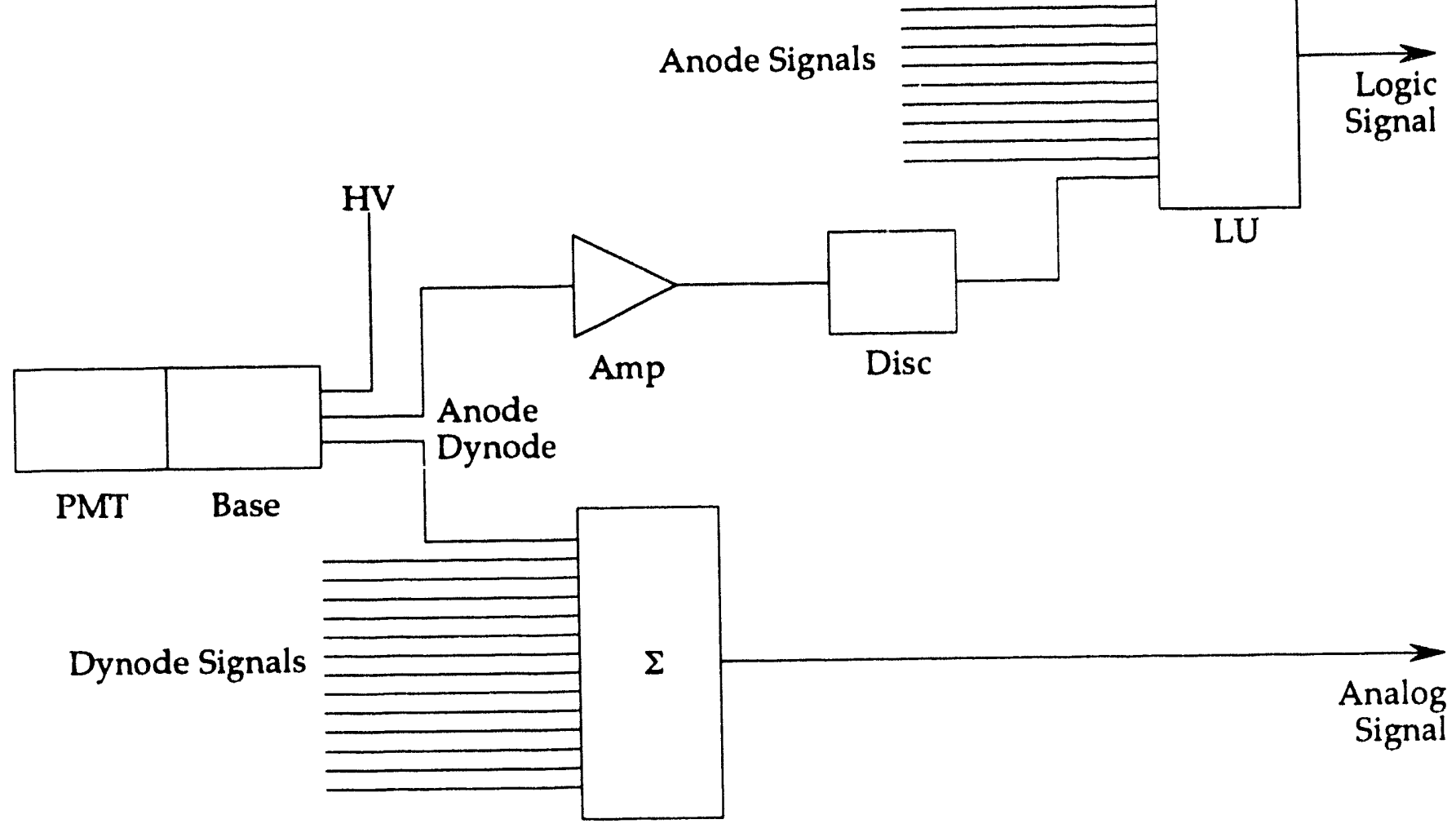

\section{Option B}

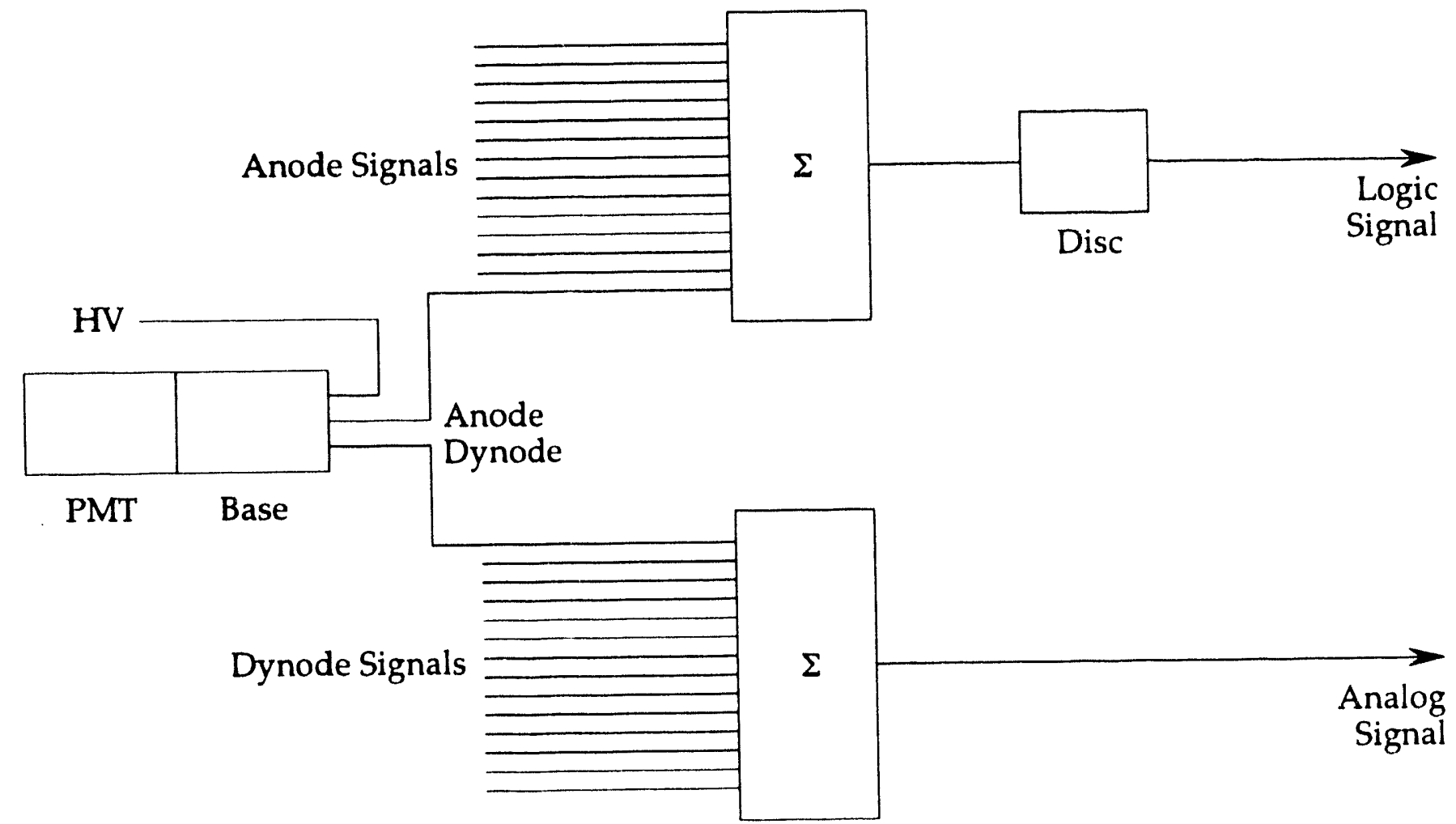

Figure 13 


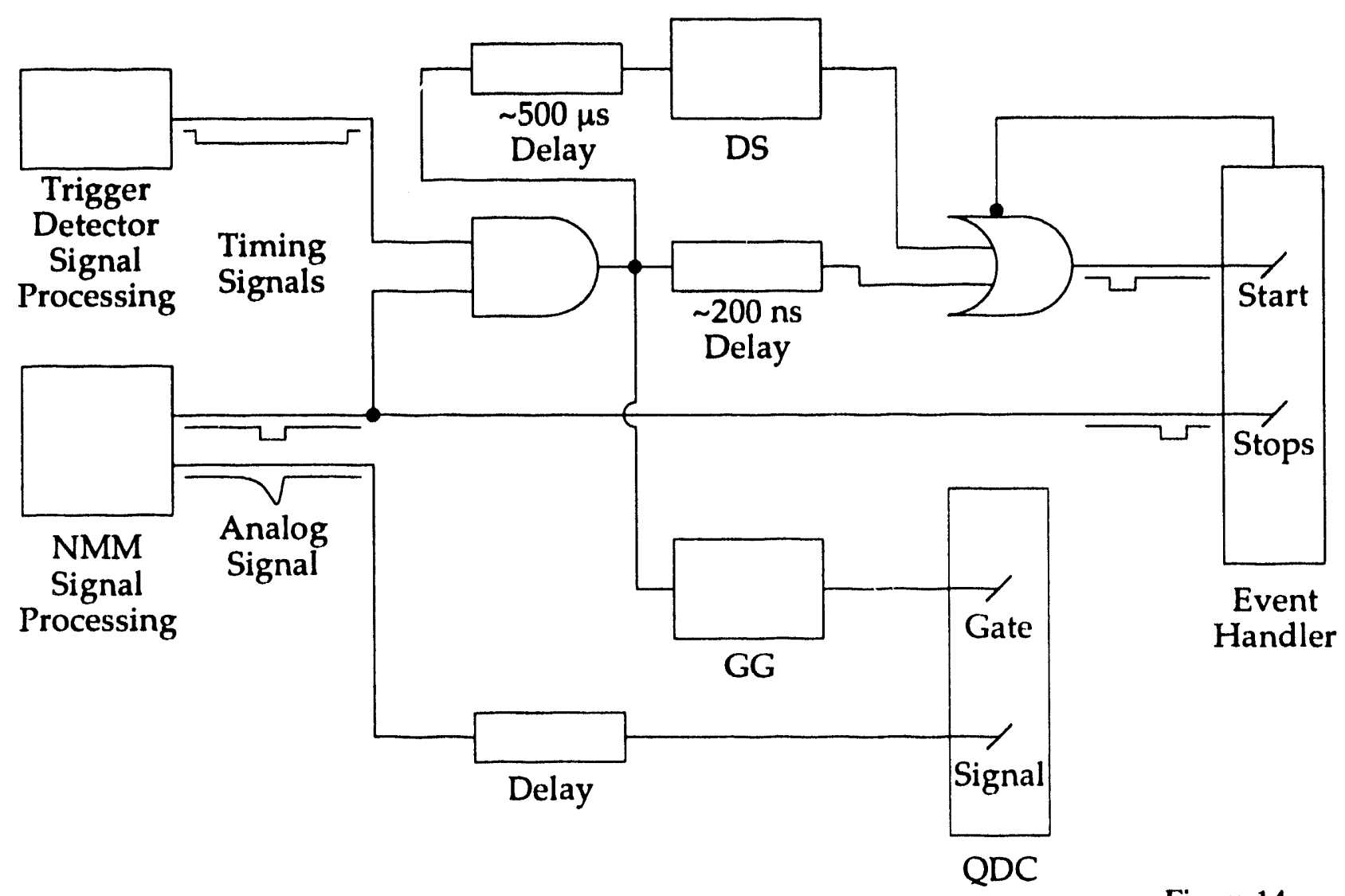

Figure 14

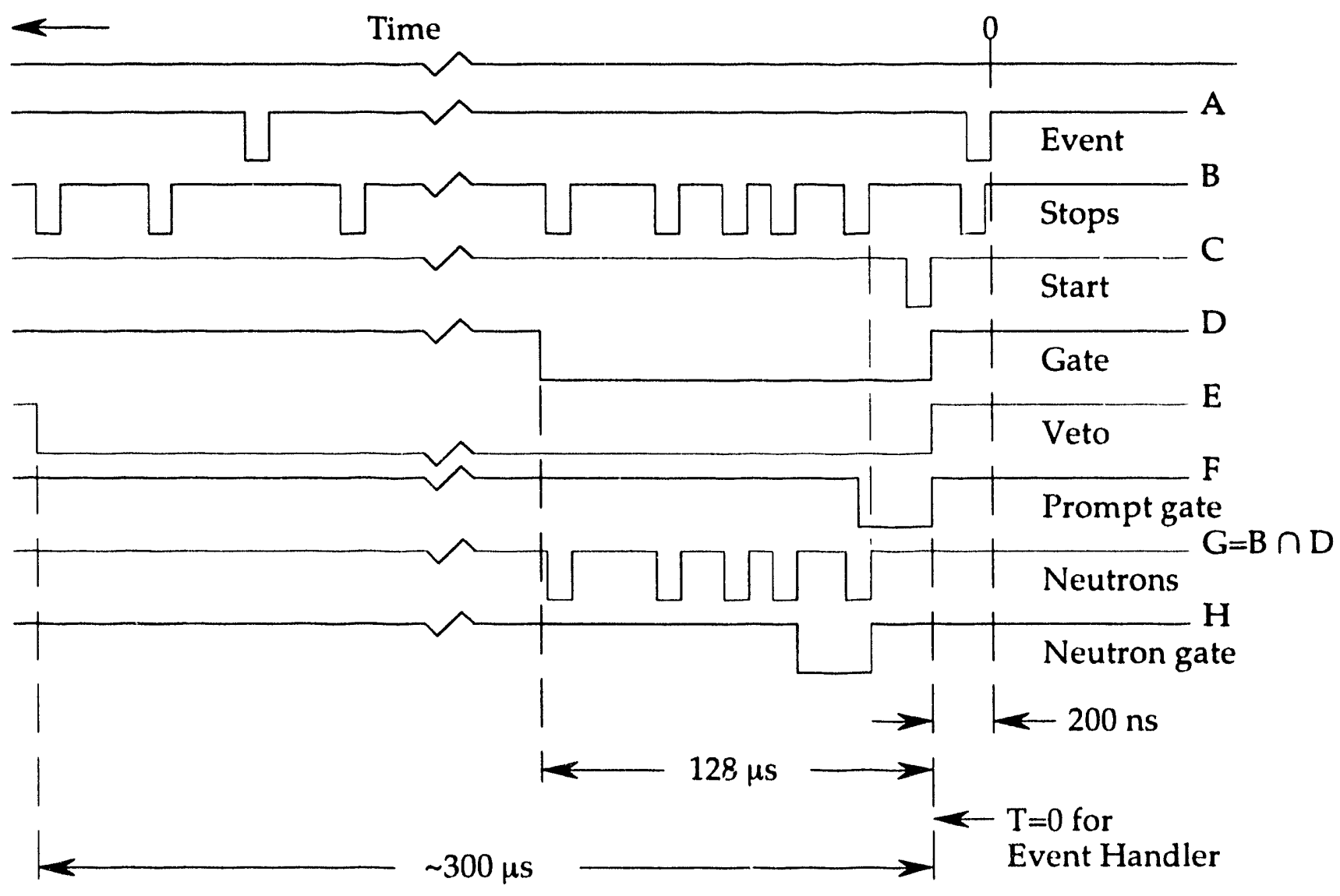

Figure 15 
Rochester NMM's Prompt Response

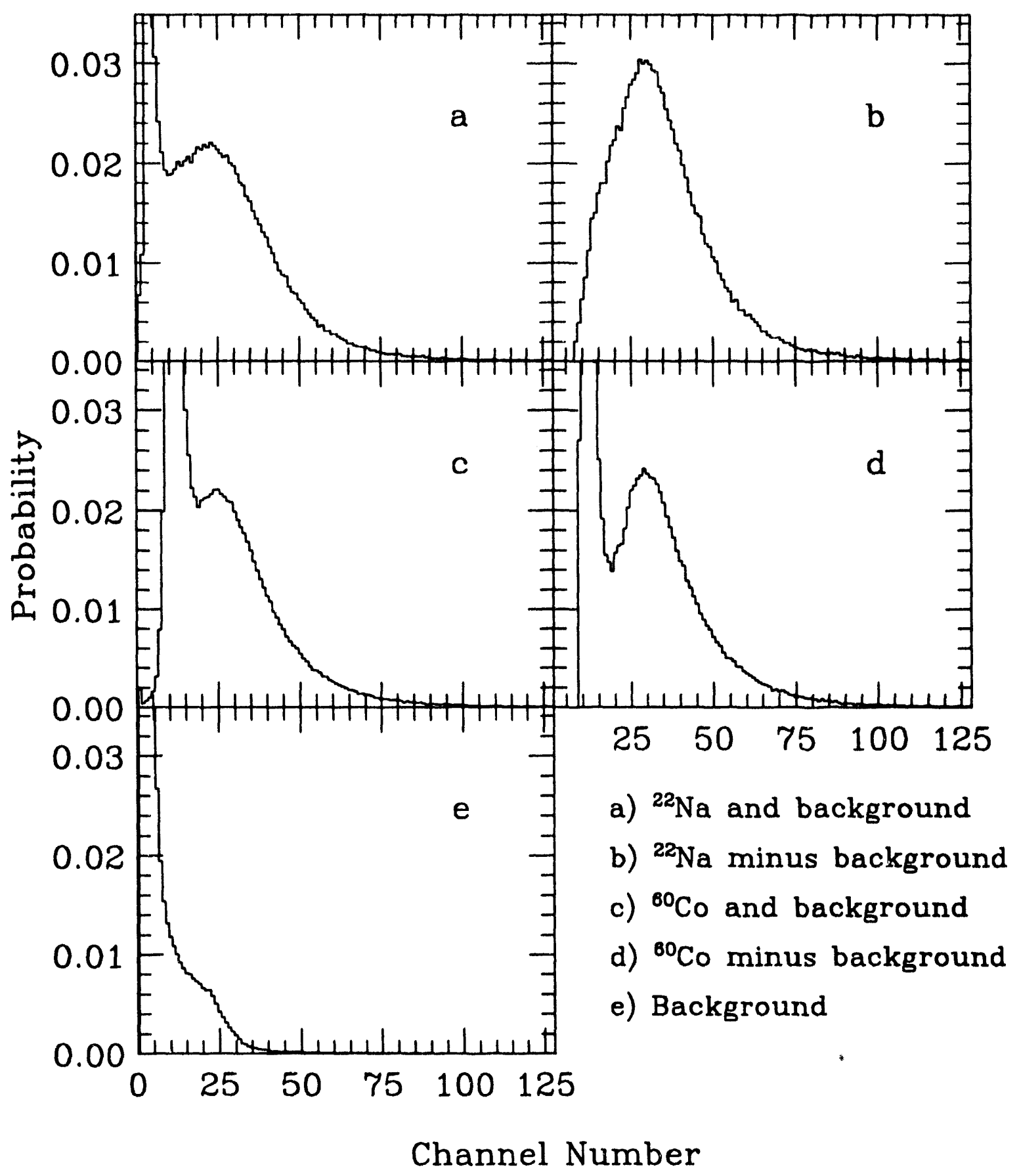

Figure 16 


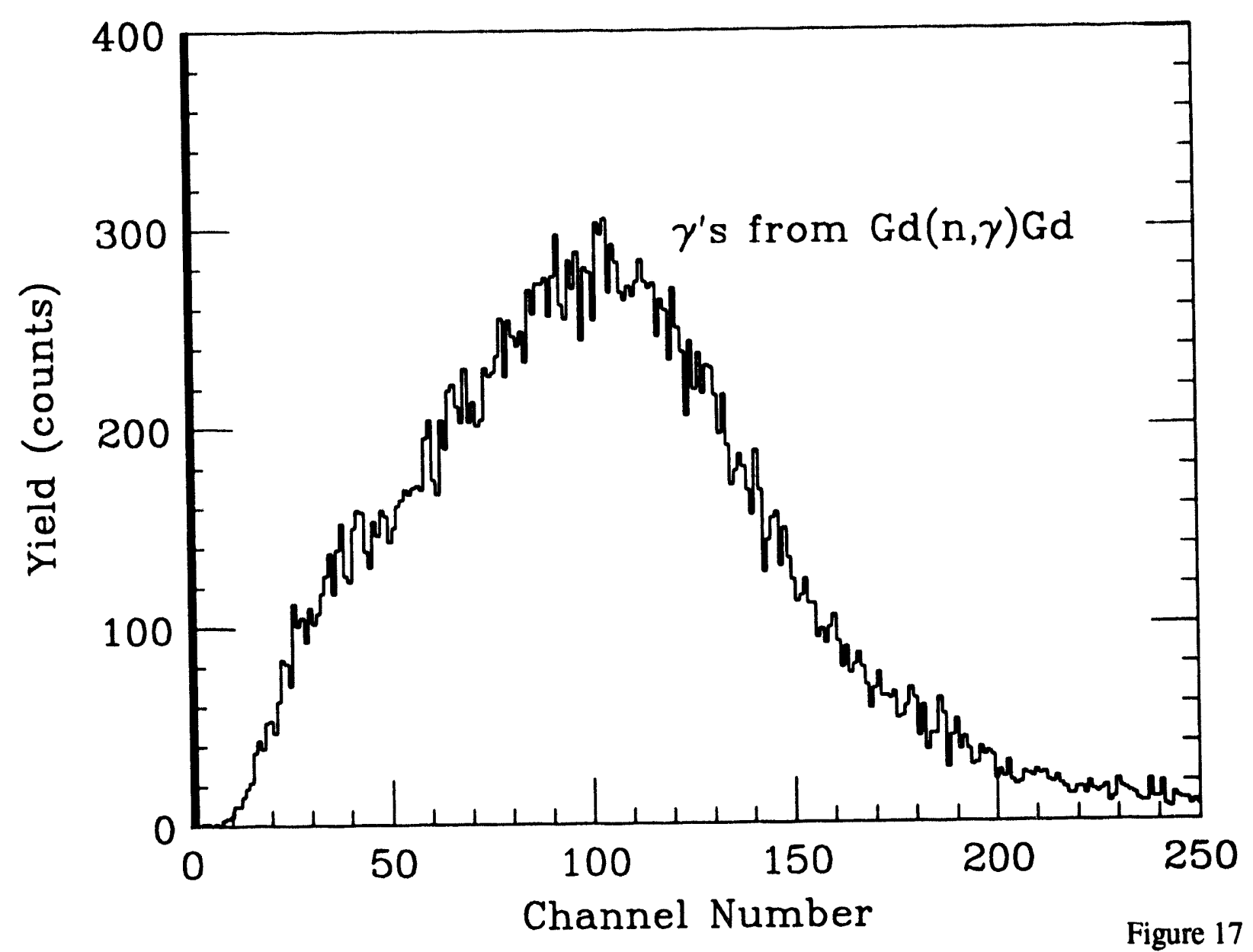

Figure 17

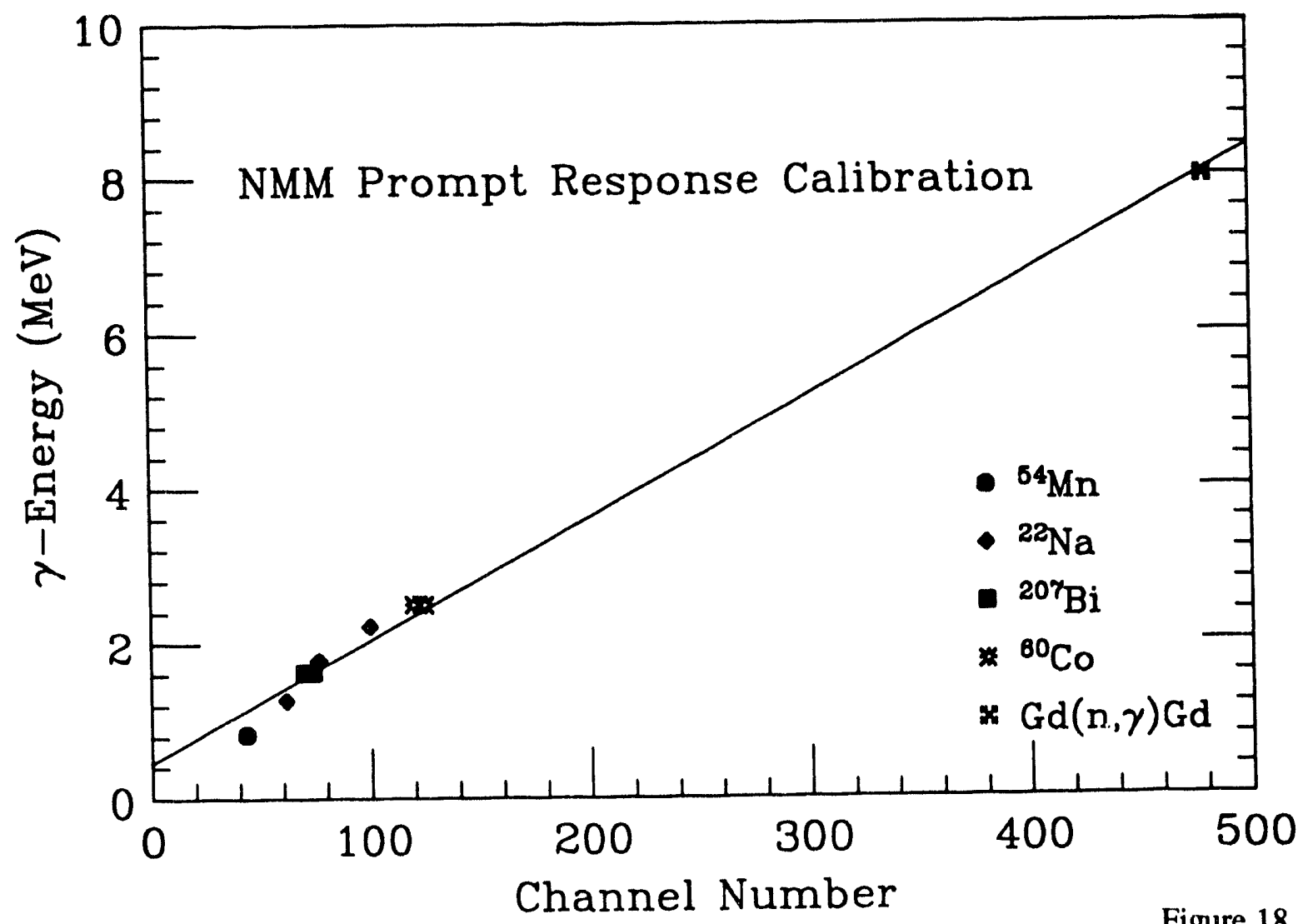

Figure 18 


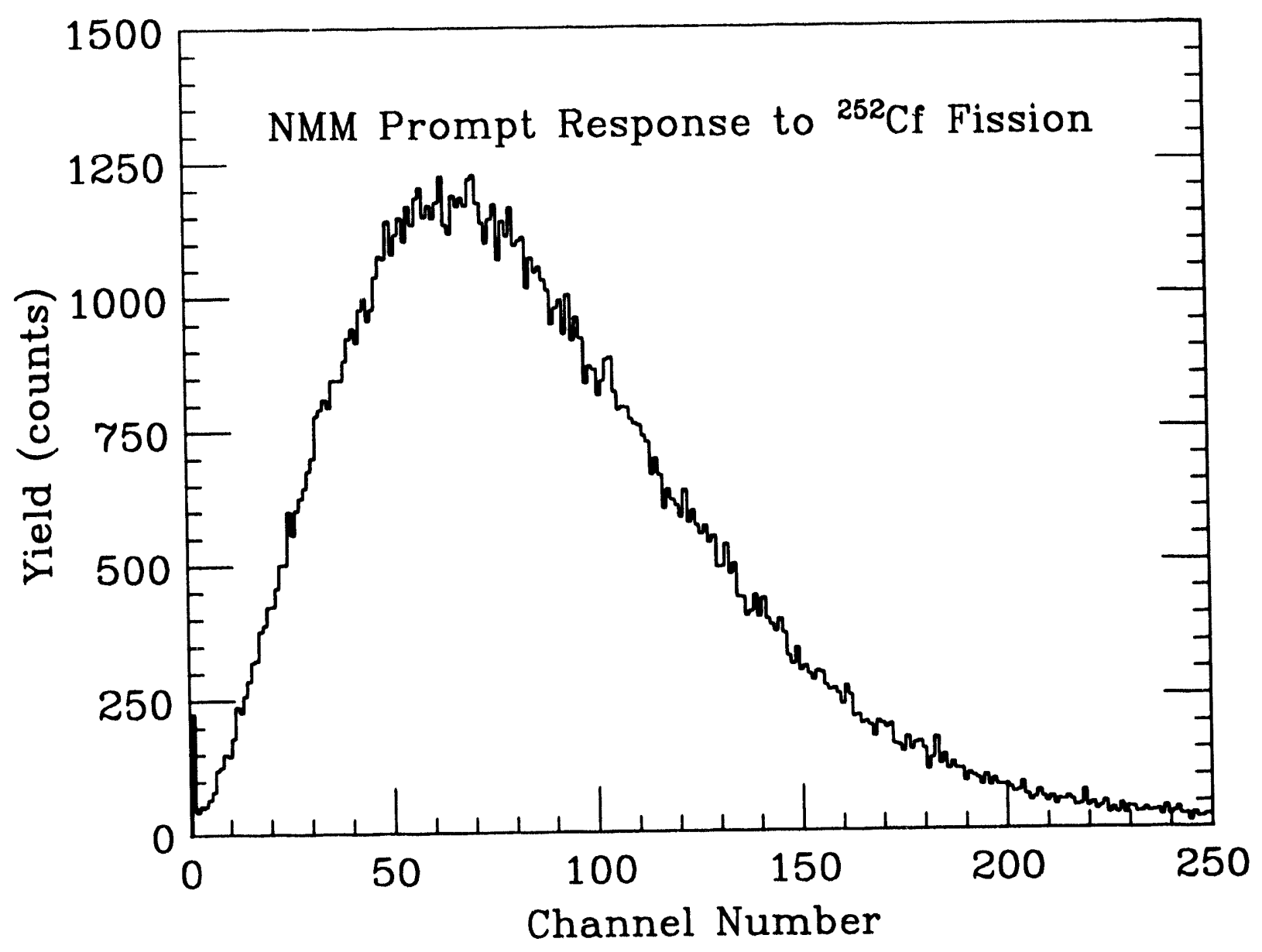

Figure 19 
A Simple Pulse Processing Concept for a Low-Cost Pulse-Shape-Based Particle Identification

\author{
J. Töke, S. A. Masserant, S. P. Baldwin, B. Lott, \\ W. U. Schröder, and X. Zhao \\ Department of Chemistry and Nuclear Structure Research Laboratory \\ University of Rochester, Rochester, iNY 14627, USA
}

\begin{abstract}
A simple method of pulse processing is described, which allows one to achieve at a very low cost a high-quality pulse-shape discrimination, using two commonly gated current-integrating ADCs. Results of application tests for a liquid NE213 scintillator detector (neutron- $\gamma$ discrimination), as well as for a $\mathrm{CsI}(\mathrm{Tl})$ detector ( $\mathrm{p}, \mathrm{d}, \mathrm{t}, \alpha$ discrimination) are presented.
\end{abstract}

It is a well-known fact that shapes of electronic pulses generated by nuclear radiation detectors depend noticeably on the species of the detected particles [1-6]. Various techniques have been employed to utilize this fact and to achieve particle identification through pulse-shape analysis. For cases where the speed of identification is critical, e.g., when rejection of an intense background of undesired radiation is the object, these techniques have been implemented in special pulse shape discriminator modules (PSD) such as the CANBERRA 2160 or the module PD6 developed by HMI Berlin. Such modules provide an electronic particle identification signal suited for fast electronic logics. However, these modules are relatively costly and may, therefore, be impractical in experiments involving large arrays of detectors. In these latter 
cases, a different approach appears to be more practical which relies on a digitization of certain portions of the detector signals and on off-line particle identification, made on software levels. Examples of such an approach are methods based on integration of the pulse current over two or more different time intervals, using current-integrating ADCs [1-6], or on a digitization of some pulse-shape information using TDCs or current-integrating ADCs [7]. Performing such a time-differential digitization with modular commercial electronics requires a series of well-timed gate signals. Alternatively, original detector signals may be processed using complex dedicated PSDs [7]. Either method causes major inconvenience in setting up and constitutes a costdriving factor in multi-detector experiments. In this letter, we report on a novel and very economical approach to pulse-shape discrimination. The method entails a very simple processing of primary detector signals to derive secondary signals which are then integrated over a liberally chosen common time interval, encompassing these signals entirely.

The method proposed in this work utilizes the fact that differentiation of a detector signal using an RC circuit yields a bipolar signal whose positive and negative parts, when plotted versus time, have equal areas. Both parts reflect the intensity and shape of only the leading part of the original signal, extending at most to the zero-crossing point. For example, in a purely mathematical differentiation, the absolute values of the time-integrals over the positive and negative parts of the differentiated signal are numerically equal to the peak-value of the original signal. In the method, either the positive or the negative part of the differentiated signal is rejected by hardware, while the remaining part is digitized with a current-integrating ADC. It appears that, exploiting the properties of a $\mathrm{p}-\mathrm{n}$ junction, it is easier to reject the short and high-amplitude first part of the differentiated signal than the lower and more extended under- or over-shoot signals. The method further utilizes a second current-integrating ADC to digitize the entire original signal. Clearly, both ADCs can use a common gate signal, since the only condition on the gate is that it encompasses both signals entirely. 
The value of the RC constant providing best pulse shape resolution is determined by the shape of the original pulses. In the tests reported here, no critical RC-dependence of the resolution was found, as long as the value of $\mathrm{RC}$ was comparable to the time constant of the fast component of the detector signal.

Fig. 1 shows a two-transistor implementation of the present particle identification method for pulses derived from the 14-th dynode of an AMPEREX XP2041 photomultiplier viewing the active volume of an NE213 liquid-scintillator neutron detector. The first stage of the circuit is built around a high-frequency npn-type transistor (T1), operated at a quiescent collector current of approximately $1 \mathrm{~mA}$. This stage yields an inverted "collector image" and a non-inverted "emitter image" of the original signal. The emitter signal is differentiated with a time constant of approximately $45 \mathrm{~ns}$ determined by $\mathrm{C} 1$ and $\mathrm{R} 1$, and processed further, using the fast pnp-type transistor T2. This latter transistor is biased so as to ensure rejection of the first, positive part of the differentiated signal. In the tests reported here, was adjusted to allow for a quiescent collector current of $50 \mu \mathrm{A}$.

Results of tests with an NE213 liquid-scintillator detector and a ${ }^{252} \mathrm{Cf}$ neutron- $\gamma$ source placed in front of it, are illustrated in Figs. 2 and 3. Fig. 2 shows signals from the collector of $\mathrm{T} 1$ (full signal, top) and emitter of T2 (undershoot, bottom), as observed on the CRT screen of a fast oscilloscope. These two signals were subsequently digitized, using a LeCroy $2249 \mathrm{~W}$ current-integrating CAMAC ADC module to yield values of total charge, $Q_{t o t}$, and the charge carried by the leading portion of the detector signal, $Q_{\text {fast }}$, respectively. A 300-ns long ADC gate signal was used, derived from the original signal. To demonstrate that timing of this gate pulse is not critical for the quality of pulse shape discrimination obtained with the present method, the gate was purposely set so as to encompass not only the main pulses but also their small negative and positive parasitic precursors.

Fig. 3 displays a scatter plot of $Q_{\text {fast }}$ versus $Q_{t o t}$, as acquired with the above NE213 detector setup. An excellent separation of the neutron and $\gamma$ branches is 
seen, for a range of energies starting from approximately $100 \mathrm{keV}$ of equivalent electron energy. It has been verified in a further test that, by using the photomultiplier anode signal and a processing circuit properly modified for the pulse polarity, the discrimination threshold can readily be lowered to $50 \mathrm{keV}$ of equivalent electron energy. Fig. 4 shows a spectrum of the particle identification pseudo-parameter (PID), defined through a linearization of the branches seen in Fig. 3. Again, an excellent separation of events due to neutrons and $\gamma$ quanta is seen.

Fig. 5 shows results of the tests in which a $\mathrm{CsI}(\mathrm{Tl})$ crystal, viewed by HAMAMATSU R1666HA photomultiplier, was used to detect products of the ${ }^{27} \mathrm{Al}+(100$ $\mathrm{MeV})^{16} \mathrm{O}$ reaction. In this case, a commercial ORTEC 474 timing-filter amplifier was employed to invert, amplify, and differentiate the anode signal, with a timing constant of 500 ns. The first, positive part of the differentiated signal was then rejected using a fast pnp-type transistor, operated in a regime similar to that of T2 shown in Fig. 1 (quiescent collector current of $50 \mu \mathrm{A}$ ). Again, the original signal and the undershoot of the differentiated signal were current-integrated with a LeCroy 2249W ADC to yield values of $Q_{t o t}$ and $Q_{f a s t}$, respectively. In this case, the length of the ADC gate was set to $2 \mu \mathrm{s}$. An excellent resolution of the branches corresponding to protons, deuterons, and $\alpha$-particles is seen in Fig. 5, starting from energies of only a few $\mathrm{MeV}$.

Similar tests were carried out for a phoswich detector composed of a $0.2-\mathrm{mm}$ thick, fast NE102 plastic scintillator backed by a thicker NE115 plastic, as well as for a silicon detector. In all cases, the quality of the particle ID proved comparable to that achieved using the "standard" technique of current-integrating the signal $[1,2,6]$ over two different time intervals (correlated gates).

A simple generalization of the method is possible for those detector types, whose light output has more than two components, e.g., like those utilized in the Washington University DWARF array [3,4] or the MSU MINIBALL array [5]. The detector elements of the latter arrays are composed of a thin fast plastic, backed by a thick CsI(Tl) crystal. These detectors, hence, generate signals with three distinct com- 
ponents. The generalization of the method presented in this work on such a case would entail generation of two undershoot signals, using two different differentiation time constants. Again, a single ADC gate signal, generated from the reaction master trigger signal, can be used in a reproducible fashion to digitize information on the total light output, as well as on two different components of the detector signal.

In summary, the proposed simple concept allows one to achieve, at very little effort and at a very low cost, pulse-shape discrimination with a quality equal to that provided by the "standard" electronically more involved methods. The simplicity, low cost, and inherent reproducibility make the proposed method a natural choice for large multi-hit arrays of detectors, such as multi-element NE213 neutron spectrometers, CsI(Tl) and phoswitch detector arrays.

This work was supported by the U.S. Department of Energy under grant number DE-FG02-88ER40414.

\section{References}

[1] F. Lidén, J. Nyberg, A. Johnson and A. Kerek, Nucl. Instr. and Meth. A253 (1987) 305.

[2] J. Pouliot, et al., Nucl. Instr. and Meth. A270 (1988) 69.

[3] D.G. Sarantites, et al., Nucl. Instr. and Meth. A264 (1988) 319.

[4] D.W. Stracener, et al., Nucl. Instr. and Meth. A294 (1990) 485.

[5] R.T. De Souza, et al., Nucl. Instr. and Meth. A295 (1990) 109.

[6] G. Pausch, et al., Preprint HMI-P/92/P2-Pau1, Berlin, 1992.

[7] J. Kasagi, T. Murakami and T. Inamura, Nucl. Instr. and Meth. A236 (1985) 426 . 


\section{Figure Captions}

Fig. 1. Electronic circuit diagram for processing of positive-polarity detector signals.

Fig. 2. Samples of primary NE213 detector signals (top) and of corresponding secondary, "undershoot" signals (bottom)

Fig. 3. Scatter plot of the charge, $Q_{f a s t}$, of the fast component versus total charge, $Q_{t o t}$, for signals generated by neutrons or $\gamma$-rays, respectively, in an NE213 scintillator detector.

Fig. 4. Spectrum of particle identification pseudoparameter, PID, for the data of Fig. 3.

Fig. 5. Same as Fig. 3, except for a $\mathrm{CsI}(\mathrm{Tl})$ detector. 


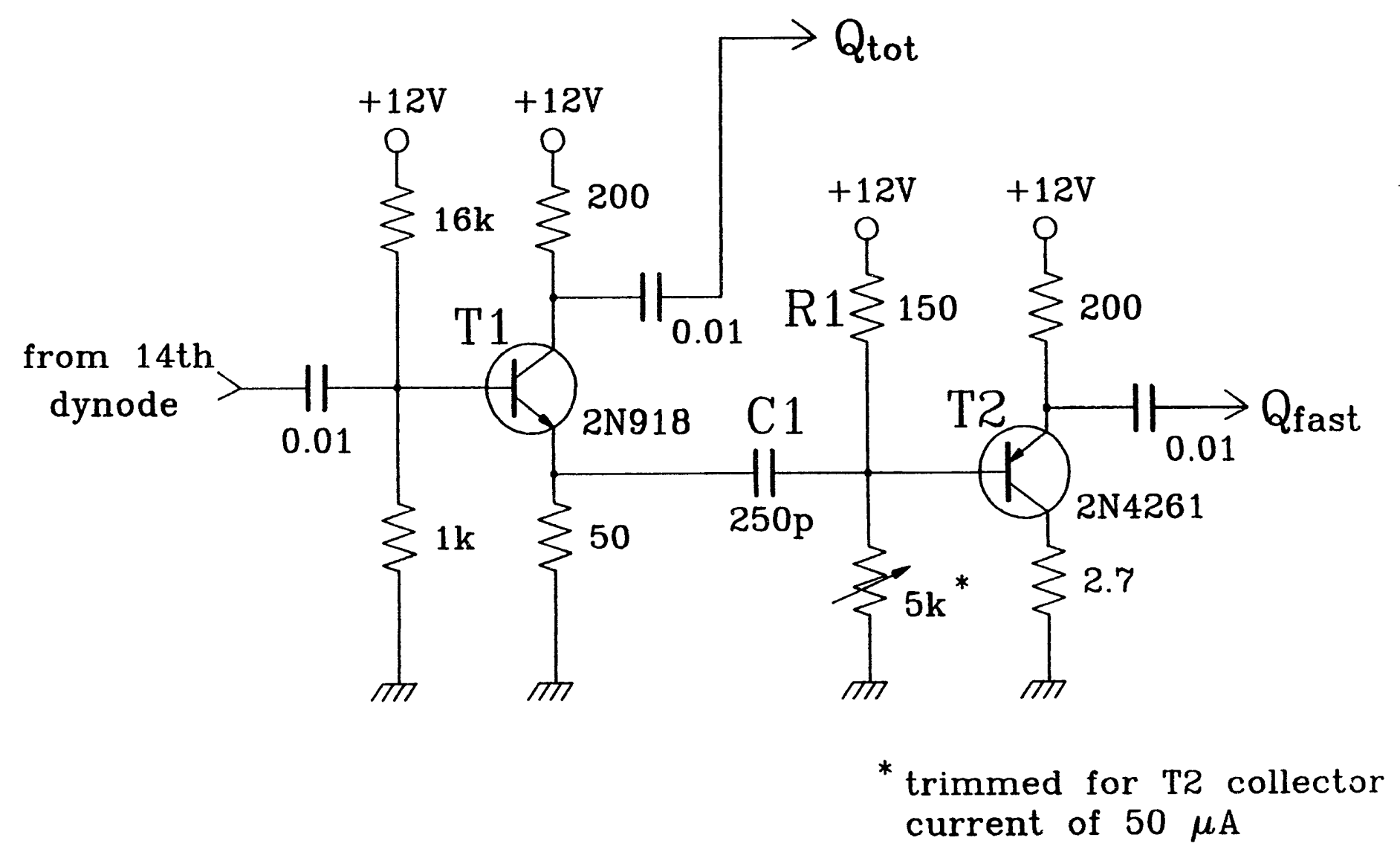




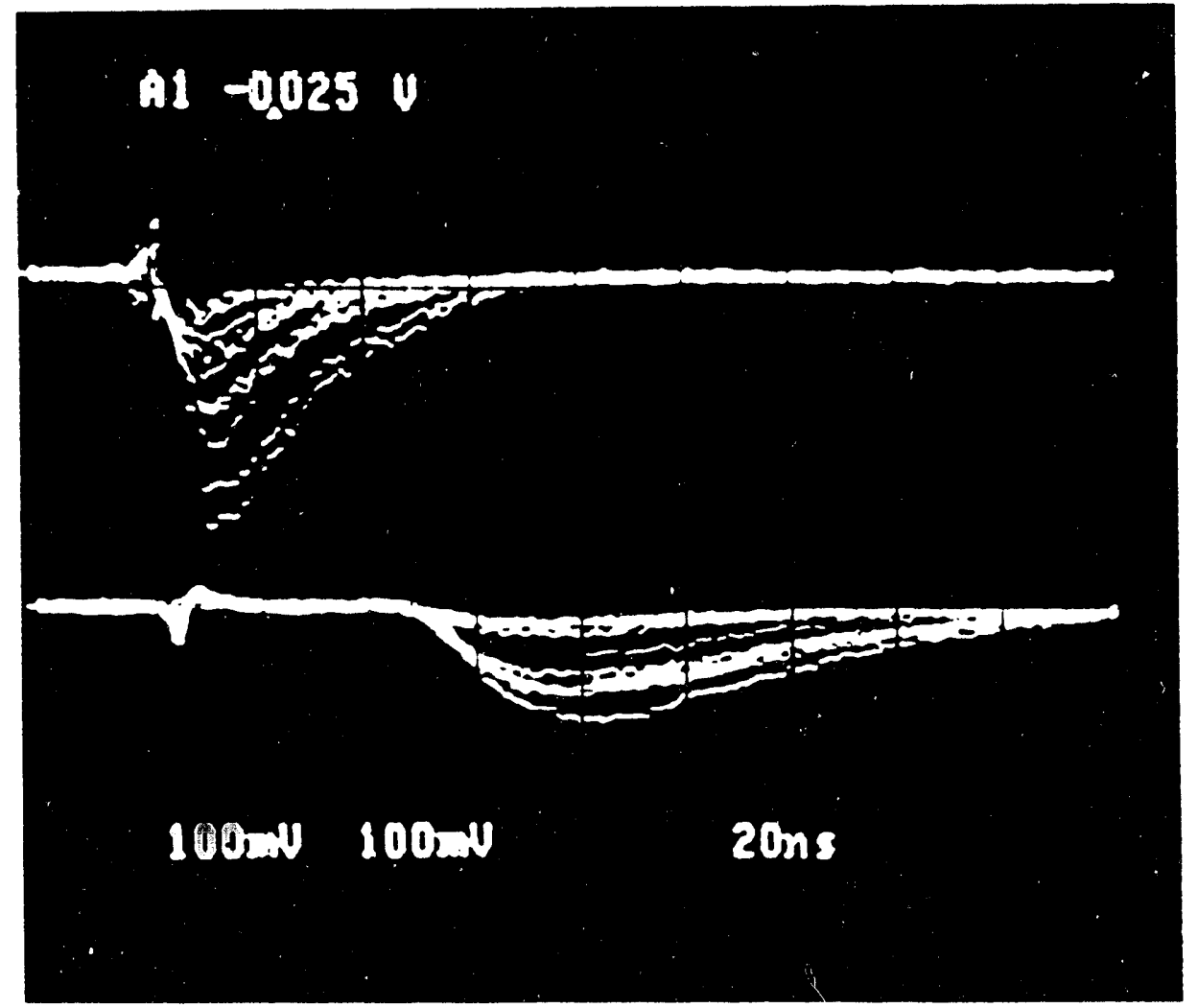

Fig. 2: Samples of primary NE213 detector signals (top) and of corresponding secondary, "undershoot" signals (bottom). 


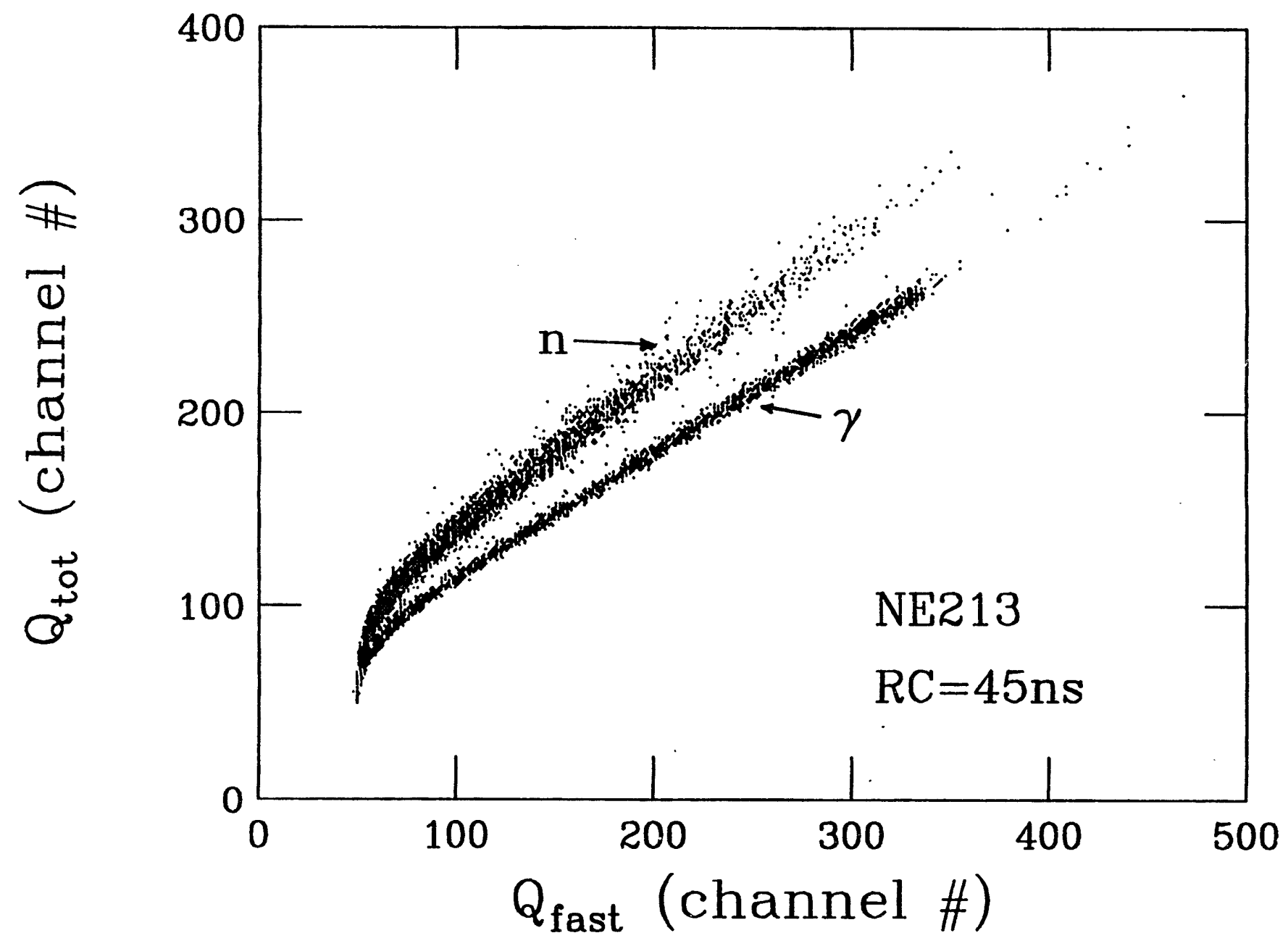

Fig. 3: Scatter plot of the charge, $Q_{f a c t}$, of the fast component versus total c'sarge, $Q_{t o t}$, for signals generated by neutrons or $\gamma$-rays, respectively, in an NE213 scintillator detector. 


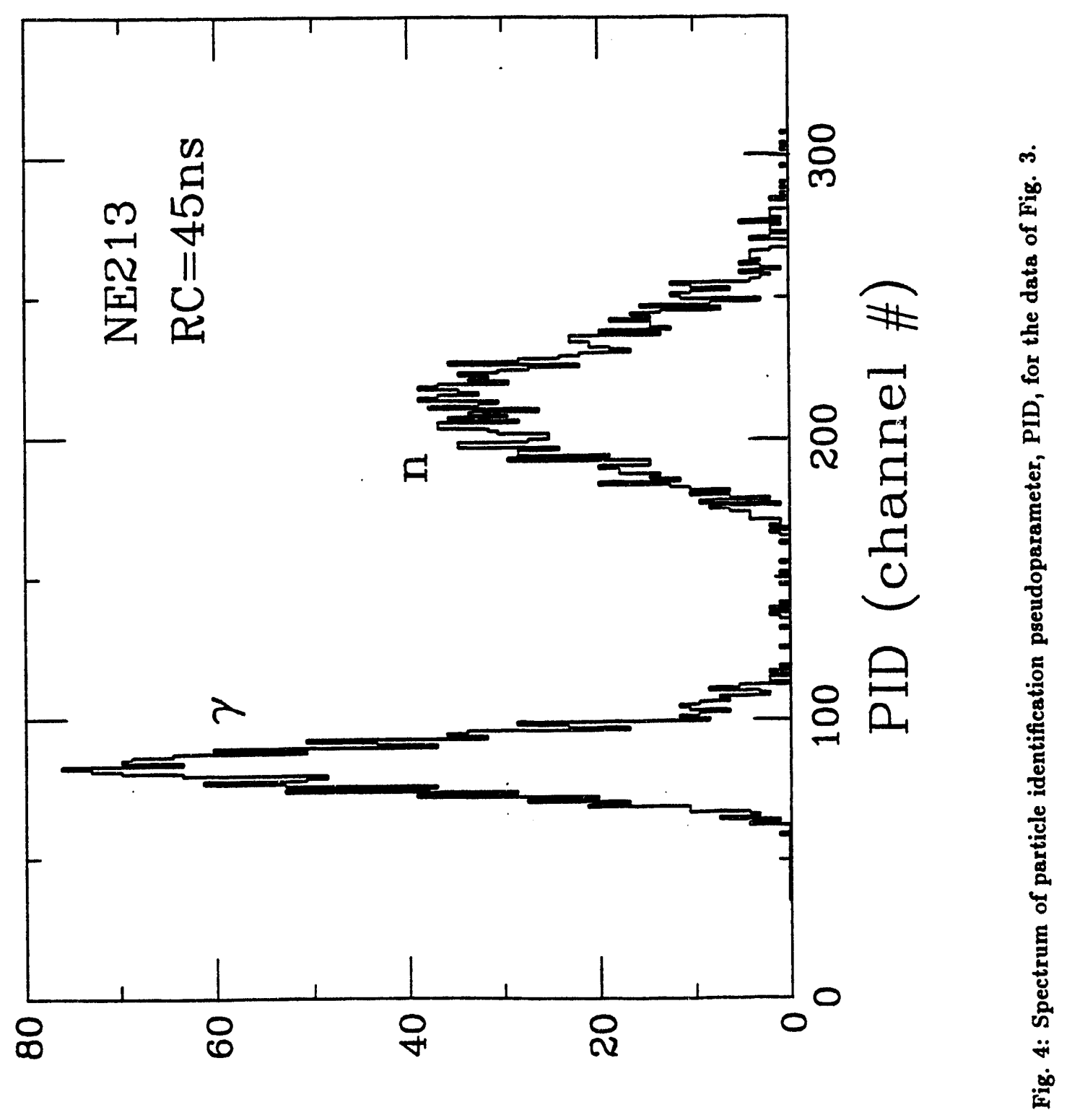

słunos 


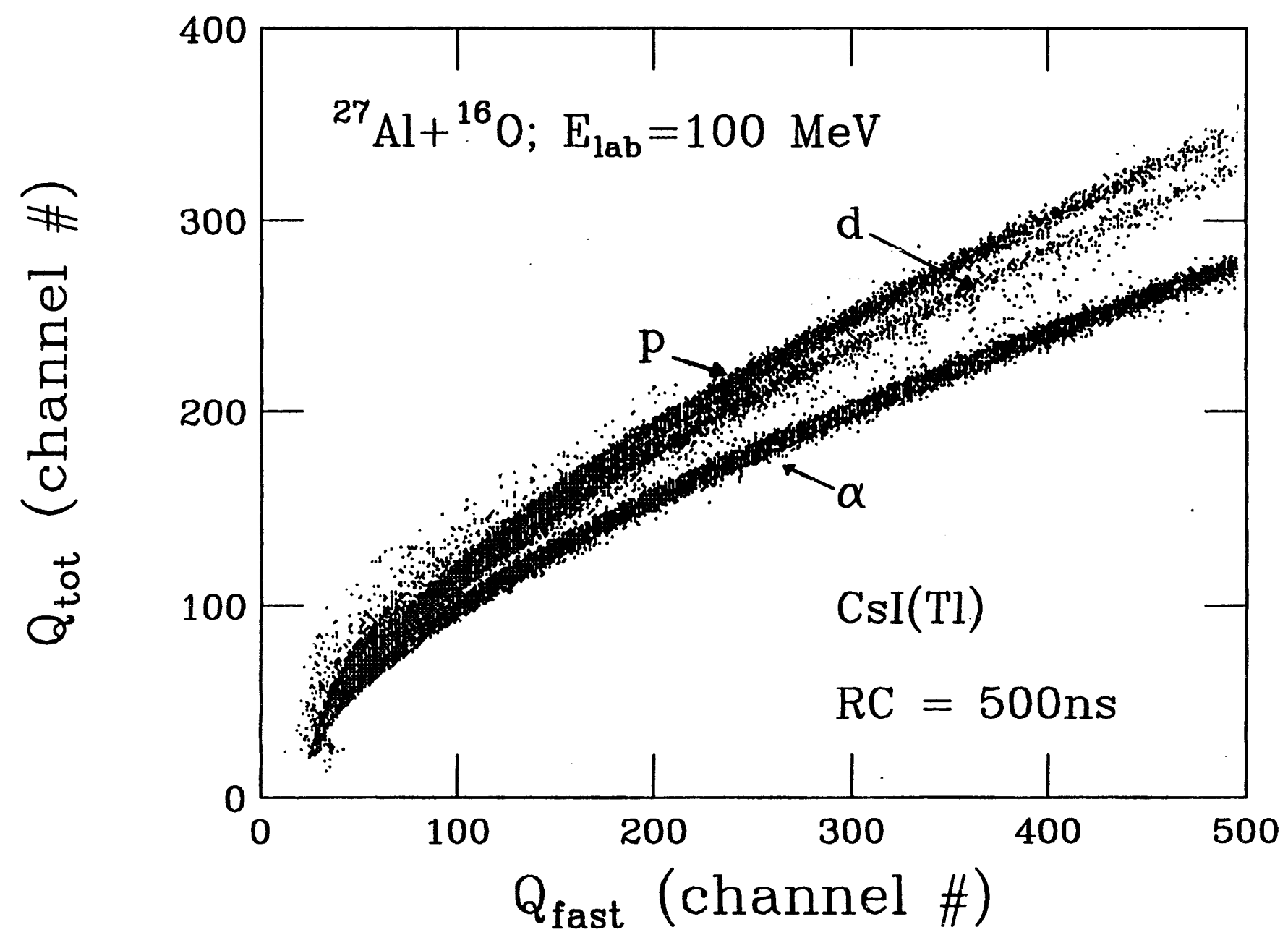

Fig. 5: Same as Fig. 3, except for a CsI(Tl) detector. 


\title{
A One-Transistor Preamplifier for PMT Anode Signals
}

\author{
J. Töke \\ Department of Chemistry and Nuclear Structure Research Laboratory, \\ University of Rochester, Rochester, New York 14627
}

\begin{abstract}
A simple one-transistor preamplifier stage has been designed for the anode pulses of a photomultiplier, to be operated in series with the voltage divider chain. The circuit, built into the PMT base and, hence, transparent to the experimenter, allows one to derive from the PMT base fast signals strong enough to be used without furher amplification for purposes of generating logical signals.
\end{abstract}


In many applications of the photomultiplier tubes, the signal derived from the anode is used for timing purposes only. When such a signal is sent over a cable to an associated electronic device, a $50 \Omega$ terminator resistor must be used at the input of the device in order to prevent reflections. As a result, the voltage signal at the input appears often too small to be used directly without an amplifier stage. For example, it has been found that the signals derived from the "standard" bases of the 14 PMT's of Rochester the neutron multiplicity meter (NMM) [1], must be amplified approximately by a factor of 40 in order to allow one to achieve a suitably low triggering threshold in conjunction with the available discriminators. One possible way of avoiding the necessity of using an (expensive) amplifier, is to employ, as close to the PMT base as possible, a fast, low-gain preamplifier. As the linearity of the resulting signal is not an issue in the timing applications, a simple one-transistor emitter follower stage close to the PMT base, or possibly built into the base, appears sufficient. In such a case the anode current pulse would generate in the large input impedance of the emitter follower a voltage signal (proportionally) larger than would be generated directly in the $50 \Omega$ terminator resistor at the input of the associated discriminator or other device. The amplitude of the output signal of the emitter follower, almost as large, can be driven into a $50 \Omega$ load without noticeable loss in amplitude. In an alternative picture, one might consider the transistor of the emitter follower as a device amplifying a part of the anode current of the PMT, with the amplified current sent to the $50 \Omega$ terminator resistor of the associated discriminator or other electronic device. This amplified current will then generate an amplified voltage signal at the input of the device, sufficient to trigger the device.

Fig. 1 shows a simple one-transistor implementation of the emitter-follower stage for driving the anode signals of a PMT. This implementation uses the PMT base current to power the emitter follower and, hence, does not require an additional lowvoltage power supply. As seen from Fig. 1, the fast pnp-type transistor T1 is biased using a voltage divider with an impedance of approximately $2 k \Omega$. Because of the much 
higher imput impedance of the transistor operated in this type of connection (load impedance times current gain $h_{f e}$ of the transistor T1), the input impedance of the preamplifier is in this case approximately $2 k \Omega$. This results in an output signal that is approximately 40 times larger than that generated directly in a $50 \Omega$ terminator. The resistors $\mathrm{R} 1$ and $\mathrm{R} 2$ in the bias chain of $\mathrm{T} 1$ are selected so as to obtain a quiescent base-collector voltage, i.e., the output saturation voltage, of approximately $16 \mathrm{~V}$. A $4.7 \mu \mathrm{F}$ electrolytic capacitor $\mathrm{C} 1$ between the transistor collector and the high voltage input serves as a virtual power supply for the preamplifier. The capacitor $\mathrm{C} 2$ provides for a $\mathrm{HV}$ decoupling and the resistor R4 prevents build-up of static charges at the output.

The bias divider resistors $\mathrm{R} 1$ and $\mathrm{R} 2$ can be calculated to achieve any desired voltage gain $G_{V}$ and saturation (collector) voltage $V_{\text {sat }}$, within allowed limits (see Eqs. 1-3). Assuming that the preamplifier of Fig. 1 is operated in series with a PMT voltage divider chain, at a chain current of $I_{\text {div }}$, and assuming, for sake of simplicity, that the current gain of the transistor $\mathrm{T} 1 h_{f e} \gg 1$ and that the connected load impedance is $R_{\text {load }}$, one can write

$$
\begin{gathered}
G_{V}=Z_{\text {input }} / R_{\text {load }}=\left(R 1^{-1}+R 2^{-1}+R 3^{-1}+h_{f e}^{-1} \frac{R_{\text {load }}+R 3}{R_{\text {load }} R 3}\right)^{-1} / R_{\text {load }} \\
V_{C}=V 1+R 2\left(\frac{V_{B}}{R_{B}}+\frac{I_{C}}{h_{f e}}\right) \\
I_{C}=I_{\text {div }}+\frac{V_{B}}{R 1},
\end{gathered}
$$

where $V_{B}=\left(I_{C} R 3+V_{E R}\right) / R 3$ and $V_{E B}$ is the emitter-base voltage, equal approximately $0.6 \mathrm{~V}$.

Equations 1-3 show that the limiting voltage gain $G_{V}$ that can be achieved in this scheme is equal to $h_{f e}$. These equations show also that it is possible to leave out the resistor $\mathrm{R} 1$ (i.e., $\mathrm{R} 1=\infty$ ) while changing $\mathrm{R} 2$ and $\mathrm{R} 3$ to define the Q-point of the transistor $\mathrm{T} 1$ and the voltage gain $G_{V}$ of the preamplifier within the allowed 
limits. The equations $1-3$ retain their validity, with $\mathrm{R} 1$ assumed infinitely large. In the scheme tested, it was found that without the resistor R1, an approximately $600 \mathrm{k} \Omega$ resistor $\mathrm{R} 2$ will ensure a collector-base voltage of $\mathrm{T} 1$ of $16 \mathrm{~V}$. This alternative circuit, without the resistor $\mathrm{R} 1$, uses fewer components, however, it has the disadvantage of being sensitive to the current gain $h_{f e}$ of the transistor T1.

Operation of the preamplifier stage in series with the PMT voltage divider chain alters the relative voltage of the PMT anode with respect to dynode voltages. Should this change (in the scheme of Fig. 1, by approximately 16V) be undesirable, it can be easily avoided by reducing appropriately the first resistor in the voltage divider chain.

Both types of preamplifier stages, with and without the resistor $\mathrm{R} 1$, have been built into the bases of the PMT's of the Rochester NMM [1] and have been successfully used directly with the associated discriminators. The voltage pulses generated by both types of preamplifiers on $50 \Omega$ terminator resistors had amplitudes up to $-16 \mathrm{~V}$ and rise times approximately $1 \mathrm{~ns}$.

This work was supported by the U.S. Department of Energy under grant number DE-FG02-88ER40414.

\section{Referenc :}

[1] S. P. Be: dwin, et al., in present Report 


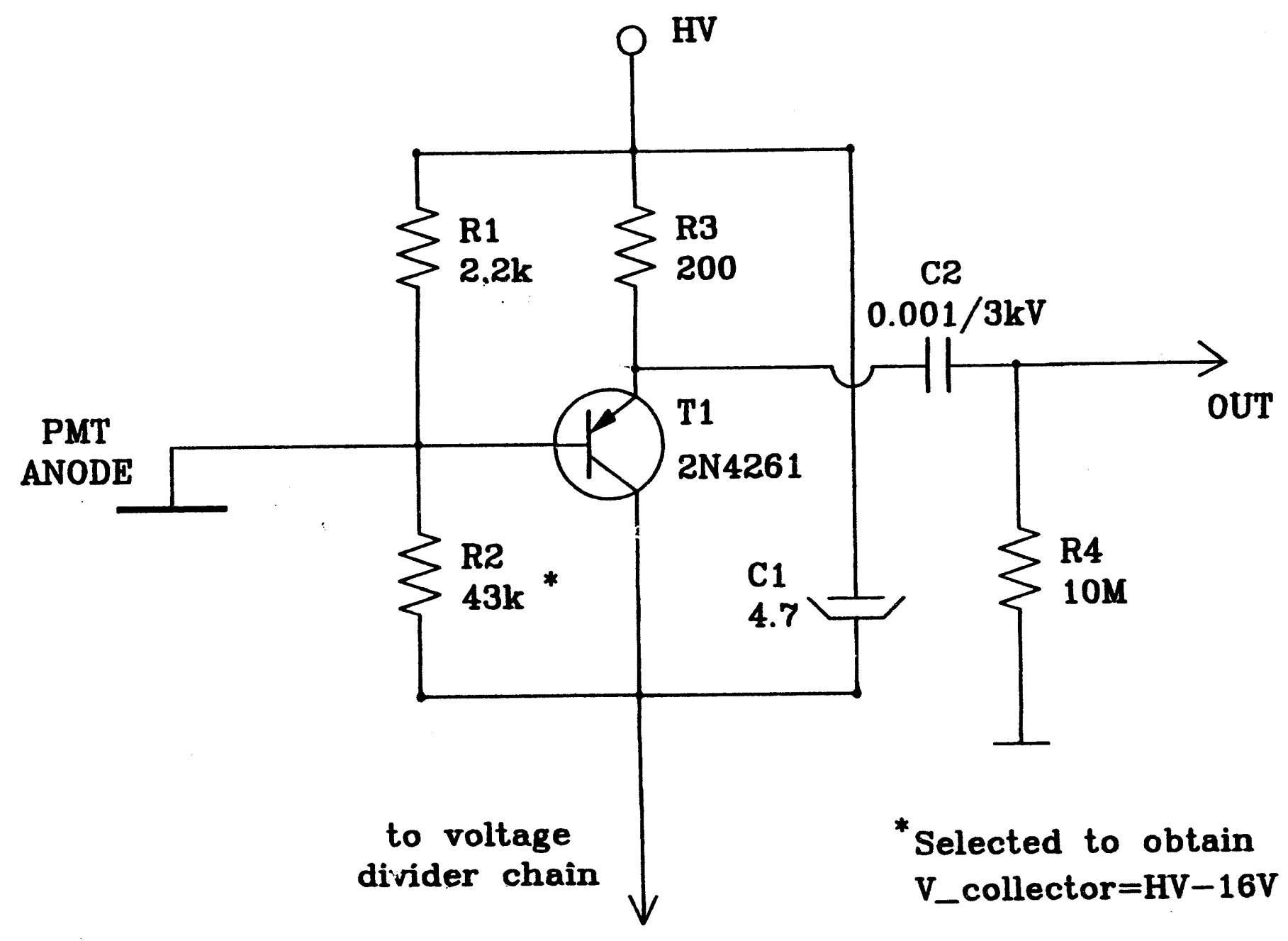

Fig. 1. Electronic circuit diagram of the fast one-transistor anode signal preamplifier. 


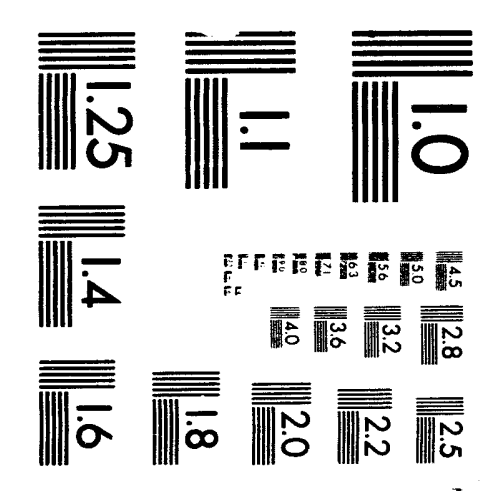



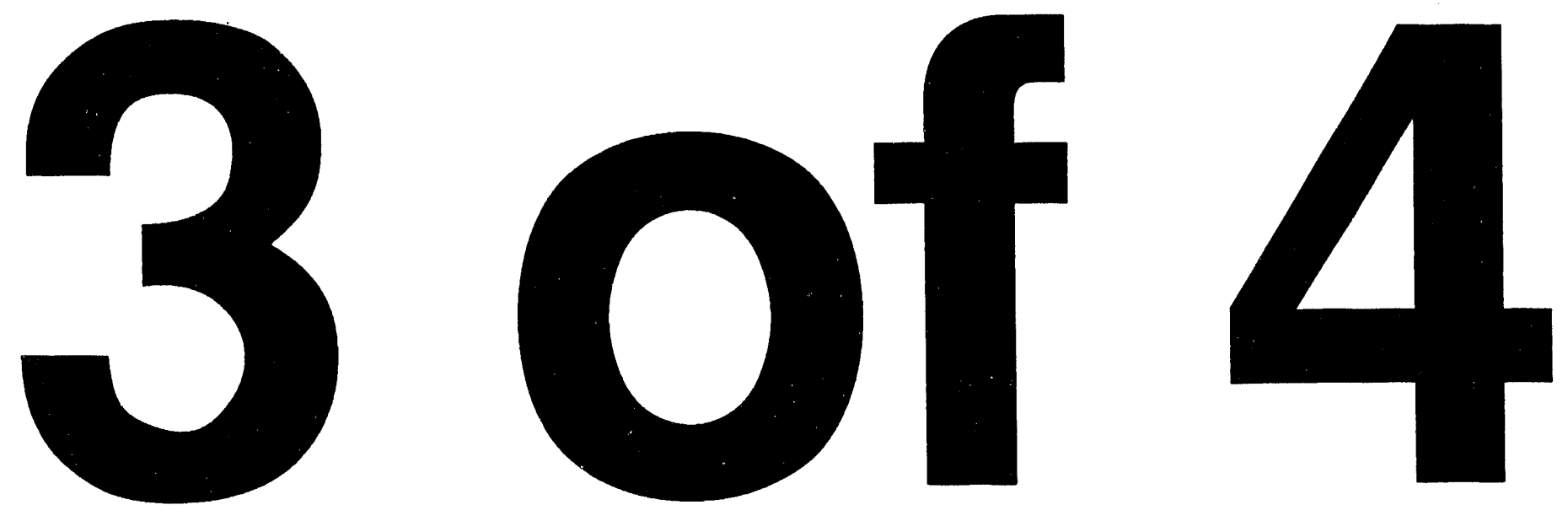


\title{
A Five-Channel Multistop TDC/Event Handler for the SuperBall Neutron Multiplicity Meter
}

\author{
J. Töke \\ Department of Chemistry and Nuclear Structure Research Laboratory, \\ University of Rochester, Rochester, New York 14627
}

\begin{abstract}
A CAMAC module has been designed and built to digitize information in the signal streams generated by the SuperBall neutron multiplicity meter (NMM) in response to reaction events. The module, implemented to a large extent in programmable array logic technology, is capable of recording event by event with $250 \mathrm{~ns}$ precision and with a $25 \mathrm{~ns}$ dead time, times of up to 512 signals for each of the five segments of the NMM separately, as well as for the NMM as a whole. Independently, the module counts multiplicities of signals arriving at each of the five inputs and multiplicities of logical OR signals for five selected combinations of inputs.
\end{abstract}

\section{INTRODUCTION}

Use of gadolinium-loaded scintillator neutron multiplicity meters (NMM) requires counting of signals within time intervals of the order of $100 \mu \mathrm{s}$, following the reaction or decay event of interest. Additionally, in many practical applications, it has been found desirable [1-3] to digitize and record the capture times of individual neutrons 
counted in each event. Such recording of neutron capture times allows one to optimize the counting interval after the experiment, in an off-line analysis, and allows one to monitor and correct for possible parasitic multiple triggering of the discriminators processing the NMM signals. Furthermore, capture time recording is especially usefull in experiments involving two or more "simple" NMM's [1] or one complex multisegment NMM's, such as SuperBall [4], where it allows one for a more precise rejection of cross-talk signals than it is possible using hardware solutions [5].

As a part of an effort to complete a compact and dedicated electronic instrumentation for the SuperBall neutron multiplicity meter under construction, a complex CAMAC module (5X512A) has been designed and built, combining multistop TDC's, scalers, and the controlling logic circuits to process signals from all five segments of the NMM. The 5X512A module is conceptually similar to the NMM event-handler modules of the 2 X64 series built in the past $[6,7]$ and used successfully in several experiments $[1,2,8]$. It achieves, however, somewhat better stability thanks to the use of Grey-coded internal representation of time, and achieves a significantly higher degree of compactness thanks to the use of a newer generation of more versatile FIFO memory chips and of more extensive than in earlier designs (2X64 series) use of programmable array logics (PAL) technology.

\section{PRINCIPLE OF OPERATION}

Principle of operation of the module can be understood from the block diagram shown in Fig. 1. The acquisition cycle is started by a (start) signal arriving at the LOGICS MASTER controller (in-house-programmed PALC22V10L by Cypress) through the START input of the module. In response to this start signal, the LOGICS MASTER performs the following tasks:

(i) starts the internal clock (implemented in a 74LS221 IC; tunable; set to $4 \mathrm{MHz}$ ),

(ii) enables the array of ten multiplicity COUNTERS (custom-programmed 
PALC22V10L) by removing the continuous RESET signal, (iii) enables the array of six FIFO memory IC's (CY7C421 by Cypress) by removing the continuous RES (reset) signal, (iv) disables LOGIC MASTER's start input to prevent retriggering, (v) sends a "busy" signal to the front-panel visual LED indicator $B$, (vii) sets a "module busy" signal at the front-panel DT (dead time) output.

The clock signal is applied to the inputs of the binary TIMER COUNTER and of the GREY-CODER (both custom-programmed PALC22V10L), the two IC's programmed to generate a 9-bit Grey-coded timer data. The 9-bit output of the GREYCODER is sent to the "CLOCK-WRITE" bus of the FIFO's for storage in the respective FIFO's upon arrival of the SI (shift in) signals.

The signals applied to the (five) STOP inputs are applied to the "STOP"- INPUT BUS of the multiplicity COUNTER array. Five of these counters are programmed to respond to a signal at one particular STOP input, four counters respond to binary logical OR's of signals at adjacent STOP inputs, while one counter responds to a signal at any input (logical OR of all inputs). The multiplicity COUNTERS count (in binary representation) recognized signals received through the input bus. An overflow protection programmed into these counters prevent them from cycling through zero upon reaching 511. Additionally, in response to a valid input signal, each multiplicity counter generates an output signal that is applied to the SI (shift in) input of the associated FIFO (the four binary logical "OR" multiplicity counters have no associated FIFO's). These latter signals cause respective FIFO's to store in their consequtive memory locations (up to 512) the Grey-coded 9-bit representation of the time in the cycle, present on the "CLOCK-WRITE" input bus of the FIFO array. The acquisition cycle continues until the binary TIMER COUNTER reaches a preset value determined by the set of 8 switches (TIMER PRESET), sensed by the TIMER COUNTER programmed IC. Upon reaching the preset value, the TIMER 
COUNTER issues an EOA (end of acquisition) signal for the LOGICS MASTER. In response to this EOA signal, the LOGICS MASTER performs the following tasks: (vii) disables inputs of the multiplicity counters and, hence, disables also the FIFO's by preventing them from receiving further "shift-in" (SI) pulses, (viii) sends a "done" signal to the front-panel INT OUT output (six different formats of this signal are available, selected by a set of three jumpers JMP - see Section III), (ix) generates a LAM signal to be sent to the CAMAC bus, if the LAM is enabled.

Upon the termination of the acquisition cycle, the module stores in its five FIFO's Grey-coded binary representations of times of arrival of consequtive signals at the associated STOP inputs (neutron capture times, in the particular application to an NMM) and stores in its ten multiplicity COUNTERS numbers of stop signals (neutron multiplicities) received at the associated STOP inputs.

Subsequent readout of the COUNTERS and FIFO's is achieved through decoding of CAMAC N F(0) or NF(2) functions using the custom-programmed CAMAC DECODER (PALC22V10L) and subaddress demultiplexer (ADDRESS DEMUX) (GAL16V8A by Lattice). The Grey-coded contents of the FIFO memory locations are converted "on the fly" back to the regular binary representation by the customprogrammed GREY-DECODER (PALC22V10L), and then placed, through a buffer, on the CAMAC "READ" bus.

Upon completion of the readout, the module is cleared by applying CAMAC $\mathrm{N}$ F(9) function or a Clear command. The module can be also cleared by an external "fast clear" signal applied to the front-panel CLEAR input any time after the module has been started. Whether received through the CAMAC crate or through the frontpanel CLEAR input, the "clear" signal causes the LOGICS MASTER to perform the following tasks:

(x) set the reset signals on the RST inputs of the TIMER COUNTER and GREYCODER, 
(xi) set the reset signals on the control buses of the FIFO and multiplicity counter arrays,

(xii) remove the "disable" signal from the 2-bit control bus of the multiplicity counters (the counters remain, however, disabled due to the presence of the continuous "reset" signal),

(xiii) remove the "busy" signal from the DT output, (xiv) remove the "busy" signal from the LED indicator, (xv) clear the LAM register.

Upon processing a "clear" signal the module is ready to accept a next event.

\section{CAMAC COMMANDS AND FUNCTIONS}

CAMAC commands are decoded using custom-programmed CAMAC DECODER (PALC22V10) and ADDRESS DEMUX (GAL16V8 by Lattice).

\section{A. CAMAC commands}

Command Response of the module

I

- Inhibit; the Start input is disabled and a "busy" signal (NIM) is generated and sent to the front-panel Lemo jack DT.

C - Clear; all internal registers are cleared. The LOGICS MASTER is reset and the module is ready to accept the next event.

- Initialize; The module is cleared (see Clear command "C") and LAM is disabled 


\section{B. CAMAC functions}

N F(0) $A(k)$ or $N F(2) A(k)$, where $k=0$ - 15 (see table below) - read internal registers of the module:

k register to be read

0,2,4,6,8 - FIFO's associated with inputs $1-5$, respectively.

10 - FIFO associated with a logical OR of signals at all five inputs

$1,3,5,7,9$ - multiplicity counters associated with inputs $1-5$, respectively.

11 - multiplicity counter associated with a logical OR of signals at all five inputs.

12 - 15 - multiplicity counters associated with binary logical OR's of signals at inputs 1 and 2,2 and 3,3 and 4, and 4 and 5, respectively.

N F(10) $A\left({ }^{*}\right)$ - Clear LAM; requires S2.

N F(26) $A\left(^{*}\right)$ - LAM enable; requires $S 2$.

N F(24) $A\left({ }^{*}\right)$ - LAM disable; requires S2.

N F(9) $A\left({ }^{*}\right) \quad$ - Clear module; requires $S 2$.

In response to every valid command or function, module generates $\mathrm{X}$ and $\mathrm{Q}$ signals and sends these to the respective CAMAC control lines.

\section{SPECIAL FEATURES}

\section{A. Diagnostics Mode}

The frequency of the internal CLOCK can be adjusted (within $\pm 10 \%$ ) using the CLOCK trimpot. To allow one monitoring of the clock frequency at the front-panel DT output, the module is set to a diagnostics mode by applying a continuous signal (NIM) to the CLEAR input. Upon removal of this signal, the module returns automatically to the standard mode. 


\section{B. Interrupt-Out Signal}

The module signals end of an acquisition cycle by an output signal at the INT OUT front-panel connector. This signal can be then used, for example, to control start of the readout cycle. Six formats of this signal are possible, selectable through setting of three jumpers at MODE SEL (two active positions of the header labeled J1 on the module printed circuit board) and at the INT OUT output (labeled J2 on the printed circuit board).

Two first positions (leftmost) at J1 serve as storage "bins" for the unused jumpers. Jumper at the third position of $\mathrm{J} 1$ allows one to select between pulsed (250 ns) (jumper in) and continuous-level (jumper out) output signal. Jumper at the fourth (rightmost) position of $\mathrm{J} 1$ allows one to select between the straight, i.e., positive for TTL and negative for NIM, (jumper out) and inverted (jumper in) output signal. Jumper J2 allows one to select between TTL-compatible (jumper in upper position) and NIM-compatible (lower position) signal.

\section{Length of Counting Gate}

The acquisition time for events (counting gate) can be preset with 8-bit precision using the "keyboard" type octal DIP switch TIMER PRESET. Switch "open"(up) sets the respective bit to 1 , while switch closed zeroes the bit. For example, all switches up represent binary 511 , i.e., the counting gate of approximately $128 \mu \mathrm{s}$.

\section{SPECIFICATIONS}

Start - common input, NIM, $50 \Omega \mathrm{hm}$ impedance; min. $25 \mathrm{~ns}$.

Stop - five inputs, NIM, $50 \Omega \mathrm{hm}$ impedance; $\min 25 \mathrm{~ns}$; up to 512 signals digitized per event per input.

Fast Clear - common input, NIM, $50 \Omega \mathrm{hm}$ impedance; min $30 \mathrm{~ns}$. Continuous 
Clear causes module to switch to diagnostics mode.

DT - output, NIM; in normal mode, signal is present at this output whenever the module is inhibited from accepting next event. In diagnostic mode (see Section 4, and Fast Clear above), internal clock signal is output.

Int Out - output, signals end of acquisition NIM or TTL, straight or inverted, continuous level or $250 \mathrm{~ns}$ pulse; jumper selected (see Section IV).

Conversion - adjustable (see Section IV), set to $250 \mathrm{~ns} / \mathrm{channel}$.

Time offset - one channel, i.e., zero time appears in channel 1.

Gate length - switch-selected with 8-bit precision, up to 511 clock cycles (see Section IV).

Dead time $-<30$ ns, i.e., two stop pulses apart at least by 30 ns can be resolved.

Data - for each of the five channels, the multiplicity and up to 512 times are recorded with 9-bit precision for the subsequent readout under the CAMAC protocol. Additionally, times and multiplicity are recorded for a logical OR signal for all inputs, and further, multiplicities are recorded for five different logical binary OR's of signals at inputs 1 and 2,2 and 3,3 and 4, and 4 and 5, respectively.

Red LED - valid CAMAC command received (pulsed) or module is receiving CAMAC inhibit (continuous).

Green LED - Start signal received (pulsed) or module not cleared (continuous). Packaging - in conformance with CAMAC standard, single-width module. Power $\quad-\quad+6 \mathrm{~V}$ at $800 \mathrm{~mA} ;-6 \mathrm{~V}$ at $150 \mathrm{~mA}$.

This work was supported by the U.S. Department of Energy under grant number DE-FG02-88ER40414. 


\section{References}

[1] D. Pade, et al.,Phys. Rev. C43, 1288 (1991).

[2] J. L. Wile, et al., Phys Rev. C47, 2135 (1993).

[3] R. Schmidt, et al.,

[4] X. Zhao, et al., this Progress Report.

[5] M. Morjean et al., Nucl. Phys. A524, 179 (1991).

[6] J. Tõke, USDOE Progress Report, COO-3496-82, 279 (1987).

[7] J. Tõke and S. A. Baldwin, USDOE Progress Report, DOE/ER/40414-4, 218 (1989).

[8] B. Lott, et al., Phys. Rev. Lett. 68, 3141 (1992). 


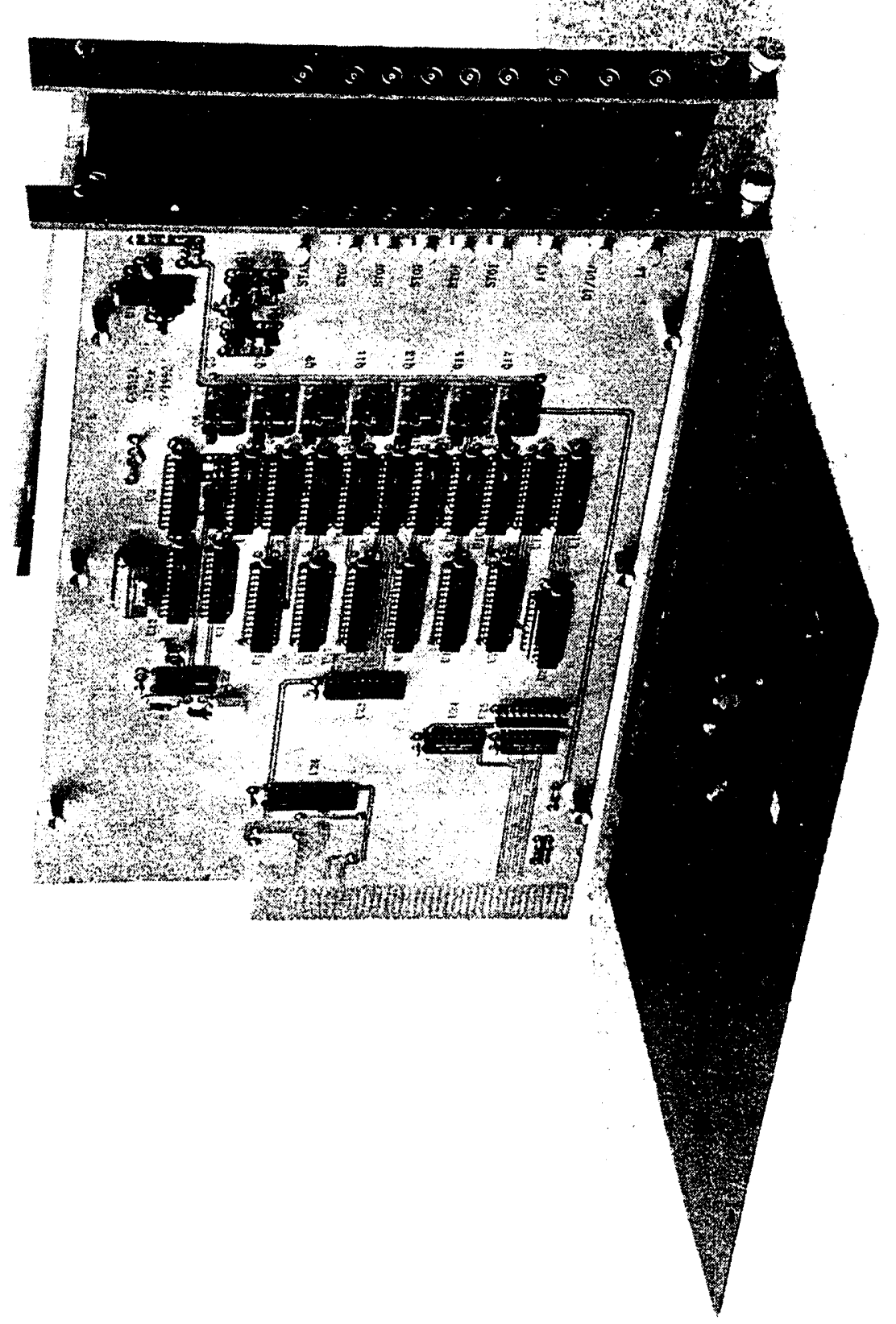




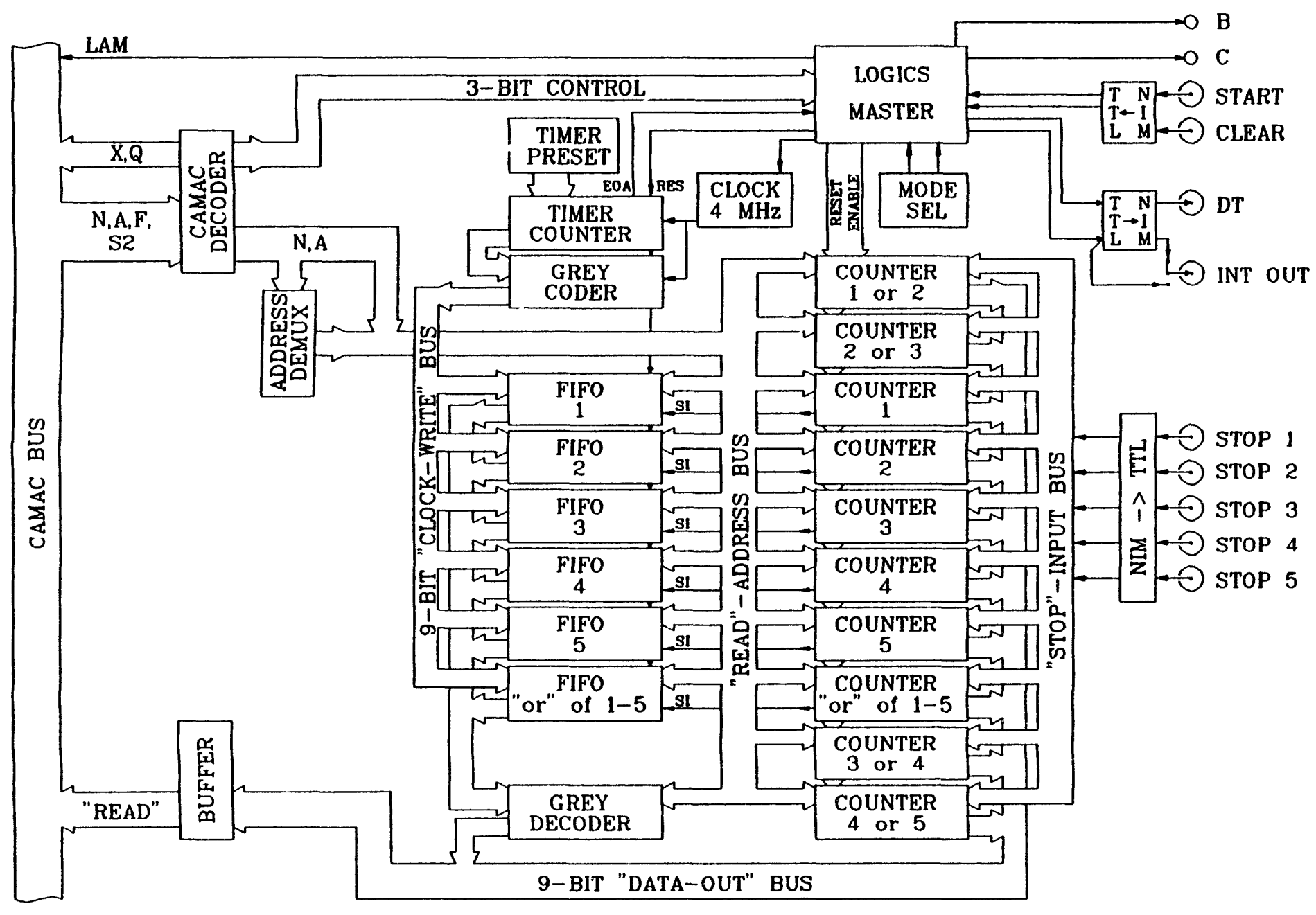

FIG. 1. Block diagram of the 5X512A multistop TDC/event handler. 
Construction of the SuperBall - a 16,000-liter

Neutron Detector for Calorimetric Studies of Intermediate-Energy Heavy-Ion Reactions

\author{
X. Zhao, S. P. Baldwin, W. U. Schröder, and J. Töke, \\ Department of Chemistry and Nuclear Structure Research Laboratory \\ University of Rochester, Rochester, NY 14627, USA
}

\begin{abstract}
A large-volume, multi-segment $4 \pi$ Neutron Calorimeter (SuperBall) has been designed at the University of Rochester for studies of various aspects of nuclear collisions at intermediate bombarding energies. The design of the SuperBall allows it to accommodate complex setups of the charged-particle detectors, such as Dwarf or MiniBall $4 \pi$ arrays, and hence, allows one to carry out experiments in which both neutrons and charged products are measured with a $4 \pi$ angular coverage, as well as with multi-hit capability. Status of the SuperBall, project, is discussed.
\end{abstract}




\section{INTRODUCTION}

Studies of heavy-ion reactions at bombarding energies of a few $\mathrm{MeV} /$ nucleon above the interaction barrier have benefited greatly from measurements of neutron multiplicities [1]. In particular, for damped reactions, the multiplicities of emitted neutrons were found to be strongly correlated with total kinetic energy loss $\mathrm{E}_{\text {lose }}[2,3]$, and hence to provide a rather direct measure of the total excitation energy of the system. For fusion reactions, on the other hand, where the excitation energy is fixed, neutron multiplicities were found useful in understanding thermal properties of composite systems [4].

Correlations between the neutron multiplicity and the degree of energy dissipation clearly persist also at intermediate bombarding energies, i.e., near the Fermi energy regime, which makes measurement of the neutron multiplicities a much desired complement to charged particle measurements. A realistic evaluation of the energy dissipated in an intermediateenergy heavy-ion collision is difficult to achieve based solely on measurements of the charged particles. On the other hand, even a moderately accurate knowledge of the multiplicity and average kinetic energy of the emitted neutrons allows one to reconstruct the excitation energy of an event with only a small correction for charged-particle emission [5-7]. This fact makes neutron calorimetry a highly efficient, versatile, simple, and elegant tool for the studies of dynamics of intermediate-energy heavy-ion reactions and properties of the transiently formed nuclear systems characterized by unusually high temperatures, spins, and perhaps densities.

With these benefits from the measurement of neutron multiplicities in mind, a program was started at the University of Rochester to design, construct, and put into operation at the National Cyclotron Laboratory of the Michigan State University a large-volume, segmented neutron calorimeter, termed SuperBall, that can be operated in conjunction with large arrays of charged particle detectors in explorations of nuclear phenomena occurring during intermediate-energy heavy-ion collision. Its elegant principle of operation is based on the method $[8,9]$ of statistically spreading in time response of the detector to neutrons, 
through processes of the thermalization and subsequent capture by Gd nuclei present in the active volume of the detector. Through this spread in time, the detector gains the multi-hit capability, allowing one to count one-by-one, at different times, many neutrons that have simultaneously entered the active volume of the detector. In the following sections, status of the SuperBall project, nearing the completion, is reviewed.

\section{MECHANICAL DESIGN}

General design and performance considerations of the SuperBall neutron detector are discussed in detail in [10], where they are supported by the results of series of Monte Carlo simulations. Based on these considerations, as well as on considerations of cost effectiveness and engineering feasibility, a final mechanical design has been chosen that in essence coincides with the one considered in [10]. The array of five constituent tanks of the SuperBall has been built according to this final design at the FAB-ALLOY Company in Jackson, Michigan.

Mechanical design of the SuperBall is shown in Figs. 1- 3. As seen from these figures, the SuperBall consists of a mutually "docking" array of five axially-symmetrical tanks of toroidal topology mounted on mobile undercarriages. Geometrical parameters of the tanks are shown in Table I. Tanks are welded from sections of stainless steel pipes and sheets, 1/4" thick, formed to cylindrical, conical, or spherical shapes. To the outer shells of the tanks are welded photomultiplier ports designed to accept sealed glass windows separating the active scintillator fluid from the photomultipliers. The two "end" tanks have eight ports each, while the three inner ones have twelve ports each. The cylindrical inner shell of the tanks allows for the passage of the beam pipe and accommodation of a 35"-diameter 45"-long scattering chamber. Additionally, the "interface" walls between the tanks \#2 and \#3 are formed so as to to accommodate two cable ducts, TV Camera target viewing port and the scattering chamber support structure (see Fig. 5). Part of the support structure serves also as a scattering chamber pumping duct.

Inner walls of the five SuperBall tanks are lined with $1 / 32$ "-thick teflon sheets to enhance 
the light collection efficiency. To this end, overlapping sections of teflon sheets of proper shapes and sizes are attached to the stainless steel shell using 1/8"-diameter stainless steel studs spot-welded to the shell.

The tanks are welded to adjustable support structures mounted on movable undercarriages. In order to be able to make the undercarriages sufficiently long to guarantee static stability of the tank-undercarriage assemblies, two different widths of undercarriages are used, which allow for their interlacing in the "operating" configuration of the SuperBall, i.e., when the tanks are moved together.

\section{DESIGN OF THE SCATTERING CHAMBER}

The design of the scattering chamber is shown in Figs. 3-5. The chamber is made of stainless steel and consists of a stationary ring and two removable end caps. The stationary ring is mounted on a support structure attached solidly to the floor. The upper part of the support structure is an 8 "-diameter nickel-plated steel pipe serving also as a pumping duct for a cryopump. The stationary ring provides mechanical support for major components of the experimental setup housed by the scattering chamber. In Figs. 4 and 5 are shown cross sections of the scattering chamber housing the MSU MiniBall assembly. From the side wall of the ring extrude two pipes serving as cable ducts. Additionally, to the side wall of the ring are attached a target mechanism and a target viewing window.

The two end caps of the scattering chamber can be dismounted from the stationary ring, allowing one to gain access to the chamber. Each of these end caps consists of a section of a cylinder and a spherical end piece connecting through a flange to the beam pipe. Provisions are made for measuring vacuum in the chamber.

\section{ELECTRONIC SIGNAL PROCESSING}

The response of the SuperBall to neutrons, $\gamma$-rays and other types of nuclear radiation capable of penetrating the active volume of the SuperBall (e.g. cosmic rays) consists of 
electrical signals picked from the anodes and last dynodes of the photomultiplier tubes viewing the active volume. These "raw" signals are processed to derive two types of "refined" detector signals - analog signals representing the magnitude of the light output and logical signals representing acts of detection. Subsequently, streams of these refined signals are digitized and read into the data acquisition system.

The SuperBall uses a total of 52 PMT's distributed 8-12-12-12-8 over its five constituent tanks. Large number of PMT's makes it highly desirable both from the price and setup time considerations to develop a dedicated integrated electronics modules for processing of the PMT signals. Such modules have been designed and are described in more detail below.

\section{A. Integrated PMT Anode Signal Processing}

Logical signals of the SuperBall are derived from the photomultiplier anode signals and processed using custom designed integrated electronics modules. A Five-Channel Multistop TDC/Event Handler for the SuperBall detector, described in [11] has been designed, built and successfully used in experiments with the Rochester Medium Volume Neutron Multiplicity Meter RedBall [12] As described in Ref. [11], this CAMAC module is capable of counting multiplicities and of digitizing arrival times of up to 512 logical signals (neutron capture times) per event and per channel. The suitable logical signals of the devices such as the SuperBall are usually derived from the anode signals of the PMT's using standard electronics "building blocks" such as amplifiers, discriminators and logical AND and OR gates. Here a design of an integrated electronics module is described capable of performing all essential functions of the electronics setups consisting of such standard building blocks at a fraction of cost. Furthermore, the designed Integrated Logic Module requires very little setup time and is characterized by high flexibility in the selection of PMT "firing" patterns to be accepted for acquisition.

The block diagram of the Integrated Logic Module is shown in Fig 6. Signals from the active PMT bases [13] are applied through the gain-matching attenuators to the inputs of fast 
comparators (LeCroy MVL407). Output signals from these comparators, produced whenever the input signal exceeds the adjustable threshold value, are stretched by monostable multivibrators and applied to the address input pins of a fast (20 ns) EPROM (Cypress CY7C263 - 8192x8 bit). The EPROM is programmed so that valid PMT firing patterns (input addresses of the EPROM) are signaled by the presence of the respective bit in the EPROM output lines. Since the EPROM output consists of 8 bits, 8 different modes corresponding to different sets of valid input patterns can be programmed into the EPROM. Furthermore, the 13-th address bit, unused for the comparator signals (SuperBall has at most 12 PMT's per segment) can be used to extend the number of preprogrammed modes of operation to 16. Subsequently, a particular operation mode is selected by four switches addressing a 8-to-1 multiplexer (selection of an EPROM output bit) and the 13-th EPROM address bit (selection of the memory half). The signal from the multiplexer is used to generate a standard-length TTL signal, which is then level-converted to a NIM signal and applied to the output jack of the module.

Use of the EPROM in selection of valid PMT firing patterns offers a flexibility virtually unattainable in setups using commercial logic AND and OR gates. Not only with a flip of a few switches can the mode of operation be dramatically altered to any of the preprogrammed options, but with a reprogramming of the EPROM any conceivable mode can be achieved fast without changes in the hardware setup.

\section{ANALOG DYNOD SIGNAL PROCESSING}

Dynode signals are used to measure light output produced in any single act of detection and were shown to be a valuable tcol in measuring the total kinetic energy deposited in the scintillator fluid by neutrons emitted in a single reaction event. A procedure, earlier developed for the Rochester RedBall detector has been tentatively adopted for the SuperBall. This procedure consists in summing the gain-matched dynode signals of all PMT's in one constituent segment of the SuperBall and current-integrating the summed signal using a 
current-integrating ADC.

\section{SUMMARY}

Construction of the SuperBall basic mechanical structure nears completion. Work is being currently concentrated on other mechanical elements supplementing this basic structure such as the scattering chamber, scintillator fluid handling system, and a cryopump system.

Also, work is being conducted in parallel on completing the elements of the electronics setup to handle the SuperBall signals, and in particular on completing the Integrated Logic Modules described in Section IV.A.

The SuperBall is expected to be operational by December 1993.

This work was supported by the U.S. Department of Energy Grant No. DE-FG0288ER40414 and by the U.S. Department of Energy University Research Instrumentation Grant No. DE-FG05-91ER79048. 


\section{REFERENCES}

[1] W. U. Schröder and J. R. Huizenga, Treatise on Heavy-Ion Science, (D. A. Bromley, Editor), Plenum Press (New York and London, 1984), Vol.2,pp113-726; and references therein.

[2] D. Hilscher et al., Phys. Rev. C20, 575 (1979).

[3] D. Pade et al., Phys. Rev. C43, 1288 (1991).

[4] J. L. Wile et al., Phys Rev. C47, 2135 (1993).

[5] D. Guerreau, in Nuclear Matter and Heavy-Ion Collisions, M. Soyer, H. Flocard, B. Tamain, and M. Porneuf, editors, (Plenum Publ. Corp., 1989),p.187.

[6] B. Lott et al., Phys. Rev. Lett. 68, 3141 (1992).

[7] U. Jahnke et al., Proc. XX International Summer School Nucl. Phys., Mikolajki, 1988, Report HMI-B 463, 1988.

[8] B. C. Diven et al., Phys. Rev. 101, 1012 (1956).

[9] U. Jahnke et al., Lect Notes in Physics 178, 179 (1983).

[10] X. Zhao et al., Progress Report DOE/ER/40414-5, 190 (1992).

[11] J. Tõke, A Five-Channel Multistop TDC/Event Handler for the SuperBall Neutron Multiplicity Meter, this report.

[12] S. P. Baldwin et al., The Rochester 960-liter Neutron Multiplicity Meter, this report.

[13] J. Tõke, A One-Transistor Preamplifier for PMT Anode Signals, this report. 


\section{FIGURES}

FIG. 1. Perspective view of the SuperBall assembly in "operating" configuration.

FIG. 2. Computer rendering of the perspective view of the SuperBall in "servicing" configuration with the scattering chamber exposed.

FIG. 3. Vertical cross-section of the scattering chamber and of the supporting structure - side view.

FIG. 4. Vertical cross-section of the SuperBall assembly and scattering chamber with the MSU MiniBall installed - side view.

FIG. 5. vertical cross-section of the SuperBall assembly and scattering chamber with the MSU MiniBall installed - front view.

FIG. 6. Block diagram of the Integrated Logic Module handling the PMT anode signal streams from one segment of the SuperBall. 
Table 1. Physical parameters of the SuperBall

\begin{tabular}{||c|c|c|c|c|c||}
\hline \hline & $\begin{array}{c}\text { Angular } \\
\text { Coverage } \\
\text { (degree) }\end{array}$ & $\begin{array}{c}\text { Solid Angle } \\
\text { Coverage } \\
(4 \pi \text { sr })\end{array}$ & $\begin{array}{c}\text { Effective } \\
\text { Thickness } \\
(\mathrm{cm})\end{array}$ & $\begin{array}{c}\text { Volume } \\
\left(\mathrm{m}^{3}\right)\end{array}$ & $\begin{array}{c}\text { Empty } \\
\text { Weight } \\
(\mathrm{kg})\end{array}$ \\
\hline Segment 1 & $137-180$ & 0.14 & 102 & 1.91 & $4.8 \times 10^{2}$ \\
Segment 2 & $90-137$ & 0.34 & 102 & 4.25 & $1.11 \times 10^{3}$ \\
Segment 3 & $60-90$ & 0.25 & 102 & 3.49 & $1.10 \times 10^{3}$ \\
Segment 4 & $32-60$ & 0.17 & 102 & 4.21 & $1.09 \times 10^{3}$ \\
Segment 5 & $0-32$ & 0.08 & 127 & 2.46 & $5.3 \times 10^{2}$ \\
\hline Whole Detector & & 0.98 & & 16.31 & $4.30 \times 10^{3}$ \\
\hline
\end{tabular}




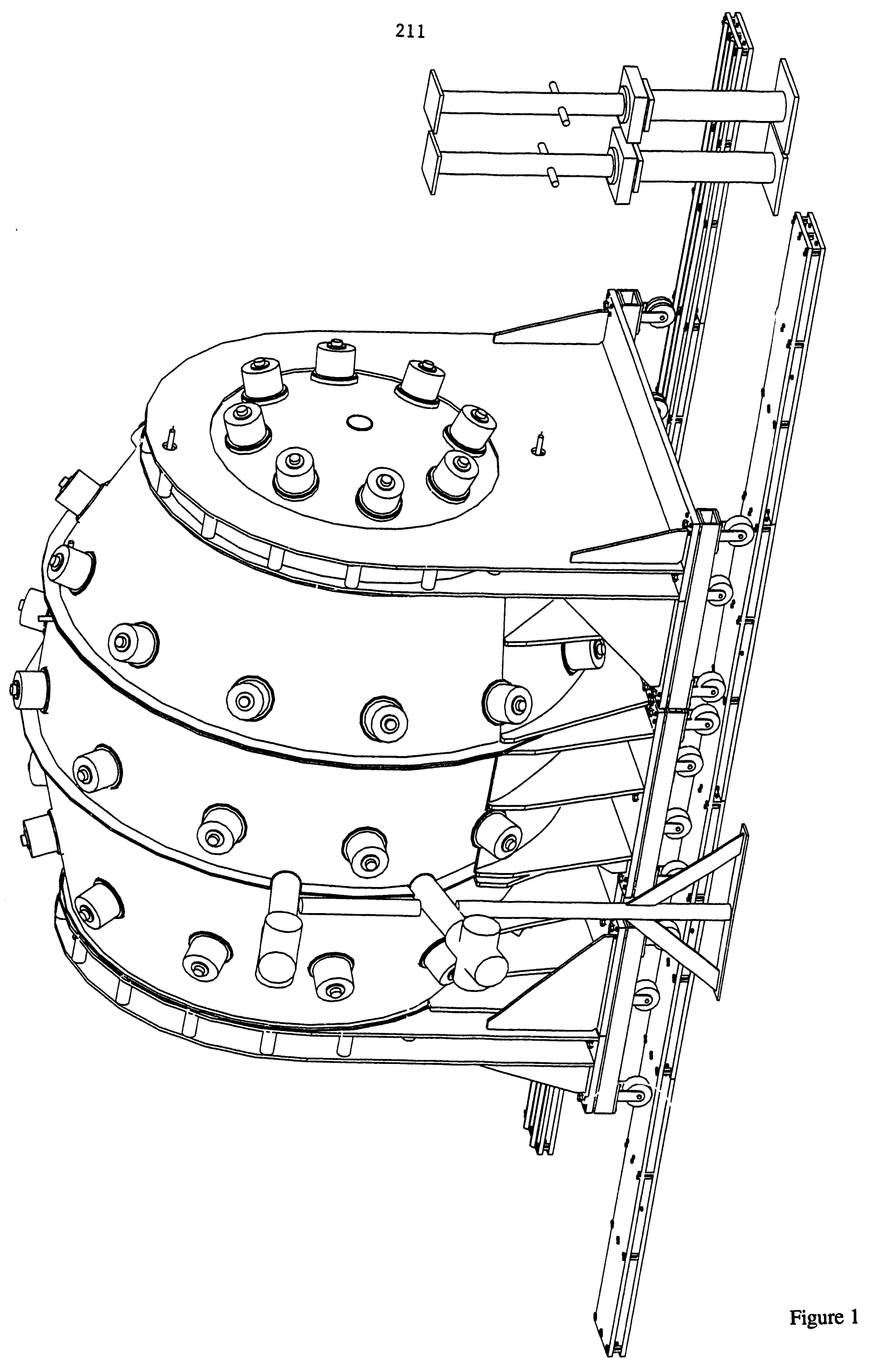




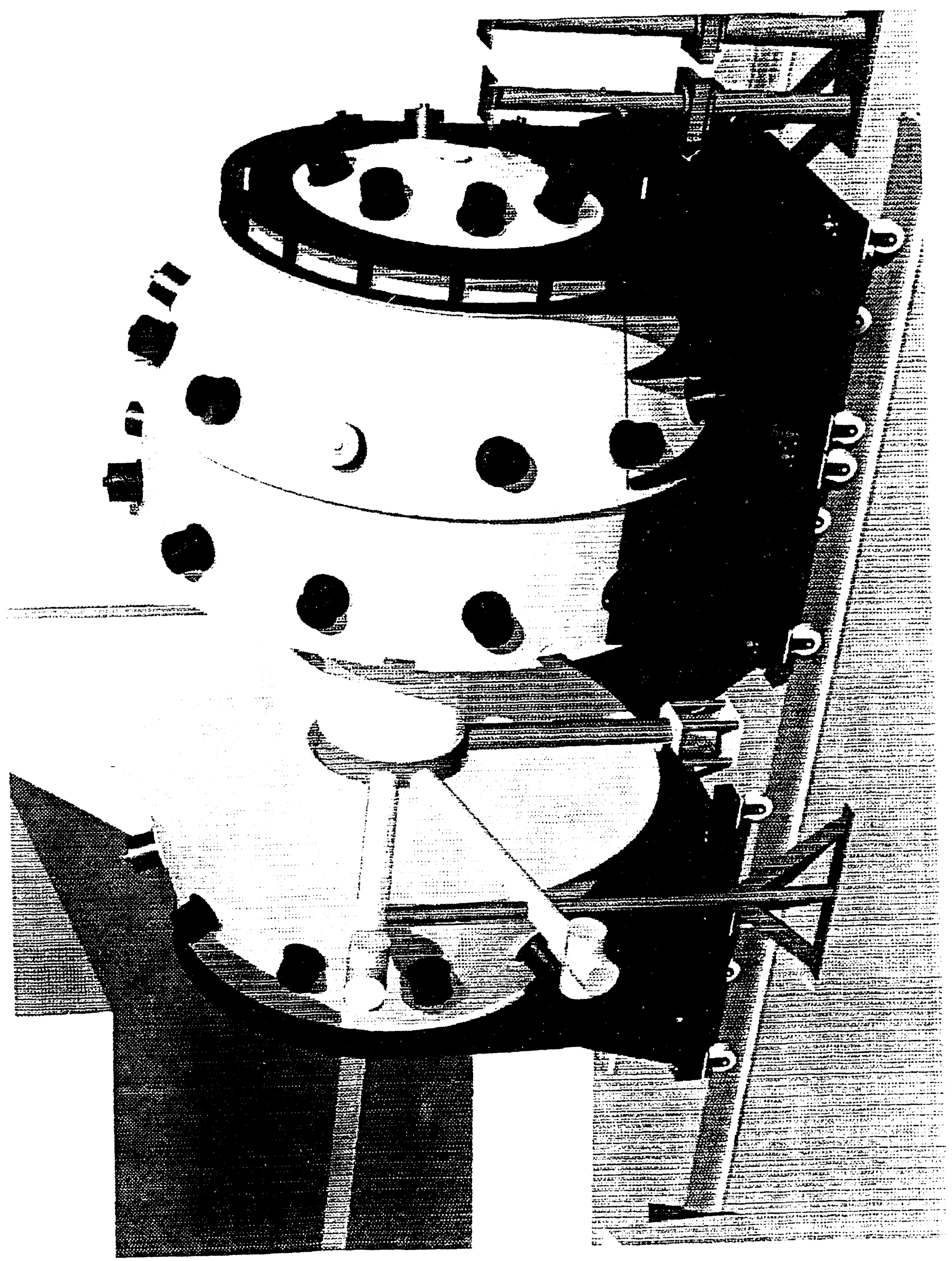

Figure 2 


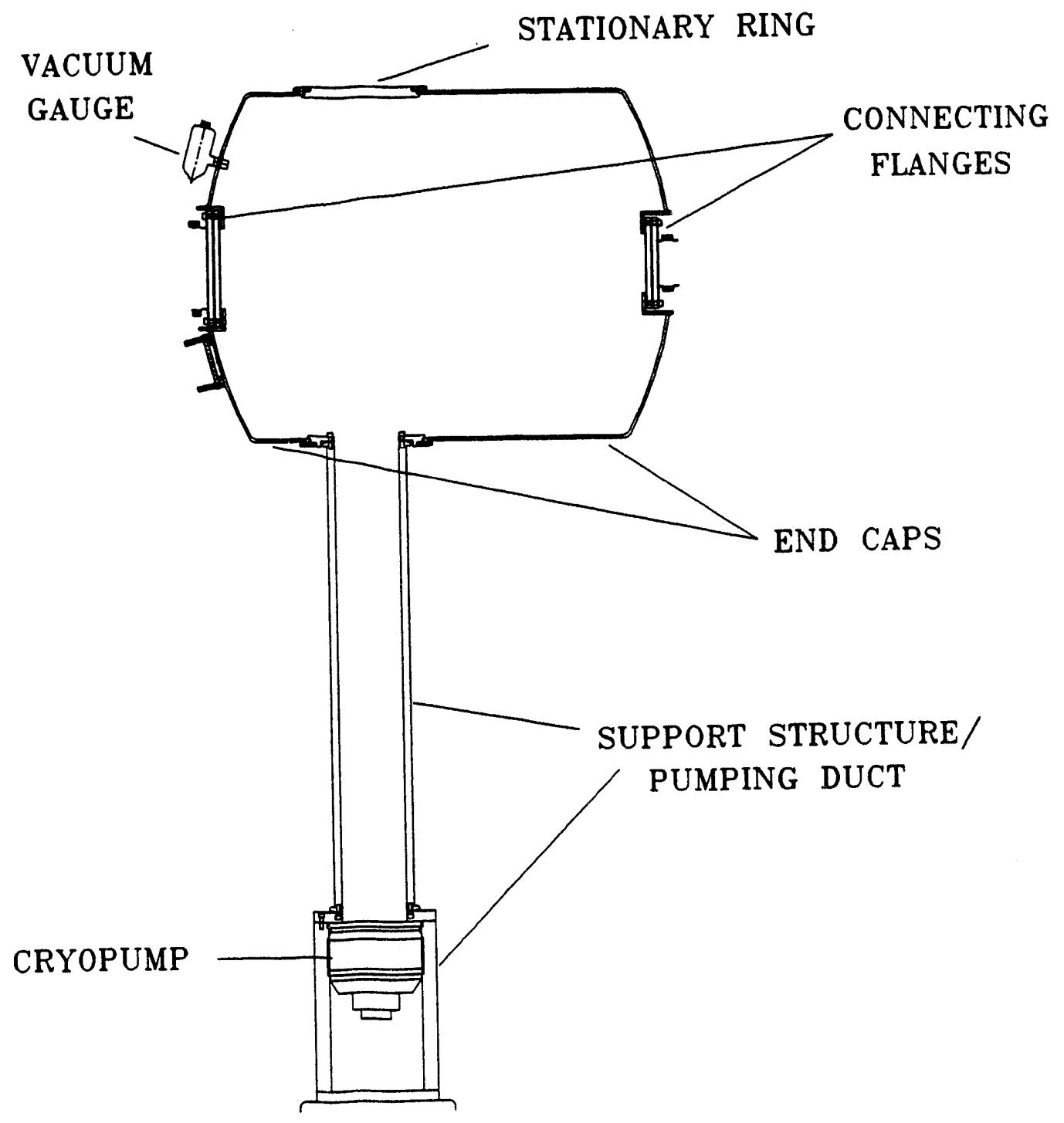

Figure 3 


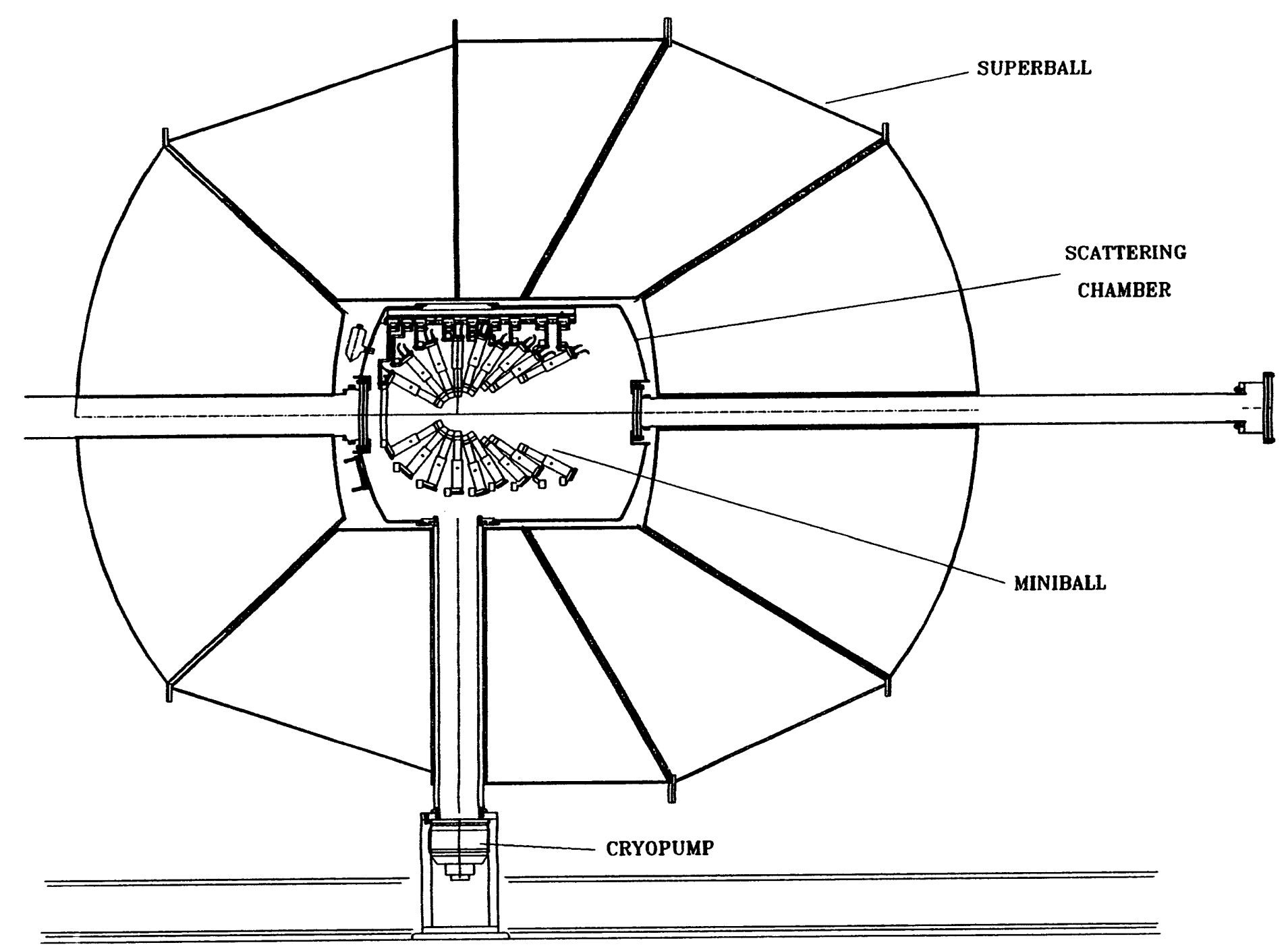




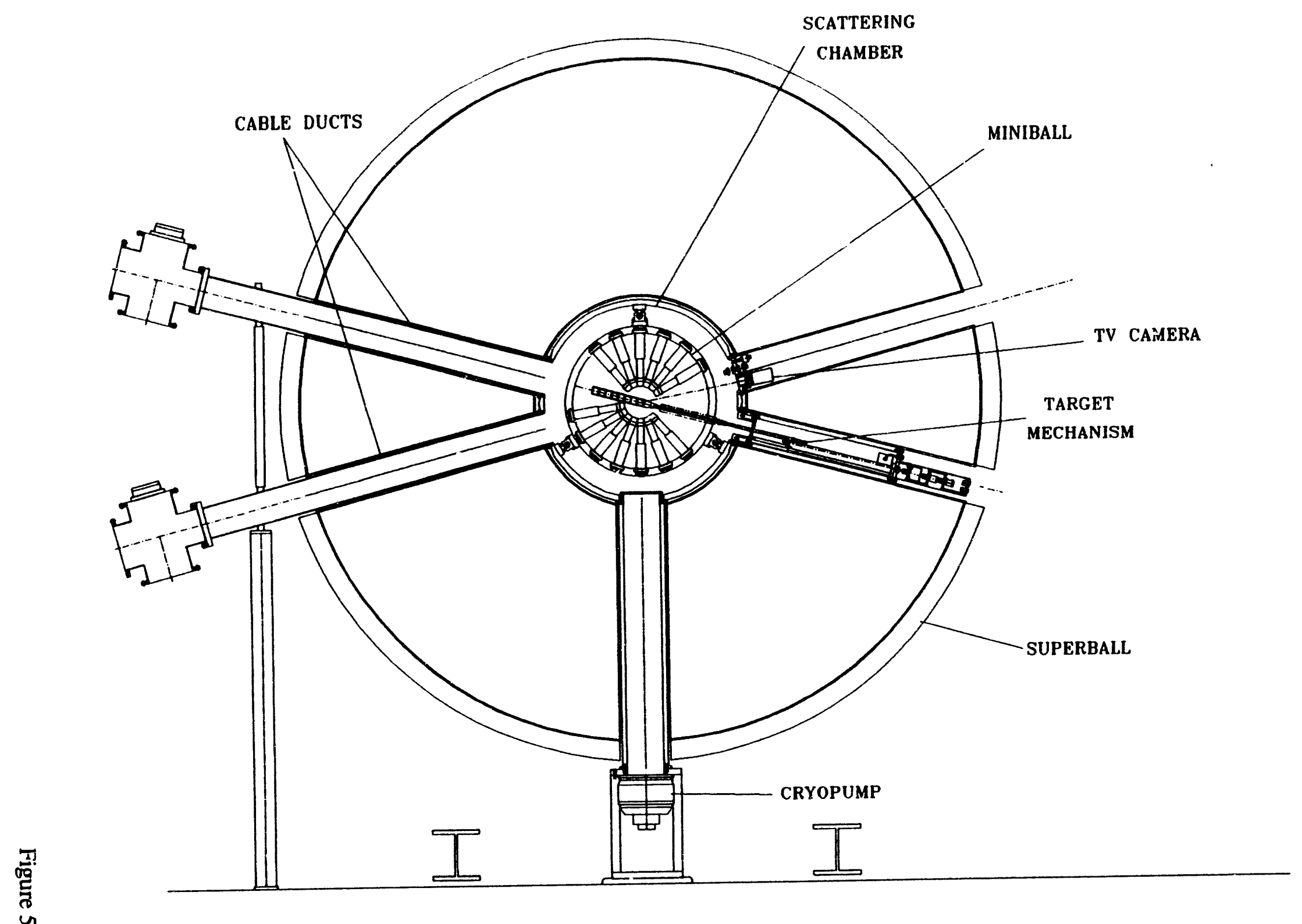




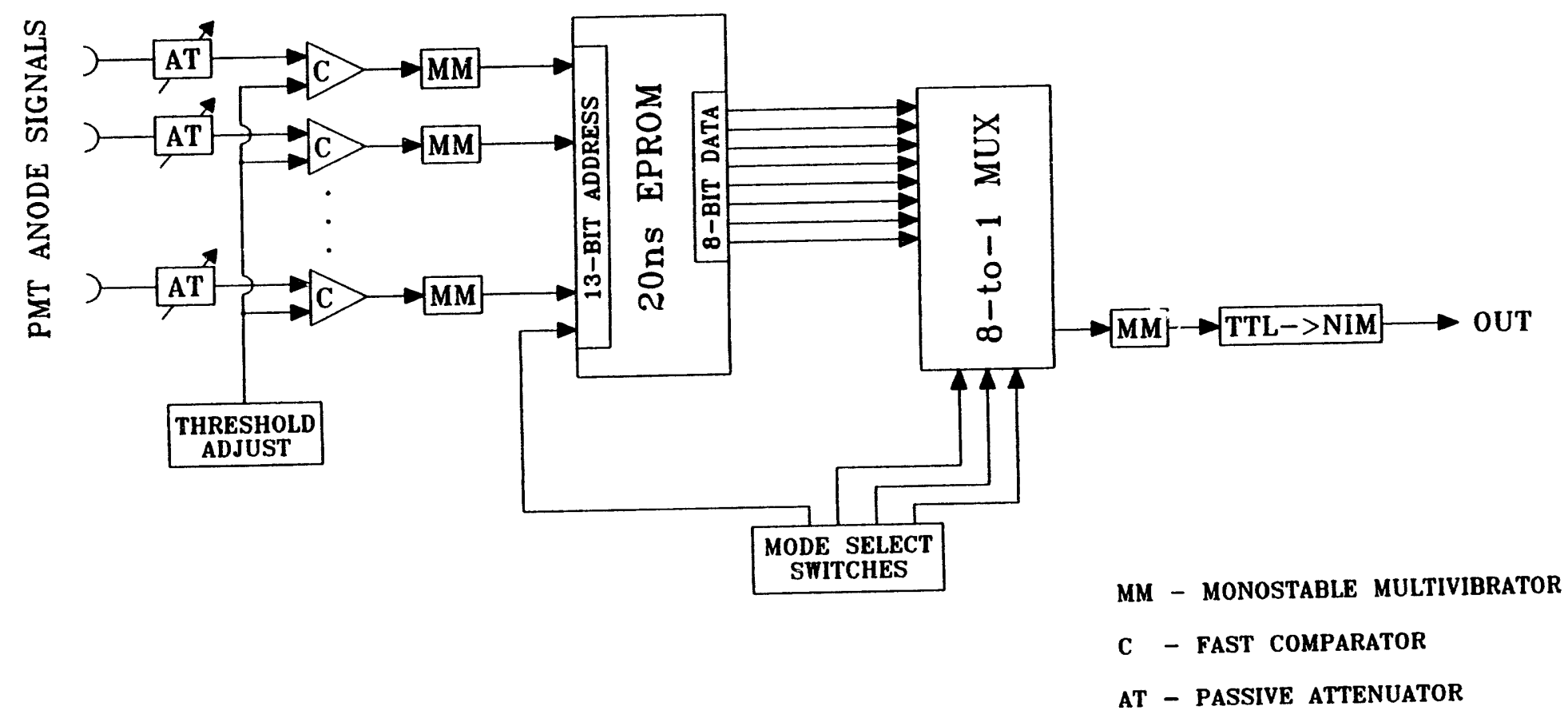




\title{
A Computer Code for Light Detection Efficiency Calculations of Photomultiplier Tubes

\author{
in Neutron Detector
}

\author{
X. Zhao, S. P. Baldwin, W. U. Schröder, and J.Töke \\ Department of Chemistry and Nuclear Structure Research Laboratory, \\ University of Rochester, Rochester, N.Y. 14627
}

(June 30, 1993)

\begin{abstract}
A computer code for calculating the light detection efficiency of the photomultiplier array of a $4 \pi$ neutron detector was written, for a wide class of complex detector shapes. Structure and applications of the code are discussed, and some simulation results are presented. The trade-off between light collection efficiency and sensitivity of the detector to the position of neutron interaction sites in the scintillator are discussed.
\end{abstract}

\section{INTRODUCTION}

In studies of intermediate-energy heavy-ion reactions, neutron detectors play more and more important roles [1-3]. To detect neutrons efficiently, not only a high probability for neutron capture, depending on size and shape of the detector and its scintillator [4]; but also high efficiency for light collection by the photomultiplier (PMT) are needed.

In experiments, neutron detectors (Neutron Multiplicity Meters) are used to measure the multiplicity and total kinetic energy of neutrons emitted in a reaction event $[5,6]$. The operational principle of a liquid scintillator neutron detector $[7,8]$ is as follows: after a neutron enters the detector tank, it undergoes first a moderation process due to scattering off hydrogen and carbon nuclei in the scintillator. After having been slowed down to thermal energy, the neutron has a high probability to be captured by gadolinium nuclei in the scintillator. Neutron capture in Gd is followed by a cascade of $\boldsymbol{\gamma}$-rays. The $\boldsymbol{\gamma}$-rays interact with electrons 
of the scintillator mainly by Compton scattering, and finally, the recoil electrons generate scintillation light (photons), which is detected by the photomultipliers of the detector.

In order to evaluate the responses of the neutron detector to different nuclear reaction scenarios and in different experiment setups, it is important to perform computer simulations. There already exist computer codes for the calculation of neutron detection (capture) efficiency, such as the code DENIS and its modified versions $[9,10]$. These codes simulate the neutron capture process and $\boldsymbol{\gamma}$-ray scattering processes rather well [4], but does not treat the light collection processes in sufficient detail.

The efficiency of light collection depends on several factors: the number of photomultiplier tubes in the detector; the reflection coefficient of the interior walls of the detector which may or may not be coated with reflective material; and the light attenuation length. The effects of coating on the light collection represent important aspects to be considered in the design of a neutron detector. It is also of practical interest to estimate the azimuth-angle angular resolution of the neutron detectors and its dependence on the efficiency of the light collection.

For these purposes, the general FORTRAN program DELPT (Detection Efficiency of Light by Photomultiplier-Tubes) was developed, it can simulate the light collection efficiency for a large variety of detector geometries and PMT arrangements.

\section{CODE DELPT}

The code DELPT is built of a number of routines; with the main routine controlling input, output, and communication between subroutines. Monte Carlo calculations are performed for events, defined essentially by the photon emission process, i.e., the numbers of the emitted photons in the stopping of the recoiling Compton electrons. Each event produces a certain number of photons in random directions from the origin of the event, the event energy is defined as the emitted light energy. A photon travels within one of the tanks of the neutron detectors until it hits the wall of the tank or a window part carrying a PMT. In the latter case, the photon is assumed to be collected by the PMT. Otherwise it can be reflected from the wall with a certain probability if the interior of the tank is coated with reflective material, or ends its history. The probability for reflection of a photon rather than its absorption is calculated from the reflection coefficient, and a new propagation direction is generated for the photon determining the next intersection point. The probability for a photon to reach this point is given by attenuation length and the distance to be traveled by the photon in the scintillator. The above process is repeated again and again, until either the photon is collected by a PMT, or its surviving probability is below a given cutoff value. For a single event, the probabilities of all photons collected by a PMT will be summed up, it is essentially the detected photon multiplicity of the event. The detected photon multiplicity, along with the energy of each photon, will give the measured energy of the event. One defines an event as detectable if the measured event energy exceeds a given energy threshold. Finally, 
summing over the measured energies of the detectable events and comparing the results with the total light energy of the all initial events, one gets the light collection efficiencies of each PMT.

Subroutine GEOMETRY is a modified version of the existing GEOM subroutine [10], constructing the trajectory of a photon relative to the boundaries of the detector tanks. The geometry of the neutron detector allowed by the code can be quite complicated. For example, the whole detector may consist of several separate detectors, as in the case of the SuperBall. The GEOMETRY subroutine handles this problem by constructing each single detector from basic elements, spheres, planes, tubes, cones and cylinders. The GEOMETRY subroutine calculates the intersection of a photon trajectory with the walls of these basic elements, it returns the coordinates of the intersection points, which are used to determine the propagation direction of the reflected photon. Actually, the GEOMETRY subroutine calls several other existing subroutines to do the detailed calculations for each basic element [10].

The subroutine NOR_DIR uses the coordinates of the point of intersection of the photon trajectory with the inside wall of the corresponding detector element to determine the normal vector of the reflection plane.

Fig 1 shows the symbolic flowchart for the code DELPT. The "initialization" block clears the arrays which store the output data, it also calculates the coordinates of all PMT. In the figure, RAN.DIR is a subroutine called by the main routine, generating a random propagation direction for the current photon; PERF_DIR is also a subroutine called by the main routine, generating a propagation direction for the current photon after a perfect reflection at the detector wall. The condition "end of photon history?" is satisfied when either the photon intersects a PMT, or its probability is below a given cutoff value. The condition "good direction?" applies only to the case of diffuse reflection and is satisfied when the photon propagation direction is towards the inside of the tank.

\section{A. Input of the code}

The input of the code consists of three parts.

The first part of the input consists of miscellaneous pararneters. These parameters are: a large integer as the seed of random number generator; the number of photons emitted per event; the initial light energy per event; the PMT threshold; the detection probability cutoff of photon by PMT; the light attenuation length of photon in the scintillator; the reflection coefficient and whether the reflection is perfect reflection or diffuse reflection.

The second part is the geometrical setup of the whole neutron detector and the corresponding photomultiplier tubes. One needs to give the total number of separate detectors first. Each single detector is constructed by the following basic elements: sphere, plane, tube, cone and cylinder. One needs to give the total number of elements in a single detector and input the parameters for each elements. The first input parameter for element is the 
name of the element; the second input parameter for element is "yes" if the scintillator is inside the element or "no" if the scintillator is outside the element, except for plane. Then, for "sphere", one needs to input the radius and the coordinates $(X, Y, Z)$ of the center; for "plane", one needs to input the coordinates of a 1st. point in the plane and a 2nd. point outside the plane, the vector from the 1st. point to the 2 nd. point should be perpendicular to the plane and towards the scintillator; for "tube", one needs to input the radius and the coordinates of any two point on the axis; for "cone", one needs to input the value of the half-opening angle and the coordinates of the vertex and point which is on the axis and inside the cone; for "tube", one needs to input the radius and the coordinates of two points which are on the two end surfaces and also on the axis. In the current DELPT code, each single detector can only have one set of PMT which are arranged in a fashion symmetric about the $\mathrm{z}$ axis (symmetric axis) for each detector. For each single detector, one needs to input the total number of the PMT, the radius of the PMT, the distance from the PMT to the symmetric axis ( $z$ axis) and the $z$ coordinate of the PMT.

Finally, an input event data file consisting of event position parameters (coordinates of events) has to be defined. A typical input file is shown by the upper part of the Table 1. The file "eve.inp.dat" is a data file which stores the event coordinates for the simulation, an example of part of such data file is shown by the lower part of the Table 1.

\section{B. Output}

The code outputs, for each single neutron detector, the total number of events detected, the detection efficiency as well as the light energy detection efficiency. The code also outputs, for each PMT in the detector, the number of events detected, the detection efficiency, and the light energy detection efficiency.

The output is stored in file DE_OUT.DAT. A typical output file is shown by Table 2. The output file consists of two parts: parameters from the input, and the calculation results.

The first part basically repeats the input file of the code. The "Miscellaneous parameters" consist of the name of the input event data file, numbers of photons emitted per event, the initial total light energy per event, the threshold of PMT, and the detection probability cutoff of photon by PMT. The "some properties of the detector" include the light attenuation length of the scintillator and the reflection coefficient assumed for the interior walls of the detector, if the reflection coefficient is not zero, it will also give the the reflection type (perfect or diffuse). The information on the detector geometry includes the number of separate tank elements the whole neutron detector has, and for each separate detector element, the parameters of the basic geometrical elements and the PMT.

The second part outputs, for each detector element, the total number of events occurred in this element, the number of detected events, the corresponding event number detection efficiency as well as the energy detection efficiency. The corresponding values are given both for each of the PMT separately and for the detector element as a whole 


\section{Test}

After carefully debugging the program, several test runs were performed to check the correctness of the code.

First, a data file is generated for a spherical detector element with a radius $150 \mathrm{~cm}$, containing 5000 events all originated at the center of the sphere. The interior of the detector is not coated (reflection coefficient R_COE $=0$ ), and the detector has 12 PMT. The results are shown in Table 2. As expected all PMT have almost the same efficiency. The 12 PMT cover $0.653 \%$ of $4 \pi$ in solid angle. The probability for a photon to survive the attenuation experienced in traversing the scintillator and arrive at the boundary of the detector is calculated to be equal to $67.72 \%$. Therefore, the total energy efficiency should be about $0.45 \%$, in agreement with the numerical result predicted by the code.

Secondly, the code was tested assuming a cylindrical detector geometry. This detector is $80 \mathrm{~cm}$ long, with an outer radius of $150 \mathrm{~cm}$ and an inner radius of $50 \mathrm{~cm}$. The interior of the detector is assumed to be coated with a diffuse reflective material having reflection coefficient of $R_{-} C O E=1.0$. The detector has $12 \mathrm{PMT}$ which are equally spaced $\mathrm{a}^{+}$the outer shell of the detector, with their axes perpendicular to the axis of the cylinder. For the purpose of this test, the light attenuation length was set to a very large value $(40000 \mathrm{~cm})$. For the input event data file, 2000 randomly distributed events are generated for each of three coaxial cylindrical layers of scintillator with radius of 75,100 , and $125 \mathrm{~cm}$ respectively. The events were generated with PHI-angle randomly distributed between -15 and 15 degrees relative to PMT 6 (PMT No.6 had zero PHI value). The DELPT code was modified slightly by changing the termination condition of a photon history from one based on the survived probability to one testing the number of reflecting at the wall of the detector, with a cutoff value was set to 40 reflection times. The results of these test calculations are shown in Table 3. As expected, all events can be detected by any PMT, and the energy detection efficiency has a peak for PMT No.6. Since each PMT covers only $0.054 \%$ of $4 \pi$ in solid angle measured from the center of the detector, and the cutoff value for reflection times is 40 , one gets that each PMT has a probability of about $2 \%$ to detect an event originated at the center of the detector. So the simulation results of the efficiency of each PMT is reasonable.

\section{THE EFFECTS OF REFLECTIVE COATING ON LIGHT COLLECTION}

\section{AND PHI-ANGLE RESOLUTION}

To evaluate the effect of coating of the interior walls of a detector element with a reflective material on the light collection of PMTs and the PHI-angle resolution, the DELPT code was used to simulate the light travelling and collecting processes. The detector is essentially the same as the middle segment of the SuperBall [4]. It is a cylindrical detector element, $80 \mathrm{~cm}$ long, with an outer radius of $150 \mathrm{~cm}$ and an inner radius of $50 \mathrm{~cm}$. The detector has $12 \mathrm{PMT}$ which are equally spaced at the outer shell of the de ${ }^{2}$ ctor, with their axes perpendicular 
to the axis of the cylinder. The light attenuation length is $400 \mathrm{~cm}$, as is typical for a liquid scintillator of the kind to be used in the SuperBall. The radius of the PMT $(7 \mathrm{~cm})$, is the same as that of the Thorn EMI 9390KB fast photomultiplier tubes which will be used in the SuperBall. The event data file contains 2000 events for each of the three coaxial layers of scintillator with radii of 75,100 , and $125 \mathrm{~cm}$, the events are generated with PHI-angle randomly distributed between -15 and 15 degrees relative to PMT 6 (PMT No.6 had zero PHI value).

For the above event data file and the above detector geometries, the DELPT code was run for PMT thresholds of THR $=0.01,0.001$, and $0.0001 \mathrm{MeV}$, and for reflection coefficients of R_COE $=0,0.5,0.7$, and 1.0, where R_COE $=0$ applies essentially for an interior which is not coated. For coated interiors, the reflection type was chosen to be diffuse reflection, since this kind of reflection will be used in the SuperBall.

Fig 2, Fig 3 and Fig 4 show the numbers of the detected events by each PMT, versus the PMT numbers. Fig 2 Fig 3 and Fig 4 refer to a PMT threshold of $0.01 \mathrm{MeV}, 0.001 \mathrm{MeV}$ and $0.0001 \mathrm{MeV}$, respectively. The four panels in each of these three figures refer to four different values assumed for the reflection coefficients. It is observed from these figures that the spectra are strongly peaked at PMT No.6, for small reflection coefficients or large PMT thresholds, and that the spectra become broader for larger reflection coefficients or smaller PMT thresholds. For the bottom right panel of Fig 4, all the PMT see the same number of events (the total number of events occurred in the detector). It is clear that if one uses the timing signal to count the neutron multiplicity, the PHI-angle resolution will decrease with decreasing PMT threshold or increasing reflection coefficient.

In Figs 5, 6 and 7, the light collection efficiencies of the PMT are plotted versus the PMT number, for PMT thresholds of $0.01 \mathrm{MeV}, 0.001 \mathrm{MeV}$ and $0.0001 \mathrm{MeV}$, respectively. The four panels in each of these three figures refer to four different values of the reflection coefficients. Comparing with the neutron multiplicity measurement, it is clear that the energy efficiency spectra always have relatively well defined peaks at PMT No.6, for all reflection coefficients and PMT thresholds. PMT No.6 accounts for about $50 \%$ of the total light energy collection. Therefore the energy measurement is predicted to give always a fairly good PHI-angle resolution, which does not depend very much on PMT threshold and reflection coefficient.

Fig 8 shows dependence of the light collection efficiency on the value of the reflection coefficient for events generated in a scintillator layer at $R=100 \mathrm{~cm}$ and for different PMT thresholds. Obviously, the light collection efficiency increases with increasing magnitude of the reflection coefficient. For relatively low reflectivities, the light collection efficiency increases relatively slowly with increasing magnitude of the reflection coefficient. This increase is much more dramatic for the higher reflectivities. This is because the non-zero value of the probability cutoff does not allow simulations with different reflection coefficients to have the same average reflection times, simulations with higher different reflection coefficients will have larger average reflection times.

From the results of these simulations, one can conclude that a detector with a highly 
reflective interior will have a much better light collection efficiency. At the same time, one can also get a fairly good PHI-angle resolution using the energy signals, without sacrifice in absolute detection efficiency.

\section{FUTURE MODIFICATIONS OF THE CODE}

For the DELPT code, some modifications can be made as required by the specific future applications. a) For a complicated geometrical setup of PMT, the input parameters could include the coordinates of each individual PMT. b) In the input event data file, one could include initial energy of each event for the sake of using simulation results of other codes, such as DENIS, giving the continuation of the efficiency simulations [4], accordingly, the photon number of each event in the input file should be replaced by the photon energy. Since the event energy output from DENIS is the kinetic energy of the recoil electron, and the conversion from recoil energy of electron to emitted light energy is not $100 \%$, one should also include the quantum efficiency of this conversion into the input and use it to get the realistic light energy of an event. c) This quantum efficiency and energy detection efficiency of PMT could be combined in the code to predict a realistic light output measured by the PMT and, hence, an overall energy resolution.

\section{SUMMARY}

A computer code has been written for calculating light detection efficiency of photomultiplier tubes in a liquid scintillator neutron detector. Detailed descriptions of the code as well as sample input and output files are presented. The results of the simulation show that a high reflection coefficient of a coating of the interior walls of the detector will increase the light collection efficiency but still afford a fairly good PHI-angle resolution if the energy signals are used for this latter purpose.

This work was supported by the U.S. Department of Energy under Grant No. DE-FG0288ER40414 and by the U.S. Department of Energy University Research Instrumentation Grant No. DE-FG05-91ER79048. 


\section{REFERENCES}

[1] W.U. Schröder Nucl. Phys. A538, 439c (1992), and ref. therein.

[2] U. Jahnke et al., Proc. XX International Summer School Nucl. Phys., Mikolajki, 1988, Report HMI-B 463, 1988

[3] J. Galin, Proc. 1st Europ. Worksh. Nucl. Phys., Megève, 1991, World Scientific (Singapore 1991), p.23

[4] X. Zhao et al., Report DOE/ER/40414-5, p.190 (1992); unpublished

[5] B. Lott et al., Phys. Rev. Lett. 68, 314 (1992)

[6] S.P. Baldwin et al., Proceedings of the 9th Winter Workshop on Nuclear Dynamics, Key West, 1993, in press

[7] B.C. Diven, H.C. Martin, R.F. Taschek, Terrell, Phys. Rev. 101, 1012 (1956)

[8] U. Jahnke et al., Lect. Notes in Physics 178, 179 (1983)

[9] J. Poitou and C. Signarbieux, Nucl. Inst. Meth. 114, 113 (1974)

[10] B.M. Quednau et al., Report DOE/ER/40414-2, p.170 (1989); unpublished 


\section{FIGURES}

FIG. 1. the symbolic flowchart of the main routine of the code DELPT. the functions of the subroutines GEOMETRY, NOR_DIR, RAN_DIR and PERF_DIR are discussed in Sec.3

FIG. 2. detected event number by PMT vs. PMT index. the initial event number is 2000 . the initial energy of event is $1 \mathrm{MeV}$ and the threshold of the PMT is $0.01 \mathrm{MeV}$.

FIG. 3. detected event number by PMT vs. PMT index. the initial event number is 2000. the initial energy of event is $1 \mathrm{MeV}$ and the threshold of the PMT is $0.001 \mathrm{MeV}$.

FIG. 4. detected event number by PMT vs. PMT index. the initial event number is 2000. the initial energy of event is $1 \mathrm{MeV}$ and the threshold of the PMT is $0.0001 \mathrm{MeV}$.

FIG. 5. light collection efficiency of PMT vs. PMT index. the initial energy of event is $1 \mathrm{MeV}$ and the threshold of the PMT is $0.01 \mathrm{MeV}$.

FIG. 6. light collection efficiency of PMT vs. PMT index. the initial energy of event is $1 \mathrm{MeV}$ and the threshold of the PMT is $0.001 \mathrm{MeV}$.

FIG. 7. light collection efficiency of PMT vs. PMT index. the initial energy of event is $1 \mathrm{MeV}$ and the threshold of the PMT is $0.0001 \mathrm{MeV}$.

FIG. 8. light collection efficiency vs. reflection coefficient. the initial energy of event is $1 \mathrm{MeV}$ and the threshold of the PMT is $0.001 \mathrm{MeV}$. 


\section{Table 1}

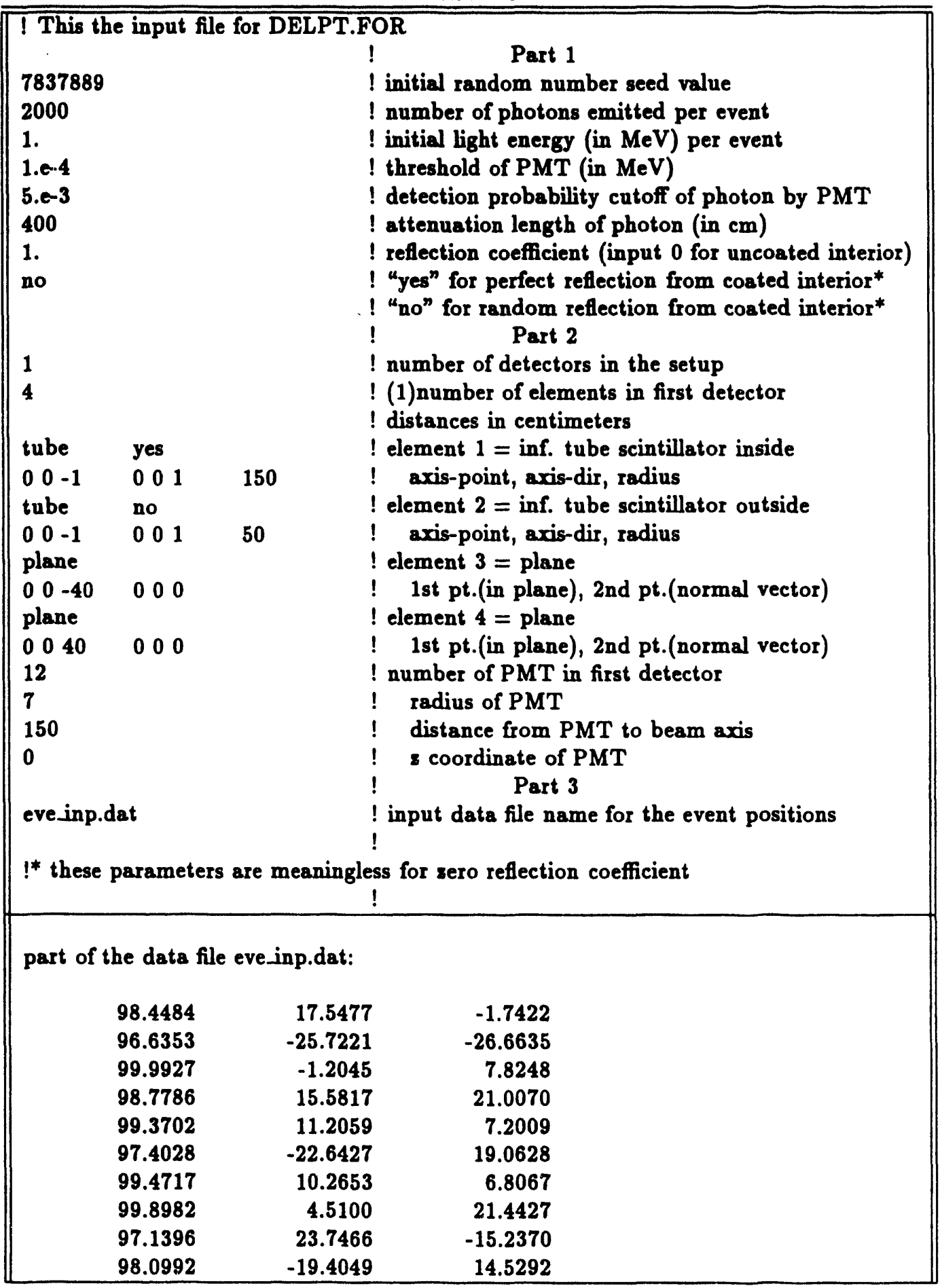


Table 2

\begin{tabular}{|c|c|c|c|}
\hline \multicolumn{4}{|c|}{ ************* OUTPUT OF PROGRAM DELPT $* * * * * * * * * * * * *$} \\
\hline \multicolumn{4}{|c|}{$\begin{array}{l}\text { Miscellaneous parameters } \\
\text { (1) Input event (positions) data file name = eve.inp.dat } \\
\text { (2) Photon number per event }=1000 \\
\text { (3) Initial light energy per event }=1.0 \mathrm{MeV} \\
\text { (4) Threshold of PMT }=0.50 \mathrm{E}-03 \mathrm{MeV} \\
\text { (5) Detection probability cutoff of photon by } \mathrm{PMT}=0.10 \mathrm{E}-01\end{array}$} \\
\hline \multicolumn{4}{|c|}{$\begin{array}{l}\text { Some properties of the detector } \\
\text { (1) The reflection coefficient }=0.0 \\
\text { (2) Attenuation length }=400.0 \mathrm{~cm}\end{array}$} \\
\hline \multicolumn{4}{|c|}{$\begin{array}{l}\text { Detector geometry : } \\
\text { Number of defined detectors } 1 \\
\text { detector } 1 \text { is defined by } 1 \text { elements } \\
\text { element } 1 \text { is sphere } \\
\text { center point }(0.00,0.00,0.00) \\
\text { radius } 150.00 \mathrm{~cm} \\
\text { detector } 1 \text { has } 12 \text { PMT } \\
\text { radius of the PMT } 7.0 \mathrm{~cm} \\
\text { distance from PMT to beam axis } 150.00 \mathrm{~cm} \\
\text { z coordinate of PMT } 0.00 \mathrm{~cm}\end{array}$} \\
\hline \multicolumn{4}{|c|}{$* * * * * * * * * * * * * * * * *$ RESULTS $* * * * * * * * * * * * * * * * * *$} \\
\hline \multicolumn{4}{|c|}{ DETECTOR 1: Total event number $=5000$} \\
\hline $\begin{array}{l}\text { PMT } \\
1\end{array}$ & $\underset{2101}{\text { ABS_NUMBER }}$ & $\begin{array}{l}\text { NUMBER_EFF(\%) } \\
42.02\end{array}$ & $\underset{0.04}{\operatorname{ENERGYEFF}(\%)}$ \\
\hline 2 & 2098 & 41.96 & 0.04 \\
\hline 3 & 2059 & 41.18 & 0.04 \\
\hline 4 & 2060 & 41.20 & 0.04 \\
\hline 5 & 2061 & 41.22 & 0.04 \\
\hline 6 & 2137 & 42.74 & 0.04 \\
\hline 7 & 2102 & 42.04 & 0.04 \\
\hline 8 & 2131 & 42.62 & 0.04 \\
\hline 9 & 2136 & 42.72 & 0.04 \\
\hline 10 & 2133 & 42.66 & 0.04 \\
\hline 11 & 2129 & 42.58 & 0.04 \\
\hline 12 & 2119 & 42.38 & 0.04 \\
\hline TOTAL & 4991 & 99.82 & 0.45 \\
\hline
\end{tabular}


Table 3

\begin{tabular}{|c|c|c|c|}
\hline \multicolumn{4}{|c|}{$* * * * * * * * * * * * * * * * *$ RESULTS OF TEST 2 2******************} \\
\hline \multicolumn{4}{|c|}{ LAYER $1:$ Radius = $75.0 \mathrm{~cm}$} \\
\hline${ }_{1}^{\text {PMT }}$ & $\begin{array}{c}\text { ABS_NUMBER } \\
2000\end{array}$ & $\begin{array}{c}\text { NUMBER_EFF(\%) } \\
100.00\end{array}$ & $\underset{1.34}{\text { ENERGYEFF(\%) }}$ \\
\hline 2 & 2000 & 100.00 & 1.45 \\
\hline 3 & 2000 & 100.00 & 1.61 \\
\hline 4 & 2000 & 100.00 & 1.83 \\
\hline 5 & 2000 & 100.00 & 2.06 \\
\hline 6 & 2000 & 100.00 & 2.23 \\
\hline 7 & 2000 & 100.00 & 2.06 \\
\hline 8 & 2000 & 100.00 & 1.82 \\
\hline 9 & 2000 & 100.00 & 1.60 \\
\hline 10 & 2000 & 100.00 & 1.45 \\
\hline 11 & 2000 & 100.00 & 1.32 \\
\hline 12 & 2000 & 100.00 & 1.28 \\
\hline TOTAL & 2000 & 100.00 & 20.05 \\
\hline \multicolumn{4}{|c|}{ LAYER $2:$ Radius $=100.0 \mathrm{~cm}$} \\
\hline $\begin{array}{l}\text { PMT } \\
1\end{array}$ & $\underset{2000}{\text { ABS_NUMBER }}$ & $\begin{array}{c}\text { NUMBER_EFF(\%) } \\
100.00\end{array}$ & $\underset{1.31}{\operatorname{ENERGYEFF}(\%)}$ \\
\hline 2 & 2000 & 100.00 & 1.45 \\
\hline 3 & 2000 & 100.00 & 1.61 \\
\hline 4 & 2000 & 100.00 & 1.79 \\
\hline 5 & 2000 & 100.00 & 2.06 \\
\hline 6 & 2000 & 100.00 & 2.39 \\
\hline 7 & 2000 & 100.00 & 2.08 \\
\hline 8 & 2000 & 100.00 & 1.78 \\
\hline 9 & 2000 & 100.00 & 1.60 \\
\hline 10 & 2000 & 100.00 & 1.46 \\
\hline 11 & 2000 & 100.00 & 1.33 \\
\hline 12 & 2000 & 100.00 & 1.29 \\
\hline TOTAL & 2000 & 100.00 & 20.14 \\
\hline \multicolumn{4}{|c|}{ LAYER $3:$ Radius $=125.0 \mathrm{~cm}$} \\
\hline $\begin{array}{l}\text { PMT } \\
1\end{array}$ & $\begin{array}{c}\text { ABS_NUMBER } \\
2000\end{array}$ & $\begin{array}{c}\text { NUMBER_EFF(\%) } \\
100.00\end{array}$ & $\begin{array}{c}\text { ENERGY EFF(\%) } \\
1.30\end{array}$ \\
\hline 2 & 2000 & 100.00 & 1.43 \\
\hline 3 & 2000 & 100.00 & 1.58 \\
\hline 4 & 2000 & 100.00 & 1.76 \\
\hline 5 & 2000 & 100.00 & 2.04 \\
\hline 6 & 2000 & 100.00 & 2.75 \\
\hline 7 & 2000 & 100.00 & 2.05 \\
\hline 8 & 2000 & 100.00 & 1.74 \\
\hline 9 & 2000 & 100.00 & 1.60 \\
\hline 10 & 2000 & 100.00 & 1.45 \\
\hline 11 & 2000 & 100.00 & 1.30 \\
\hline 12 & 2000 & 100.00 & 1.28 \\
\hline TOTAL & 2000 & 100.00 & 20.29 \\
\hline
\end{tabular}




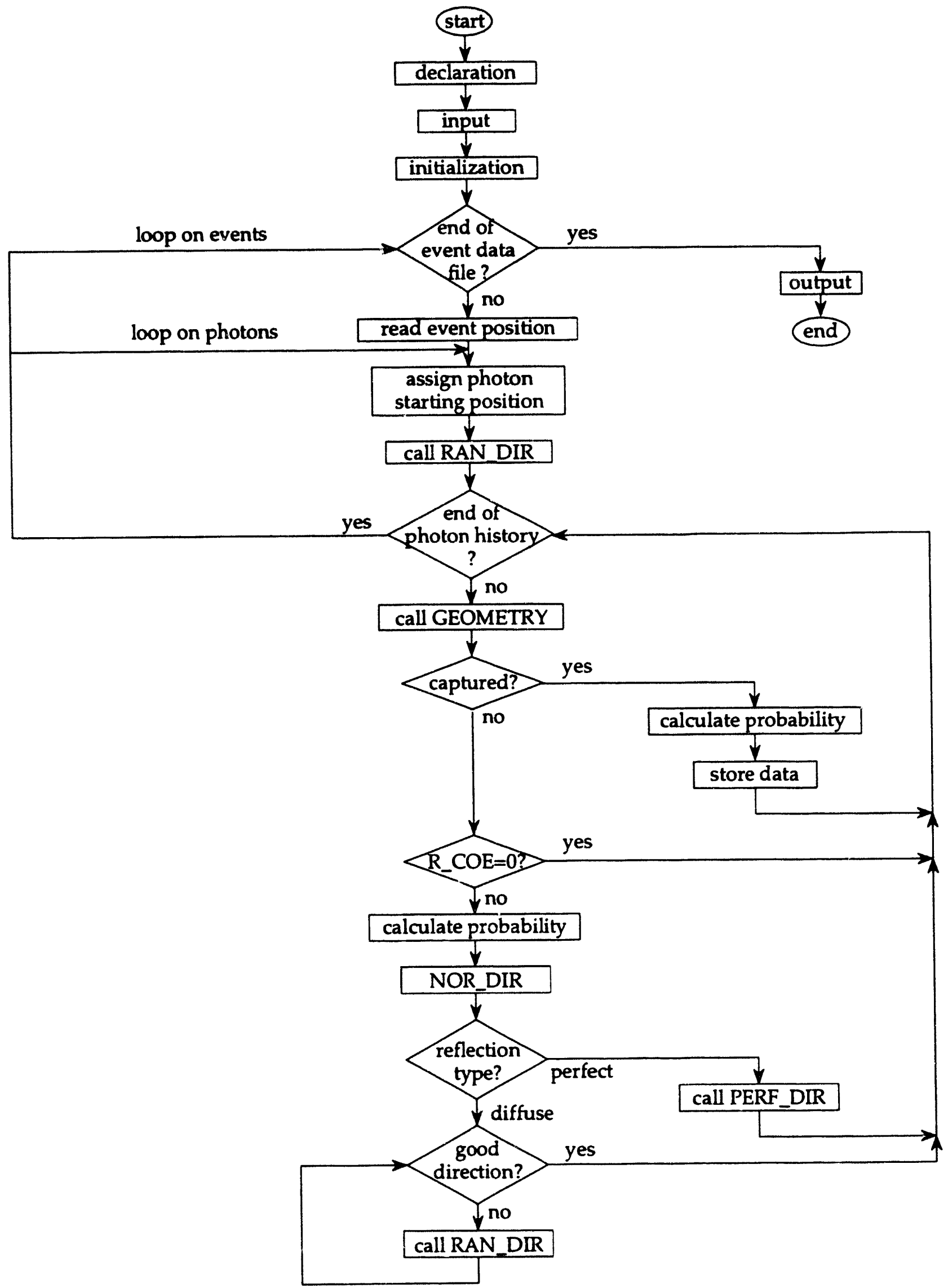

Fig. 1 


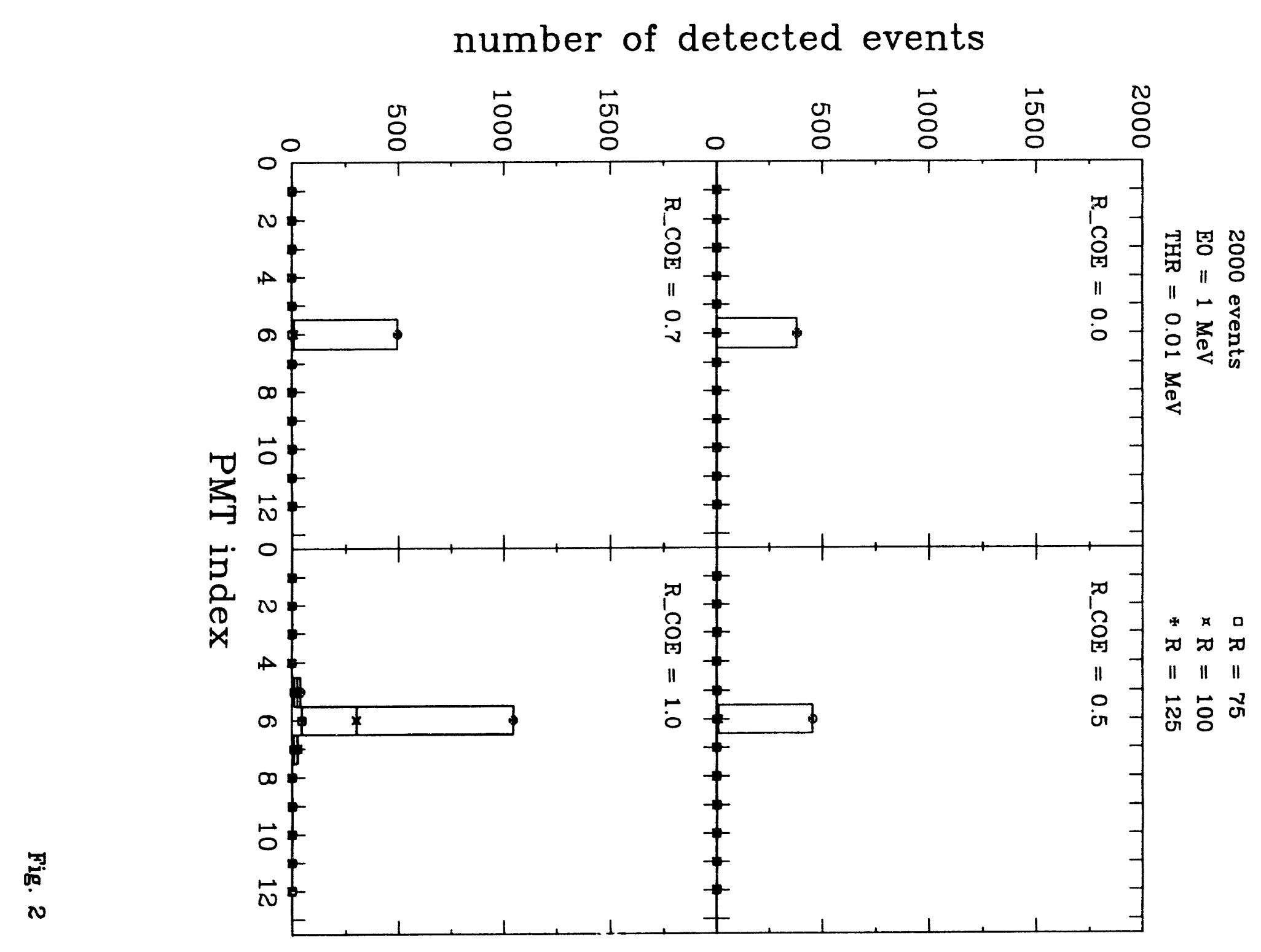




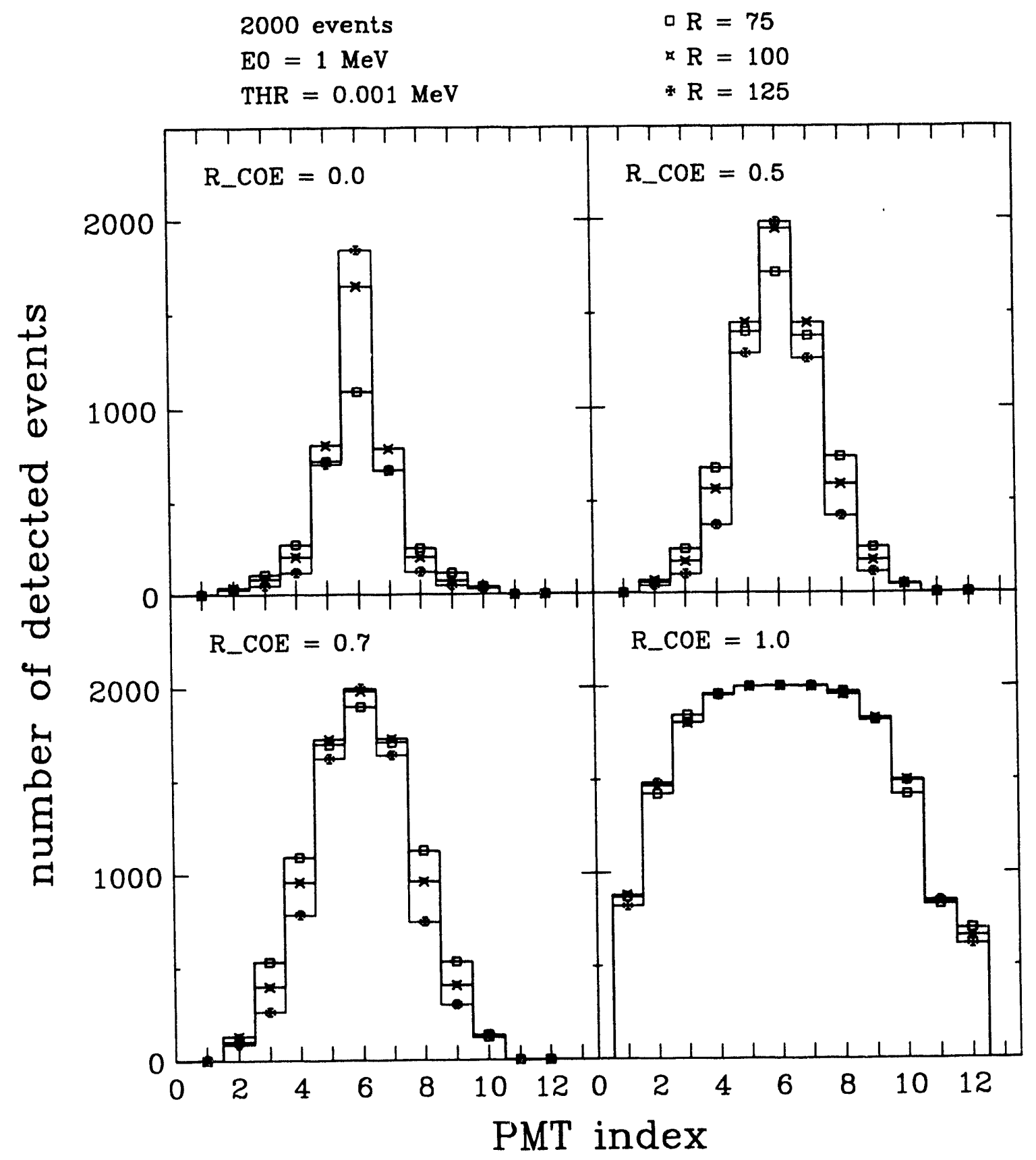

Fig. 3 


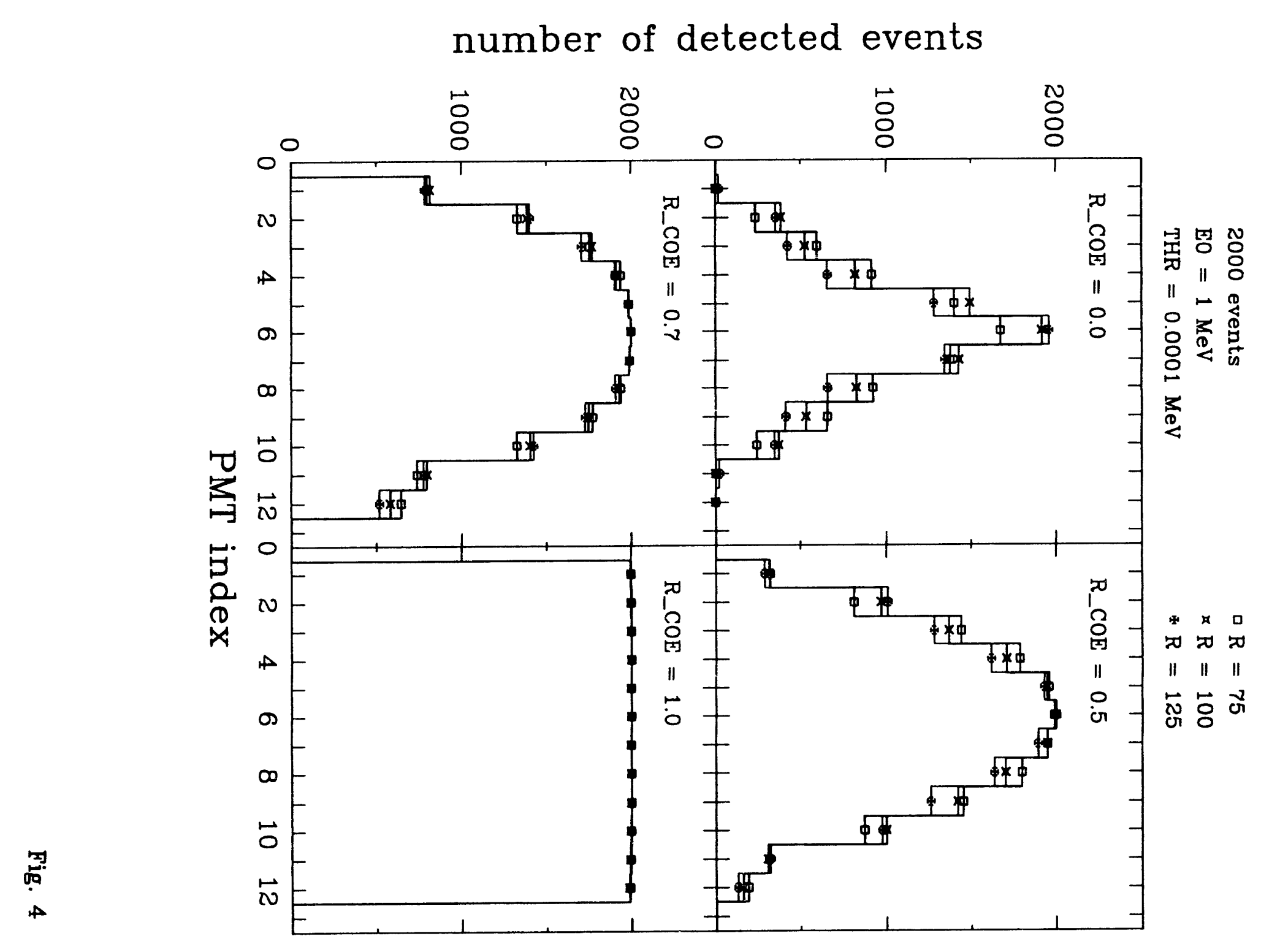




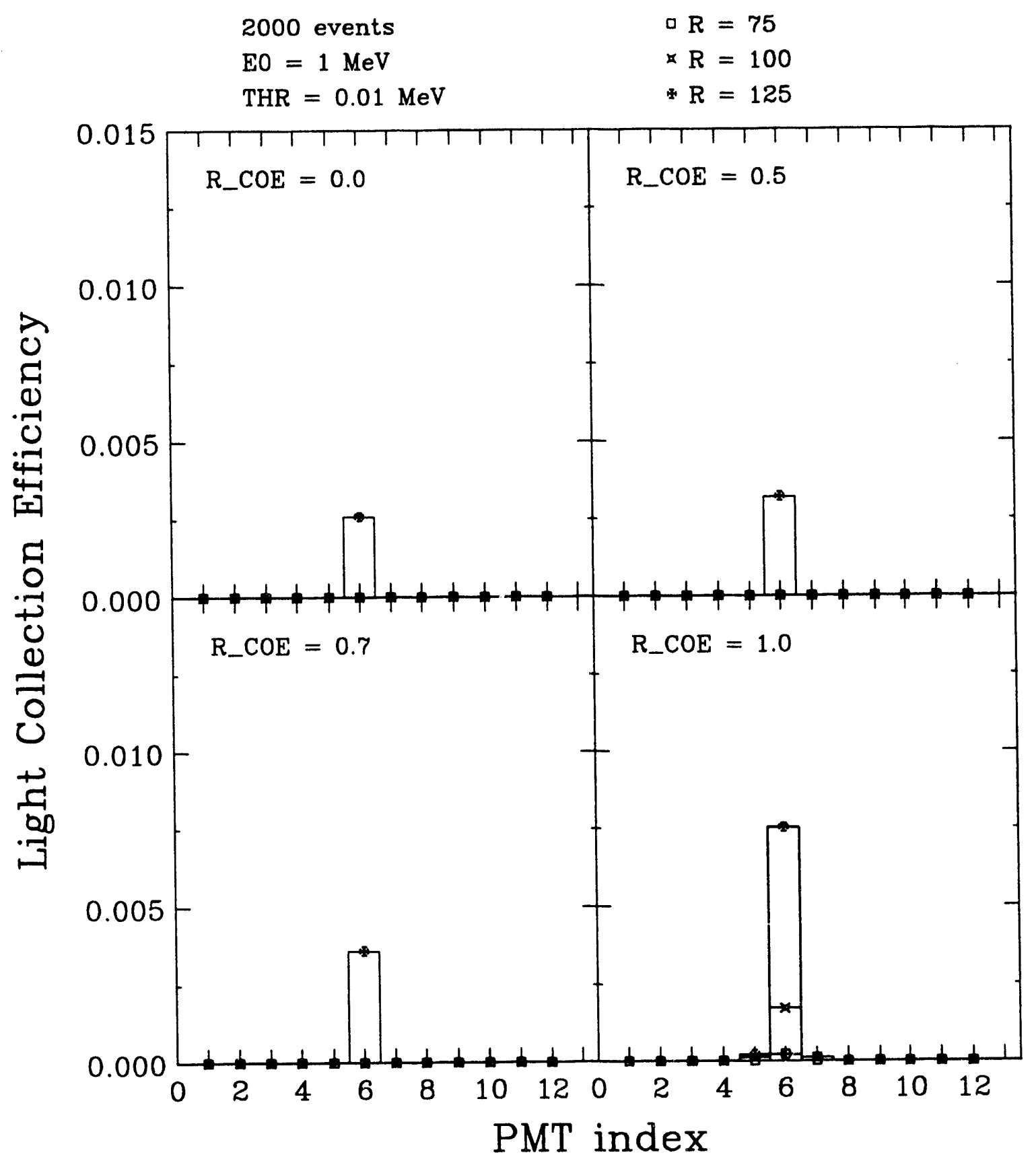

Fig. 5 


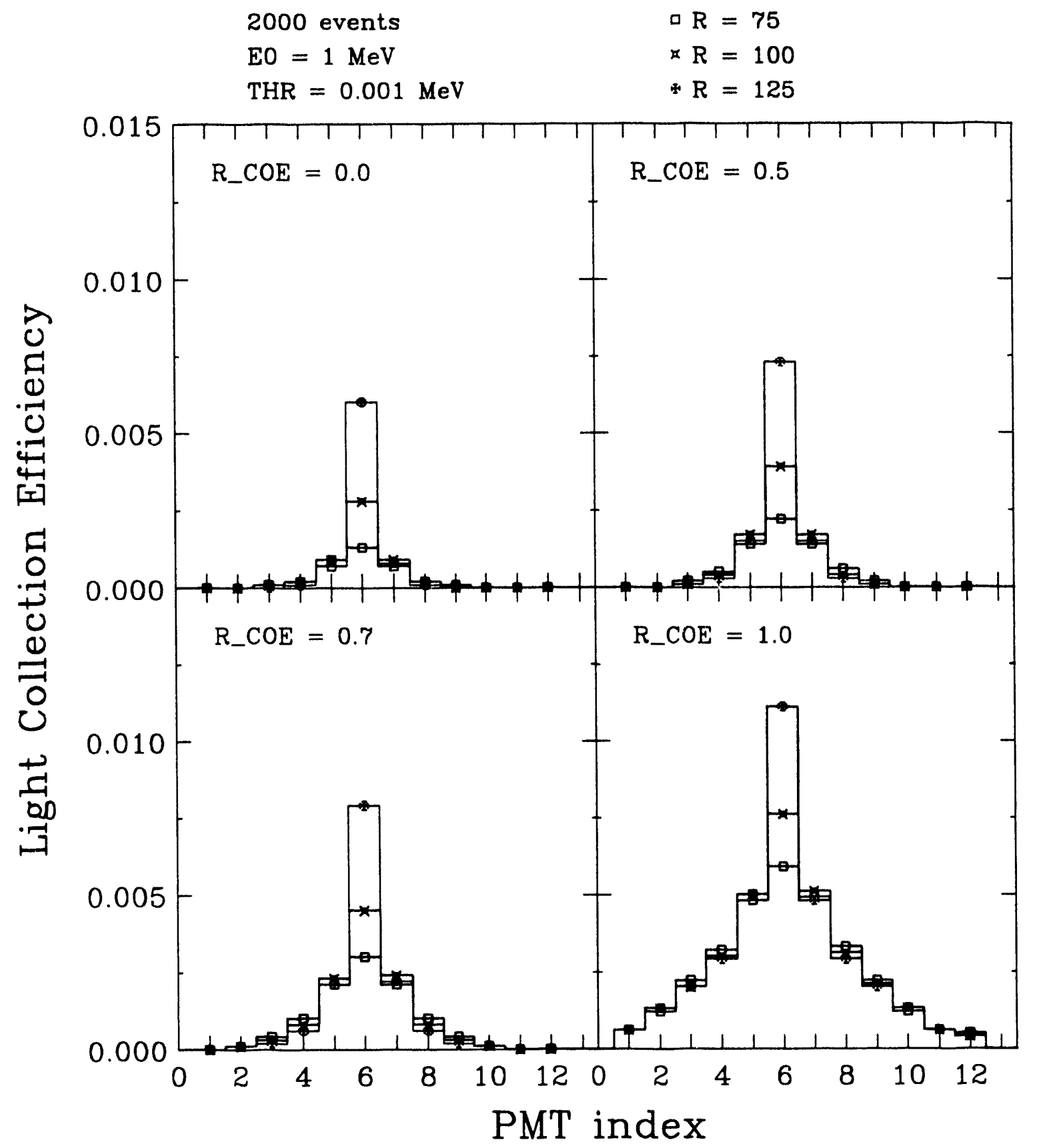

Fig. 6 


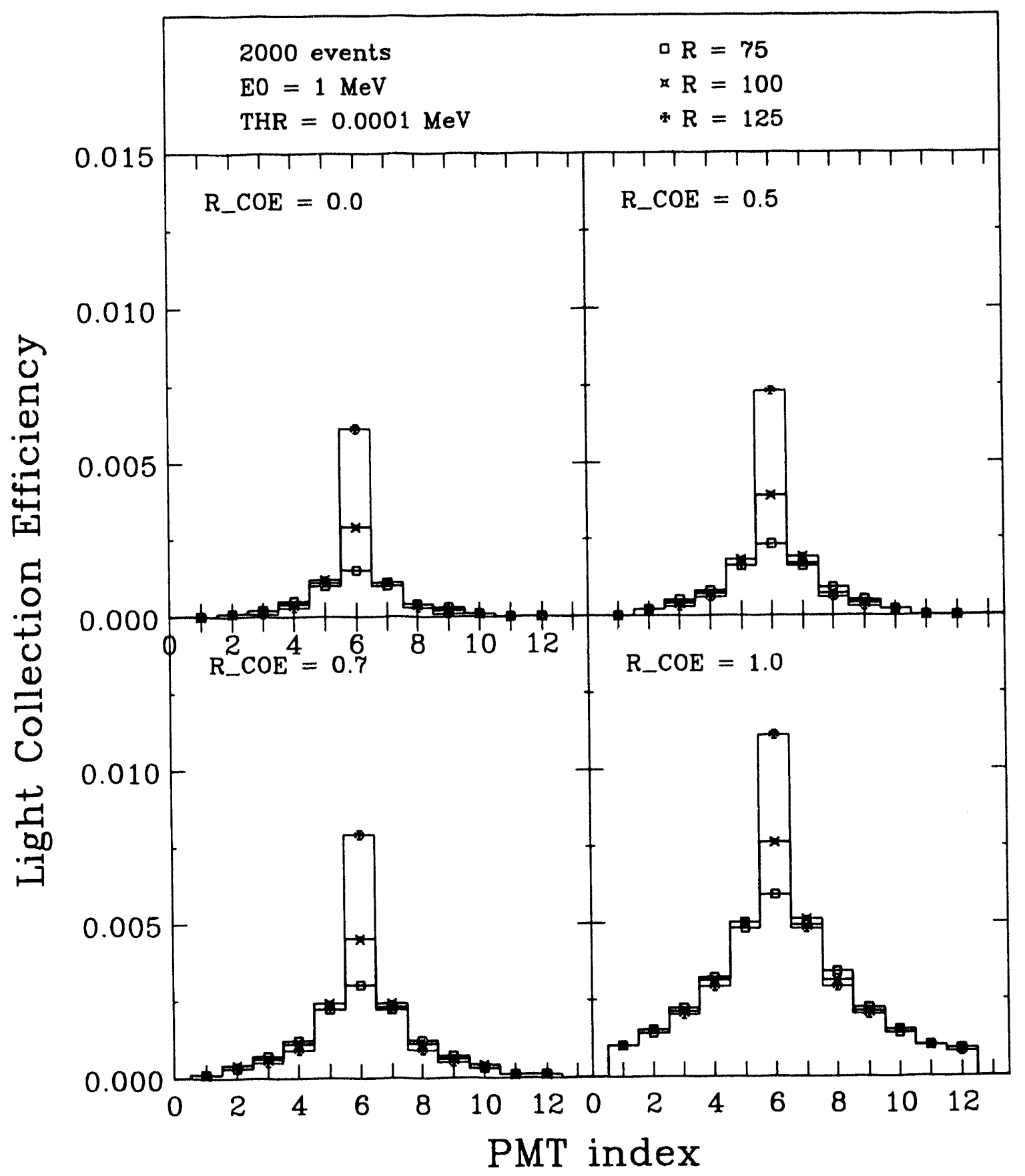

Fig. 7 


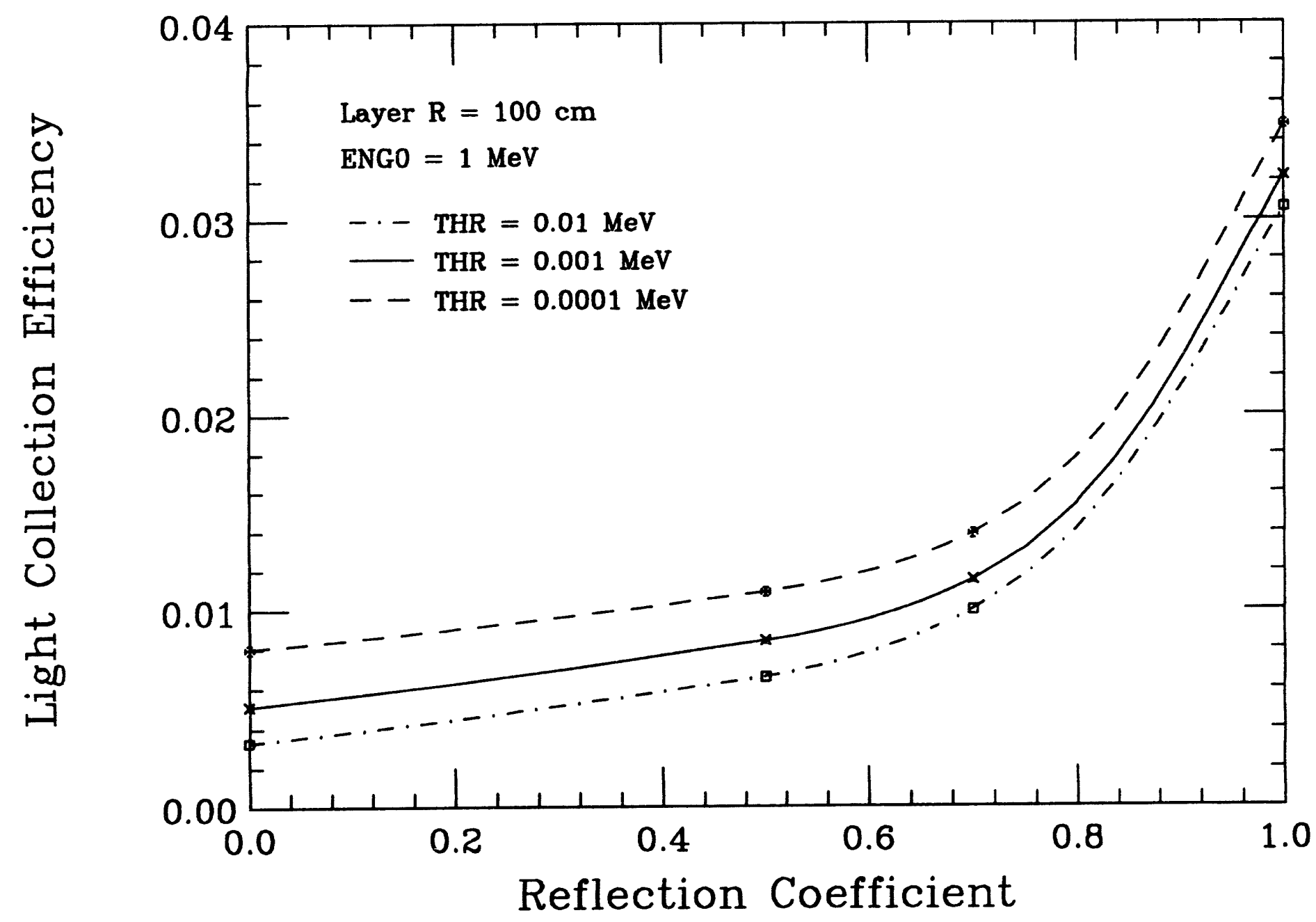




\title{
Evaluation of Gd-Loaded Liquid Scintillators for the SuperBall Neutron Calorimeter
}

\author{
S. A. Masserant, X. Zhao, S. P. Baldwin, W. U. Schröder \\ Department of Chemistry and Nuclear Structure Research Laboratory, \\ University of Rochester, Rochester, N.Y. 14627
}

\begin{abstract}
In order to choose an effective liquid scintillator for the SuperBall neutron calorimeter, currently under construction, samples of Gd-loaded organic liquid scintillator manufactured by Bicron Corporation and National Diagnostics were studied. A series of test were performed, in order to evaluate the following properties of each fluid: total light output generated for a given excitation energy, attenuation length of the emitted radiation, energy resolution, and compatibility achieved with a specified photomultiplier.
\end{abstract}

\section{INTRODUCTION}

A large, segmented $4 \pi$-detector, the SuperBall, is currently under construction at the University of Rochester. The SuperBall when filled with the proper scintillator, will serve as a neutron calorimeter. This detector measures, on an event-by-event basis, both the average kinetic energy and the multiplicity of fast neutrons emitted in a nuclear reaction. The efficiency and accuracy with which this is done, is strongly dependent on the type of scintillator used. For this reason, careful consideration should be given to the specific properties of individual scintillators, before one is chosen.

Neutron calorimeters are designed to take fast neutrons emitted from a nuclear reaction and slow them down to thermal energies through sequential elastic scattering with the scintillator constituents. This process allows neutrons to transfer some portion of their original kinetic energy to the recoil nucleus. The recoil nucleus, in turn, dissipates its energy through excitations of the luminescent scintillator. Once excited, the luminescent molecules fluoresce, emitting photons in the visible/UV region, which can be detected by a photomultiplier. The now thermalized neutrons diffuse for times on the order of $10 \mu \mathrm{s}$ through 
the scintillator tank, until they are captured by a Gd nucleus, triggering the emission of a gamma- ray cascade. Since this capture is statistically distributed over a time scale of many microseconds, the signals generated from each neutron capture can be counted on an individual basis [1].

Organic liquid scintillators offer the simplest and most economical way to accurately acquire this information in a large- volume detector. Such scintillators are composed primarily of an aromatic solvent, which is not only luminescent in nature but also rich in hydrogen $[2,3]$. The high percentage of hydrogen atoms in the fluid optimizes the moderation process since the fraction of the neutron kinetic energy transferred to the recoil nucleus through elastic scattering is maximized for equal masses of the collision partners. Moreover, this type of scintillator is easily loaded with additional material, such as wavelength shifters which can increase the compatibility of the scintillator with a particular photomultiplier.

Prior to purchasing more than 16000 liters of scintillator fluid required for the SuperBall, samples of Gd loaded liquid organic scintillator were obtained from both Bicron Corporation and National Diagnostics. A series of tests were performed, in order to determine the following characteristics of each fluid: total light output generated for a given excitation energy, attenuation length of the emitted photons, energy resolution, and compatibility with a specified photomultiplier. Ideally, all photons produced in the scintillator in its interactions with neutrons would be detected by the photomultiplier with $100 \%$ efficiency. In this case, the best scintillator would simply be the one characterized by the highest light output. However, much of the light emitted by the luminescent scintillator can subsequently be absorbed by the walls of the detector tank or be lost through self-absorption by the scintillator. The transparency of a given scintillator to its own emission spectrum determines the attenuation length. For small detectors, this is rarely ever a problem, since the distance between the origin of the scintillation light and the photocathode is small. However, for large detectors such as the SuperBall, a small value for the attenuation length can significantly reduce the number of photons reaching the photomultiplier. This effect cannot simply be counter- acted by increasing the light output of a given scintillator, because this also decreases the attenuation length. Hence, a compromise must be reached which maximizes simultaneously the total number of photons produced and the attenuation length. Finally, the probability that a photon will be measured by the photomultiplier, once it reaches the photocathode, is determined by the energy of the photon, due to the fact that the spectral sensitivity of the photocathode is strongly dependent on wavelength. 


\section{EVALUATION OF SCINTILLATOR PROPERTIES}

\section{A. Characteristic Absorption and Emission Spectra}

\section{Experimental Setup and Measurements}

A Perkin-Elmer fluorescence spectrophotometer was used to measure the emission spectra of gadolinium-loaded organic liquid scintillator made by either Bicron Corporation (BC-521) or National Diagnostics (ND-309). A schematic diagram of the spectrophotometer used is shown in Fig. 1. A xenon lamp is the source of excitation of the scintillator sample, having the capability of providing light over a wavelength range of $200-1200 \mathrm{~nm}$. The excitation wavelength is preset to a fixed value, in this case to $270 \mathrm{~nm}$, corresponding to the maximum absorbance wavelength of the sample. The excitation monochromator viewing the xenon lamp selects out a narrow band of wavelength(s) and returns a beam of essentially monochromatic light to the sample. The molecules in the sample absorb the radiation and emit photons, as they decay back to their ground state. The emitted photons are collected at the emission monochromator, where they are sorted according to wavelength and sent to be viewed by the photomultiplier [4]

The absorption spectrum of the scintillator was measured with a Perkin-Elmer Lambda Six absorption spectrophotometer, whose operation is similar to the fluorescence spectrophotometer described previously. In this instrument, a beam of polychromatic light, also produced with a xenon lamp, is passed through the sample. By measuring, with a photomultiplier, the intensity of light transmitted by the sample and comparing it to the intensity of light incident on the sample, the amount of light the sample absorbs can be computed. Before being viewed by the photomultiplier, the polychromatic light is collected and filtered with a monochromator. In order to accurately evaluate the emission and absorption spectra of the scintillator samples, each spectrophotometer must first be calibrated. A 10-3M anthracene sample dissolved in cyclohexane was chosen as the calibration standard for the fluorescence spectrophotometer because of its well known and defined emission spectrum. The characteristic emission peaks at 400 and $422 \mathrm{~nm}$ can be observed from the results of this calibration displayed in Fig. 2 [2]. The absorption spectrophotometer was calibrated with a sample of holonium glass, not shown here, and found to be in agreement within $0.3 \mathrm{~nm}$ of the expected results.

\section{Results}

A comparison of BC-521 and a first batch of ND-309 scintillator, given in Fig. 3, reveals a lower light output from the original sample received from National Diagnostics. In addition, its maximum emission wavelength is red-shifted by $12 \mathrm{~nm}$ from the BC-521, making it less compatible with the Thorn EMI (model 9390KB07) photomultipliers to be used with the 
SuperBall. Since then, a second test batch of fluid was obtained from National Diagnostics, with a similar compound as used in BC-521, yielding both, a somewhat higher light output and a more desirable wavelength for the emission peak (Fig. 4). Results for a third and final test batch of ND-309 was obtained which used the same formula as batch number two but with the addition of wavelength shifters, are also shown in Fig. 4. Although the modification from batch two to three of ND-309 caused a slight decrease in light output, the maximum light output of the latter sample is still comparable to that of BC-521, and its emission peak is $4 \mathrm{~nm}$ blue-shifted past BC-521. Comparing the emission spectra of the various fluids to the spectral sensitivity of the Thorn EMI photomultiplier shown in Fig.5, the third batch of ND-309 clearly gives the best spectral match.

Organic liquid scintillators are extremely sensitive to impurities, especially to oxygen from the air and to iron from the walls of the container. Such impurities can lead to chemical quenching which reduces the efficiency of the neutron detector by interfering with the energy transfer process [2]. Therefore, special care must be taken in the storage and transfer of the fluid. The techniques described above can be employed to monitor the amount of contamination and, therefore, the decline of the overall efficiency of the scintillator over time. A sample of BC-521 used repeatedly over a period of ten years in the Rochester Neutron Multiplicity Meter (NMM) was measured in the same setup and compared to a fresh sample of BC-521. The comparison of these two spectra shown in Fig. 6 proves that the scintillator has become only weakly contaminated. The retention of the high quality of the scintillator suggests that the storage and handling procedures employed in the operation of the Rochester NMM are appropriate.

The absorption spectra of both the second and third batch of ND- 309 and of the used and fresh samples of BC-521 are displayed in Fig.7. The purpose of measuring the absorption spectra is to determine the attenuation length of each sample. Light emitted from a scintillator may be reabsorbed by the scintillator if its emission and absorption spectra significantly overlap. This phenomenon, referred to as self-absorption, determines the attenuation length of a material. While the absorption spectrum of the second batch of ND- 309 extends to higher wavelengths than the others measured, its emission peak is also red-shifted by approximately the same amount. Therefore, no significant difference in the average attenuation length is observed for the different fluids measured.

\section{B. Energy Resolution Measurements with a ${ }^{22} \mathrm{Na}$ Gamma Source}

The technical objective to be accomplished with a neutron calorimetric detector is to individually distinguish and therefore, count the neutrons emitted in a reaction and to convert the total neutron kinetic energy into detectable light. This latter information is most useful if the detectable light is an approximately linear or at least a monotonic function of the energy deposited by the neutrons in the scintillator. In addition, the energy resolution is an important property of a detector which depends on the scintillator, will, hence vary 
from one scintillator to another. One obvious and simple way to acquire the corresponding information is to calibrate the response of the scintillation detector system with a radioactive source of gamma rays of known energy. As photons penetrate through the liquid organic scintillator, they interact primarily through Compton scattering. The photopeak efficiency is comparatively small, due to the minimal effective $Z$ of the scintillator. As a result, the light output spectrum of the scintillator contains only Compton edges and no discernible photopeaks [5].

\section{Experimental Setup and Measurements}

Cylindrical scintillator cells of dimensions 1.5 " $\mathrm{x} 5$ " diameter were filled with the various samples of ND-309 and BC-521 and coupled to a fast Philips (model XP2041) photomultiplier. An identical scintillator cell, factory-filled with NE-213, was used as a standard for comparison. A larger scintillator cell of dimensions 6.5 " $\times 6$ " diameter was filled with the same samples and coupled to either a fast Philips (model XP2041) or Thorn EMI (model 9390KB07) photomultiplier and subjected to the same test measurements. In some additional tests the inside walls of the scintillator cell was covered with a 1/32" sheet of teflon. Each scintillator was irradiated with a ${ }^{22} \mathrm{Na}$ gamma ray source. The signal was sent to a preamplifier, which converts the charge pulse from the photomultiplier to a millivolt signal which is then amplified, digitized and recorded in an multichannel analyzer.

\section{Results}

Well-defined Compton edges, corresponding to the 0.511 and $1.27 \mathrm{MeV}$ photons characteristic of a ${ }^{22} \mathrm{Na}$ source were observed for NE-213, BC-521, and the second batch of ND-309 with the smaller scintillation cell (see Fig.8). However, the absence of Compton edges in the measurement involving the first batch of ND-309 suggest a severe contamination of this scintillator fluid with material of the cell walls, due to a chemical aggressivity of the fluid. After a relatively short contact time (only a few hours), this contamination led to a visible discoloration of the scintillator liquid. Thus this scintillator was disqualified for the intended purpose (Fig. 9). NE-213 scintillation cells have been used previously in neutron time- offlight measurements. As shown in Fig.8, the high light output of this scintillator provides an excellent energy resolution for both neutrons and gamma rays. Both, the BC-521 and the second batch of ND-309 give reasonably good energy resolution in comparison.

The energy resolution obtained with the larger, 6.5" 6 " diameter; scintillation cell gives a more realistic idea of the performance to be expected for the SuperBall. Generally the resolution achieved with a given scintillator detector is expected to decrease as the size of the detector is increased, a result of the increased surface area of the walls of the detector where light can be absorbed. One way to increase the quantum efficiency of such a detector and, hence, its energy resolution consists in coating the inner surfaces of the scintillator cell 
with some type of highly reflective material. Fig. 10 illustrates the dramatic improvement of the resolution of the detector with the large cell experienced by lining the inner walls with white sheet teflon. The loss of a significant portion of total light output, in the case of the uncoated scintillator cell, is evident by the absence of any discernible Compton edge in the light output spectrum. On the other hand, when lined with the reflective material, the cell gives rise to well-pronounced Compton peaks.

The results of energy calibrations carried out for BC-521 as well as both samples of ND309 , in the teflon-coated large scintillator cell, are shown in Fig. 11-12. The scintillator cell was coupled to either the fast Philips (Fig. 11) or the Thorn EMI (Fig. 12) photomultiplier. In both cases, high resolution was achieved for all BC-521 and ND-309 samples.

\section{EVALUATION OF DIFFUSE REFLECTANCE OF COATING MATERIAL}

The investigation of reflective material to coat the SuperBall was prompted by the the results from the energy calibration measurements discussed previously. Without lining any of the interior walls of a scintillator cell with reflective material, the 6.5 " $\times 6$ " diameter neutron detector had such poor resolution that neither the .511 nor $1.27 \mathrm{MeV}$ Compton edge was resolved. The poor resolution can be attributed to the absorption of light from the inner surface of the scintillator cell. Light is emitted isotropically from the luminescent centers of the liquid scintillator. Only a portion of this light will be directed toward the photomultiplier, while some of the photons will be emitted toward the walls of the container where they can be absorbed. If the inner walls of the detector are covered with a reflective material, these photons can be redirected through reflection. Teflon and alumina spray were selected as possible lining or coating materials based on their high reflectivity, compatibility with the organic scintillator, cost effectiveness, and ease of application.

The diffuse reflectance of each material was measured by Hitachi Instruments Inc. with a spectroreflectometer. This instrument is essentially a spectrophotometer with an integrating sphere. A monochromatic beam of light is passed into the integrating sphere and directed at the sample. The interior of the sphere is coated with magnesium oxide so that all of the reflected light is collected at the photomultiplier. Light traps are positioned at a 90 degree angle with respect to the sample to eliminate any contributions from specular reflectance [6]. The information from this test gives the percentage of light a particular material can reflect back into the scintillation tank as a function of wavelength. In order to evaluate the contribution of the reflective coating, the diffuse reflectance of the uncoated surface of the neutron detector, either aluminum or stainless steel (304SS), must be measured as well.

\section{A. Results}

As indicated by the results of the diffuse reflectance measurements in Fig. 13, a lining or coating of the scintillator tank leads to at least a factor of 2-3 increase in light reflectance 
and, therefore, a significant decrease in light losses, for the relevant wavelength range of 330-450 nm. Among the two possible coating materials, teflon shows a somewhat higher degree of reflectivity in this wavelength region.

\section{CONCLUSION}

The energy calibration and spectroscopic measurements discussed above suggest that both the BC-521 and the second or third batches of ND-309 scintillator have properties that make them suitable for applications with the SuperBall neutron calorimeter. A calorimeter filled with such liquid scintillator would provide efficient means of determining the total kinetic energy of neutrons dissipated in the liquid, as well as their multiplicity. All three fairly transparent scintillators show only small overlaps between their emission and absorption spectra, and provide for comparable energy resolutions if used in conjunction with either the fast Philips (model XP2041) or the Thorn EMI (model 9390KB07) photomultipliers. The position of the emission peak of ND-309 (batch 3) occurs at approximately $90 \%$ of the maximum sensitivity of the Thorn EMI (model 9390KB07) photomultiplier, making this a well-matched combination that will (likely) be adopted for the SuperBall detector.

The results of the tests performed for the BC-521 scintillator, used over the past decade in heavy-ion reaction studies, showed only slight deterioration in total light output and essentially no decrease in attenuation length. The differences are small enough such that the efficiency of the detector has not been altered significantly over time, even though the detector has been drained and filled several times. This technique will be employed in the future as a method of monitoring the SuperBall scintillator. In addition, these results show that the stainless steel, which is the proposed material for the tanks of the SuperBall, leads to little if any contamination of the scintillator.

Finally, the results of the diffuse reflectance study indicate that coating the interior of the SuperBall will greatly enhance the efficiency of such a detector. While either the teflon lining or alumina spray of the tank walls would provide adequate reflectance of the detector surface, lining with sheet teflon has been chosen because of the slightly higher reflectance, the lower cost and the ease of application achieved.

Likewise, 900 liters of the third batch of ND-309 have been ordered to be used in one final series of test in the Rochester NMM. Information for BC-521 with this neutron detector is already available as a standard for comparison. If the ND-309 scintillator in the NMM should, as expected, confirm extrapolations based on results of the preliminary test discussed above, it will likely be chosen as the SuperBall scintillator, based on its overall efficiency as well as its cost effectiveness.

This work was supported by the U.S. Department of Energy under Grant No.DE-FG0288ER40414. 


\section{REFERENCES}

[1] B.C. Diven, H.C. Martin, R.F. Taschek, Terrell, Phys. Rev. 101, 1012 (1956)

[2] J.B. Birks, The Theory and Practice of Scintillation Counting (Pergamon Press, N.Y. 1964).

[3] G.F. Knoll, Radiation Detection and Measurement (John Wiley and Sons, N.Y. 1979)

[4] Instructions manual for the Perkin-Elmer Models MPF-43/44 and MPF 43A/44A Fluorescence Spectrophotometer (Perkins-Elmer Corp., Norwalk 1975).

[5] W.R. Lec, Techniques for Nuclear and Particle Physics Experiments (Springer-Verlag, N.Y. 1987).

[6] H.H. Willard, L.I. Merritt Jr., and J.A. Dean, Instrumental Methods of Analysis (D.Van Nostrand Co., N.Y. 1974). 


\section{FIGURES}

FIG. 1. Schematic diagram of Perkin-Elmer spectrophotometer.

FIG. 2. Emission spectrum of $10-3 \mathrm{M}$ anthracene solution dissolved in cyclohexan.

FIG. 3. Emission spectra of BC-521 and the first batch of ND-309 scintillator.

FIG. 4. Emission spectra of BC-521 and both the scond and third batch of ND-309 scintillator.

FIG. 5. Spectral Response of Thorn EMI (model 9390KB07) photomultiplier.

FIG. 6. Emission spectra of BC-521 scintillator used previously in experiments and of a fresh sample of the same BC-521 scintillator.

FIG. 7. Absorption spectra of both samples of BC-521, and the second and third batch of ND-309 scintillator.

FIG. 8. Energy spectra of a Na-22 source obtained with NE-213, BC-521, and the second batch of ND-309 scintillator in the 1.5 " $\times 5$ " diameter cell.

FIG. 9. Energy spectrum of a NA-22 source obtained with the first batch of ND-309 scintillator in the 1.5 " $\times 5 "$ diameter cell.

FIG. 10. Energy spectra of Na-22 source obtained with BC-521 scintillator in the 6.5 " $\times 6$ " diameter cell with and without teflon lining. In both spectra the fast Philips (model XP2041) photomultiplier was used.

FIG. 11. Energy spectra of a ${ }^{22} \mathrm{Na}$ source obtained with BC-521 and the second and third batch of ND-309 scintillator in the 6.5" $\times 6$ " diameter, teflon lined cell. In these spectra the fast Philips (model XP2041) photomultiplier was used. 
FIG. 12. Energy spectra of a ${ }^{22} \mathrm{Na}$ source obtained with BC-521 and the second and third batch of ND-309 scintillator in the 6.5" $\times 6$ " diameter, teflon lined cell. In these spectra the Thorn EMI (model 9390KB07) photomultiplier was used.

FIG. 13. Diffuse reflectance spectra of teflon lining, alumina coating, aluminum and stainless steel. 

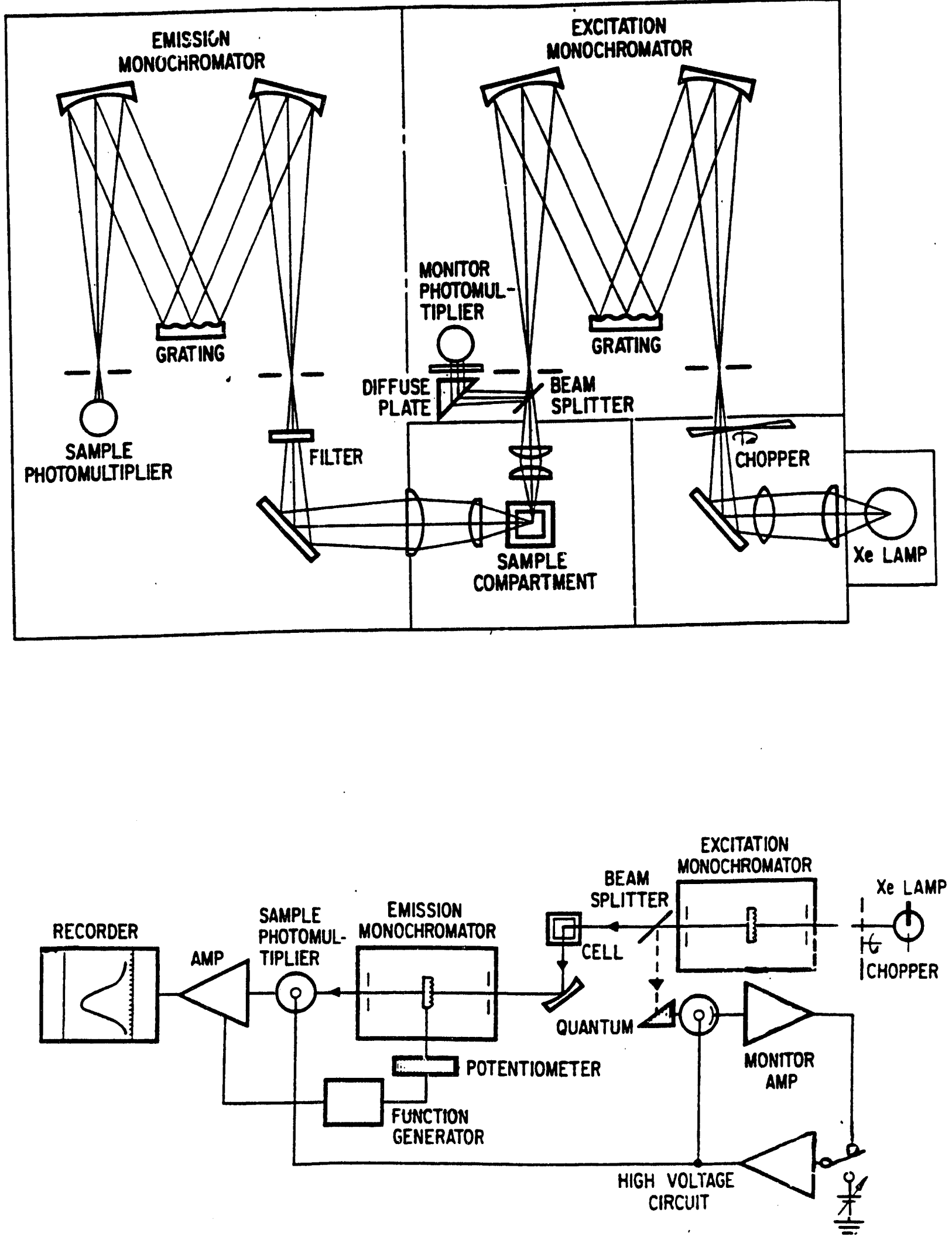

Figure 1 


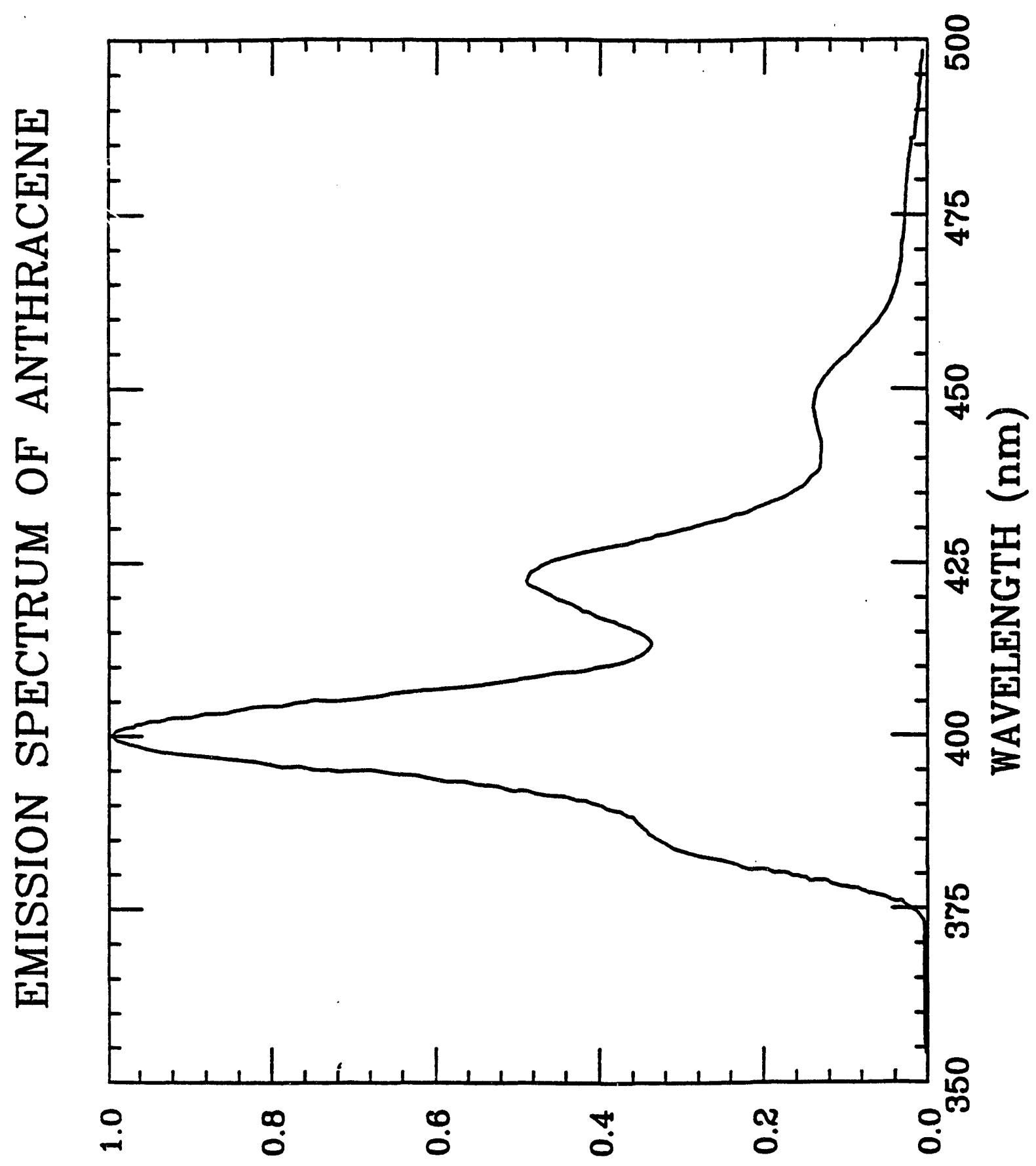

XIISN'HLNI BAILVT'HA

Figure 2 
EMISSION SPECTRA OF BC-521 AND ND-309

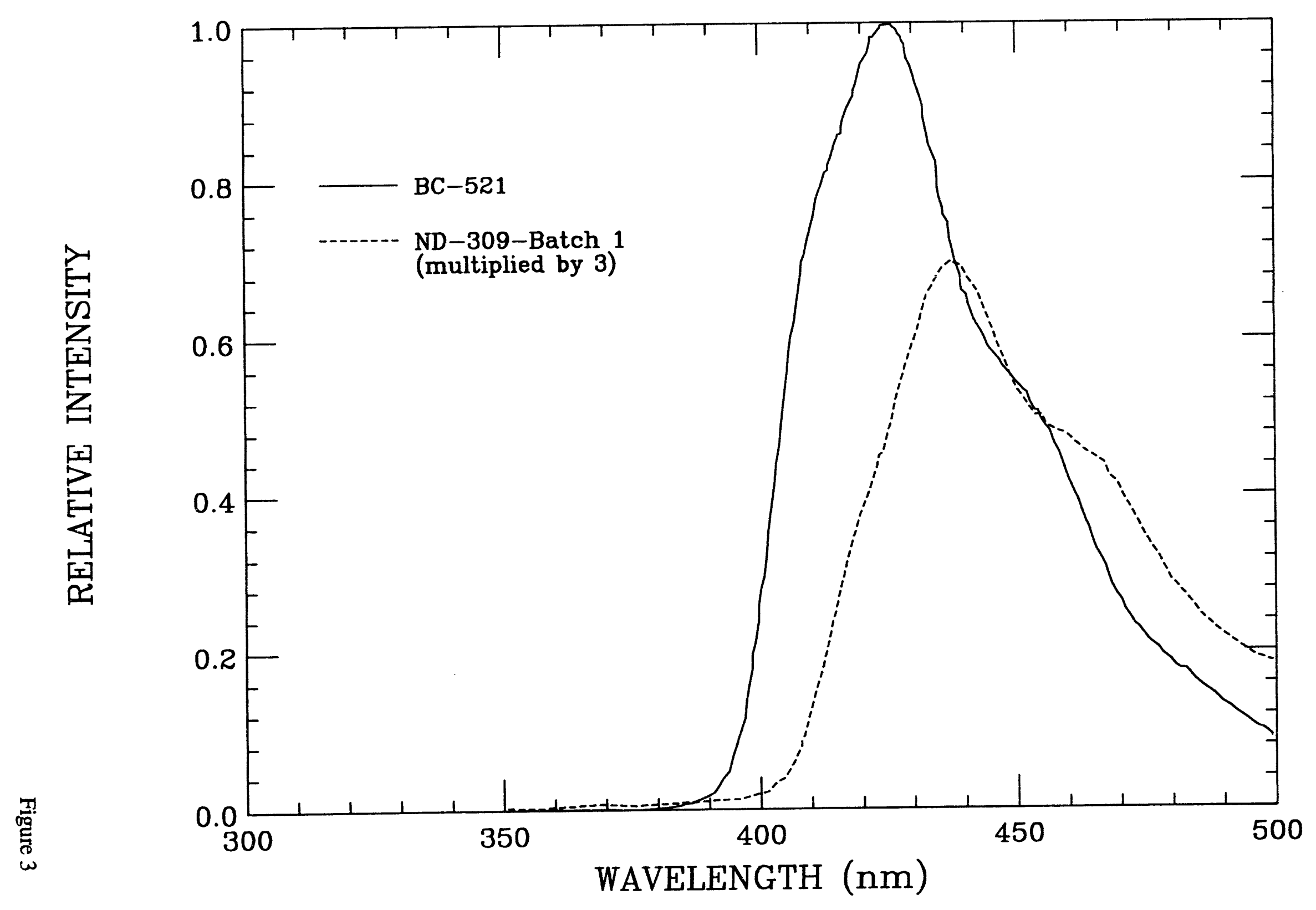


EMISSION SPECTRA OF BC-521 AND NEW ND-309

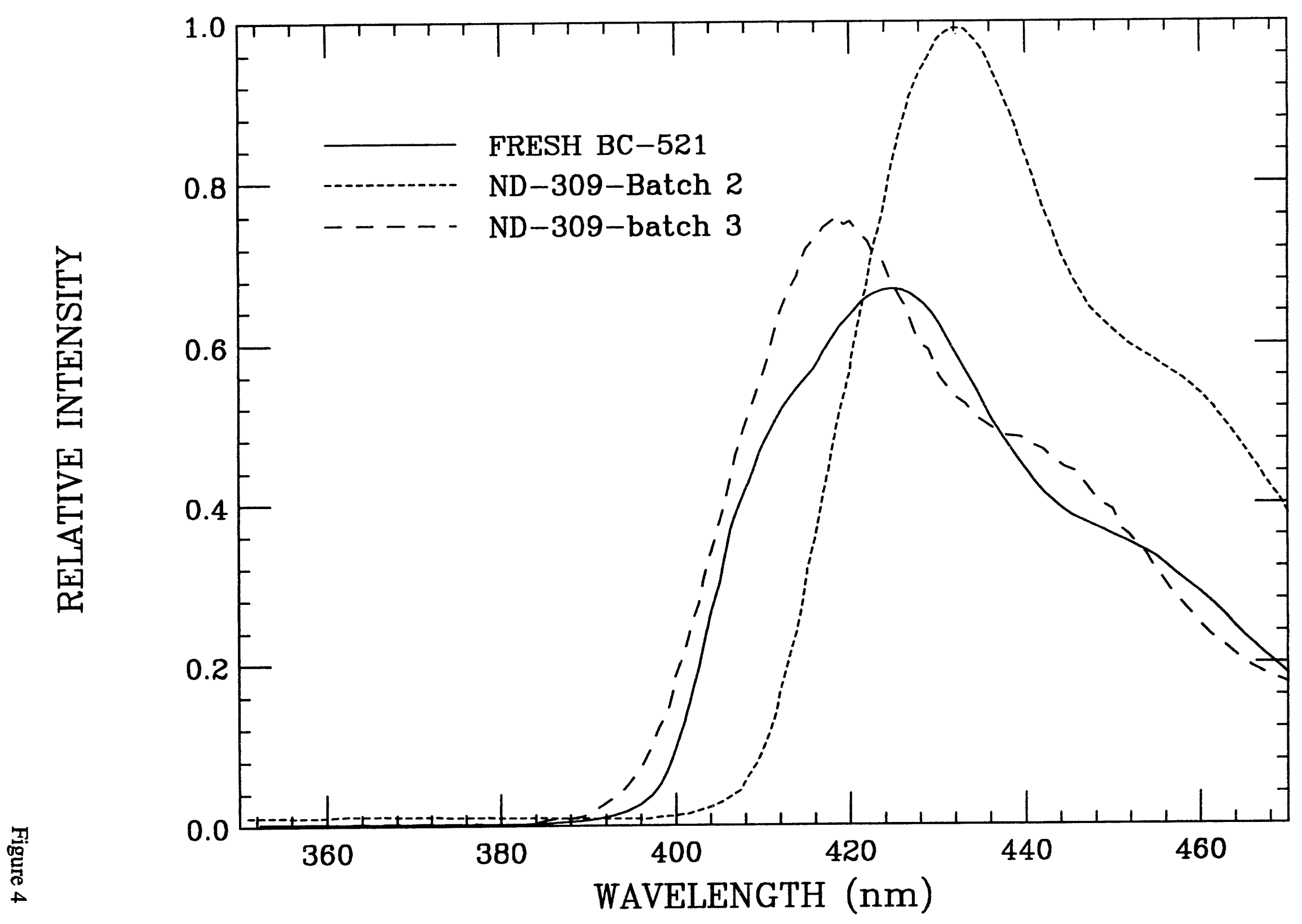




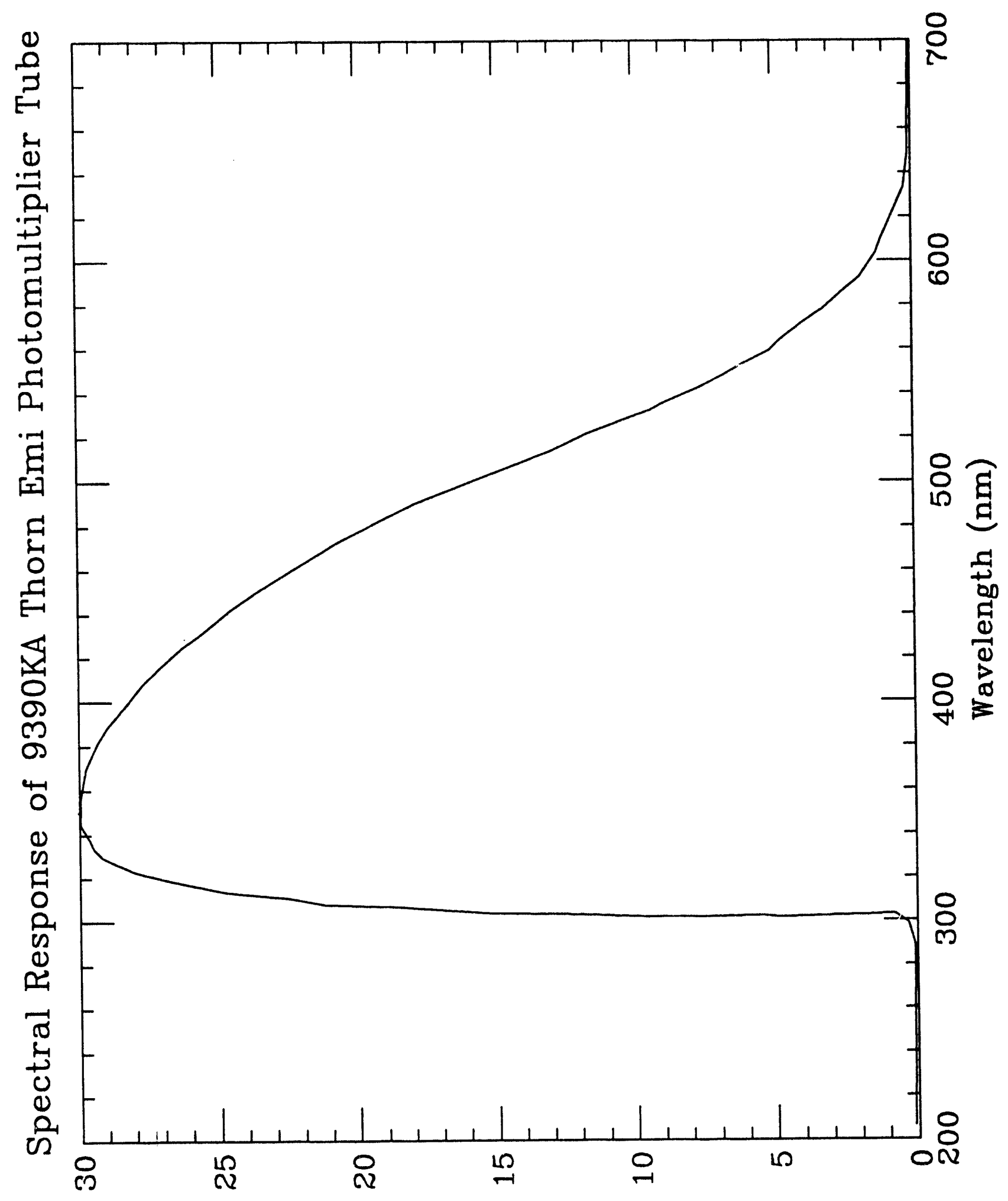

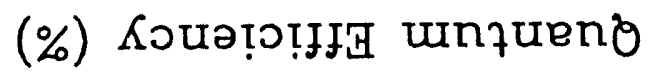

Figure 5 


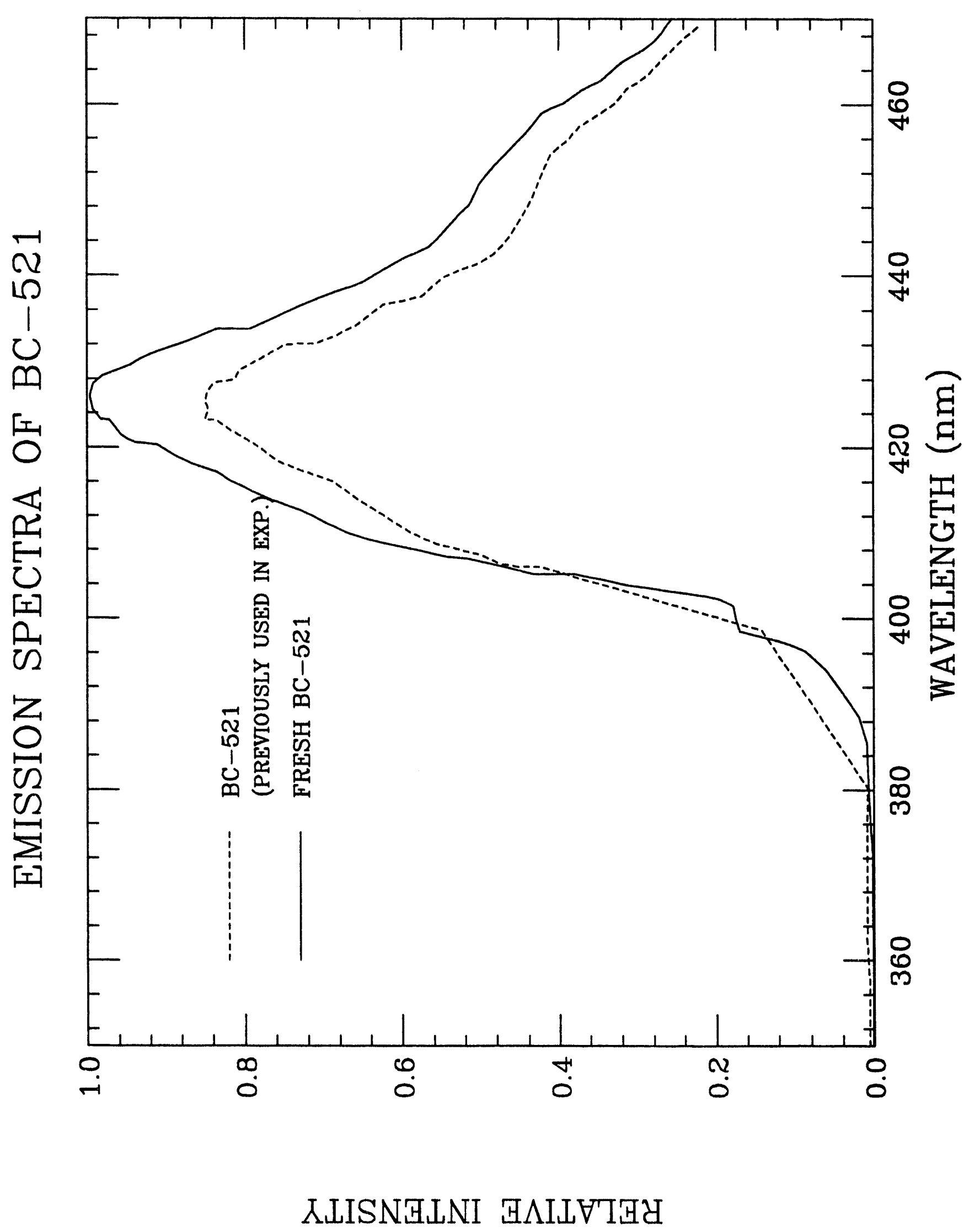

Figure 6 


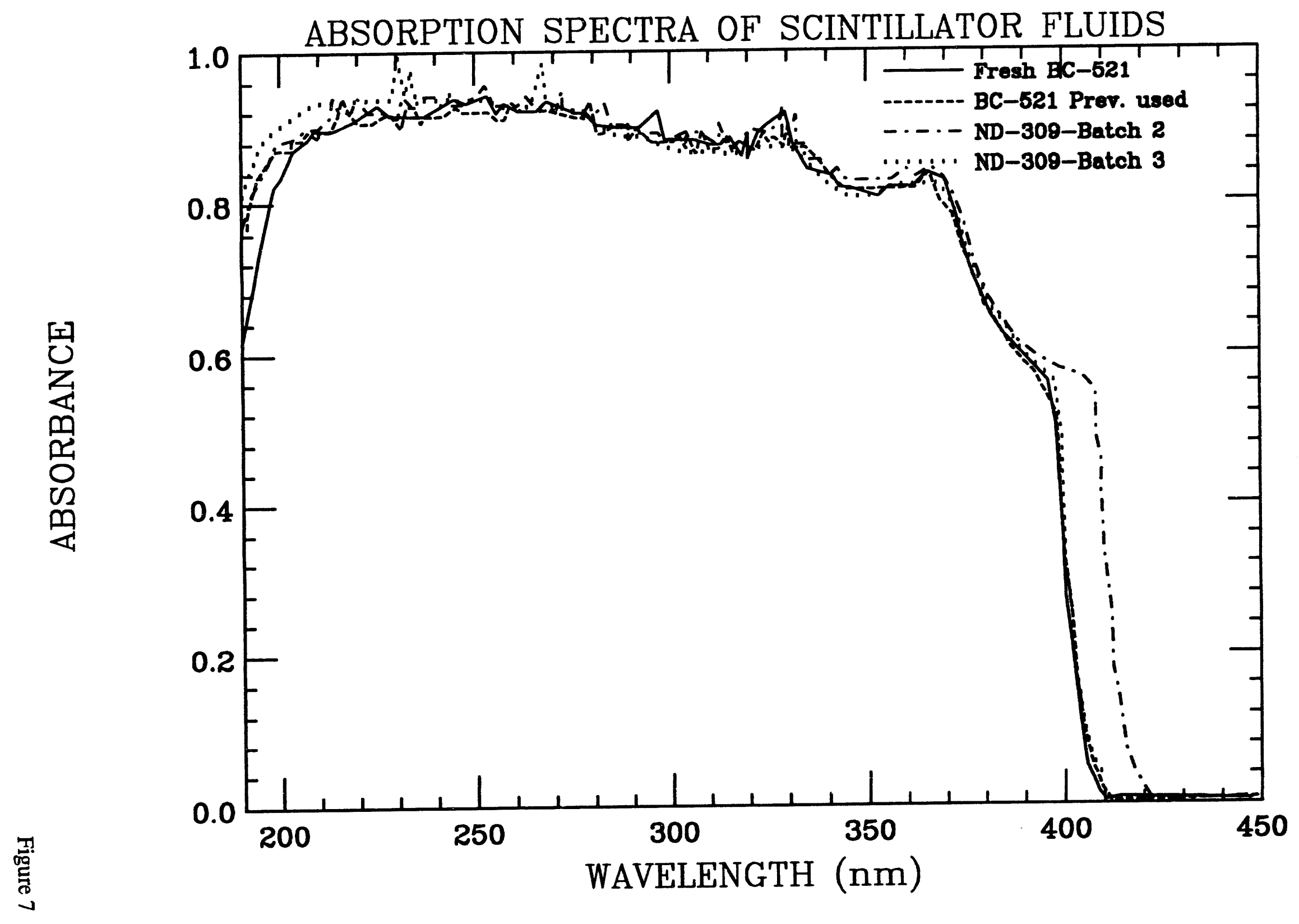




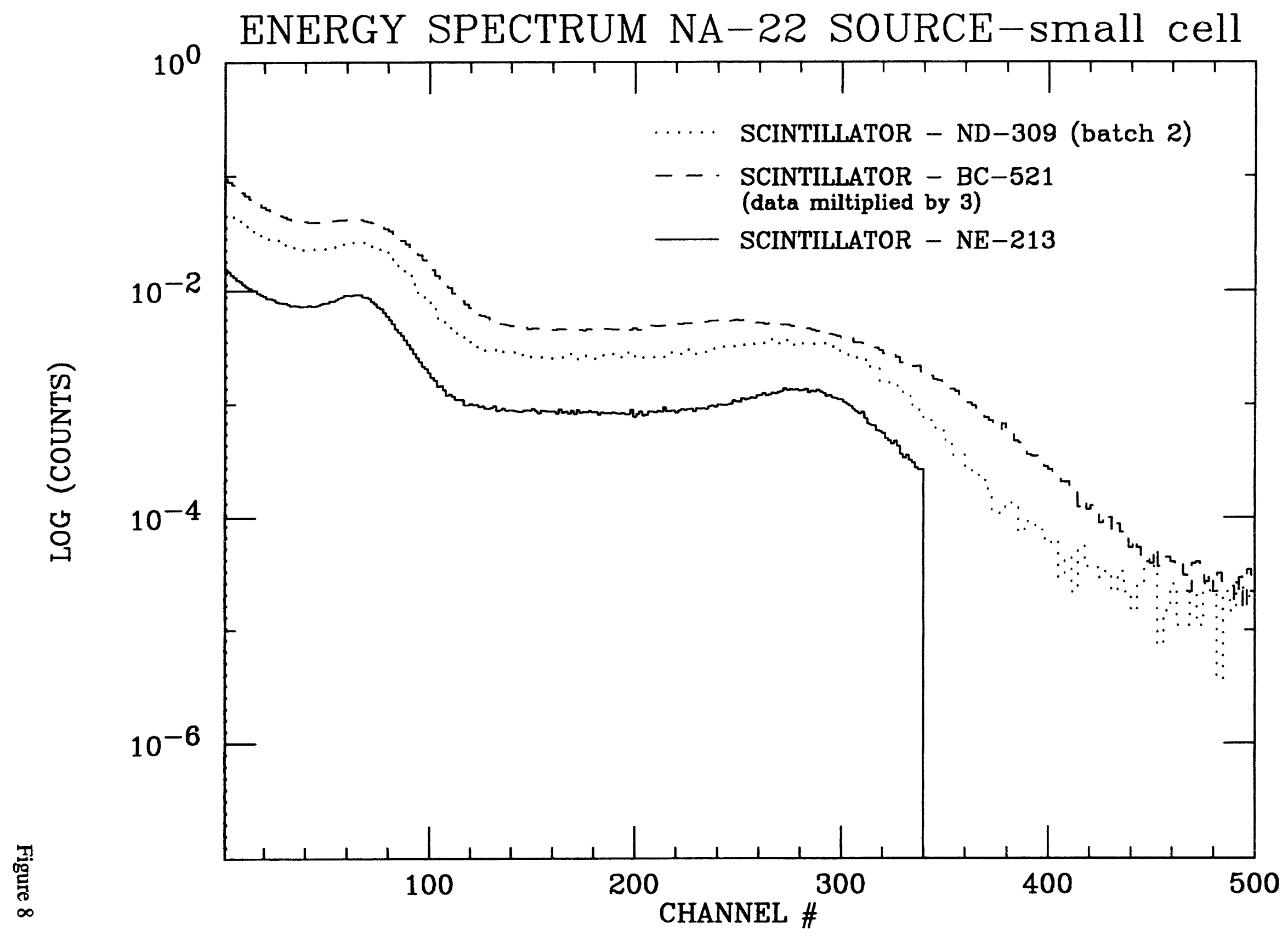




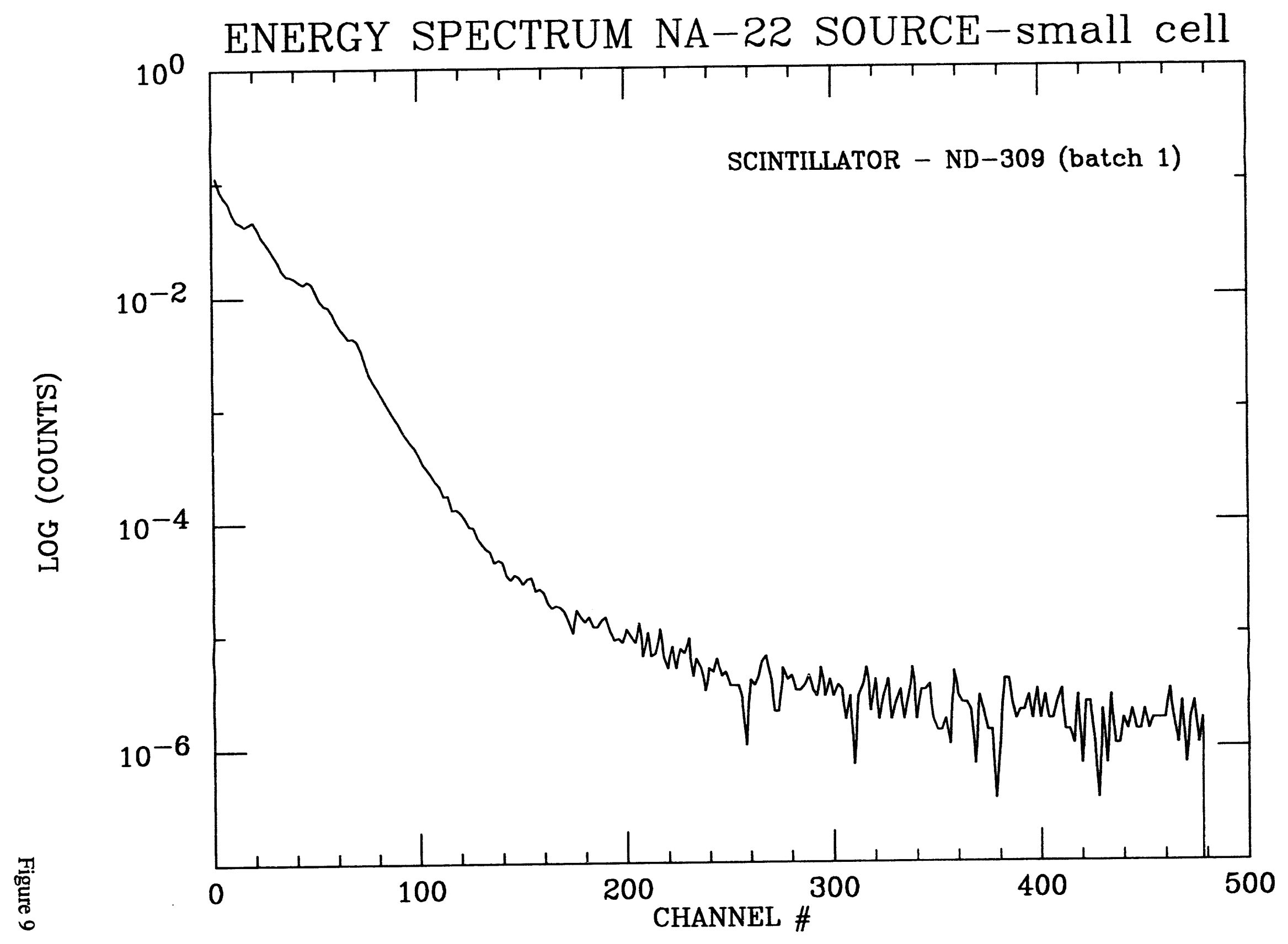




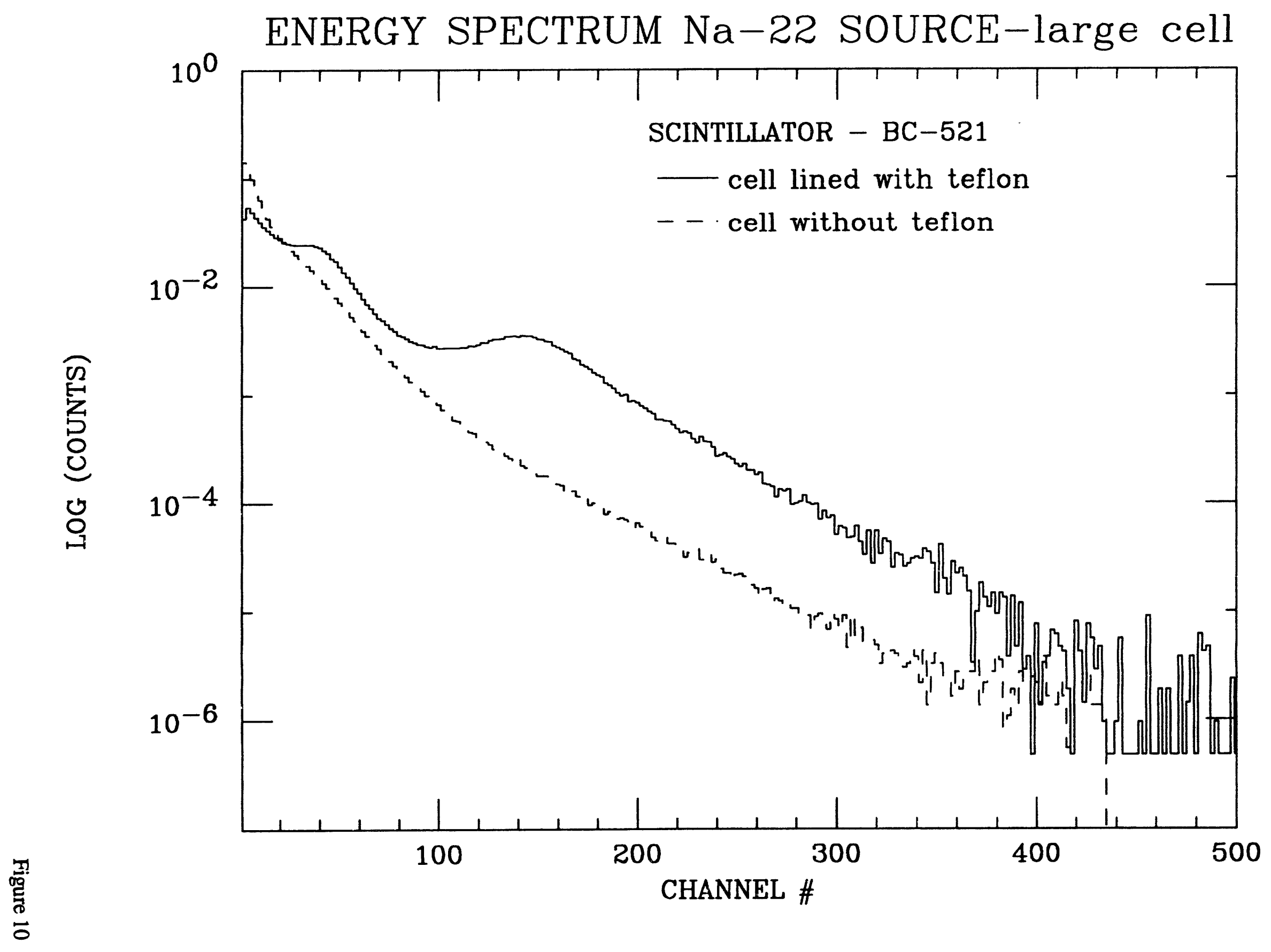




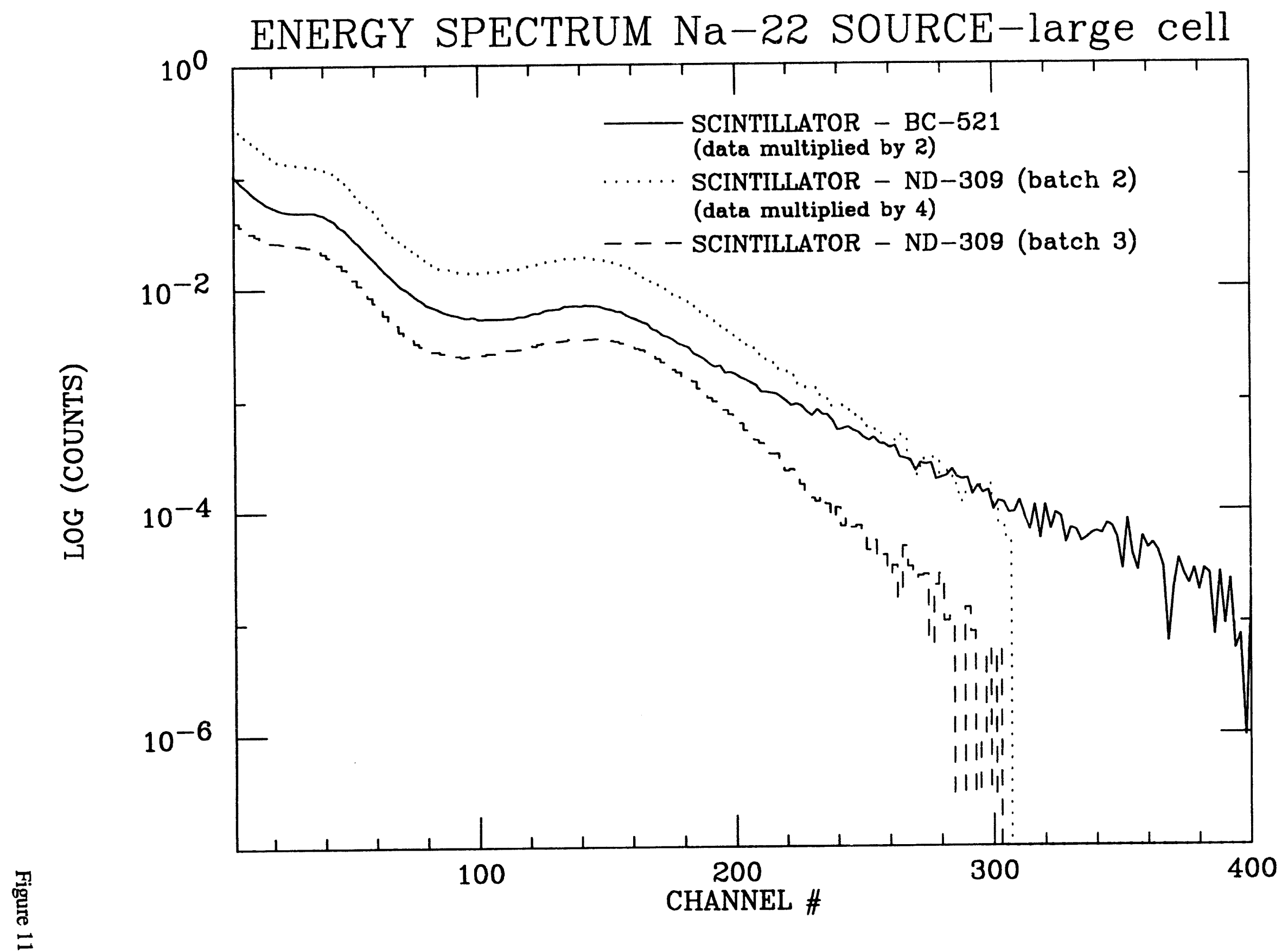




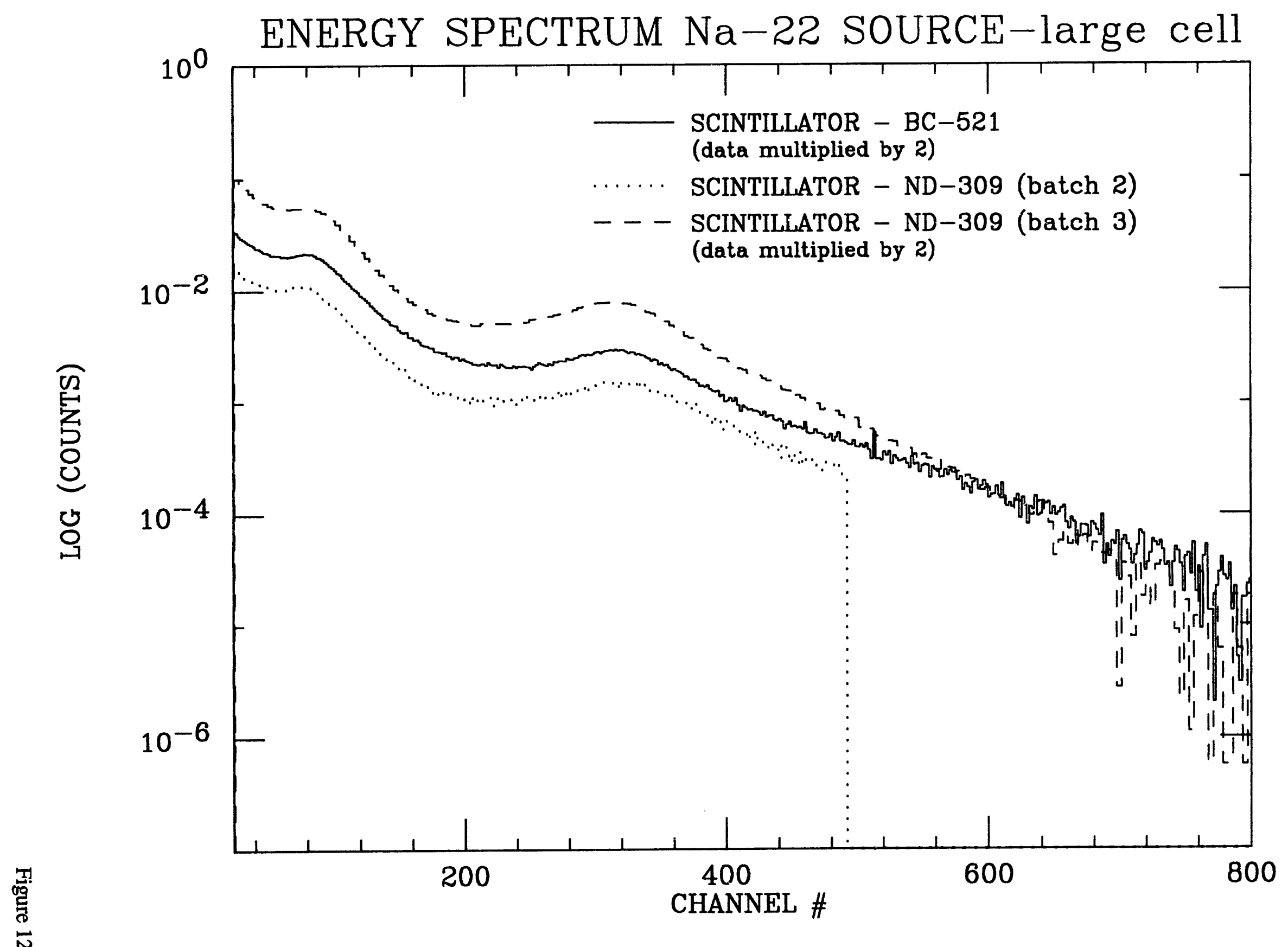




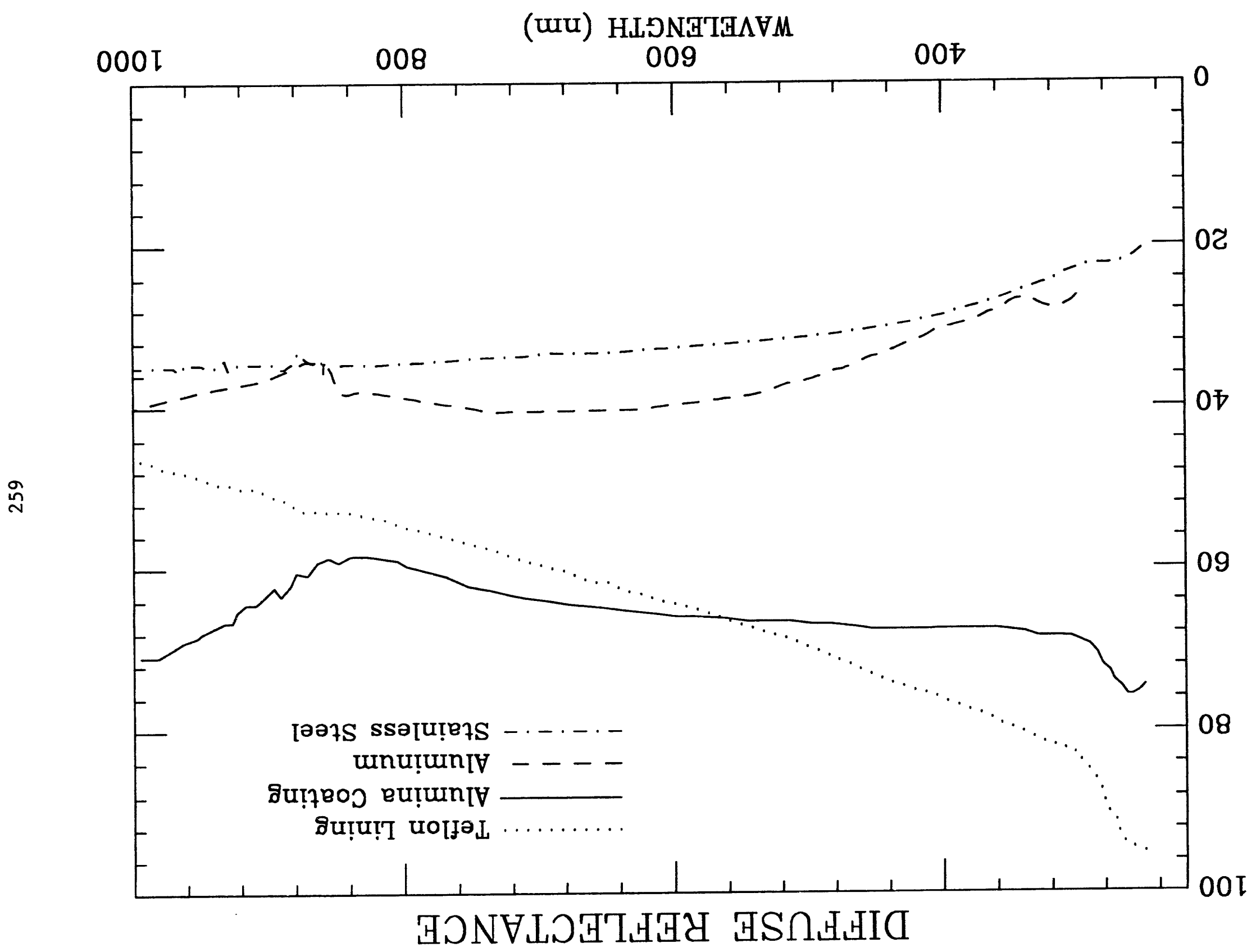

ㅇํㅂ 


\title{
Measurement of the Interaction of Cosmic-Ray $\mu^{-}$ with a Muon Telescope
}

\author{
Karen Ho \\ Senior Study Project \\ Prof. W. Udo Schroeder, Supervisor \\ Dept. of Chemistry and Nuclear Structure Research Laboratory \\ University of Rochester, Rochester, N.Y. 14627 \\ Dr. Mandeville, Advisor \\ Allendale Columbia, Rochester, N.Y. 14618 \\ (June 9, 1993)
}

\begin{abstract}
A muon telescope is being used to identify $\mu^{-}$by measuring their interaction with the telescope's lead target. The techniques being used in this experiment are similar to those which will be used to identify $\mu^{+}$, which decay in the process $\mu^{+} \rightarrow e^{+}+\nu_{\mu}+\nu_{e}$. The positrons from this decay will be used in experiments with Superball, a large scintillation detector currently under construction.
\end{abstract}

\section{INTRODUCTION}

This is a report on a senior study project conducted at the Nuclear Structure Research Laboratory at the University of Rochester.

Negative $\left(\mu^{-}\right)$and positive $\left(\mu^{+}\right)$muons are major components of cosmic radiation that continuously bombards the Earth. The presence of these charged particles affects studies of nuclear reactions, such as those to be done with Superball, a highly sensitive neutron detector currently under construction by NSRL's Nuclear Chemistry Group. 
The object of this research is to identify negative muons in cosmic-ray showers by measuring their interactions with the muon telescope target. The process being used to do this is similar to the one that will identify positive muons. The positrons $\left(e^{+}\right)$from the decay of these muons will be used to trigger experiments with the Superball; the ultimate goal is to calibrate the light output of the Superball, since the positron energy spectrum is already known.

\section{EXPLANATION OF CRITICAL TERMS}

Before this report proceeds, several critical terms will be explained.

\section{A. Cosmic radiation}

Cosmic radiation refers to the highly penetrating high energy particles that continuously bombard the earth from outer space. These particles include photons and subatomic particles, such as protons and atomic nuclei. The atmosphere absorbs most radiation, but some particles have enough energy to reach the Earth's surface. (Some even continue to travel underground.) More specifically, approximately 20 particles per square centimeter reach the top of the atmosphere per second; at sea level, there is only about one particle per centimeter per second. This radiation can pass relatively harmlessly through our bodies at all times $[1]$.

\section{B. Muons}

Muons are negatively and positively charged particles that constitute a large part of the cosmic radiation detectable at the Earth's surface. They are heavy $\left(m_{\mu}=206 \cdot m_{e}\right)$ electron-like particles with a lifetime of about $2 \mu \mathrm{s}$.

In this experiment, $\mu^{-}$stopped in a lead target release energy by interacting with target atoms. Since these muons behave in a way similar to electrons, they replace electrons in the outermost orbits of the lead atoms. Muonic X-rays are released when the muons drop down into lower energy levels (or orbitals); when the muon reaches a low enough orbital, it is captured by the lead nucleus. The $\mu^{-}$interacts with the protons inside it to form neutrons and neutrinos $\left(\nu_{\mu}\right.$ and $\left.\nu_{e}\right)$, which are lightweight, neutral particles. Neutrons and $\gamma$-rays are emitted from this interaction. This capture of a $\mu^{-}$by a lead atom occurs on a (70-90)ns time scale. Figure 1 illustrates $\mu^{-}$capture.

$\mu^{+}$behave in a slightly different manner. They cannot be captured by a nucleus (it is positively charged) but decay in the process $\mu^{+} \rightarrow e^{+}+\nu_{\mu}+\nu_{e}$. ( $\mu^{-}$also decay, but in the process $\mu^{-} \rightarrow e^{-}+\nu_{\mu}+\bar{\nu}_{e}$ ) The positrons from $\mu^{+}$decay will be used to trigger the scintillators in the Superball. 


\section{Scintillation Detectors}

Any scintillating material emits a small flash of light, or a scintillation, when struck by a charged particle or photon. This occurs because the particle excites the atoms in the scintillator, causing light to be emitted when the energy is relost. A scintillation detector consists of scintillating material coupled to a photomultiplier tube. In this experiment, three detectors are used as muon telescope counters and one as a neutron detector. The neutron detector and one of the telescope counters use a liquid scintillator; the other two detectors use plastic. The light from the plastic detectors are transmitted to the photomultipliers by light guides, pieces of plastic specially shaped to accommodate the thin edges of the scintillators and round surfaces of the photomultipliers. The liquid scintillators do not need any light guides. Figure 2 is a drawing of a plastic scintillator used in the experiment. A photomultiplier tube, shown in Figure 3, converts the photons from the scintillator into electrons, which form a current that can be amplified and analyzed [2].

\section{EXPERIMENTAL METHOD}

This experiment began with the construction of a muon telescope to identify negative muons and measure their capture products. This telescope consists of three scintillation counters, a moderator, target, and neutron detector. The purpose of the counters is to detect the passage of charged particles by the light flashes produced in the scintillators.

The following is a description of the set-up of the telescope (shown in Figure 4). Two detectors, labelled counter 1 and counter 2, are placed on top of each other. Six inches of lead are stacked between them to form the moderator. The purpose of the moderator is to attenuate penetrating particles. A target, which consists of two inches of lead, is placed beneath counter 2, while counter 3 is placed under the target. The neutron detector is under, and in line with, all the other detectors.

These detectors are connected to various electronic modules, or devices, that analyze and count the signals. If a light flash is detected simultaneously in counters 1 and 2 (the signals are in coincidence), but not in counter 3 , then it is assumed that a charged particle completely penetrated the first two counters and the moderator but not the target. A logic module is used to generate a proper electronic signal $(1 \cap 2 \cap \overline{3})$ indicating this 'stop' of a particle in the target. In actuality, this signal only indicates that a particle did not travel straight through the target and counter 3 ; there are no counters on the other four sides of the target to determine if the particle scattered out. However, for this experiment, it can be assumed that at least some particles were not scattered out and remained in the target. Numerous trials of varying moderator and target thicknesses were conducted to determine which combination would maximize the rate of muon stops in the target and minimize the amount of less penetrating cosmic ray components. A six inch thick moderator and two inch thick target were chosen because this combination produced a clear bump in a plot which 
compared the fractions of incoming particles stopped by various moderator thicknesses with different target thicknesses, which is shown in Figure 5. While some target thicknesses stopped a larger fraction of particles than a two inch one, varying the moderator thicknesses did not seem to affect the fractions stopped to a large degree-only in the two inch thick target was this apparent. With this target, the six inch moderator stopped the highest percentage of particles (about 69\%). Of course, some particles are energetic enough to penetrate all three counters. However, they do not produce the proper signal $(1 \cap 2 \cap \overline{3})$ and are not included in any measurements.

The desired electronic signal is used as a start signal for the Time-to-Amplitude Converter, or TAC, which is an electronic clock. If the charged particle stopped in the target is a negative muon, it interacts with target atoms and atomic capture products (muonic $\mathrm{X}$-rays, $\gamma$-rays, and neutrons) will result. These can penetrate counter 3 and be detected in the neutron detector underneath it. A signal from this detector is used to stop the TAC. The TAC generates a signal with an amplitude proportional to the time elapsed between the start signal (the time of the muon stop) and stop signal (the time of nucleus capture). Increasing the time between the start .... stop signals (by delaying the stop signal, for example) will increase the amplitude (voltage) of the output. A multichannel analyzer (MCA) (or computer workstation) is used to accumulate events and displays the number of counts associated with a particular delay time. In general, the MCA displays a histogram of the number of counts (or signals) associated with a particular voltage, but since the voltage of the signal from the TAC represents the delay time, the MCA can display the number of counts (on the $y$-axis) for a particular delay time (on the $\mathrm{x}$-axis).

Figure 6 is a diagram of the electronics being used in this experiment. All signals originate in the anodes of the detectors, which produce negative pulses. The analog pulses from counters 2 and 3 then pass through a Leading Edge Discriminator (LED) and are converted into logic time pulses. These are just 'yes' or 'no' signals for pulses, and their amplitudes do not depend on the energies of the particles. Figure 7 includes examples of analog and logic time pulses. The signals from counter 1 and 4 (they are liquid scintillators) are converted into the same time pulses by Constant Fraction Discriminators (CFDs), which are triggered by the passage of a certain fraction of each pulse. The pulses from all three counters then pass through a logic module. As explained, an output signal is generated if a particle passes through counters 1 and 2 but not 3 . This signal is used to start the TAC while a signal from the neutron detector stops it. The output generated in the TAC is then delayed and used to trigger the computer system, as shown in Figure 8. Before the computer can analyze the output, however, it must be digitized. The Analog-to-Digital Converter is a module which does this. By the time the digitized signal reaches the computer, the computer has been triggered and is prepared to receive data. Various time delays are also included in the whole electronics set-up to ensure that signals were timed properly. For instance, while a particle might travel through both counters 1 and 2, the different lengths of cable from the detectors to the logic modules (which determine coincidence) might cause a delay in a signal from one of the detectors. (Signals cover about one meter of cable every $3 \mathrm{~ns}$ ). 
Time delay modules compensate for these differences. The signals also have to be converted into different sizes and types at various times. The main reason for changing the types of signals (from analog to time, for example) is that some modules require certain types of pulses to operate. Lastly, some modules besides those just mentioned are used to generate information about incoming radiation the computer does not. For example, a logic and counting module is used to display the number of particles coincident in counters 1 and 2, while another module displays the number of particles that penetrate counters 1 and 2 but not 3. A timer is also included to time the whole process. These are helpful in calculating the percentages of particles stopped and the rates of stoppage.

\section{RESULTS OF THE EXPERIMENT}

A muon telescope was constructed that successfully detects cosmic radiation. It is important to note that cosmic-ray particles are rare; the test measurements show that counts are taken only about once every 200 seconds. Therefore, accumulating enough data often takes a day or more.

The presence of $\mu^{-}$in the telescope target is consistent with the data acquired, but needs to be qualitatively confirmed with data of higher statistical accuracy.

Figure 9 is an example of current output from the MCA. The $x$-axis displays its channel numbers. (Channels are similar to pixels on a computer screen.) Each delay time is assigned a channel number; in this experiment, it was determined that 1 channel represented an 84 ps delay time. The y-axis displays the number of counts, or yield, for each delay time. The stoppage of a negative muon could be confirmed by analyzing this kind of output. The muonic X-rays from the $\mu^{-}$interacting with the lead produce a 'prompt' peak. The decay of the muon, which occurs on a longer time scale, produces an exponentially decreasing curve to the right of this peak. In Figure 9, enhanced intensity is actually seen at longer delay times, as expected for $\mu^{-}$-capture. However, the number of counts registered in this part is still low.

In the experiment, different geometries of the telescope have been explored. For example, test measurements have been taken to see if different moderator and target thicknesses will affect the results. In addition, there has been some experimentation with a paraffin moderator instead of a lead one. Paraffin will not affect the motion of muons, but it will stop protons. The reason for this is that colliding protons transmit their kinetic energy most effectively to the light nuclei of paraffin and are thus stopped. (Lead nuclei are so massive that colliding protons scatter off them.) The reason for making these adjustments is to find a configuration that stops the most muons. If, for example, the results from the telescope with a paraffin moderator are clearly different from those taken with a lead moderator, then a reasonable conclusion would be that the particles being detected are protons, rather than muons.

Other experiments with the telescope are also being considered. Moving the target 
closer to the neutron detector, for example, might improve the results. Adding a $\gamma$-ray detector to the set-up may also have interesting results, because the $\gamma$ detector would detect characteristic $\gamma$ radiation that can be used to identify the nuclei produced in muon capture.

As this paper is being written, measurements with the muon telescope are still being made; the experiment continues as this project ends.

\section{SUMMARY}

A muon telescope has been constructed to identify $\mu^{-}$in cosmic radiation by measuring their interaction with a lead target. This telescope consists of three scintillation detector counters, a moderator (to attenuate or stop the less energetic components of cosmic radiation), a lead target, and a neutron detector (to measure capture products).

The results of this project will be helpful in experiments to be done with Superball, a very large and sensitive neutron detector. In Superball, the muon telescope target will be replaced by scintillating material. Positrons from $\mu^{+}$decay will be used to excite the scintillating material. Since the energy spectrum of positrons is already known, the light output of Superball can be calibrated.

The $\mu^{+}$to be used in Superball experiments will be identified in a process similar to the one used in this project to identify $\mu^{-}$.

\section{SOME CONCLUDING REMARKS}

It is perhaps important to note that this project did not begin immediately with the construction of a muon telescope. In fact, I began this project with scant knowledge of particle physics, and I was completely confused by even the object of this experiment.

Thus, the beginning portion of this project was devoted to introductory work. The first week was spent putting together detectors from available parts and becoming accustomed to the electronics involved (and being told repeatedly that cables are NOT wires!). I made test measurements with only one detector, and then a second, inside a light-tight box. The purpose of these measurements was to practice looking at and timing the pulses correctly. Then, they were taken out of the box and a moderator and target were added to the setup. The top counter was added at the end of the second week, and at the same time, the telescope was connected to the computer. The neutron detector completed the set-up at the beginning of the third week.

I was unable to complete the experiment before this project ended; often in studies of cosmic radiation, such as this one, results cannot be expected immediately. Despite this, however, I think the time spent becoming familiar with the background of the experiment, acquiring knowledge of the principles of operation of scintillation detectors and counting electronics, assembling the detectors and the telescope, and then just searching for $\mu^{-}$has been more than worthwhile. 


\section{ACKNOWLEDGEMENTS}

The disproportionate amount of attention given thus far to the scientific aspects of this project unfairly neglects to recognize the individuals who have played such major roles in making this project as worthwhile as it has been.

I would like to thank the Senior Study Project Committee at Allendale Columbia for giving students opportunities to design their own learning experiences;

all the scientists at NSRL, for unknowingly inspiring me by their long hours and hard work;

Prof. Schroeder, for organizing my project and allowing me to work with him;

Shawn Baldwin, a graduate student at NSRL, for the superhuman patience he has shown; the progress I have made would not have been possible without his help;

and Dr. Mandeville, my humorous and perceptive physics teacher, who is principally responsible for my interest in physics.

This work was supported by the U.S. Department of Energy under Grant No. DE-FG0288ER40414(University of Rochester). 


\section{REFERENCES}

[1] F. Close, M. Marten, and C. Sutton, The Particle Explosion, Oxford University Press (New York, 1987), 67-8.

[2] W. R. Leo, Techniques for Nuclear and Particle Physics Experiments, Springer-Verlag (New York, 1987), 149-205. 


\section{FIGURES}

FIG. 1. An illustration of $\mu^{-}$capture by a target atom. In picture (a), a $\mu^{-}$, which acts like an electron, replaces an electron in the outermost orbits of a target (lead) atom. In picture (b), muonic X-rays are emitted as the muon loses energy and drops to a lower atomic orbital. Picture (c) and (d) are close-up views of the nucleus. In (c), the $\mu^{-}$reached a low enough orbital to be captured by the lead nucleus. In (d), it interacts with protons in the nucleus to form neutrons and neutrinos $\left(\mu^{-}+p \rightarrow \nu_{\mu}+n\right) \cdot \gamma$-rays and neutrons are emitted from nucleus capture.

FIG. 2. Two views of a plastic scintillation detector. The scintillator is a 6 " $\times 6 " x .25$ " piece of plastic. Light from the thin edge of the scintillator is transmitted to the round photomultiplier tube via a light guide, which has a round 'foot' to accommodate the round surface the photomultiplier. Together, the scintillator and light guide form a 'paddle' which is coupled to the photomultiplier tube by grease. A mu metal shield is placed around the tube to prevent magnetic fields from bending the paths of electrons in it.

FIG. 3. A photomultiplier tube [2]. Voltage is first applied to the photomultiplier base. When a photon from the scintillator reaches the cathode (the negative electrode) in the photomultiplier, an electron is emitted, but the applied voltage causes it to accelerate towards the first dynode (one component of the electron collection system in the tube). When it strikes the dynode, it causes other electrons to be emitted and they accelerate towards the next dynode. The process repeats itself until the the electrons reach the anode (the positively charged electrode), where they are all collected to produce a current that can be amplified and analyzed.

FIG. 4. Experimental set-up of the muon telescope. The three scintillation detectors are labelled $\mathrm{C} 1-\mathrm{C} 3$, the moderator is $\mathrm{M}$, the target, $\mathrm{T}$, and the neutron detector, $\mathrm{C} 4$. PM stands for photomultiplier. 
FIG. 5. A plot which compares the fraction of particles stopped with various moderator thicknesses for different targets. The thickest targets stopped the greatest fractions of particles, but changing the moderator thicknesses in these targets did not affect these fractions as much as it did in the two inch target. With a two inch target, the six inch thick target stopped the greatest percentage of particles $(69 \%)$.

FIG. 6. A diagram of some electronics involved. High voltage (HV) is applied to the detectors. The anode signals from the counters pass through discriminators and lead to a logic module. C1 in position $A$ is connected to detect coincidence with $\mathrm{C} 2$ in position $\mathrm{B}$. The signal from $\mathrm{C} 3$ in the veto position completes the requirement that particles stop in the target. A signal from the logic module forms a start signal for the TAC. The neutron detector generates the stop signal. The TAC is connected to the MCA (computer).

FIG. 7. Examples of analog and logic time pulses, sketched from an oscilloscope. The amplitudes of analog signals (a) depend on the energies of the pulses. Logic time pulses (b) are simply 'yes' or 'no' signals for pulses.

FIG. 8. A more simplified diagram of the electronics involved. After a proper signal has been generated (1) and processed (2), it must be digitized before the computer can use it. The hardware interface (3) are the modules (such as the ADC) that do this. The same signal (4) is also used to trigger the computer. When the computer is prepared to receive data, it sends a signal (5) back to the digitizers to transmit the now-prepared signal (6).

FIG. 9. An example of current output from the MCA. The $x$-axis displays the channel numbers of the MCA, which are similar to pixels on a computer screen. Each channel represents a delay time. The y-axis displays the number of counts, or yield, for that delay time. The output displayed here is consistent with the presence of $\mu^{-}$in the target: the 'prompt' peak (representing muonic $\mathrm{X}$-rays), followed by an exponentially decreasing decay curve (representing the decay of the muons) was expected. Further measurements will confirm this. 


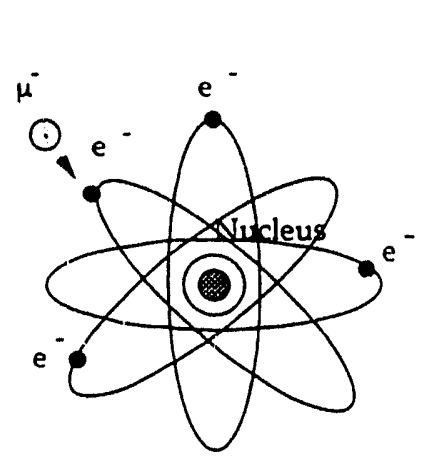

(a)

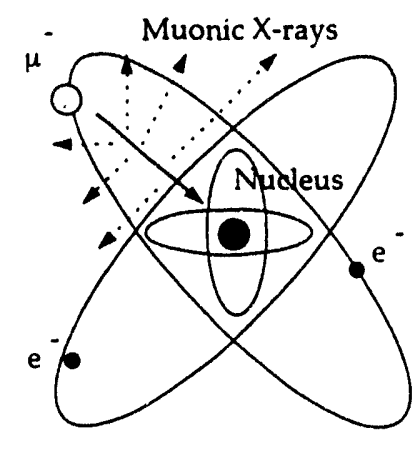

(b)

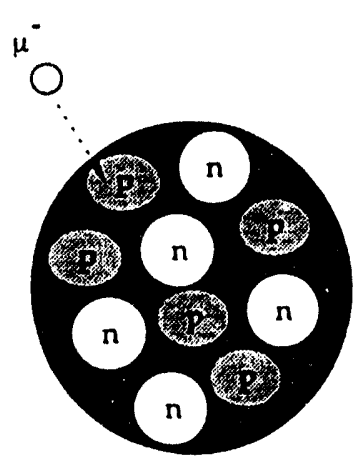

(c)

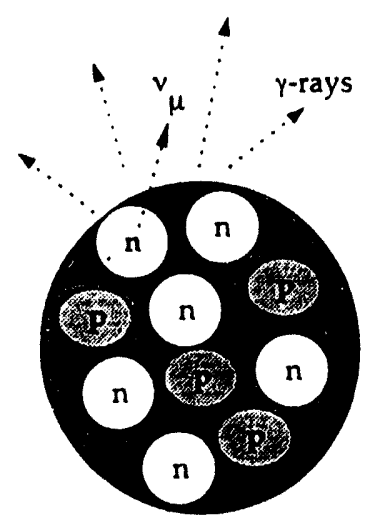

(d)

Figure 1. 


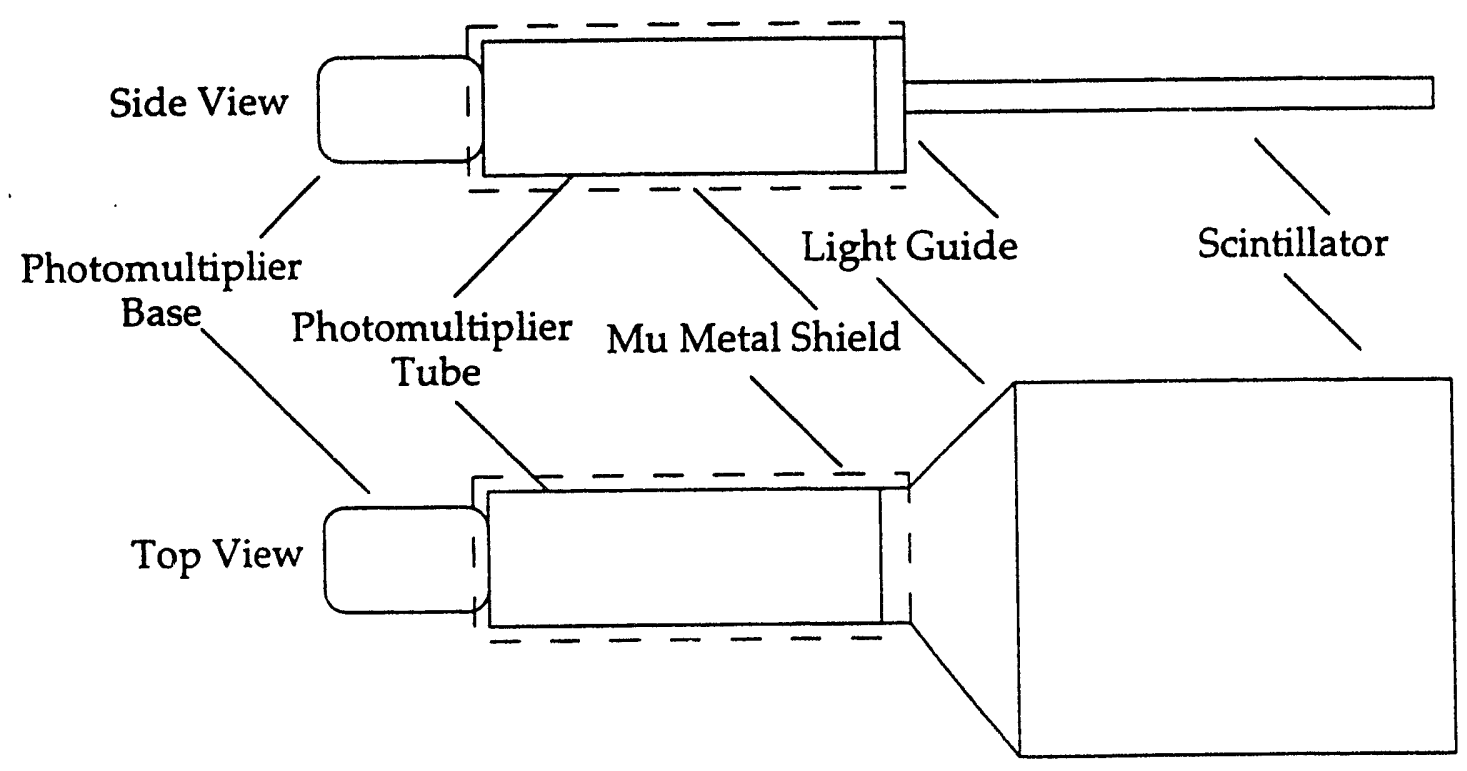

Figure 2.

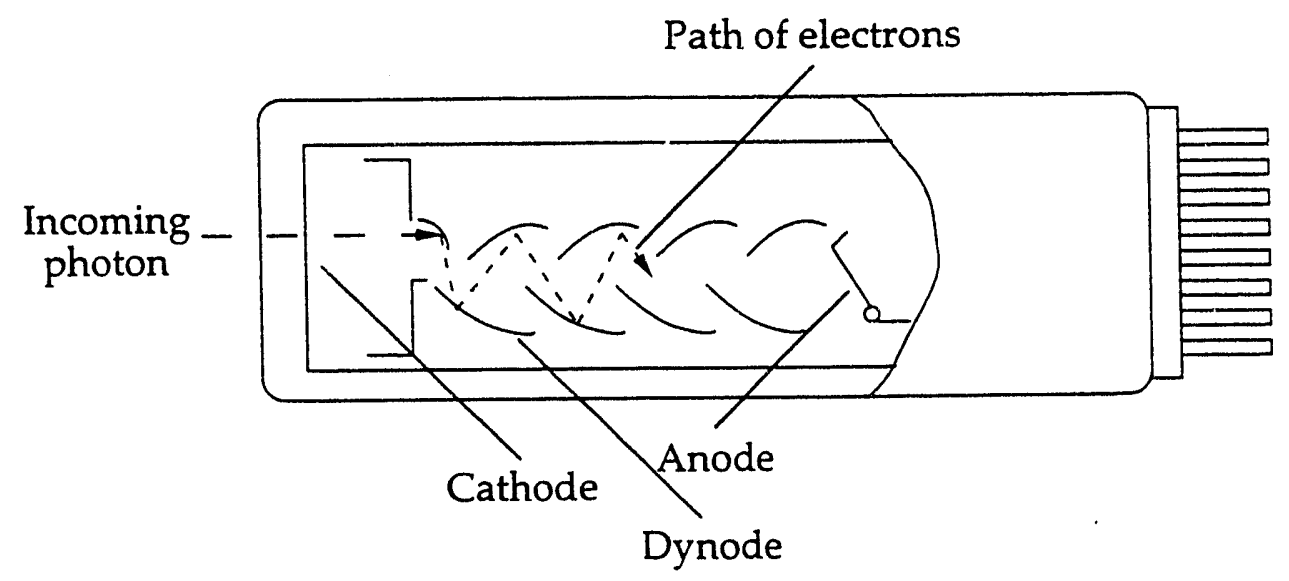

Figure 3. 


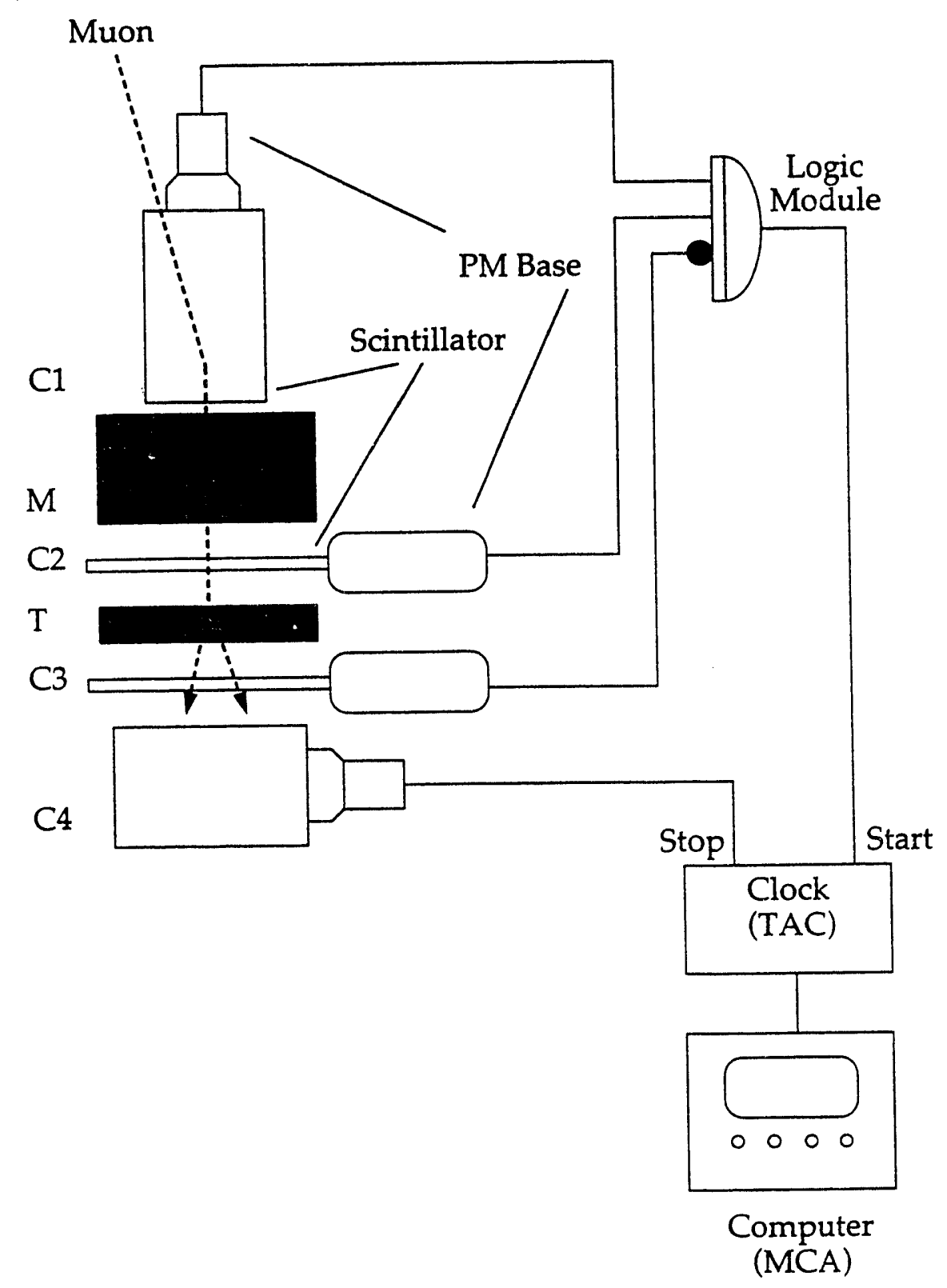

Figure 4 . 
Varying mod. thickness with different targets

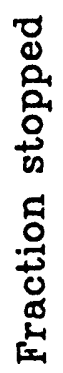

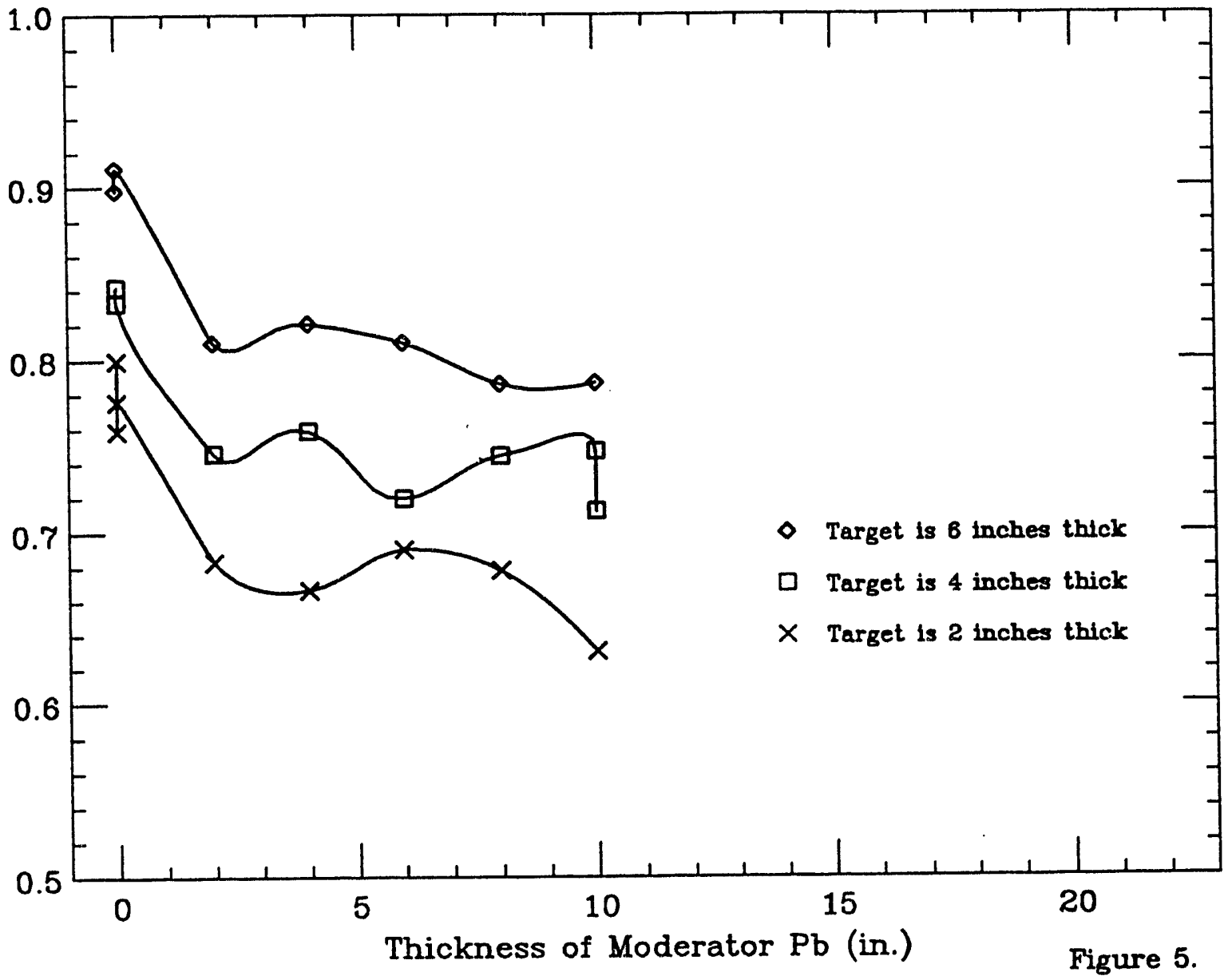




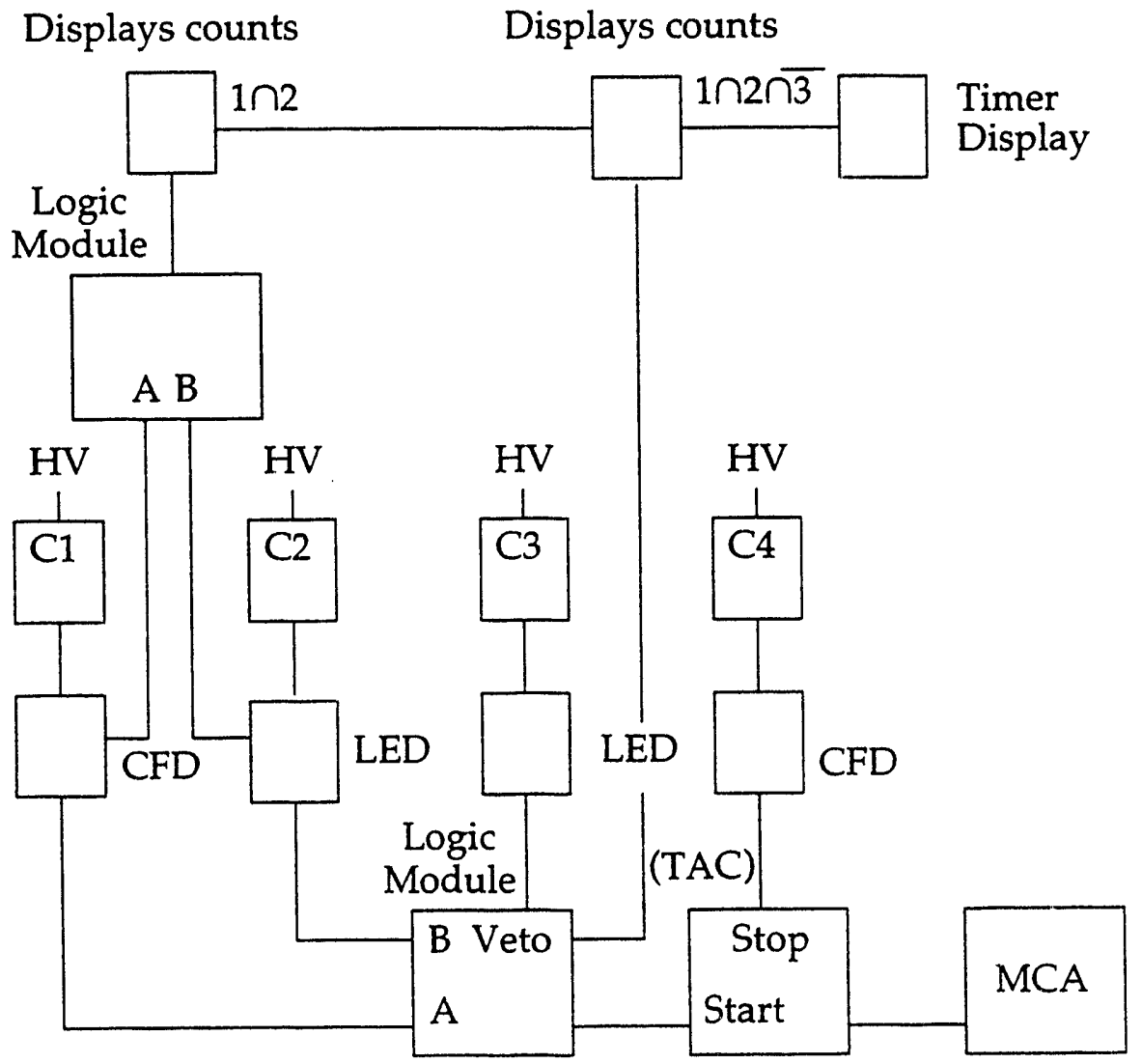

Figure 6. 
Oscilloscope Display

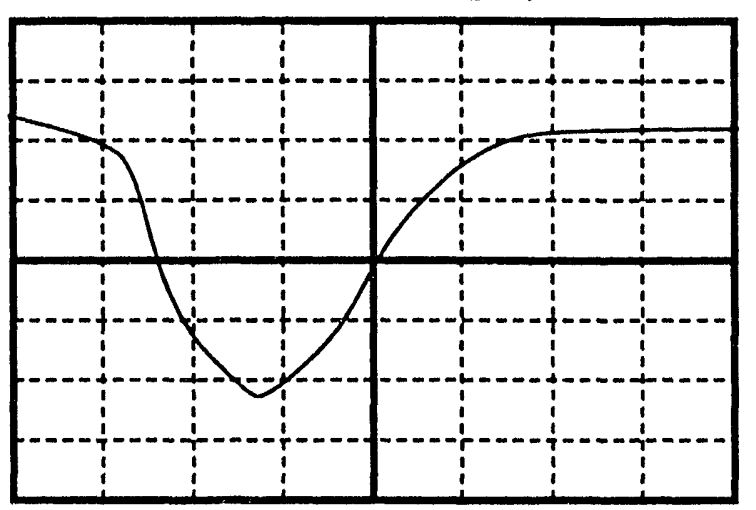

(a)
Oscilloscope Display

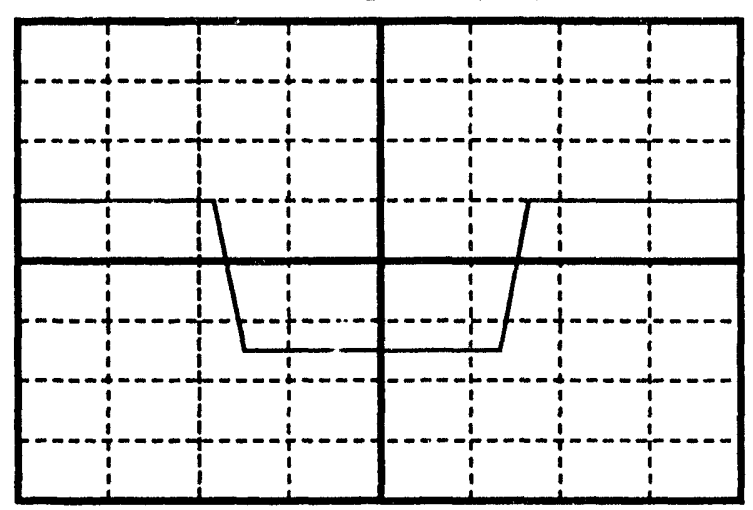

(b)

Figure 7.

4.

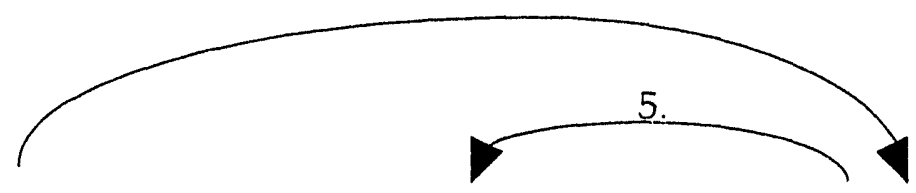

1. Signal Generation 2. Signal Processing 3. Hardware Interface Data Acquisition Detectors Electronics

Figure 8. 
6 วมกริเม

Number of Counts or Yield

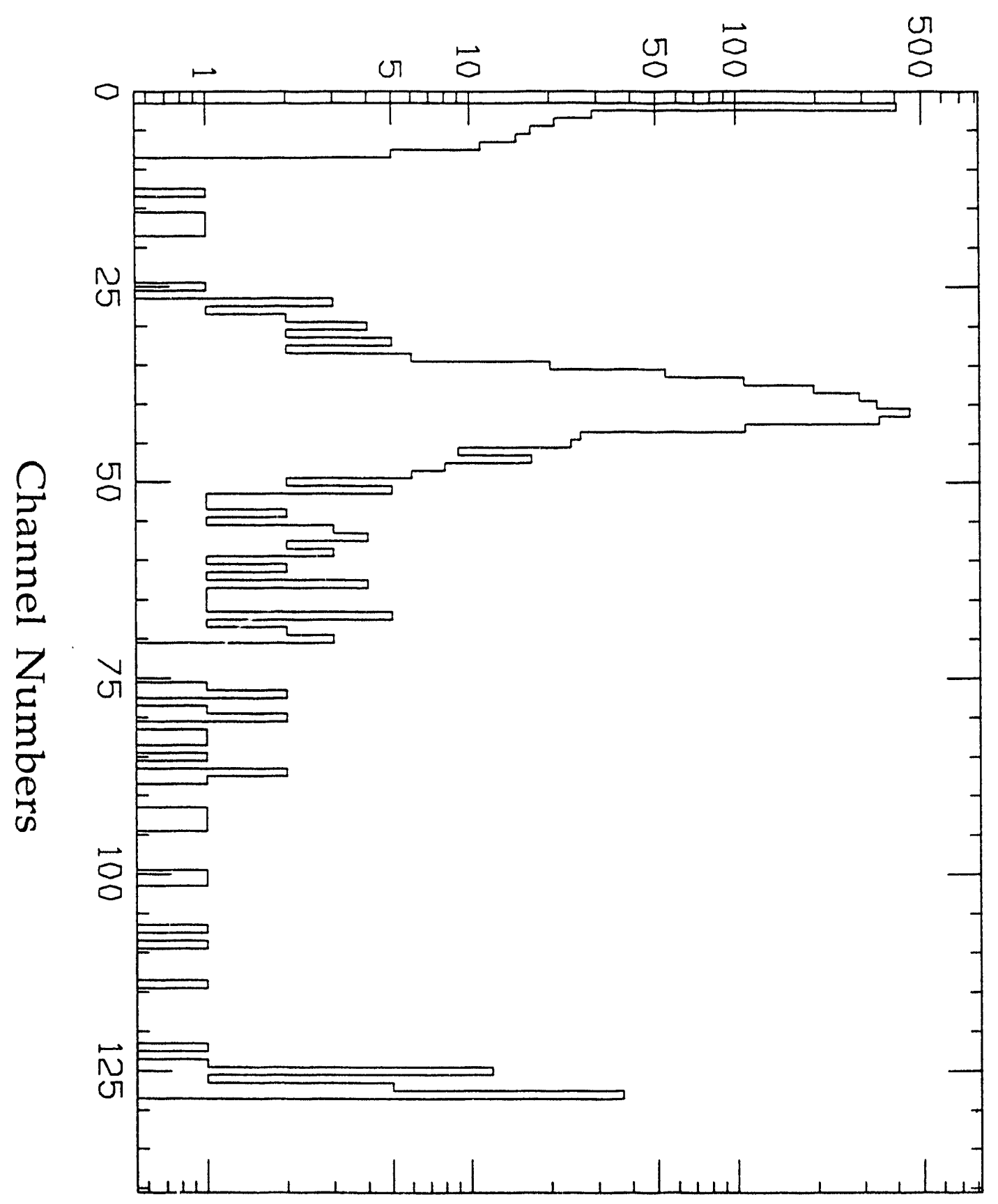


J.L. Wile, S.S. Datta, W.U. Schröder, J. Tõke, D. Pade, S.P. Baldwin, J.R.

Huizenga, B.M. Quednau, R.T. deSouza, and B.M. Szabo

Thermal Properties of Compound Systems Formed in Fusion of ${ }^{28} \mathrm{Si}$ Ions with $118 \mathrm{Sn}$ and ${ }^{124} \mathrm{Sn}$ Nuclei

Physical Review C 47, 2135 (1993).

J.R. Huizenga,

"Are the World's Energy Problems Over?",

Leaders Magazine 16, no. 1, page 21 (1993).

M.B. Chatterjee, S.P. Baldwin, J.R. Huizenga, D. Pade, B.M. Quednau, W.U. Schröder, B.M. Szabo and J. Tõke,

"Reply to 'Energy Partition in Near-Barrier Strongly Damped Collisions $58 \mathrm{Ni}+{ }^{208} \mathrm{~Pb}^{\prime \prime}$,

Phys. Rev. C47, 3003 (1993).

B.M. Quednau, S.P. Baldwin, M.B. Chatterjee, J.R. Huizenga, W.U. Schröder, B.M. Szabo, J. Tõke, U. Jahnke, D. Hilscher, H. Rossner, B. Lott, S. Bresson, J. Galin, D. Guerreau, M. Morjean, and D. Jacquet, "Binary Collision Dynamics in the Reaction ${ }^{197} \mathrm{Au}+{ }^{208} \mathrm{~Pb}$ at $29 \mathrm{MeV} / \mathrm{Nucleon}$ ", Physics Letters B 309, 10 (1993), in press.

J. Tõke, S.P. Baldwin, B. Lott, S.A. Masserant, and W.U. Schröder, "A Simple Pulse Processing Concept for a Low-Cost Pulse-Shape-Based Particle Identification"

Nuclear Instruments and Methods, (1993 ), in press.

W.U. Schröder, "Unexpected Features of Heavy-Ion Reactions at Intermediate Energies", Proceedings of the International School-Seminar on Heavy-Ion Physics, Dubna, 1993, (in press). 


\section{IV.C. Invited Lectures}

"Cold Fusion: The Scientific Fiasco of the Century"

Colloquium, Laboratory for Laser Energetics, University of Rochester, September 11, 1992, by J.R. Huizenga

"Cold Fusion: The Scientific Fiasco of the Century"

Meeting of Society of Health Physicists in Syracuse, New York

September 18, 1992, by J.R. Huizenga

"Cold Fusion: An Evaluation of the Claimed Evidence"

Laboratory Colloquium, Los Alamos National Laboratory, Los Alamos, New Mexico,

September 22, 1992, by J.R. Huizenga

"The Cold Fusion Fiasco"

Colloquium, Tokyo Metropolitan University, Tokyo, Japan,

October 20, 1992, by J.R. Huizenga

"Cold Fusion: The Scientific Fiasco of the Century"

Buffalo Section of the American Chemical Society, Buffalo, New York

November 17, 1992, by J.R. Huizenga

"Cold Fusion: The Scientific Fiasco of the Century"

Calvin College, Grand Rapids, Michigan,

November 19, 1992, by J.R. Huizenga

"Cold Fusion: The Scientific Fiasco of the Century"

Hope College, Holland, Michigan,

November 20, 1992, by J.R. Huizenga

"Binary Character of Collisions Between Very Heavy Nuclei Around $30 \mathrm{MeV} /$

Nucleon"

Seminar, DAPNIA, Ormes les Merisiers (France),

November 20, 1992, by B. Lott 
"Binary Character of Collisions Between Very Heavy Nuclei Around $30 \mathrm{MeV} /$

Nucleon",

Seminar, GANIL, Caen (France),

November 27, 1992, by B. Lott

"The Dissipative Reaction ${ }^{197} \mathrm{Au}+208 \mathrm{~Pb}$ at $\mathrm{E}_{\mathrm{lab}} / \mathrm{A}=29 \mathrm{MeV}^{\prime \prime}$

Seminar, Nuclear Structure Research Laboratory, University of Rochester,

December 10, 1992, by B.M. Quednau

"Cold Fusion Fiasco"

Colloquium, Princeton Plasma Physics Laboratory, Princeton University,

December 16, 1992, by J.R. Huizenga

"The Dynamics of Collisions Between Very Heavy Ions",

Invited Seminar, Niels Bohr Institute, Copenhagen (Denmark),

February 8, 1993, by W. U. Schröder

"The Cold Fusion Fiasco"

Retired Chemists Luncheon Meeting, Rochester American Chemical Society,

Rochester, NY, February 24, 1993, by J.R. Huizenga

"Nuclear Rainbow at $4 \mathrm{GeV}$ ?"

Seminar, Nuclear Structure Research Laboratory, University of Rochester, April 1, 1993, by S. P. Baldwin

"The Cold Fusion Fiasco"

Seminar, Department of Chemistry, University of Virginia,

April 28, 1993, by J.R. Huizenga

"Unexpected Features of Heavy-Ion Reactions at Intermediate Energies"

Invited talk, International School-Seminar on Heavy-Ion Physics, Dubna

(Russia), May 10-15, 1993, by W. U. Schröder

"Collective Dynamics of Interactions between Very Heavy Ions at Fermi

Energies", 
Seminar, Kurchatov Institute of Atomic Energy, Moscow (Russia), May 17, 1993, by W. U. Schröder

"Collective Dynamics of Interactions between Very Heavy Ions at Fermi Energies",

Seminar, Khlopin Radium Institute, St. Petersburg (Russia), May 19, 1993, by W. U. Schröder

"Collective Dynamics of Intermediate-Energy Heavy-Ion Collisions", Invited Talk, Symp. "Hot Nucl. Matter", Meeting of the American Chemical Society, Chicago, August 24, 1993, by W. U. Schröder

\section{IV.D. Contributed Papers at Professional Meetings}

B. Lott, S.P. Baldwin, B.M. Szabo, B.M. Quednau, W.U. Schröder, J. Tõke, L.G. Sobotka, J. Barreto, R.J. Charity, L. Gallamore, D.G. Sarantites, D.W. Stracener, R.T. de Souza, "Non-Evaporative Emission of Intermediate-Mass Fragments in Peripheral ${ }^{209} \mathrm{Bi}+{ }^{136} \mathrm{Xe}$ Collisions at $\mathrm{E}_{\mathrm{Lab}} / \mathrm{A}=28.2 \mathrm{MeV}$, presented by $\mathrm{B}$. Lott at the APS Meeting, Washington, D.C., April 1993, Bulletin of the American Physical Society 38, 941 (1993)

S.P. Baldwin, B. Lott, B.M. Szabo, B.M. Quednau, W.U. Schröder, J. Tõke, L.G. Sobotka, J. Barreto, R.J. Charity, L. Gallamore, D.G. Sarantites, D.W. Stracener, R.T. de Souza, "Correlations Between Massive Fragments and Light Particles in the Reaction ${ }^{209} \mathrm{Bi}+{ }^{136} \mathrm{Xe}$ Collisions at ELab/A $=28.2 \mathrm{MeV}$ ", presented by S.P. Baldwin at the APS Meeting, Washington, D.C., April 1993, Bulletin of the American Physical Society 38, 941 (1993)

X. Zhao, S.P. Baldwin, W. U. Schröder, J. Tõke, "Development of a $4 \pi$ Neutron Detector for Iniermediate Energy Heavy-Ion Reactions", presented by X. Zhao at the APS Meeting, Washington, D.C., April 1993, Bulletin of the American Physical Society 38, 998 (1993)

S.A. Masserant, J. Tõke, S.P. Baldwin, B. Lott, B.M. Quednau, W.U. Schröder, X. Zhao, "A Simple, Highly Economical Particle Identification 
Method Based on Pulse Shape Analysis", presented by S.P. Baldwin at the APS Meeting, Washington, D.C., April 1993,

Bulletin of the American Physical Society 38, 1036 (1993)

\section{IV.E. Presentations before NSF and NSAC Committees}

"Kinetics of Nuclear Fusion and Thermodynamics of Composite Nuclei", Presentation before the NSF Site Visiting Committee, Nuclear Structure Research Laboratory, Rochester, New York

September 10, 1992, by W. U. Schröder

"Design and Applications of the SuperBall Neutron Calorimeter", Presentation before the NSAC Subcommittee, NSF Site Visit at the National Superconducting Cyclotron Laboratory, East Lansing, Michigan December 8, 1992, by W. U. Schröder

"Formation and Decay of Composite Nuclei at High Excitation Energies", Presentation before the NSAC Subcommittee, NSF Site Visit at the Nuclear Structure Research Laboiatory, Rochester, New York February 3, 1993, by W. U. Schröder

"Investigations of Intermediate-Energy Heavy-Ion Reactions by the Rochester Nuclear Chemistry Group", Public Comment before NSAC, Washington Meeting, Washington, D.C., February 26, 1993, by W. U. Schröder

\section{IV.F. Ph.D. and M.S. Degrees}

B. M. Quednau

"Dissipative Reaction Dynamics of ${ }^{197} \mathrm{Au}+{ }^{208} \mathrm{~Pb}$ Collisions at a Bombarding Energy of $E / A=29 \mathrm{MeV} "$

Ph.D. Thesis, submitted December 1992

Sharon A. Masserant

Master of Science, May 1993 


\section{IV.G. Professional Activities}

Member of the American Physical Society, Division of Nuclear Physics

Member of the American Chemical Society, Division of Nuclear Chemistry and Technology

Member of ATLAS Program Advisory Committee

Member of Users' Executive Committee, National Superconducting Cyclotron

Laboratory, Michigan State University

Referee for Journal Articles and Proposals to Funding Agencies

Member of University Computing VAX-VMS Committee, University of Rochester

Member or Chair of several ad hoc and search committees, University of Rochester

Chairman and/or member of Ph.D. examining committees, University of Rochester Member and Chair of Services and Space Committee, University of Rochester, Department of Chemistry Member of Computing Committee, University of Rochester, Department of Chemistry Member of Nuclear Structure Research Laboratory Steering Committee, University of Rochester Member of Nuclear Structure Research Laboratory Computing Committee, University of Rochester 


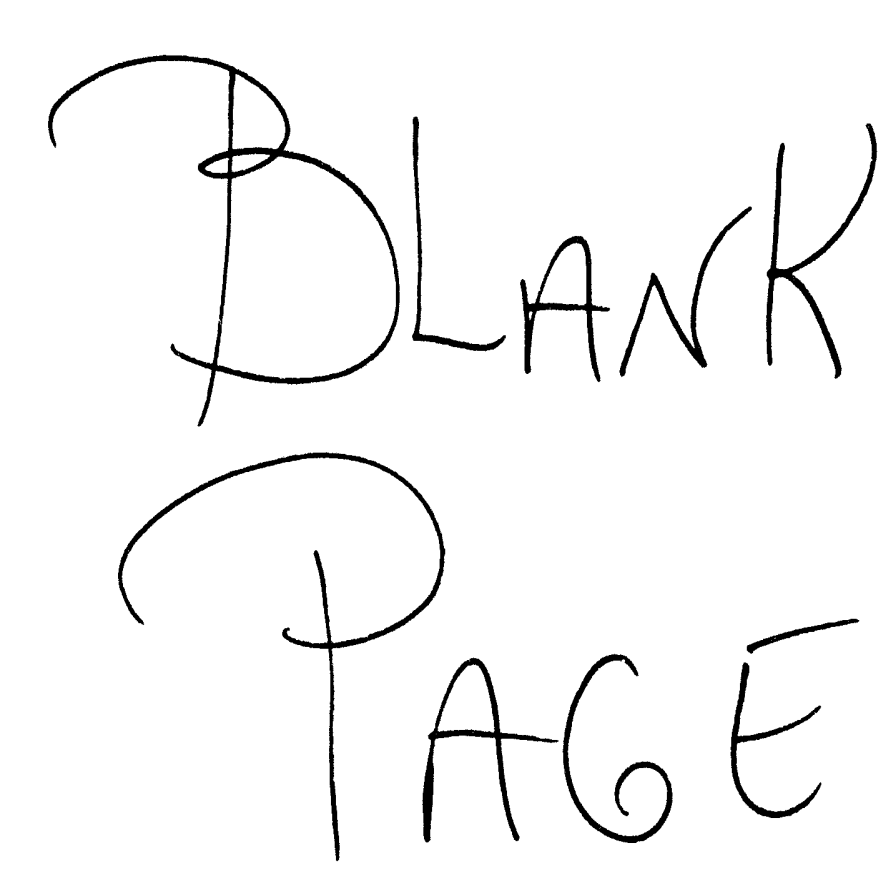




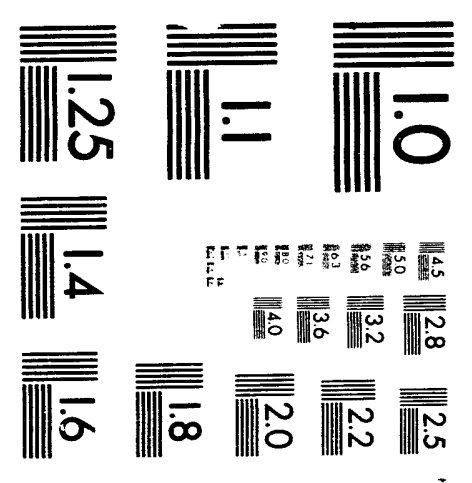



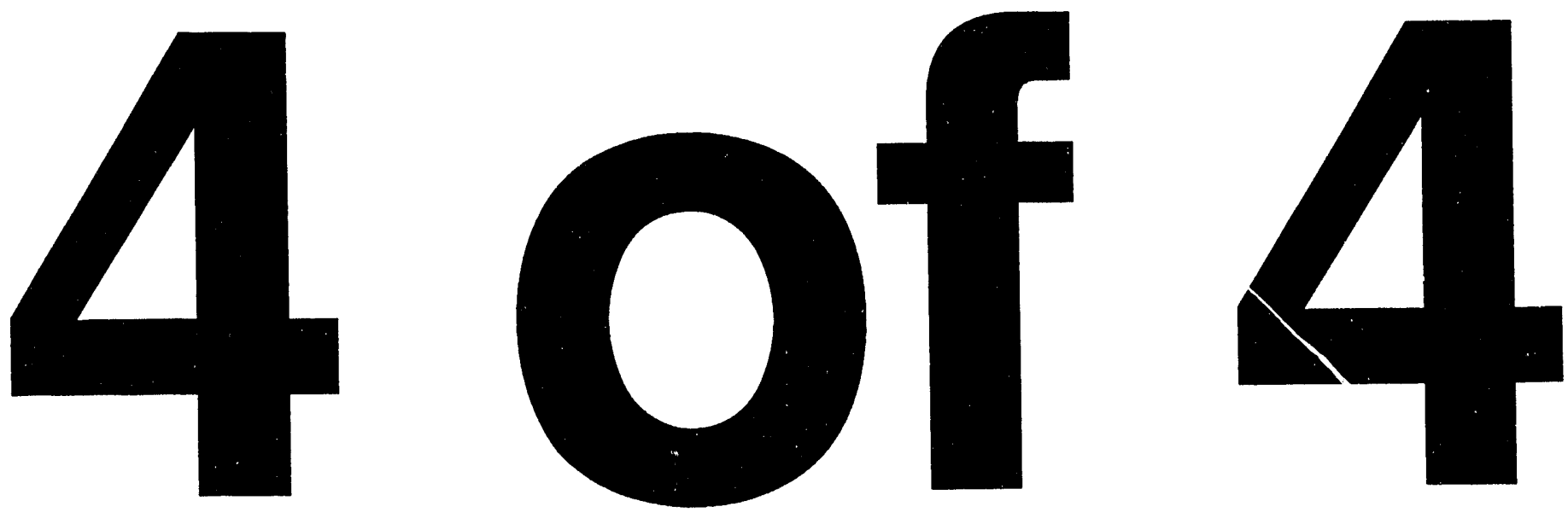


\section{Personnel}

Mr. S. P. Baldwin, Graduate Student, Department of Chemistry

Dr. I.M. Govil, Visiting Scientist ${ }^{1}$

Dr. J.R. Huizenga, Tracy H. Harris Professor Emeritus of Chemistry and Physics, Senior Faculty Consultant

Ms. K. $\mathrm{Ho}^{2}$

Ms. E. Katta 3

Dr. B. Lott, Research Associate 4

Ms. S. A. Masserant, Graduate Student, Department of Chemistry 5

Mr. B.M. Quednau, Graduate Student, Department of Chemistry 6

Dr. W.U. Schröder, Professor of Chemistry

Dr. J. Tõke, Senior Research Associate

Mr. Xiaofei Zhao, Graduate Student, Department of Physics

${ }^{1}$ During May/June 1993

2Senior High School Project, April/May 1993

${ }^{3}$ ACS SEEDS High School Fellow, June-August 1993

${ }^{4}$ On Leave of Absence from CRN Strasbourg, France, until April 1993

5Until June 1993

6Until December 1993

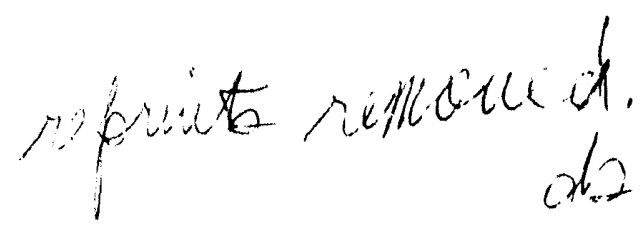



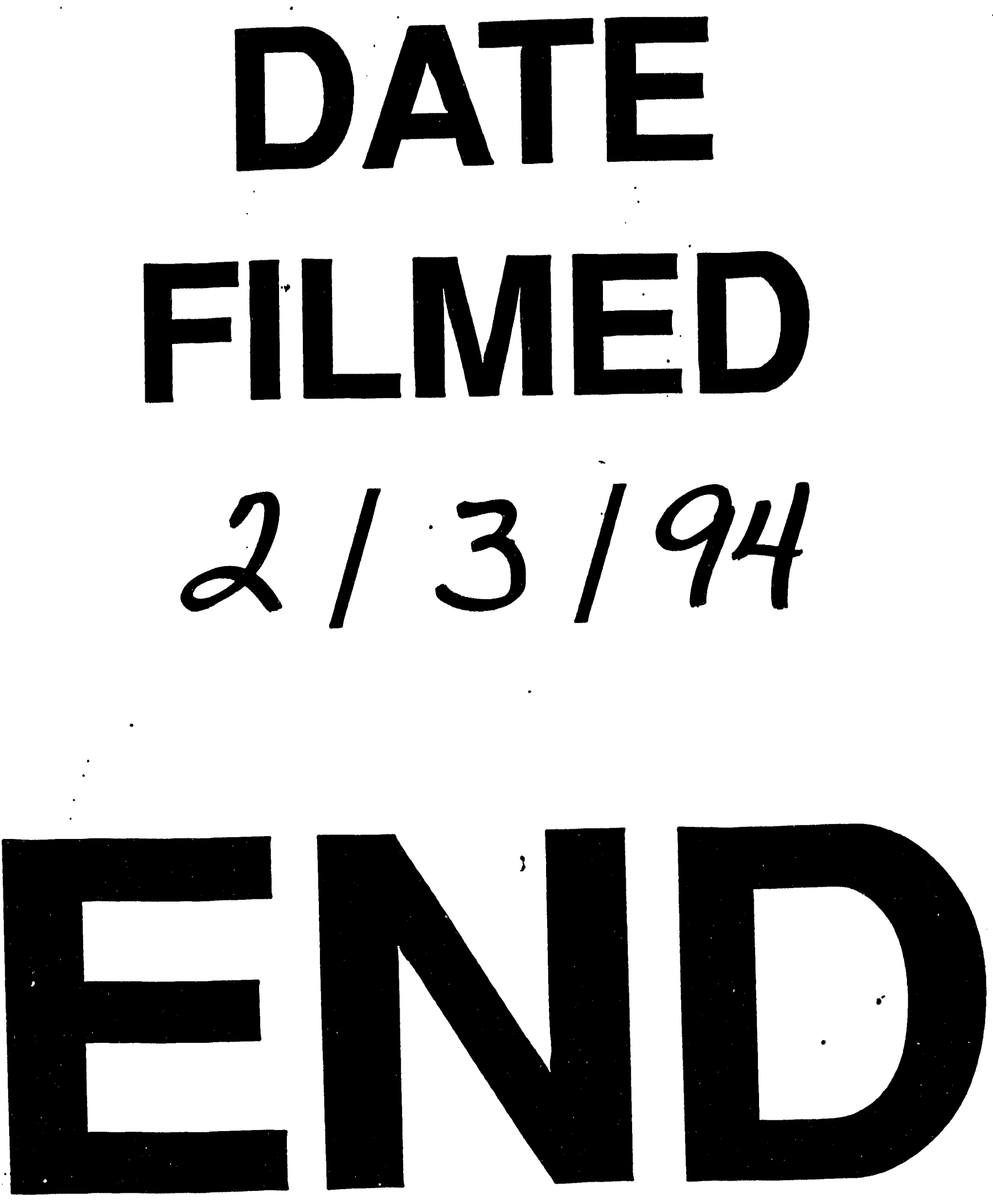
